$\operatorname{Argonne} \underset{\bigotimes}{\bigotimes}$

\title{
Annual Report of Groundwater Monitoring at Centralia, Kansas, in 2010
}

\section{Environmental Science Division}


About Argonne National Laboratory

Argonne is a U.S. Department of Energy laboratory managed by UChicago Argonne, LLC under contract DE-AC02-06CH11357. The Laboratory's main facility is outside Chicago, at 9700 South Cass Avenue, Argonne, Illinois 60439. For information about Argonne and its pioneering science and technology programs, see www.anl.gov.

\section{Availability of This Report}

This report is available, at no cost, at http://www.osti.gov/bridge. It is also available on paper to the U.S. Department of Energy and its contractors, for a processing fee, from:

U.S. Department of Energy

Office of Scientific and Technical Information

P.O. Box 62

Oak Ridge, TN 37831-0062

phone (865) 576-8401

fax (865) 576-5728

reports@adonis.osti.gov

\section{Disclaimer}

This report was prepared as an account of work sponsored by an agency of the United States Government. Reference herein to any specific commercial product, process, or service by trade name, trademark, manufacturer, or otherwise, does not necessarily constitute or imply its endorsement, recommendation, or favoring by the United States Government or any agency thereof. The views and opinions of document authors expressed herein do not necessarily state or reflect those of the United States Government or any agency thereof, Argonne National Laboratory, or UChicago Argonne, LLC. 


\section{Annual Report of Groundwater Monitoring at Centralia, Kansas, in 2010}

by

Applied Geosciences and Environmental Management Section

Environmental Science Division, Argonne National Laboratory

March 2011

USDA 


\section{Contents}

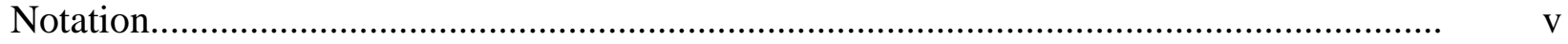

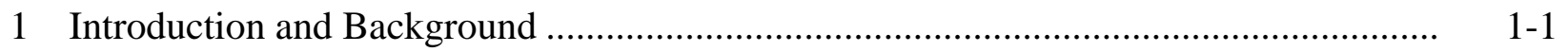

2 Sampling and Analysis Activities........................................................................

2.1 Measurement of Groundwater Levels............................................................... 2 2-1

2.2 Monitoring Well and Piezometer Sampling and Analyses ................................... 2 2-1

2.3 Handling and Disposal of Investigation-Derived Waste ..................................... 2 2-2

2.4 Quality Control for Sample Collection, Handling, and Analysis ........................ . 2-2

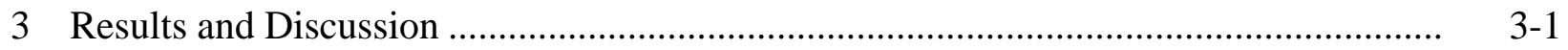

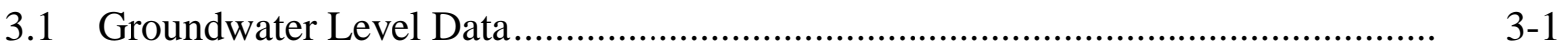

3.2 Groundwater Analysis Results................................................................... 3-2

3.2.1 Sitewide Monitoring Results............................................................... 3-2

3.2.2 Monitoring Results for the IM Pilot Test Area ...................................... 3-3

4 Conclusions and Recommendations …...............................................................

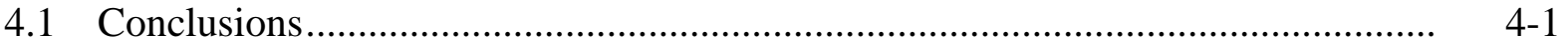

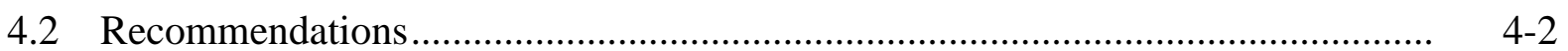

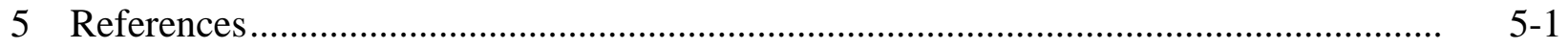

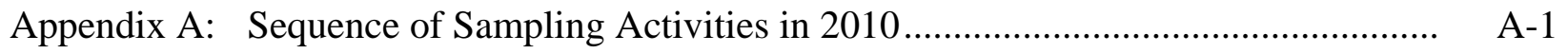

Appendix B: Quality Control Data Summary …................................................... B-1

Appendix C: Time Series Diagrams for Selected Parameters at IM Monitoring Points..... C-1

Supplement 1: Waste Characterization and Disposal Documentation............................. on CD

Supplement 2: Data Summaries for Verification VOCs Analyses by TestAmerica Laboratories, Inc. .............................................................................. on CD

\section{Tables}

3.1 Hand-measured water levels at Centralia in 2010 ............................................ 3-6 
3.2 Analytical results from the AGEM laboratory for volatile organic compounds in groundwater samples collected from the sitewide monitoring points at Centralia, August 2004 to September 2010

3.3 Field measurements for groundwater samples collected from the sitewide monitoring points at Centralia, August 2004 to September 2010.

3.4 Analytical results from the AGEM laboratory for volatile organic compounds in groundwater samples collected from the IM pilot test monitoring points at Centralia, September 2008 to September 2010

3.5 Field measurements for groundwater samples collected from the IM pilot test monitoring points at Centralia, September 2008 to September 2010

A.1 Sequence of sampling activities at Centralia in 2010

B.1 Analytical results from the AGEM Laboratory for quality control samples collected in 2010

B.2 Analytical results for verification groundwater samples analyzed at the AGEM Laboratory and by TestAmerica

\section{Figures}

1.1 Currently approved annual sitewide monitoring network at Centralia

1.2 Pilot test monitoring points currently approved for annual or twice-yearly sampling.....

3.1 Hydrographs summarizing results of long-term water level monitoring at Centralia, January 2009 to September 2010

3.2 Potentiometric surface at Centralia, based on water levels measured manually on April 28, 2010

3.3 Carbon tetrachloride concentrations in groundwater in the sitewide monitoring wells sampled in September 2010, with the interpreted lateral extent of the contaminant at intervals during the period August 2004 to September 2010

3.4 Carbon tetrachloride in groundwater samples collected during the pre-injection baseline sampling, September and November 2007

3.5 Field-measured results for DO in groundwater samples collected during the pre-injection baseline sampling, September and November 2007. 
3.6 Field-measured results for ORP in groundwater samples collected during the pre-injection baseline sampling, September and November 2007.

3.7 Analytical results for carbon tetrachloride in groundwater samples collected in September 2010 and October 2009 at the IM pilot test monitoring points.

3.8 Field-measured results for DO in groundwater samples collected in September 2010 and October 2009 at the IM pilot test monitoring points

3.9 Field-measured results for ORP in groundwater samples collected in September 2010 and October 2009 at the IM pilot test monitoring points

C.1 Analytical results for VOCs, DO, and ORP in groundwater samples collected at location MW02, November 2007 to September 2010

C.2 Analytical results for VOCs, DO, and ORP in groundwater samples collected at location PMP1, January 2008 to September 2010

C.3 Analytical results for VOCs, DO, and ORP in groundwater samples collected at locations PSB2 and PMP2, November 2007 to September 2010

C.4 Analytical results for VOCs, DO, and ORP in groundwater samples collected at locations PSB3 and PMP3, November 2007 to September 2010

C.5 Analytical results for VOCs, DO, and ORP in groundwater samples collected at location PMP4, January 2008 to September 2010

C.6 Analytical results for VOCs, DO, and ORP in groundwater samples collected at locations PSB5 and PMP5, November 2007 to September 2010

C.7 Analytical results for VOCs, DO, and ORP in groundwater samples collected at locations PSB6 and PMP6, November 2007 to September 2010

C.8 Analytical results for VOCs, DO, and ORP in groundwater samples collected at locations PSB7 and PMP7, November 2007 to September 2010

C.9 Analytical results for VOCs, DO, and ORP in groundwater samples collected at locations PSB8 and PMP8, November 2007 to September 2010

C.10 Analytical results for VOCs, DO, and ORP in groundwater samples collected at locations PSB9 and PMP9, November 2007 to September 2010 


\section{Notation}

AGEM Applied Geosciences and Environmental Management

AMSL above mean sea level

BGL below ground level

${ }^{\circ} \mathrm{C} \quad$ degree(s) Celsius

CAS Corrective Action Study

CCC Commodity Credit Corporation

CD compact disc

COC chain of custody

DO dissolved oxygen

EPA U.S. Environmental Protection Agency

$\mathrm{ft} \quad$ foot (feet)

IM interim measure

in. inch(es)

ISCR in situ chemical reduction

KDHE Kansas Department of Health and Environment

L liter(s)

$\mu \mathrm{g} / \mathrm{L} \quad$ microgram(s) per liter

$\mu \mathrm{S} / \mathrm{cm} \quad$ microsiemen(s) per centimeter

$\mathrm{mg} / \mathrm{L} \quad$ milligram(s) per liter

mi mile(s)

$\mathrm{mV} \quad$ millivolt(s)

ORP oxidation-reduction potential

RBSL risk-based screening level

TOC top of casing

USDA U.S. Department of Agriculture

VOC volatile organic compound

yr year(s) 


\section{Annual Report of Groundwater Monitoring at Centralia, Kansas, in 2010}

\section{Introduction and Background}

In September 2005, periodic sampling of groundwater was initiated by the Commodity Credit Corporation of the U.S. Department of Agriculture (CCC/USDA) in the vicinity of a grain storage facility formerly operated by the CCC/USDA at Centralia, Kansas. The sampling at Centralia is performed on behalf of the CCC/USDA by Argonne National Laboratory, in accord with a monitoring program approved by the Kansas Department of Health and Environment (KDHE). The objective is to monitor levels of carbon tetrachloride contamination identified in the groundwater at Centralia (Argonne 2003, 2004, 2005a).

Under the KDHE-approved monitoring plan (Argonne 2005b), the groundwater was sampled twice yearly from September 2005 until September 2007 for analyses for volatile organic compounds (VOCs), as well as measurement of selected geochemical parameters to aid in the evaluation of possible natural contaminant degradation processes (reductive dechlorination) in the subsurface environment (Argonne 2006, 2007a, 2008a). The results from the two-year sampling program demonstrated the presence of carbon tetrachloride contamination at levels exceeding the KDHE Tier 2 risk-based screening level (RBSL) of $5 \mu \mathrm{g} / \mathrm{L}$ for this compound, in a localized groundwater plume that has shown little movement. The relative concentrations of chloroform, the primary degradation product of carbon tetrachloride, suggested that some degree of reductive dechlorination or natural biodegradation was talking place in situ at the former CCC/USDA facility on a localized scale.

The CCC/USDA subsequently developed an Interim Measure Conceptual Design (Argonne 2007b), proposing a pilot test of the Adventus EHC technology for in situ chemical reduction (ISCR). The proposed interim measure (IM) was approved by the KDHE in November 2007 (KDHE 2007). Implementation of the pilot test occurred in November-December 2007. The objective was to create highly reducing conditions that would enhance both chemical and biological reductive dechlorination in the injection test area (Argonne 2009a).

The KDHE (2008a) requested that sitewide monitoring continue until a final remedy is selected (as part of a Corrective Action Study [CAS] evaluation) and implemented. In response to this request, the established sampling across the site and additional sampling in the IM pilot test area continued in 2008 (Argonne 2008b, 2009a,b). 
On the basis of results of the 2005-2008 sitewide monitoring and the 2008 IM pilot test monitoring, the CCC/USDA recommended a revised sampling program for both the wider site and the IM pilot test area (Section 4.2 in Argonne 2009b). The elements of this interim monitoring plan are as follows:

- Annual sampling of

- $\quad$ Twelve monitoring points across the site (Figure 1.1) and

- Five outlying IM pilot test monitoring points (PMP4, PMP5, PMP6, PMP7, PMP9; Figure 1.2).

- $\quad$ Twice yearly sampling of five IM pilot test monitoring points inside the injection area (PMP1-PMP3, PMP8, MW02; Figure 1.2).

With the approval of the KDHE (2009), the initial groundwater sampling for VOCs and geochemical analyses under the interim monitoring plan outlined above was conducted in 2009 (Argonne 2010). The present report documents the findings of the 2010 monitoring events, conducted on April 5 and September 19-21, 2010. 


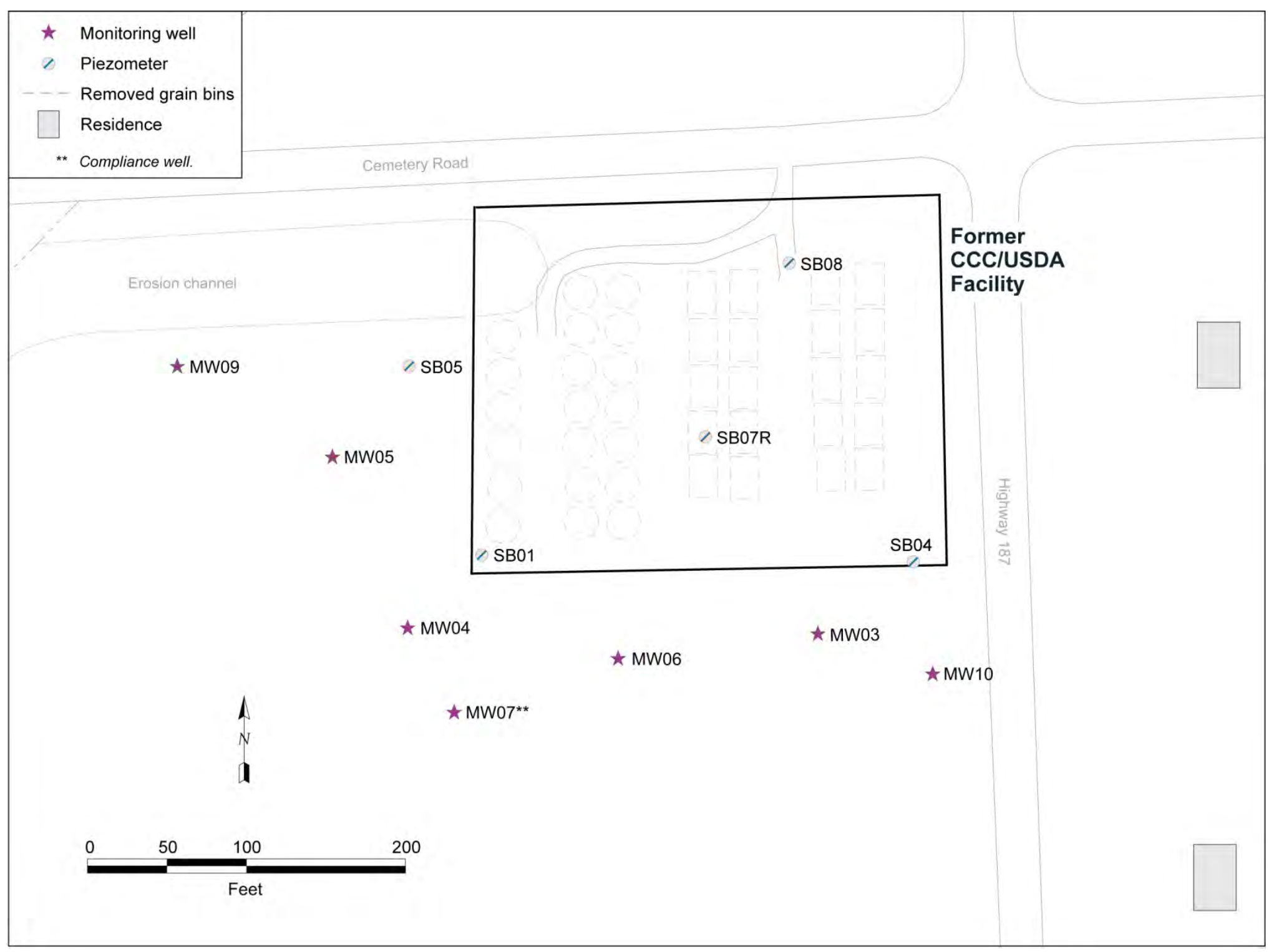




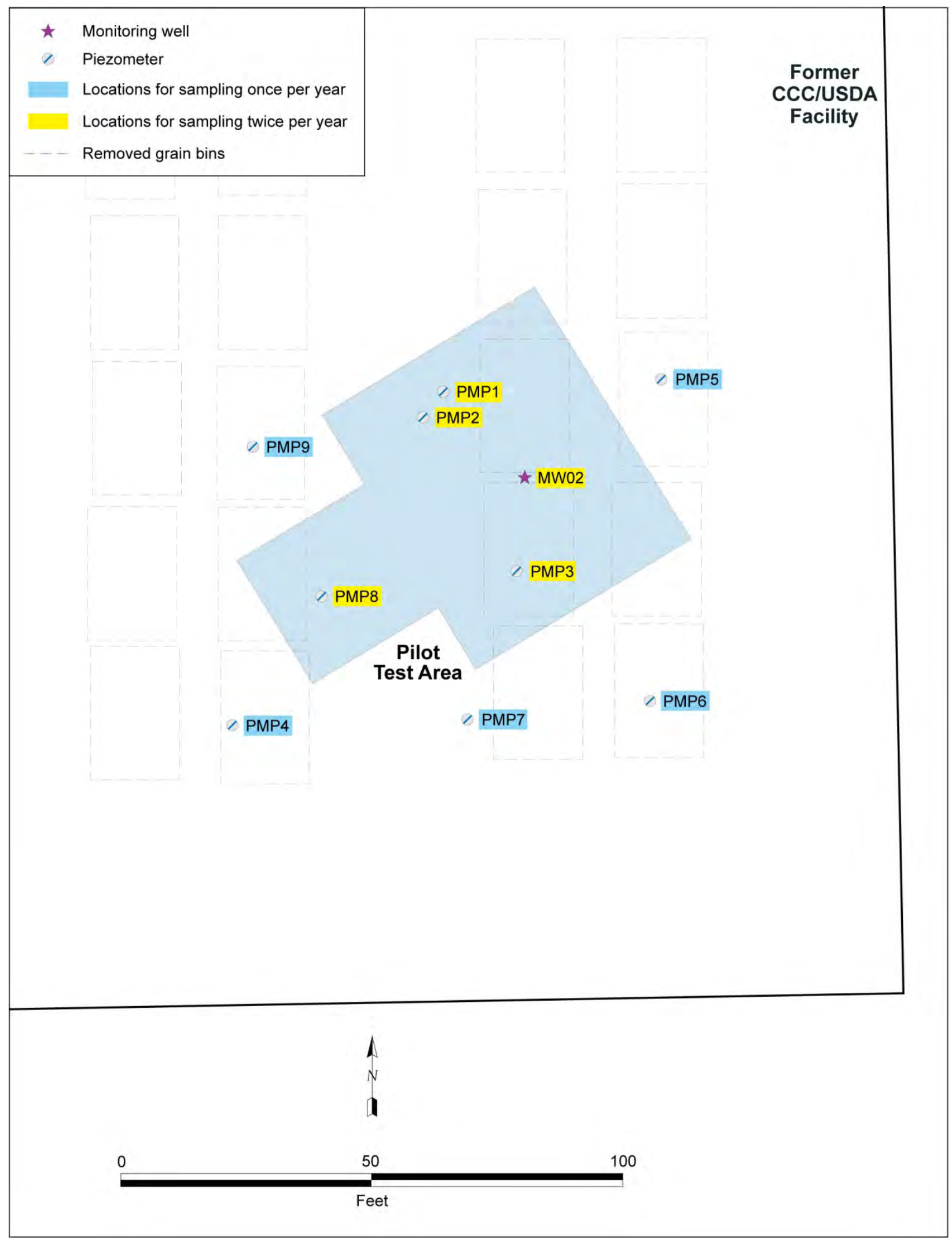

FIGURE 1.2 Pilot test monitoring points currently approved for annual or twice-yearly sampling. 


\section{Sampling and Analysis Activities}

\subsection{Measurement of Groundwater Levels}

Pilot test monitoring points PMP1-PMP3, PMP8, and MW02 (Figure 1.2) were sampled on April 5, 2010. Pilot test monitoring points PMP1-PMP9 and MW02 (Figure 1.2) and sitewide monitoring points MW03-MW07, MW09, MW10, SB01, SB04, SB05, SB07R, and SB08 (Figure 1.1) were sampled on September 19-21, 2010. Before each well or piezometer was sampled, a water level indicator was used to measure the depth to groundwater and the total depth of each well from the top of the well casing.

In wells MW01 and MW03-MW06, downhole pressure sensors equipped with automatic data loggers have been gathering long-term data on the groundwater elevation and gradient. During the current review period, the recorded water level data were retrieved from the loggers on April 28 and September 12, 2010. Water levels were also measured manually in these wells on the same dates as the downloads. In addition, manual water level measurements were made in all of the wells sampled on April 5 and September 19-21, 2010. The groundwater level data are presented and discussed in Section 3.1.

Automated measurement of the groundwater levels began in April 2002, and continuous monitoring of the levels in selected wells has been conducted since August 2004. As outlined in Section 3.1, the results of this program, in conjunction with periodic manual determinations of the water levels in all available monitoring points, have demonstrated long-term consistency in both the groundwater levels and the interpreted patterns of groundwater flow across the investigation site. In light of these findings, automated measurement of the groundwater levels was terminated during the current review period; the pressure sensors and data loggers were removed from the site after final downloads on April 5 and September 12, 2010.

\subsection{Monitoring Well and Piezometer Sampling and Analyses}

After manual measurement of water levels, each monitoring point was purged of a small volume by using a bladder pump or a Waterra pump. With the approval of the KDHE (2008b), the purging was performed by using low-flow techniques in accord with U.S. Environmental Protection Agency (EPA) procedure EPA/540/S-95/504 (Puls and Barcelona 1996) and the 
equipment manufacturers' instructions. Field measurements of temperature, $\mathrm{pH}$, conductivity, dissolved oxygen (DO), and oxidation reduction potential (ORP) were taken during purging until the measurements stabilized. Field measurements of iron(II) and carbon dioxide were made as outlined in the (2005-2007) monitoring plan (Argonne 2005b), in accord with procedures in the Master Work Plan (Argonne 2002). The sequence of activities during each of the 2010 sampling events (in April and September) is summarized in Appendix A, Table A.1.

Groundwater samples designated for VOCs analyses were collected in appropriate laboratory containers, labeled, packaged, and chilled to $4{ }^{\circ} \mathrm{C}$ by placement in ice-filled coolers. The samples were shipped by an overnight delivery service to the Applied Geosciences and Environmental Management (AGEM) Laboratory at Argonne for VOCs analyses by EPA Method 524.2 (EPA 1995). Aliquots of selected samples (chosen in the field) were also shipped to TestAmerica Laboratories, Inc., South Burlington, Vermont, for verification VOCs analyses.

The analytical results for groundwater samples are discussed in Section 3.2.

\subsection{Handling and Disposal of Investigation-Derived Waste}

Purge water generated as potentially contaminated investigation-derived waste was containerized on-site. The accumulated purge water was sampled on September 30, 2010, and analyzed by Pace Analytical Services, Inc., Lenexa, Kansas, on October 3, 2010. Methods used were EPA Method 5030/8260 for VOCs, EPA Method 504.1 for ethylene dibromide, and EPA Method 300 for nitrate as nitrogen. No contamination was detected. The laboratory results are in Supplement 1, on the compact disc (CD) inside the back cover of this report. With the approval of the KDHE, the water was delivered on December 17, 2010 (together with purge water from several other CCC/USDA investigation sites in Kansas), for disposal the Sabetha publicly owned wastewater treatment plant.

\subsection{Quality Control for Sample Collection, Handling, and Analysis}

Quality assurance/quality control procedures followed during the April and September 2010 monitoring events are described in detail in the Master Work Plan (Argonne 2002). The results are summarized as follows: 
- Sample collection and handling activities were monitored by the documentation of samples as they were collected and the use of chain-ofcustody forms and custody seals to ensure sample integrity during handling and shipment.

- Samples designated for VOCs analyses were received with custody seals intact and at the appropriate preservation temperature. All samples were analyzed within the required holding times.

- Quality control samples were all free of carbon tetrachloride and chloroform contamination. These samples included a field blank, equipment rinsates, and trip blanks collected to monitor sample handling activities (Appendix B, Table B.1), as well as method blanks analyzed with the investigation samples to monitor analytical methodologies.

- Groundwater samples were analyzed for VOCs at the AGEM Laboratory with the purge-and-trap method on a gas chromatograph-mass spectrometer system. Calibration checks with each sample delivery group were required to be within $\pm 20 \%$ of the standard. Surrogate standard determinations performed on samples and blanks were within the specified range of $80-120 \%$ for all samples, in either the initial analysis or a successful reanalysis.

- Accuracy and precision of the analytical methodology were evident in the analysis of two replicate samples and the duplicate analysis of three additional samples, with average relative percent difference values of $2 \%$ between the initial analysis and the associated quality control analysis for both carbon tetrachloride and chloroform (Table B.1 in Appendix B). The groundwater analytical data from the AGEM Laboratory are acceptable for quantitative determination of contaminant distribution.

- In accordance with the quality control procedures defined in the Master Work Plan (Argonne 2002), the analyses of water samples at the AGEM Laboratory were verified by a second laboratory. Three groundwater samples collected during the April 2010 monitoring event (from MW02, PMP2, and PMP3) and four samples from the September 2010 event (from MW06, MW07, MW08, 
and SB09) were submitted to TestAmerica for verification organic analysis according to EPA Contract Laboratory Program methodology. Results (Table B.2 in Appendix B) showed good agreement over the range of contaminant concentrations detected, with average relative percent difference values of $<10 \%$ for both carbon tetrachloride and chloroform. The detection of methylene chloride, a secondary dechlorination by-product of carbon tetrachloride, was confirmed in the verification analyses. The verification organic analyses are on compact disc, in Supplement 2. 


\section{Results and Discussion}

\subsection{Groundwater Level Data}

Depths to groundwater were measured manually in each of the wells sampled during the monitoring events on April 5 and September 19-21, 2010. Water levels were also measured manually in conjunction with the final data logger downloads on April 28 and September 12, 2010. The hand-measured water level data are in Table 3.1.

Hydrographs depicting the variations in water levels in monitored wells MW01 and MW03-MW06 during the current (2010) and previous (2009) review periods are in Figure 3.1. The water level traces are shown in conjunction with daily precipitation data obtained from the Kansas State University recording weather station in Powhattan, Kansas, approximately $26 \mathrm{mi}$ east of Centralia (http://wdl.agron.ksu.edu/). Figure 3.1 indicates that the groundwater levels at Centralia have fluctuated by approximately $0.5-5 \mathrm{ft}$ in response to both seasonal and shorter-term rainfall events but showed little net change in 2009-2010. The pronounced, transient water level "spikes" indicated in the hydrograph for monitoring well MW06 are believed to reflect localized flooding at the location of this (flush-mounted) monitoring well that occurs during heavy rainfall events, particularly in the spring and early summer. These observations are consistent with the results of the continuous automated monitoring conducted in selected wells since August 2004 (Argonne 2006, 2007a, 2008a,b, 2009b, 2010).

The potentiometric surface at Centralia, as determined from manual measurements on April 28, 2010, is depicted in Figure 3.2. The recent results are consistent with previous interpretations (Argonne 2006, 2007a, 2008a,b, 2009b, 2010), indicating an apparent groundwater flow direction toward the southwest across much of the former CCC/USDA facility. Like previous depictions, Figure 3.2 indicates that groundwater flow appears focused toward a localized low in the potentiometric surface, defined by the water level measurements at SB01, MW04, MW06, and MW07. Argonne's earlier investigations (Argonne 2003, 2004) suggested that the increased hydraulic gradients observed near these wells are a reflection of relatively low-permeability silts and clays that compose the aquifer unit in this portion of the study area, in comparison to the coarser-grained deposits identified in the northern and eastern portions of the site. The results of the sitewide groundwater analyses discussed in Section 3.2.1 support an interpretation of slow groundwater flow (and carbon tetrachloride migration) to the south-southwest, in keeping with the observed water level patterns. 


\subsection{Groundwater Analysis Results}

In September 2010, sitewide groundwater sampling was performed, with the approval of the KDHE (2009), in a suite of 12 monitoring points (Figure 1.1). More detailed sampling in the IM pilot test area was conducted in April and September 2010, in the wells identified in Figure 1.2. The results of the 2010 sitewide (September) and IM pilot test area (April and September) monitoring efforts are summarized, respectively, in Section 3.2.1 and Section 3.2.2.

\subsubsection{Sitewide Monitoring Results}

The analytical data for VOCs in the groundwater samples collected in the network of sitewide monitoring wells in September 2010 are in Table 3.2, together with data generated since sampling of the monitoring wells began in 2004. The September 2010 data for carbon tetrachloride are illustrated in Figure 3.3, along with the lateral margins of the contaminant distribution, as interpreted on the basis of each of the sitewide groundwater sampling events summarized in Table 3.2.

Carbon tetrachloride was detected in September 2010 at 9 of the 12 sitewide monitoring locations on and downgradient from the former CCC/USDA facility (Figure 3.3), at concentrations ranging from $2.2 \mu \mathrm{g} / \mathrm{L}$ (at MW04) to a maximum of $374 \mu \mathrm{g} / \mathrm{L}$ (at SB05). Chloroform concentrations ranging from $<1 \mu \mathrm{g} / \mathrm{L}$ to $32 \mu \mathrm{g} / \mathrm{L}$ were detected at 8 of the 12 sampled locations (Table 3.2).

The carbon tetrachloride concentrations identified in the sitewide monitoring wells in September 2010 were generally comparable to the measurements obtained in the previous (2009) monitoring period, with individual wells showing only minor changes. The results in Table 3.2 and Figure 3.3 continue to indicate the longer-term trends (observed previously) of slightly increasing carbon tetrachloride levels at monitoring points SB05, MW04, MW07, and (since 2008) MW05 along the western and southern margins of the groundwater plume and in the apparent direction of groundwater flow.

The results of field measurements on the groundwater samples from wells in the sitewide monitoring network are summarized in Table 3.3. The presence of trace to relatively low levels of chloroform at all of the monitoring points (except for MW04) having detectable levels of 
carbon tetrachloride (Table 3.2) suggests that some degradation of carbon tetrachloride is occurring at these locations. The relatively high DO concentrations (2.48-10.48 mg/L) and positive ORP levels (60 mV to $186 \mathrm{mV}$ ) identified at the sitewide monitoring points (Table 3.3) do not, however, support the widespread occurrence of anaerobic reducing conditions within the Centralia aquifer.

Table 3.3 indicates that the identified DO concentrations and ORP levels at monitoring well MW06 have fluctuated erratically since the monitoring of these parameters began in 2004. Low DO concentrations (<1 mg/L) and negative ORP levels (-72 to $-96 \mathrm{mV}$ ) detected at MW06 in September 2008 and October 2009 (Table 3.3), were interpreted as possibly suggesting the transient development of increasingly anaerobic reducing conditions at this location; however, these results were not reproduced in the current review period.

\subsubsection{Monitoring Results for the IM Pilot Test Area}

Baseline groundwater sampling was conducted within and adjacent to the IM pilot test area in September and November 2007, prior to the injection of the ISCR materials, to provide a basis for assessment of the ISCR treatment technology over time. The pre-treatment concentrations of carbon tetrachloride and the values of DO and ORP identified during this sampling (Argonne 2009a) are illustrated in Figures 3.4-3.6, respectively.

Injection of the ISCR materials (in November-December 2007) initially generated extremely reducing, oxygen-depleted groundwater conditions (conducive to the reductive dechlorination of carbon tetrachloride) within the injection field, while less dramatic reductions in DO and ORP were observed at monitoring points outside the treatment area. The extremely low DO and ORP levels were, however, maintained for only approximately 5-7 weeks after injection. Subsequent monitoring in 2008 (Argonne 2009a,b) demonstrated that the DO and ORP levels within the injection field remained consistently lower than those at monitoring points outside the injection area, but the results showed no clear indication of further geochemical effects beyond the limits of the injection field.

Reductions of $96-99 \%$ in the concentrations of carbon tetrachloride in groundwater within the injection field and of $20-70 \%$ at most monitoring points near the injection area were observed in the first 5-7 weeks after injection. Continued monitoring in 2008 showed that carbon 
tetrachloride concentrations in the injection field generally remained near the initial postinjection levels or decreased slightly more, while the concentrations at points bordering or outside the injection area showed little consistency and variably decreased, increased, or remained relatively unchanged (Argonne 2009a) after the initial 5-7 weeks following the injection.

The analytical data for VOCs in the groundwater samples collected from the IM pilot test monitoring points (PMP1-PMP9 and MW02; Figure 1.2) in April and September 2010 are in Table 3.4, together with data for the most recent previous sampling events (October 2009, April 2009, and September 2008) at these locations. The corresponding field measurements for these locations and sampling events are in Table 3.5. Time series diagrams summarizing the complete sequence of analysis results for selected parameters (carbon tetrachloride, chloroform, methylene chloride, DO, ORP) at each IM monitoring point since the ISCR pilot test was implemented in November 2007 are in Appendix C, Figures C.1-C.10.

Carbon tetrachloride was detected at 3 of the 5 points sampled in the pilot test area during the April 2010 monitoring event and at 9 of the 10 points sampled in September 2010. In April 2010, carbon tetrachloride concentrations ranging from $<1 \mu \mathrm{g} / \mathrm{L}$ to $991 \mu \mathrm{g} / \mathrm{L}$ were identified at PMP1, PMP2, and PMP8. In September 2010, concentrations ranging from < $1 \mu \mathrm{g} / \mathrm{L}$ (at PMP8) to $779 \mu \mathrm{g} / \mathrm{L}$ (at PMP5) were detected at monitoring well MW02 and piezometers PMP1, PMP2, and PMP4-PMP9 (Table 3.4). No carbon tetrachloride was detected at monitoring point PMP3 during either 2010 sampling event.

The results of the September 2010 and October 2009 analyses for carbon tetrachloride are compared in Figure 3.7. The carbon tetrachloride concentrations in groundwater at monitoring points PMP1-PMP4, PMP6, PMP8, and PMP9 decreased from October 2009 to September 2010, with the most significant decrease during this period occurring at point PMP2 (from 1,384 $\mu \mathrm{g} / \mathrm{L}$ in October 2009 to $117 \mu \mathrm{g} / \mathrm{L}$ in September 2010). The concentrations at MW02, PMP5, and PMP7 increased slightly from October 2009 to September 2010. These relatively short-term variations in carbon tetrachloride levels (from October 2009 to September 2010; Figure 3.7) in several cases do not appear representative, however, of possible longer-term trends in the contaminant concentrations at these monitoring points. The time series diagrams (Figures C.1-C.10 in Appendix C) suggest a net increase in the concentrations of carbon tetrachloride identified at locations PMP5, PMP6, and PMP9 (lying to the east and northwest of the pilot test injection field), and a net decrease in the concentrations at points PMP4 and PMP7 
(lying to the southwest and downgradient of the injection field) since the September 2008 sampling event. Except for PMP2, the carbon tetrachloride concentrations at monitoring points in the injection field (MW02, PMP1, PMP3, and PMP8) gave little indication of increasing or decreasing trends during the 2009 and 2010 sampling events.

The DO concentrations and ORP levels identified in the pilot test area in September 2010 and October 2009 are summarized in Table 3.5 and Figures 3.8 and 3.9, respectively. Figure 3.9 and Figures C.1-C.10 in Appendix C illustrate that the observed ORP levels in and near the pilot test injection field remained relatively stable throughout 2009 and 2010. Consistently lower (and predominantly negative) ORP values have persisted in the injection field relative to the levels observed at the nearby monitoring points outside this area, demonstrating the apparent continued, localized influence of the ISCR treatment. Similarly, DO levels at monitoring points within the ISCR injection field (Table 3.5, Figure 3.8, and Figures C.1-C.10) have remained consistently lower, although somewhat more variable, than those at the nearby monitoring points immediately outside this area (with the possible exception of PMP7).

Relatively high levels of chloroform (relative to carbon tetrachloride; Table 3.4 and graphs in Appendix C) were also observed at PMP1, PMP2, and PMP4-PMP7 in the 2010 sampling events, and low levels of methylene chloride were detected at three of the pilot test monitoring locations (PMP2, PMP5, PMP7). Together, these findings confirm that geochemical conditions favorable to the degradation of carbon tetrachloride, via reductive dechlorination, persist in the pilot test area as a result of the November 2007 ISCR injections.

Data discussed previously (Argonne 2010) indicated that DO and ORP values decreased from September 2008 to October 2009 at monitoring points PMP4, PMP6, PMP7, and PMP9 immediately to the south, west, and downgradient of the pilot test injection field. Slightly lower concentrations of carbon tetrachloride were also identified at the PMP4 and PMP7 locations in October 2009 (Table 3.4). These relationships empirically suggested possible slow expansion of the range of influence of the ISCR treatment technology with time, in the direction of natural groundwater flow to the southwest. Additional monitoring in the pilot test area will be necessary, however, to substantiate this hypothesis, as the suggestion of coupled geochemical and concentration trends could not be confirmed on the basis of the 2010 monitoring results. 
TABLE 3.1 Hand-measured water levels at Centralia in 2010.

\begin{tabular}{|c|c|c|c|c|c|c|c|c|c|}
\hline \multirow[b]{2}{*}{ Well } & \multirow[b]{2}{*}{$\begin{array}{l}\text { Top of } \\
\text { Casing } \\
\text { Elevation } \\
\text { (ft AMSL) }\end{array}$} & \multicolumn{2}{|c|}{ April $5,2010^{a}$} & \multicolumn{2}{|c|}{ April 28, 2010} & \multicolumn{2}{|c|}{ September 12, 2010} & \multicolumn{2}{|c|}{ September $19-21,2010^{a}$} \\
\hline & & $\begin{array}{l}\text { Depth to } \\
\text { Groundwaterc } \\
\text { (ft TOC) }\end{array}$ & $\begin{array}{l}\text { Groundwater } \\
\text { Elevation } \\
\text { (ft AMSL) }\end{array}$ & $\begin{array}{l}\text { Depth to } \\
\text { Groundwater } \\
\text { (ft TOC) }\end{array}$ & $\begin{array}{l}\text { Groundwater } \\
\text { Elevation } \\
\text { (ft AMSL) }\end{array}$ & $\begin{array}{l}\text { Depth to } \\
\text { Groundwater } \\
\text { (ft TOC) }\end{array}$ & $\begin{array}{l}\text { Groundwater } \\
\text { Elevation } \\
\text { (ft AMSL) }\end{array}$ & $\begin{array}{l}\text { Depth to } \\
\text { Groundwater } \\
\text { (ft TOC) }\end{array}$ & $\begin{array}{l}\text { Groundwater } \\
\text { Elevation } \\
\text { (ft AMSL) }\end{array}$ \\
\hline MW01 & 1329.17 & & & 9.97 & 1319.20 & 12.65 & 1316.52 & 12.17 & 1317.00 \\
\hline MW02 & 1334.67 & 18.70 & 1315.97 & 18.08 & 1316.59 & & & 19.72 & 1314.95 \\
\hline MW03 & 1334.51 & & & 17.88 & 1316.63 & 19.37 & 1315.14 & 19.42 & 1315.09 \\
\hline MW04 & 1322.57 & & & 20.88 & 1301.69 & 22.35 & 1300.22 & 22.42 & 1300.15 \\
\hline MW05 & 1317.97 & & & 7.93 & 1310.04 & 11.06 & 1306.91 & 10.38 & 1307.59 \\
\hline MW06 & 1329.63 & & & 34.27 & 1295.36 & 34.54 & 1295.09 & 34.96 & 1294.67 \\
\hline MW07 & 1324.76 & & & 24.75 & 1300.01 & & & 25.03 & 1299.73 \\
\hline MW08 & 1332.34 & & & 15.76 & 1316.58 & & & 17.52 & 1314.82 \\
\hline MW09 & 1310.41 & & & 0.20 & 1310.21 & & & 2.66 & 1307.75 \\
\hline MW10 & 1334.39 & & & 17.73 & 1316.66 & & & 19.92 & 1314.47 \\
\hline SB01 & 1325.15 & & & 14.07 & 1311.08 & & & 14.02 & 1311.13 \\
\hline SB04 & 1335.67 & & & 19.02 & 1316.65 & & & 20.42 & 1315.25 \\
\hline SB05 & 1321.28 & & & 6.75 & 1314.53 & & & 10.20 & 1311.08 \\
\hline SB07R & 1331.57 & & & 14.95 & 1316.62 & & & 17.19 & 1314.38 \\
\hline SB08 & 1332.48 & & & 15.81 & 1316.67 & & & 17.23 & 1315.25 \\
\hline SB09 & 1311.07 & & & 4.36 & 1306.71 & & & 6.78 & 1304.29 \\
\hline PMP1 & 1333.70 & 17.25 & 1316.45 & & & & & 18.65 & 1315.05 \\
\hline PMP2 & 1333.67 & 17.16 & 1316.51 & & & & & 18.68 & 1314.99 \\
\hline PMP3 & 1334.57 & 17.90 & 1316.67 & & & & & 19.35 & 1315.22 \\
\hline PMP4 & 1331.99 & & & & & & & 16.83 & 1315.16 \\
\hline PMP5 & 1335.07 & & & & & & & 20.20 & 1314.87 \\
\hline PMP6 & 1335.19 & & & & & & & 20.00 & 1315.19 \\
\hline PMP7 & 1334.06 & & & & & & & 18.84 & 1315.22 \\
\hline PMP8 & 1332.94 & 16.12 & 1316.82 & & & & & 17.91 & 1315.03 \\
\hline PMP9 & 1331.83 & & & & & & & 15.30 & 1316.53 \\
\hline
\end{tabular}

a Measurements made during sampling.

b 2009 surveyed elevations.

c Depths measured from the top of casing (TOC). 
TABLE 3.2 Analytical results from the AGEM Laboratory for volatile organic compounds in groundwater samples collected from the sitewide monitoring points at Centralia, August 2004 to September 2010.

\begin{tabular}{|c|c|c|c|c|c|c|}
\hline \multirow[b]{2}{*}{ Well } & \multirow[b]{2}{*}{$\begin{array}{l}\text { Screen } \\
\text { Interval } \\
\text { (ft BGL) }\end{array}$} & \multirow[b]{2}{*}{ Sample } & \multirow[b]{2}{*}{$\begin{array}{l}\text { Sample } \\
\text { Date }\end{array}$} & \multicolumn{3}{|c|}{ Concentration $(\mu \mathrm{g} / \mathrm{L})$} \\
\hline & & & & $\begin{array}{c}\text { Carbon } \\
\text { Tetrachloride }\end{array}$ & Chloroform & $\begin{array}{c}\text { Methylene } \\
\text { Chloride }\end{array}$ \\
\hline \multirow{9}{*}{ MW01 } & $54.5-64.5$ & CNMW01-W-16158 & 8/24/04 & $N^{a}$ & ND & ND \\
\hline & & CNMW01-W-19276 & 9/10/05 & ND & ND & ND \\
\hline & & CNMW01-W-16308 & $10 / 11 / 05$ & ND & ND & ND \\
\hline & & CNMW01-W-19890 & $3 / 15 / 06$ & ND & ND & ND \\
\hline & & CNMW01-W-22501 & $9 / 25 / 06$ & ND & ND & ND \\
\hline & & CNMW01-W-16326 & $3 / 29 / 07$ & ND & ND & ND \\
\hline & & CNMW01-W-16228 & $9 / 26 / 07$ & $1.0 \mathrm{R}^{\mathrm{b}}$ & ND & ND \\
\hline & & CNMW01-W-26023 & $3 / 19 / 08$ & ND & ND & ND \\
\hline & & CNMW01-W-26673 & 9/9/08 & ND & ND & ND \\
\hline \multirow[t]{7}{*}{$\mathrm{MWO2}^{\mathrm{C}}$} & $49.5-59.5$ & CNMW02-W-16159 & $8 / 26 / 04$ & 215 & 6.2 & ND \\
\hline & & CNMW02-W-19282 & $9 / 11 / 05$ & 776 & 33 & ND \\
\hline & & CNMW02-W-16309 & $10 / 12 / 05$ & 528 & 21 & ND \\
\hline & & CNMW02-W-19908 & $3 / 16 / 06$ & 847 & 21 & ND \\
\hline & & CNMW02-W-22508 & $9 / 26 / 06$ & 1233 & 25 & ND \\
\hline & & CNMW02-W-15489 & $3 / 26 / 07$ & 829 & 14 & ND \\
\hline & & CNMW02-W-16227 & $9 / 26 / 07$ & 1138 & 18 & ND \\
\hline \multirow[t]{11}{*}{ MW03 } & $50.5-60.5$ & CNMW03-W-16178 & $8 / 24 / 04$ & 1.2 & ND & ND \\
\hline & & CNMW03-W-19277 & 9/10/05 & 1.6 & ND & ND \\
\hline & & CNMW03-W-16310 & $10 / 11 / 05$ & 1.8 & ND & ND \\
\hline & & CNMW03-W-19909 & $3 / 17 / 06$ & 2.6 & $0.2 \mathrm{Jd}^{\mathrm{d}}$ & ND \\
\hline & & CNMW03-W-22513 & $9 / 26 / 06$ & 2.7 & ND & ND \\
\hline & & CNMW03-W-15494 & $3 / 27 / 07$ & 2.5 & ND & ND \\
\hline & & CNMW03-W-16223 & $9 / 25 / 07$ & 3.5 & ND & ND \\
\hline & & CNMW03-W-26001 & $3 / 12 / 08$ & 2.3 & ND & ND \\
\hline & & CNMW03-W-26675 & $9 / 9 / 08$ & 3.2 & $0.3 \mathrm{~J}$ & ND \\
\hline & & CNMW03-W-27151 & 10/6/09 & 6.2 & ND & ND \\
\hline & & CNMW03-W-27188 & $9 / 19 / 10$ & 7.5 & $0.3 \mathrm{~J}$ & ND \\
\hline \multirow[t]{11}{*}{ MW04 } & $37.5-47.5$ & CNMW04-W-16180 & $8 / 24 / 04$ & ND & ND & ND \\
\hline & & CNMW04-W-19280 & $9 / 11 / 05$ & $0.9 \mathrm{~J}$ & ND & ND \\
\hline & & CNMW04-W-16311 & $10 / 11 / 05$ & $0.8 \mathrm{~J}$ & ND & ND \\
\hline & & CNMW04-W-19891 & $3 / 15 / 06$ & 1.3 & ND & ND \\
\hline & & CNMW04-W-22506 & $9 / 25 / 06$ & 1.4 & $0.1 \mathrm{~J}$ & ND \\
\hline & & CNMW04-W-16210 & $3 / 28 / 07$ & 2.1 & ND & ND \\
\hline & & CNMW04-W-16220 & $9 / 24 / 07$ & 2.0 & ND & ND \\
\hline & & CNMW04-W-26024 & $3 / 19 / 08$ & 1.3 & ND & ND \\
\hline & & CNMW04-W-26676 & 9/9/08 & 2.0 & ND & ND \\
\hline & & CNMW04-W-27152 & 10/7/09 & 2.9 & ND & ND \\
\hline & & CNMW04-W-27189 & $9 / 20 / 10$ & 2.2 & ND & ND \\
\hline
\end{tabular}


TABLE 3.2 (Cont.)

\begin{tabular}{|c|c|c|c|c|c|c|}
\hline \multirow[b]{2}{*}{ Well } & \multirow[b]{2}{*}{$\begin{array}{l}\text { Screen } \\
\text { Interval } \\
\text { (ft BGL) }\end{array}$} & \multirow[b]{2}{*}{ Sample } & \multirow[b]{2}{*}{$\begin{array}{l}\text { Sample } \\
\text { Date }\end{array}$} & \multicolumn{3}{|c|}{ Concentration ( $\mu \mathrm{g} / \mathrm{L})$} \\
\hline & & & & $\begin{array}{c}\text { Carbon } \\
\text { Tetrachloride }\end{array}$ & Chloroform & $\begin{array}{l}\text { Methylene } \\
\text { Chloride }\end{array}$ \\
\hline \multirow{11}{*}{ MW05 } & $34.5-44.5$ & CNMW05-W-16183 & 8/25/04 & ND & ND & ND \\
\hline & & CNMW05-W-19279 & 9/10/05 & 1.9 & ND & ND \\
\hline & & CNMW05-W-16312 & $10 / 11 / 05$ & 1.5 & ND & ND \\
\hline & & CNMW05-W-19976 & 3/15/06 & 1.3 & ND & ND \\
\hline & & CNMW05-W-22505 & $9 / 25 / 06$ & 1.3 & ND & ND \\
\hline & & CNMW05-W-16213 & $3 / 28 / 07$ & $0.5 \mathrm{~J}$ & ND & ND \\
\hline & & CNMW05-W-16218 & $9 / 24 / 07$ & 1.2 & ND & ND \\
\hline & & CNMW05-W-26025 & $3 / 19 / 08$ & 1.9 & ND & ND \\
\hline & & CNMW05-W-26677 & 9/10/08 & 13 & $0.7 \mathrm{~J}$ & ND \\
\hline & & CNMW05-W-27153 & $10 / 7 / 09$ & 18 & 1.1 & ND \\
\hline & & CNMW05-W-27190 & 9/20/10 & 22 & 1.4 & ND \\
\hline \multirow[t]{11}{*}{ MW06 } & $46.5-56.5$ & CNMW06-W-16184 & $8 / 25 / 04$ & ND & ND & ND \\
\hline & & CNMW06-W-19278 & 9/10/05 & ND & ND & ND \\
\hline & & CNMW06-W-16313 & $10 / 11 / 05$ & $0.3 \mathrm{~J}$ & ND & ND \\
\hline & & CNMW06-W-19889 & 3/15/06 & $0.2 \mathrm{~J}$ & ND & ND \\
\hline & & CNMW06-W-22511 & $9 / 27 / 06$ & ND & ND & ND \\
\hline & & CNMW06-W-16208 & $3 / 27 / 07$ & ND & ND & ND \\
\hline & & CNMW06-W-16222 & $9 / 24 / 07$ & ND & ND & ND \\
\hline & & CNMW06-W-26026 & $3 / 19 / 08$ & ND & ND & ND \\
\hline & & CNMW06-W-26678 & 9/9/08 & ND & ND & ND \\
\hline & & CNMW06-W-27154 & 10/6/09 & ND & ND & ND \\
\hline & & CNMW06-W-27191 & $9 / 20 / 10$ & ND & ND & ND \\
\hline \multirow[t]{8}{*}{ MW07 } & $45-55$ & CNMW07-W-19887 & 3/14/06 & $0.4 \mathrm{~J}$ & $0.6 \mathrm{~J}$ & ND \\
\hline & & CNMW07-W-22512 & 9/26/06 & 1.1 & ND & ND \\
\hline & & CNMW07-W-15492 & $3 / 26 / 07$ & 1.8 & ND & ND \\
\hline & & CNMW07-W-16221 & $9 / 24 / 07$ & 2.4 & ND & ND \\
\hline & & CNMW07-W-26027 & $3 / 19 / 08$ & 3.0 & ND & ND \\
\hline & & CNMW07-W-26679 & 9/9/08 & 4.0 & $0.2 \mathrm{~J}$ & ND \\
\hline & & CNMW07-W-27155 & 10/6/09 & 5.1 & $0.6 \mathrm{~J}$ & ND \\
\hline & & CNMW07-W-27192 & $9 / 20 / 10$ & 6.6 & $0.3 \mathrm{~J}$ & ND \\
\hline \multirow[t]{6}{*}{ MW08 } & $38-53$ & CNMW08-W-19284 & $3 / 14 / 06$ & ND & ND & ND \\
\hline & & CNMW08-W-22507 & $9 / 26 / 06$ & ND & ND & ND \\
\hline & & CNMW08-W-15493 & $3 / 27 / 07$ & ND & ND & ND \\
\hline & & CNMW08-W-16226 & $9 / 25 / 07$ & ND & ND & ND \\
\hline & & CNMW08-W-26028 & $3 / 20 / 08$ & ND & ND & ND \\
\hline & & CNMW08-W-26680 & 9/10/08 & ND & ND & ND \\
\hline \multirow[t]{8}{*}{ MW09 } & $25-35$ & CNMW09-W-19285 & $3 / 15 / 06$ & ND & ND & ND \\
\hline & & CNMW09-W-22504 & $9 / 25 / 06$ & ND & ND & ND \\
\hline & & CNMW09-W-16209 & $3 / 27 / 07$ & ND & ND & ND \\
\hline & & CNMW09-W-16219 & $9 / 24 / 07$ & ND & ND & ND \\
\hline & & CNMW09-W-26029 & $3 / 20 / 08$ & ND & ND & ND \\
\hline & & CNMW09-W-26681 & 9/10/08 & ND & ND & ND \\
\hline & & CNMW09-W-27157 & $10 / 6 / 09$ & ND & ND & ND \\
\hline & & CNMW09-W-27194 & 9/19/10 & ND & ND & ND \\
\hline
\end{tabular}


TABLE 3.2 (Cont.)

\begin{tabular}{|c|c|c|c|c|c|c|}
\hline \multirow[b]{2}{*}{ Well } & \multirow[b]{2}{*}{$\begin{array}{l}\text { Screen } \\
\text { Interval } \\
\text { (ft BGL) }\end{array}$} & \multirow[b]{2}{*}{ Sample } & \multirow[b]{2}{*}{$\begin{array}{l}\text { Sample } \\
\text { Date }\end{array}$} & \multicolumn{3}{|c|}{ Concentration $(\mu \mathrm{g} / \mathrm{L})$} \\
\hline & & & & $\begin{array}{c}\text { Carbon } \\
\text { Tetrachloride }\end{array}$ & Chloroform & $\begin{array}{c}\text { Methylene } \\
\text { Chloride }\end{array}$ \\
\hline \multirow[t]{8}{*}{ MW10 } & $30-45$ & CNMW10-W-19886 & $3 / 14 / 06$ & ND & ND & ND \\
\hline & & CNMW10-W-22510 & 9/26/06 & ND & ND & ND \\
\hline & & CNMW10-W-16215 & $3 / 28 / 07$ & ND & ND & ND \\
\hline & & CNMW10-W-16224 & 9/25/07 & ND & ND & ND \\
\hline & & CNMW10-W-26030 & 3/20/08 & ND & ND & ND \\
\hline & & CNMW10-W-26682 & 9/9/08 & ND & ND & ND \\
\hline & & CNMW10-W-27158 & 10/6/09 & ND & ND & ND \\
\hline & & CNMW10-W-27195 & 9/19/10 & ND & ND & ND \\
\hline \multirow[t]{11}{*}{ SB01 } & $40-50$ & CNSB01-W-16188 & $8 / 26 / 04$ & 186 & 6.5 & ND \\
\hline & & CNSB01-W-19274 & 9/9/05 & 269 & 6.8 & ND \\
\hline & & CNSB01-W-16314 & 10/12/05 & 288 & 6.6 & ND \\
\hline & & CNSB01-W-19979 & $3 / 17 / 06$ & 320 & 5.7 & ND \\
\hline & & CNSB01-W-22516 & 9/27/06 & 267 & 6.3 & ND \\
\hline & & CNSB01-W-15491 & $3 / 27 / 07$ & 222 & 4.9 & ND \\
\hline & & CNSB01-W-16232 & 9/27/07 & 283 & 4.6 & ND \\
\hline & & CNSB01-W-26031 & 3/20/08 & 325 & 4.8 & ND \\
\hline & & CNSB01-W-26683 & 9/10/08 & 378 & 4.1 & ND \\
\hline & & CNSB01-W-27159 & $10 / 7 / 09$ & 396 & 5.0 & ND \\
\hline & & CNSB01-W-27196 & $9 / 20 / 10$ & 319 & 4.7 & ND \\
\hline \multirow[t]{11}{*}{ SB04 } & $51-61$ & CNSB04-W-16189 & 8/26/04 & 30 & ND & ND \\
\hline & & CNSB04-W-19273 & 9/9/05 & 47 & $0.6 \mathrm{~J}$ & ND \\
\hline & & CNSB04-W-16315 & $10 / 12 / 05$ & 44 & $0.5 \mathrm{~J}$ & ND \\
\hline & & CNSB04-W-19906 & $3 / 16 / 06$ & 51 & $0.5 \mathrm{~J}$ & $0.4 \mathrm{~J} \mathrm{~B}^{\mathrm{e}}$ \\
\hline & & CNSB04-W-22503 & 9/25/06 & 54 & $0.7 \mathrm{~J}$ & ND \\
\hline & & CNSB04-W-16216 & $3 / 28 / 07$ & 44 & $0.5 \mathrm{~J}$ & ND \\
\hline & & CNSB04-W-16230 & 9/26/07 & 36 & $0.4 \mathrm{~J}$ & ND \\
\hline & & CNSB04-W-26002 & 3/12/08 & 30 & $0.3 \mathrm{~J}$ & ND \\
\hline & & CNSB04-W-26684 & 9/9/08 & 15 & $0.3 \mathrm{~J}$ & ND \\
\hline & & CNSB04-W-27160 & $10 / 8 / 09$ & 17 & $0.3 \mathrm{~J}$ & ND \\
\hline & & CNSB04-W-27197 & 9/20/10 & 17 & $0.3 \mathrm{~J}$ & ND \\
\hline \multirow[t]{11}{*}{ SB05 } & $32-42$ & CNSB05-W-16190 & 8/26/04 & 59 & 5.5 & ND \\
\hline & & CNSB05-W-19275 & 9/9/05 & 77 & 7.2 & ND \\
\hline & & CNSB05-W-16323 & $10 / 12 / 05$ & 54 & 5.5 & ND \\
\hline & & CNSB05-W-19904 & $3 / 17 / 06$ & 104 & 7.2 & ND \\
\hline & & CNSB05-W-19940 & 9/27/06 & 139 & 12 & ND \\
\hline & & CNSB05-W-16212 & 3/28/07 & 138 & 12 & ND \\
\hline & & CNSB05-W-16233 & 9/26/07 & 221 & 16 & ND \\
\hline & & CNSB05-W-26032 & 3/20/08 & 224 & 17 & ND \\
\hline & & CNSB05-W-26685 & 9/9/08 & 256 & 20 & ND \\
\hline & & CNSB05-W-27161 & $10 / 8 / 09$ & 289 & 19 & ND \\
\hline & & CNSB05-W-27198 & $9 / 21 / 10$ & 374 & 32 & ND \\
\hline \multirow[t]{8}{*}{ SB07R } & $45-60$ & CNSB07R-W-19978 & 3/15/06 & 41 & 2.7 & ND \\
\hline & & CNSB07R-W-19924 & $9 / 26 / 06$ & 30 & 1.7 & ND \\
\hline & & CNSB07R-W-15490 & 3/26/07 & 30 & 1.7 & ND \\
\hline & & CNSB07R-W-16225 & 9/25/07 & 50 & 2.4 & ND \\
\hline & & CNSB07R-W-26003 & $3 / 12 / 08$ & 13 & $0.9 \mathrm{~J}$ & ND \\
\hline & & CNSB07R-W-26686 & 9/9/08 & 21 & 1.4 & ND \\
\hline & & CNSB07R-W-27162 & $10 / 7 / 09$ & 38 & 1.7 & ND \\
\hline & & CNSB07R-W-27199 & 9/20/10 & 42 & 2.5 & ND \\
\hline
\end{tabular}


TABLE 3.2 (Cont.)

\begin{tabular}{|c|c|c|c|c|c|c|}
\hline \multirow[b]{2}{*}{ Well } & \multirow[b]{2}{*}{$\begin{array}{l}\text { Screen } \\
\text { Interval } \\
\text { (ft BGL) }\end{array}$} & \multirow[b]{2}{*}{ Sample } & \multirow[b]{2}{*}{$\begin{array}{c}\text { Sample } \\
\text { Date }\end{array}$} & \multicolumn{3}{|c|}{ Concentration $(\mu \mathrm{g} / \mathrm{L})$} \\
\hline & & & & $\begin{array}{c}\text { Carbon } \\
\text { Tetrachloride }\end{array}$ & Chloroform & $\begin{array}{c}\text { Methylene } \\
\text { Chloride }\end{array}$ \\
\hline \multirow[t]{11}{*}{ SB08 } & $52-62$ & CNSB08-W-16192 & $8 / 26 / 04$ & 79 & 3.1 & ND \\
\hline & & CNSB08-W-19272 & 9/8/05 & 80 & 2.6 & ND \\
\hline & & CNSB08-W-16317 & $10 / 12 / 05$ & 77 & 2.8 & ND \\
\hline & & CNSB08-W-19903 & $3 / 17 / 06$ & 91 & 2.7 & ND \\
\hline & & CNSB08-W-22500 & $9 / 21 / 06$ & 53 & 1.6 & ND \\
\hline & & CNSB08-W-16214 & $3 / 28 / 07$ & 64 & 2.0 & ND \\
\hline & & CNSB08-W-16229 & 9/26/07 & 68 & 1.8 & ND \\
\hline & & CNSB08-W-26004 & $3 / 12 / 08$ & 28 & 1.1 & ND \\
\hline & & CNSB08-W-26687 & $9 / 8 / 08$ & 22 & 1.2 & ND \\
\hline & & CBSB08-W-27163 & 10/8/09 & 29 & 1.2 & ND \\
\hline & & CNSB08-W-27200 & $9 / 20 / 10$ & 16 & $0.9 \mathrm{~J}$ & ND \\
\hline \multirow[t]{9}{*}{ SB09 } & $32-42$ & CNSB09-W-16193 & $8 / 26 / 04$ & ND & ND & ND \\
\hline & & CNSB09-W-19281 & 9/11/05 & ND & ND & ND \\
\hline & & CNSB09-W-16318 & $10 / 11 / 05$ & ND & ND & ND \\
\hline & & CNSBO9-W-19902 & $3 / 17 / 06$ & ND & ND & ND \\
\hline & & CNSB09-W-22502 & $9 / 25 / 06$ & ND & ND & ND \\
\hline & & CNSB09-W-16211 & $3 / 28 / 07$ & ND & ND & ND \\
\hline & & CNSB09-W-16231 & 9/26/07 & ND & ND & ND \\
\hline & & CNSB09-W-26033 & $3 / 20 / 08$ & ND & ND & ND \\
\hline & & CNSB09-W-26688 & $9 / 10 / 08$ & ND & ND & ND \\
\hline
\end{tabular}

a ND, not detected at an instrument detection limit of $0.1 \mu \mathrm{g} / \mathrm{L}$.

b Qalifier R indicates that the contaminant was present in the associated equipment rinsate.

c Data are for samples collected prior to implementation of the IM ISCR pilot test in November 2007. More recent results are in Table 3.4.

d Qalifier J indicates an estimated concentration below the method quantitation limit of $1.0 \mu \mathrm{g} / \mathrm{L}$.

e Qualifier B indicates that the contaminant was present in the associated method blank. 
TABLE 3.3 Field measurements for groundwater samples collected from the sitewide monitoring points at Centralia, August 2004 to September 2010.

\begin{tabular}{|c|c|c|c|c|c|c|c|c|c|}
\hline \multirow[b]{2}{*}{ Well } & \multirow[b]{2}{*}{$\begin{array}{l}\text { Screen } \\
\text { Interval } \\
\text { (ft BGL) }\end{array}$} & \multirow[b]{2}{*}{$\begin{array}{l}\text { Sample } \\
\text { Date }\end{array}$} & \multirow[b]{2}{*}{$\begin{array}{c}\text { Temperature } \\
\left({ }^{\circ} \mathrm{C}\right)\end{array}$} & \multirow[b]{2}{*}{$\mathrm{pH}$} & \multirow[b]{2}{*}{$\begin{array}{c}\text { Conductivity } \\
(\mu \mathrm{S} / \mathrm{cm})\end{array}$} & \multicolumn{3}{|c|}{ Concentration $(\mathrm{mg} / \mathrm{L})$} & \multirow[b]{2}{*}{$\begin{array}{l}\text { ORF } \\
(\mathrm{mV})\end{array}$} \\
\hline & & & & & & $\begin{array}{c}\text { Dissolved } \\
\text { Oxygen }\end{array}$ & $\begin{array}{l}\text { Carbon } \\
\text { Dioxide }\end{array}$ & Iron(II) & \\
\hline \multirow[t]{9}{*}{ MW01 } & $54.5-64.5$ & $8 / 24 / 04$ & 16.3 & 7.39 & 652 & 0.06 & 25 & 0.00 & 230 \\
\hline & & 9/10/05 & 16.3 & 7.26 & 599 & 6.31 & $-{ }^{a}$ & 0.00 & 104 \\
\hline & & $10 / 11 / 05$ & 16.4 & 6.45 & 634 & - & - & - & - \\
\hline & & $3 / 15 / 06$ & 14.3 & 7.56 & 621 & 9.33 & 30 & 0.04 & 297 \\
\hline & & 9/25/06 & 13.3 & 7.01 & 782 & 6.82 & 50 & 0.31 & 92 \\
\hline & & $3 / 29 / 07$ & 16.5 & 6.54 & 629 & 4.39 & - & 0.00 & 174 \\
\hline & & $9 / 26 / 07$ & 17.8 & 7.06 & 630 & 0.89 & 35 & 0.09 & 146 \\
\hline & & $3 / 19 / 08$ & 9.5 & 7.31 & 613 & 3.34 & - & - & 122 \\
\hline & & 9/9/08 & 13.9 & 7.28 & 595 & 5.18 & 20 & 0.03 & 28 \\
\hline \multirow[t]{7}{*}{$\mathrm{MWO}^{\mathrm{b}}$} & $49.5-59.5$ & $8 / 26 / 04$ & 14.4 & 7.31 & 729 & 0.16 & 20 & 0.12 & 235 \\
\hline & & 9/11/05 & 15.3 & 7.02 & 739 & 1.28 & - & - & - \\
\hline & & $10 / 12 / 05$ & 14.8 & 6.60 & 766 & - & - & - & - \\
\hline & & $3 / 16 / 06$ & 14.2 & 6.78 & 759 & 1.24 & - & 0.00 & 295 \\
\hline & & $9 / 26 / 06$ & 13.2 & 6.98 & 957 & 3.05 & 40 & 0.06 & 67 \\
\hline & & $3 / 26 / 07$ & 15.7 & 6.39 & 739 & 2.29 & 50 & - & 67 \\
\hline & & $9 / 26 / 07$ & 15.4 & 7.04 & 763 & 3.39 & 25 & 0.00 & 156 \\
\hline \multirow[t]{11}{*}{ MW03 } & $50.5-60.5$ & $8 / 24 / 04$ & 13.1 & 7.28 & 783 & 0.10 & 55 & 0.21 & 230 \\
\hline & & 9/10/05 & 15.1 & 7.05 & 715 & 10.42 & 65 & 0.00 & 142 \\
\hline & & $10 / 11 / 05$ & 16.3 & 6.46 & 765 & - & - & - & - \\
\hline & & $3 / 17 / 06$ & 13.8 & 6.75 & 753 & 9.39 & 77 & 0.00 & 290 \\
\hline & & $9 / 26 / 06$ & 13.2 & 6.92 & 960 & 11.57 & 45 & 0.08 & 251 \\
\hline & & $3 / 27 / 07$ & 15.3 & 6.40 & 774 & 7.73 & 25 & - & 268 \\
\hline & & $9 / 25 / 07$ & 14.3 & 6.97 & 738 & 8.44 & 30 & 0.00 & 162 \\
\hline & & $3 / 12 / 08$ & 14.6 & 7.12 & 777 & 7.90 & - & 3.13 & 88 \\
\hline & & $9 / 9 / 08$ & 14.9 & 7.13 & 763 & 9.60 & 110 & 0.12 & 66 \\
\hline & & $10 / 6 / 09$ & 13.8 & 7.08 & 770 & 9.66 & 95 & 0.03 & 216 \\
\hline & & 9/19/10 & 14.7 & 6.98 & 762 & 10.48 & - & 0.08 & 178 \\
\hline \multirow[t]{11}{*}{ MW04 } & $37.5-47.5$ & $8 / 24 / 04$ & 16.2 & 7.39 & 717 & 0.11 & 40 & 0.04 & 210 \\
\hline & & 9/11/05 & 15.4 & 7.18 & 665 & 8.43 & 60 & 0.00 & 226 \\
\hline & & $10 / 11 / 05$ & 14.4 & 7.14 & 811 & - & - & - & - \\
\hline & & $3 / 15 / 06$ & 13.5 & 7.78 & 675 & 6.82 & 55 & 0.06 & 283 \\
\hline & & 9/25/06 & - & 7.02 & 613 & 9.13 & 40 & 0.19 & 46 \\
\hline & & $3 / 28 / 07$ & 15.4 & 6.47 & 678 & 5.46 & - & 0.00 & 197 \\
\hline & & $9 / 24 / 07$ & 17.4 & 7.10 & 667 & 6.94 & 35 & 0.24 & 261 \\
\hline & & $3 / 19 / 08$ & 11.2 & 7.32 & 636 & 7.55 & - & - & 164 \\
\hline & & 9/9/08 & 14.2 & 7.14 & 648 & 8.68 & 100 & 0.00 & 72 \\
\hline & & $10 / 7 / 09$ & 13.9 & 7.17 & 671 & 8.64 & 100 & 0.02 & 183 \\
\hline & & 9/20/10 & 16.2 & 7.18 & 572 & 8.91 & - & 0.10 & 164 \\
\hline
\end{tabular}


TABLE 3.3 (Cont.)

\begin{tabular}{|c|c|c|c|c|c|c|c|c|c|}
\hline \multirow[b]{2}{*}{ Well } & \multirow{2}{*}{$\begin{array}{l}\text { Screen } \\
\text { Interval } \\
\text { (ft BGL) }\end{array}$} & \multirow[b]{2}{*}{$\begin{array}{l}\text { Sample } \\
\text { Date }\end{array}$} & \multirow[b]{2}{*}{$\begin{array}{c}\text { Temperature } \\
\left({ }^{\circ} \mathrm{C}\right)\end{array}$} & \multirow[b]{2}{*}{$\mathrm{pH}$} & \multirow[b]{2}{*}{$\begin{array}{l}\text { Conductivity } \\
(\mu \mathrm{S} / \mathrm{cm})\end{array}$} & \multicolumn{3}{|c|}{ Concentration (mg/L) } & \multirow[b]{2}{*}{$\begin{array}{l}\text { ORP } \\
(\mathrm{mV})\end{array}$} \\
\hline & & & & & & $\begin{array}{c}\text { Dissolved } \\
\text { Oxygen }\end{array}$ & $\begin{array}{l}\text { Carbon } \\
\text { Dioxide }\end{array}$ & Iron(II) & \\
\hline \multirow[t]{11}{*}{ MW05 } & $34.5-44.5$ & $8 / 25 / 04$ & 14.3 & 7.14 & 613 & 0.08 & 25 & 0.06 & 215 \\
\hline & & 9/10/05 & 14.2 & 6.80 & 620 & 1.40 & 110 & 0.00 & 160 \\
\hline & & $10 / 11 / 05$ & 14.8 & 6.35 & 610 & - & - & - & - \\
\hline & & 3/15/06 & 14.3 & 6.90 & 701 & 0.90 & 30 & 0.06 & 156 \\
\hline & & $9 / 25 / 06$ & 13.6 & 6.95 & 768 & 0.09 & 50 & 0.02 & 55 \\
\hline & & $3 / 28 / 07$ & 14.4 & 6.44 & 573 & 4.53 & 35 & 0.00 & 295 \\
\hline & & $9 / 24 / 07$ & 15.8 & 7.06 & 368 & 3.09 & 45 & 0.00 & 182 \\
\hline & & $3 / 19 / 08$ & 12.9 & 7.42 & 642 & 5.42 & - & - & 177 \\
\hline & & 9/10/08 & 13.9 & 7.11 & 663 & 7.14 & 95 & 0.00 & 130 \\
\hline & & $10 / 7 / 09$ & 14.2 & 7.11 & 672 & 7.05 & 90 & 0.00 & 194 \\
\hline & & 9/20/10 & 17.2 & 7.18 & 675 & 6.07 & - & 0.01 & 183 \\
\hline \multirow[t]{11}{*}{ MW06 } & $46.5-56.5$ & $8 / 25 / 04$ & 15.9 & 7.50 & 637 & 0.05 & 15 & 0.00 & 215 \\
\hline & & 9/10/05 & 14.6 & 7.23 & 659 & 0.04 & 60 & 0.00 & 41 \\
\hline & & $10 / 11 / 05$ & 15.8 & 6.99 & 638 & - & - & - & - \\
\hline & & $3 / 15 / 06$ & 14.1 & 7.38 & 630 & 9.87 & 35 & 0.02 & 263 \\
\hline & & $9 / 27 / 06$ & 13.1 & 6.16 & 652 & 0.05 & 45 & 1.12 & 63 \\
\hline & & $3 / 27 / 07$ & 19.0 & 6.42 & 466 & 0.11 & 20 & 0.00 & 13 \\
\hline & & $9 / 24 / 07$ & 16.8 & 7.11 & 463 & 8.00 & 25 & 0.41 & 191 \\
\hline & & $3 / 19 / 08$ & 14.1 & 7.01 & 552 & 7.00 & - & - & 172 \\
\hline & & 9/9/08 & 14.4 & 7.20 & 437 & 0.36 & 105 & 0.07 & -96 \\
\hline & & 10/6/09 & 13.5 & 6.69 & 255 & 0.61 & 110 & 0.06 & -72 \\
\hline & & $9 / 20 / 10$ & 15.6 & 6.97 & 369 & 2.48 & - & 0.04 & 86 \\
\hline \multirow[t]{8}{*}{ MW07 } & $45-55$ & $3 / 14 / 06$ & 14.7 & 6.61 & 709 & 0.34 & - & 0.03 & 143 \\
\hline & & 9/26/06 & 13.1 & 7.23 & 642 & 2.91 & 50 & 0.00 & - \\
\hline & & $3 / 26 / 07$ & 15.8 & 6.50 & 642 & 1.87 & 30 & 0.00 & 261 \\
\hline & & $9 / 24 / 07$ & 19.0 & 7.18 & 609 & 9.05 & 60 & 0.18 & 190 \\
\hline & & 3/19/08 & 12.5 & 7.29 & 647 & 2.70 & - & - & 215 \\
\hline & & 9/9/08 & 15.6 & 7.10 & 629 & 1.41 & 68 & 0.00 & 16 \\
\hline & & 10/6/09 & 13.9 & 7.19 & 618 & 1.42 & 70 & 0.00 & 53 \\
\hline & & $9 / 20 / 10$ & 16.6 & 7.22 & 622 & 2.93 & - & 0.00 & 132 \\
\hline \multirow[t]{6}{*}{ MW08 } & $38-53$ & $3 / 14 / 06$ & 13.5 & 6.35 & 854 & 5.32 & - & 0.00 & 145 \\
\hline & & $9 / 26 / 06$ & 13.3 & 6.75 & 1095 & 0.16 & 50 & 0.18 & 37 \\
\hline & & $3 / 27 / 07$ & 15.8 & 6.31 & 874 & 1.49 & 30 & 0.21 & 237 \\
\hline & & $9 / 25 / 07$ & 15.8 & 6.92 & 627 & 1.42 & 45 & 0.14 & 219 \\
\hline & & 3/20/08 & 13.5 & 7.19 & 869 & 2.11 & - & - & 185 \\
\hline & & 9/10/08 & 16.3 & 7.03 & 864 & 1.17 & 100 & 0.03 & 117 \\
\hline \multirow[t]{8}{*}{ MW09 } & $25-35$ & $3 / 15 / 06$ & 17.7 & 7.33 & 664 & 0.95 & 55 & 0.09 & 214 \\
\hline & & $9 / 25 / 06$ & 12.8 & 6.87 & 859 & 1.59 & 45 & 0.18 & 90 \\
\hline & & $3 / 27 / 07$ & 14.9 & 6.35 & 689 & 4.10 & 30 & 0.69 & 152 \\
\hline & & $9 / 24 / 07$ & 16.6 & 6.94 & 1999 & 3.86 & 55 & 0.14 & 186 \\
\hline & & $3 / 20 / 08$ & 13.5 & 7.17 & 720 & 4.70 & - & - & 173 \\
\hline & & 9/10/08 & 14.7 & 7.02 & 706 & 3.68 & 110 & 0.07 & 120 \\
\hline & & $10 / 6 / 09$ & 13.2 & 7.00 & 715 & 3.73 & 110 & 0.08 & 148 \\
\hline & & 9/19/10 & 14.6 & 6.99 & 711 & 3.60 & - & 0.09 & 159 \\
\hline
\end{tabular}


TABLE 3.3 (Cont.)

\begin{tabular}{|c|c|c|c|c|c|c|c|c|c|}
\hline \multirow[b]{2}{*}{ Well } & \multirow{2}{*}{$\begin{array}{l}\text { Screen } \\
\text { Interval } \\
\text { (ft BGL) }\end{array}$} & \multirow[b]{2}{*}{$\begin{array}{l}\text { Sample } \\
\text { Date }\end{array}$} & \multirow[b]{2}{*}{$\begin{array}{c}\text { Temperature } \\
\left({ }^{\circ} \mathrm{C}\right)\end{array}$} & \multirow[b]{2}{*}{$\mathrm{pH}$} & \multirow[b]{2}{*}{$\begin{array}{c}\text { Conductivity } \\
(\mu \mathrm{S} / \mathrm{cm})\end{array}$} & \multicolumn{3}{|c|}{ Concentration $(\mathrm{mg} / \mathrm{L})$} & \multirow[b]{2}{*}{$\begin{array}{l}\text { ORP } \\
(\mathrm{mV})\end{array}$} \\
\hline & & & & & & $\begin{array}{c}\text { Dissolved } \\
\text { Oxygen }\end{array}$ & $\begin{array}{l}\text { Carbon } \\
\text { Dioxide }\end{array}$ & Iron(II) & \\
\hline \multirow[t]{8}{*}{ MW10 } & $30-45$ & $3 / 14 / 06$ & 14.8 & 6.60 & 834 & 6.42 & 65 & 0.00 & 166 \\
\hline & & 9/26/06 & 13.6 & 6.87 & 1058 & 6.94 & 50 & 0.50 & 51 \\
\hline & & $3 / 28 / 07$ & 17.0 & 6.36 & 834 & 5.09 & 35 & 0.00 & 270 \\
\hline & & 9/25/07 & 15.8 & 6.94 & 827 & 6.64 & 35 & 0.21 & 199 \\
\hline & & $3 / 20 / 08$ & 10.9 & 7.18 & 898 & 6.12 & - & - & 187 \\
\hline & & 9/9/08 & 14.8 & 7.05 & 879 & 7.18 & 100 & 0.06 & 94 \\
\hline & & $10 / 6 / 09$ & 13.7 & 7.04 & 883 & 6.67 & 95 & 0.08 & 201 \\
\hline & & 9/19/10 & 15.1 & 6.95 & 882 & 6.76 & - & 0.00 & 186 \\
\hline \multirow[t]{11}{*}{ SB01 } & $40-50$ & $8 / 26 / 04$ & 26.0 & 7.46 & 699 & 5.21 & 30 & 0.00 & 210 \\
\hline & & 9/9/05 & 25.0 & 7.11 & 674 & 6.25 & 95 & 0.00 & 140 \\
\hline & & $10 / 12 / 05$ & 13.8 & 7.23 & 686 & - & - & - & - \\
\hline & & $3 / 17 / 06$ & 12.4 & 7.30 & 692 & 5.98 & 55 & 0.00 & 185 \\
\hline & & 9/27/06 & 14.4 & 7.03 & 832 & 6.54 & 40 & 0.52 & 198 \\
\hline & & $3 / 27 / 07$ & 18.0 & 6.37 & 659 & 3.81 & 25 & 0.23 & 173 \\
\hline & & 9/27/07 & 13.5 & 7.24 & 720 & 6.55 & 45 & 1.04 & 143 \\
\hline & & $3 / 20 / 08$ & 15.6 & 7.29 & 783 & 8.02 & - & - & 182 \\
\hline & & 9/10/08 & 16.5 & 7.10 & 676 & 2.89 & 100 & 0.17 & 100 \\
\hline & & $10 / 7 / 09$ & 14.8 & 7.11 & 761 & 7.69 & 105 & 0.07 & 215 \\
\hline & & 9/20/10 & 17.1 & 7.24 & 679 & 7.10 & - & 0.00 & 163 \\
\hline \multirow[t]{11}{*}{ SB04 } & $51-61$ & $8 / 26 / 04$ & 17.9 & 7.14 & 765 & 3.78 & 55 & 0.37 & 230 \\
\hline & & 9/9/05 & 16.0 & 7.09 & 708 & 8.67 & 100 & - & 206 \\
\hline & & $10 / 12 / 05$ & 13.9 & 7.17 & 813 & - & - & - & - \\
\hline & & $3 / 16 / 06$ & 13.0 & 7.57 & 799 & 5.96 & 30 & - & 276 \\
\hline & & 9/25/06 & 14.9 & 7.16 & 791 & 9.32 & 70 & 1.18 & 64 \\
\hline & & $3 / 28 / 07$ & 16.2 & 6.45 & 850 & 6.18 & - & 0.23 & 266 \\
\hline & & $9 / 26 / 07$ & 19.8 & 7.03 & 760 & 6.61 & 30 & 0.00 & 202 \\
\hline & & $3 / 12 / 08$ & 15.5 & 7.04 & 819 & 6.16 & - & 0.09 & 154 \\
\hline & & 9/9/08 & 16.5 & 7.11 & 802 & 6.48 & 100 & 0.02 & 70 \\
\hline & & $10 / 8 / 09$ & 12.2 & 7.11 & 797 & 7.43 & 95 & 0.09 & 238 \\
\hline & & 9/20/10 & 22.3 & 7.04 & 806 & 6.98 & - & 0.06 & 143 \\
\hline \multirow[t]{11}{*}{ SB05 } & $32-42$ & $8 / 26 / 04$ & 15.7 & 7.25 & 761 & - & 25 & 0.06 & 220 \\
\hline & & 9/9/05 & 16.9 & 6.98 & 687 & 7.58 & 100 & - & - \\
\hline & & $10 / 12 / 05$ & 14.0 & 7.00 & 728 & - & - & - & - \\
\hline & & $3 / 17 / 06$ & 13.3 & 7.67 & 718 & 4.80 & 40 & 0.18 & 253 \\
\hline & & 9/27/06 & 13.7 & 6.58 & 763 & 4.70 & 50 & 0.25 & 78 \\
\hline & & $3 / 28 / 07$ & 16.7 & 4.03 & 1100 & 2.58 & 35 & 0.07 & 296 \\
\hline & & 9/26/07 & 15.1 & 6.98 & 810 & 4.10 & 30 & 0.50 & 221 \\
\hline & & $3 / 20 / 08$ & 14.5 & 7.11 & 870 & 5.56 & - & - & 206 \\
\hline & & 9/9/08 & 13.7 & 6.79 & 890 & 7.60 & 90 & 0.09 & 56 \\
\hline & & $10 / 8 / 09$ & 12.7 & 7.09 & 874 & 6.63 & 100 & 0.08 & 209 \\
\hline & & $9 / 21 / 10$ & 14.4 & 7.18 & 862 & 7.69 & - & 0.54 & 60 \\
\hline \multirow[t]{8}{*}{ SB07R } & $45-60$ & $3 / 15 / 06$ & 16.8 & 7.24 & 685 & 7.41 & 60 & 0.08 & 83 \\
\hline & & 9/26/06 & 13.2 & 6.89 & 842 & 6.17 & 55 & 0.26 & 67 \\
\hline & & $3 / 26 / 07$ & 19.0 & 6.38 & 668 & 5.08 & 40 & 0.07 & 237 \\
\hline & & $9 / 25 / 07$ & 17.4 & 7.06 & 642 & 6.30 & 35 & 0.11 & 170 \\
\hline & & $3 / 12 / 08$ & 17.3 & 7.18 & 639 & 5.33 & - & 0.00 & 108 \\
\hline & & 9/9/08 & 14.1 & 7.06 & 631 & 5.08 & 100 & 0.07 & 55 \\
\hline & & $10 / 7 / 09$ & 13.3 & 7.11 & 629 & 6.67 & 110 & 0.10 & 224 \\
\hline & & 9/20/10 & 15.5 & 7.04 & 648 & 5.87 & - & 0.13 & 161 \\
\hline
\end{tabular}


TABLE 3.3 (Cont.)

\begin{tabular}{|c|c|c|c|c|c|c|c|c|c|}
\hline \multirow[b]{2}{*}{ Well } & \multirow{2}{*}{$\begin{array}{l}\text { Screen } \\
\text { Interval } \\
\text { (ft BGL) }\end{array}$} & \multirow[b]{2}{*}{$\begin{array}{l}\text { Sample } \\
\text { Date }\end{array}$} & \multirow[b]{2}{*}{$\begin{array}{c}\text { Temperature } \\
\left({ }^{\circ} \mathrm{C}\right)\end{array}$} & \multirow[b]{2}{*}{$\mathrm{pH}$} & \multirow[b]{2}{*}{$\begin{array}{l}\text { Conductivity } \\
(\mu S / \mathrm{cm})\end{array}$} & \multicolumn{3}{|c|}{ Concentration (mg/L) } & \multirow[b]{2}{*}{$\begin{array}{l}\text { ORF } \\
(\mathrm{mV}\end{array}$} \\
\hline & & & & & & $\begin{array}{l}\text { Dissolved } \\
\text { Oxygen }\end{array}$ & $\begin{array}{l}\text { Carbon } \\
\text { Dioxide }\end{array}$ & Iron(II) & \\
\hline \multirow[t]{11}{*}{ SB08 } & $52-62$ & 8/26/04 & 19.5 & 7.31 & 635 & 0.16 & 20 & 0.53 & 235 \\
\hline & & 9/8/05 & 21.2 & 7.27 & 598 & 3.21 & 75 & 0.00 & 111 \\
\hline & & $10 / 12 / 05$ & 13.9 & 7.15 & 630 & - & - & - & - \\
\hline & & $3 / 17 / 06$ & 12.9 & 7.14 & 645 & 3.40 & 40 & 0.00 & 246 \\
\hline & & $9 / 21 / 06$ & 14.1 & 6.96 & 809 & 4.53 & 40 & 0.00 & 37 \\
\hline & & $3 / 28 / 07$ & 15.8 & 6.53 & 645 & 3.57 & 35 & 0.24 & 208 \\
\hline & & $9 / 26 / 07$ & 17.4 & 7.11 & 617 & 4.56 & 40 & 0.77 & 156 \\
\hline & & $3 / 12 / 08$ & 17.1 & 7.17 & 642 & 3.63 & - & 0.14 & 102 \\
\hline & & 9/8/08 & 13.6 & 7.14 & 626 & 2.70 & 90 & 0.00 & 230 \\
\hline & & $10 / 8 / 09$ & 12.3 & 7.22 & 617 & 4.43 & 95 & 0.00 & 221 \\
\hline & & $9 / 20 / 10$ & 15.2 & 7.12 & 616 & 3.73 & - & 0.05 & 166 \\
\hline \multirow[t]{9}{*}{ SB09 } & $32-42$ & $8 / 26 / 04$ & 30.9 & 7.09 & 910 & 0.26 & 75 & 0.00 & 185 \\
\hline & & 9/11/05 & 14.6 & 6.71 & 877 & 0.13 & 225 & 0.00 & - \\
\hline & & $10 / 11 / 05$ & 13.9 & 6.85 & 910 & - & - & - & - \\
\hline & & $3 / 17 / 06$ & 11.7 & 7.03 & 969 & 1.53 & 99 & 0.00 & 206 \\
\hline & & $9 / 25 / 06$ & 14.2 & 7.00 & 976 & 0.29 & 70 & 0.38 & 86 \\
\hline & & $3 / 28 / 07$ & 14.3 & 6.32 & 957 & 0.89 & 40 & 0.09 & 236 \\
\hline & & 9/26/07 & 15.2 & 6.77 & 969 & 1.53 & 45 & 0.12 & 199 \\
\hline & & 3/20/08 & 10.1 & 6.94 & 1000 & 1.57 & - & - & 221 \\
\hline & & 9/10/08 & 18.4 & 6.87 & 977 & 0.56 & 160 & 0.11 & 109 \\
\hline
\end{tabular}

a No measurement obtained.

b Data are for samples collected prior to implementation of the IM ISCR pilot test in November 2007. More recent data are in Table 3.5. 
TABLE 3.4 Analytical results from the AGEM Laboratory for volatile organic compounds in groundwater samples collected from the IM pilot test monitoring points at Centralia, September 2008 to September 2010.

\begin{tabular}{|c|c|c|c|c|c|c|}
\hline \multirow[b]{2}{*}{ Well } & \multirow[b]{2}{*}{$\begin{array}{l}\text { Screen } \\
\text { Interval } \\
\text { (ft BGL) }\end{array}$} & \multirow[b]{2}{*}{ Sample } & \multirow[b]{2}{*}{$\begin{array}{l}\text { Sample } \\
\text { Date }\end{array}$} & \multicolumn{3}{|c|}{ Concentration $(\mu \mathrm{g} / \mathrm{L})$} \\
\hline & & & & $\begin{array}{l}\text { Carbon } \\
\text { Tetrachloride }\end{array}$ & Chloroform & $\begin{array}{c}\text { Methylene } \\
\text { Chloride }\end{array}$ \\
\hline \multirow[t]{5}{*}{$\mathrm{MWO2}^{\mathrm{a}}$} & $49.5-59.5$ & CNMW02-W-26674 & 9/8/08 & 18 & 57 & 11 \\
\hline & & CNMW02-W-27140 & $4 / 22 / 09$ & $N D^{b}$ & ND & 1.8 \\
\hline & & CNMW02-W-27150 & 10/8/09 & ND & ND & ND \\
\hline & & CNMW02-W-27179 & $4 / 5 / 10$ & ND & ND & ND \\
\hline & & CNMW02-W-27187 & $9 / 20 / 10$ & 1.7 & ND & ND \\
\hline \multirow[t]{5}{*}{ PMP1 } & $50-60$ & CNPMP1-W-26689 & 9/9/08 & 136 & 30 & ND \\
\hline & & CNPMP1-W-27141 & $4 / 22 / 09$ & 102 & 21 & ND \\
\hline & & CNPMP1-W-27165 & $10 / 7 / 09$ & 167 & 20 & ND \\
\hline & & CNPMP1-W-27180 & $4 / 5 / 10$ & 91 & 15 & ND \\
\hline & & CNPMP1-W-27202 & $9 / 21 / 10$ & 103 & 11 & ND \\
\hline \multirow[t]{5}{*}{ PMP2 } & $50-60$ & CNPMP2-W-26690 & 9/9/08 & 1854 & 318 & 5.6 \\
\hline & & CNPMP2-W-27142 & $4 / 22 / 09$ & 1398 & 299 & $-c$ \\
\hline & & CNPMP2-W-27166 & $10 / 7 / 09$ & 1384 & 272 & 6.6 \\
\hline & & CNPMP2-W-27181 & $4 / 5 / 10$ & 991 & 182 & 5.1 \\
\hline & & CNPMP2-W-27203 & $9 / 21 / 10$ & 117 & 55 & 2.3 \\
\hline \multirow[t]{5}{*}{ PMP3 } & $50-60$ & CNPMP3-W-26691 & 9/9/08 & 21 & 57 & 6.2 \\
\hline & & CNPMP3-W-27143 & $4 / 22 / 09$ & 3.2 & 5.8 & ND \\
\hline & & CNPMP3-W-27167 & $10 / 7 / 09$ & $0.5 \mathrm{~J}^{\mathrm{d}}$ & 3.9 & ND \\
\hline & & CNPMP3-W-27182 & $4 / 5 / 10$ & ND & ND & ND \\
\hline & & CNPMP3-W-27204 & $9 / 21 / 10$ & ND & ND & ND \\
\hline \multirow[t]{3}{*}{ PMP4 } & $48.75-58.75$ & CNPMP4-W-26692 & 9/9/08 & 49 & 4.2 & ND \\
\hline & & CNPMP4-W-27168 & $10 / 6 / 09$ & 39 & 2.9 & ND \\
\hline & & CNPMP4-W-27205 & $9 / 21 / 10$ & 28 & 1.8 & ND \\
\hline \multirow[t]{3}{*}{ PMP5 } & $50-60$ & CNPMP5-W-26693 & 9/10/08 & 418 & 46 & 1.6 \\
\hline & & CNPMP5-W-27169 & $10 / 8 / 09$ & 728 & 43 & 1.2 \\
\hline & & CNPMP5-W-27206 & $9 / 20 / 10$ & 779 & 35 & $0.9 \mathrm{~J}$ \\
\hline \multirow[t]{3}{*}{ PMP6 } & $50-60$ & CNPMP6-W-26694 & 9/8/08 & 110 & 7.8 & ND \\
\hline & & CNPMP6-W-27170 & 10/6/09 & 199 & 12 & ND \\
\hline & & CNPMP6-W-27207 & $9 / 21 / 10$ & 143 & 9.6 & ND \\
\hline \multirow[t]{3}{*}{ PMP7 } & $50-60$ & CNPMP7-W-26695 & 9/9/08 & 119 & 13 & ND \\
\hline & & CNPMP7-W-27171 & $10 / 6 / 09$ & 84 & 23 & 1.8 \\
\hline & & CNPMP7-W-27208 & $9 / 21 / 10$ & 98 & 37 & 4.0 \\
\hline
\end{tabular}


TABLE 3.4 (Cont.)

\begin{tabular}{|c|c|c|c|c|c|c|}
\hline \multirow[b]{2}{*}{ Well } & \multirow{2}{*}{$\begin{array}{l}\text { Screen } \\
\text { Interval } \\
\text { (ft BGL) }\end{array}$} & \multirow[b]{2}{*}{ Sample } & \multirow[b]{2}{*}{$\begin{array}{l}\text { Sample } \\
\text { Date }\end{array}$} & \multicolumn{3}{|c|}{ Concentration $(\mu \mathrm{g} / \mathrm{L})$} \\
\hline & & & & $\begin{array}{c}\text { Carbon } \\
\text { Tetrachloride }\end{array}$ & Chloroform & $\begin{array}{l}\text { Methylene } \\
\text { Chloride }\end{array}$ \\
\hline \multirow[t]{5}{*}{ PMP8 } & $50-60$ & CNPMP8-W-26696 & 9/9/08 & 72 & 125 & 3.4 \\
\hline & & CNPMP8-W-27144 & $4 / 22 / 09$ & 3.2 & 5.6 & 1.9 \\
\hline & & CNPMP8-W-27172 & $10 / 7 / 09$ & 16 & 21 & 1.8 \\
\hline & & CNPMP8-W-27183 & $4 / 5 / 10$ & $0.4 \mathrm{~J}$ & $0.7 \mathrm{~J}$ & ND \\
\hline & & CNPMP8-W-27209 & $9 / 21 / 10$ & $0.7 \mathrm{~J}$ & ND & ND \\
\hline \multirow[t]{3}{*}{ PMP9 } & $50-60$ & CNPMP9-W-26697 & 9/9/08 & 7.6 & $0.4 \mathrm{~J}$ & ND \\
\hline & & CNPMP9-W-27173 & $10 / 7 / 09$ & 29 & $0.5 \mathrm{~J}$ & ND \\
\hline & & CNPMP9-W-27210 & $9 / 21 / 10$ & 24 & $0.2 \mathrm{~J}$ & ND \\
\hline
\end{tabular}

a Data are for samples collected after implementation of the IM ISCR pilot test in November 2007. Earlier data are in Table 3.2.

b ND, not detected at an instrument detection limit of $0.1 \mu \mathrm{g} / \mathrm{L}$.

c No analysis.

d Qualifier J indicates an estimated concentration below the method quantitation limit of $1.0 \mu \mathrm{g} / \mathrm{L}$. 
TABLE 3.5 Field measurements for groundwater samples collected from the IM pilot test monitoring points at Centralia, September 2008 to September 2010.

\begin{tabular}{|c|c|c|c|c|c|c|c|c|c|}
\hline \multirow[b]{2}{*}{ Well } & \multirow[b]{2}{*}{$\begin{array}{l}\text { Screen } \\
\text { Interval } \\
\text { (ft BGL) }\end{array}$} & \multirow[b]{2}{*}{$\begin{array}{l}\text { Sample } \\
\text { Date }\end{array}$} & \multirow[b]{2}{*}{$\begin{array}{c}\text { Temperature } \\
\left({ }^{\circ} \mathrm{C}\right)\end{array}$} & \multirow[b]{2}{*}{$\mathrm{pH}$} & \multirow[b]{2}{*}{$\begin{array}{l}\text { Conductivity } \\
(\mu \mathrm{S} / \mathrm{cm})\end{array}$} & \multicolumn{3}{|c|}{ Concentration $(\mathrm{mg} / \mathrm{L})$} & \multirow[b]{2}{*}{$\begin{array}{l}\text { ORP } \\
(\mathrm{mV})\end{array}$} \\
\hline & & & & & & $\begin{array}{l}\text { Dissolved } \\
\text { Oxygen }\end{array}$ & $\begin{array}{l}\text { Carbon } \\
\text { Dioxide }\end{array}$ & Iron(II) & \\
\hline \multirow[t]{5}{*}{$\mathrm{MWO2}^{\mathrm{a}}$} & $49.5-59.5$ & 9/8/08 & 13.1 & 6.12 & 6821 & 0.40 & 50 & $3.30^{\mathrm{b}}$ & -74 \\
\hline & & $4 / 22 / 09$ & 14.8 & 6.71 & 2943 & 0.60 & 110 & 2.70 & -131 \\
\hline & & $10 / 8 / 09$ & 12.7 & 6.98 & 1829 & 0.44 & 50 & 3.06 & -138 \\
\hline & & $4 / 5 / 10$ & 15.0 & 8.79 & 1675 & 0.08 & 115 & 2.36 & -72 \\
\hline & & 9/20/10 & 15.7 & 6.98 & 1608 & 0.01 & - & $3.30^{b}$ & -139 \\
\hline \multirow{5}{*}{ PMP1 } & $50-60$ & 9/9/08 & 14.4 & 5.54 & 700 & 1.37 & 115 & 0.23 & 40 \\
\hline & & $4 / 22 / 09$ & 15.1 & 6.97 & 667 & 3.62 & 115 & 0.60 & -79 \\
\hline & & $10 / 7 / 09$ & 13.8 & 7.30 & 623 & 0.56 & 110 & 0.33 & -34 \\
\hline & & $4 / 5 / 10$ & 15.0 & 7.13 & 545 & 0.24 & 110 & 0.00 & 53 \\
\hline & & 9/21/10 & 15.8 & 6.83 & 617 & 0.53 & - & 0.67 & 34 \\
\hline \multirow[t]{5}{*}{ PMP2 } & $50-60$ & 9/9/08 & 14.4 & 7.09 & 997 & 0.05 & 180 & 1.68 & -41 \\
\hline & & $4 / 22 / 09$ & 15.0 & 6.91 & 829 & 3.57 & 150 & 1.36 & -101 \\
\hline & & $10 / 7 / 09$ & 13.9 & 7.65 & 775 & 0.19 & 160 & 1.53 & -89 \\
\hline & & $4 / 5 / 10$ & 13.6 & 7.05 & 667 & 0.22 & 140 & 1.87 & -93 \\
\hline & & 9/21/10 & 15.8 & 6.82 & 747 & 0.21 & - & 3.06 & -90 \\
\hline \multirow[t]{5}{*}{ PMP3 } & $50-60$ & 9/9/08 & 14.5 & 6.98 & 1301 & 0.03 & 150 & $3.30^{\mathrm{b}}$ & -150 \\
\hline & & $4 / 22 / 09$ & 14.3 & 7.13 & 506 & 2.64 & 130 & 2.51 & -114 \\
\hline & & $10 / 7 / 09$ & 14.0 & 8.06 & 472 & 0.17 & 140 & 0.37 & -129 \\
\hline & & $4 / 5 / 10$ & 13.3 & 7.59 & 433 & 0.16 & 140 & 0.24 & -175 \\
\hline & & $9 / 21 / 10$ & 16.1 & 7.28 & 492 & 2.02 & - & 1.18 & -138 \\
\hline \multirow[t]{3}{*}{ PMP4 } & $48.75-58.75$ & 9/9/08 & 14.3 & 4.97 & 738 & 4.87 & 100 & 0.49 & 134 \\
\hline & & 10/6/09 & 13.2 & 6.46 & 705 & 2.20 & 110 & 0.08 & 43 \\
\hline & & $9 / 21 / 10$ & 15.5 & 7.15 & 747 & 5.66 & - & 0.25 & 36 \\
\hline \multirow[t]{3}{*}{ PMP5 } & $50-60$ & 9/10/08 & 16.9 & 7.20 & 875 & 2.51 & 105 & 0.18 & 117 \\
\hline & & $10 / 8 / 09$ & 10.7 & 7.10 & 839 & 3.18 & 100 & 0.00 & 43 \\
\hline & & 9/20/10 & 20.0 & 7.05 & 904 & 3.35 & - & 0.12 & 92 \\
\hline \multirow[t]{3}{*}{ PMP6 } & $50-60$ & 9/8/08 & 13.2 & 6.87 & 787 & 3.32 & 75 & 0.09 & 173 \\
\hline & & $10 / 6 / 09$ & 13.5 & 6.80 & 692 & 2.30 & 80 & 0.07 & 159 \\
\hline & & $9 / 21 / 10$ & 15.5 & 7.22 & 777 & 1.90 & - & 0.59 & 91 \\
\hline \multirow[t]{3}{*}{ PMP7 } & $50-60$ & 9/9/08 & 14.2 & 6.30 & 807 & 2.18 & 70 & 0.18 & 15 \\
\hline & & $10 / 6 / 09$ & 13.4 & 6.74 & 655 & 0.46 & 70 & 0.12 & -13 \\
\hline & & $9 / 21 / 10$ & 15.2 & 7.23 & 664 & 0.20 & - & 0.07 & -38 \\
\hline \multirow[t]{5}{*}{ PMP8 } & $50-60$ & 9/9/08 & 14.4 & 7.05 & 1388 & 0.03 & 60 & 2.72 & -129 \\
\hline & & $4 / 22 / 09$ & 15.2 & 7.30 & 776 & 1.74 & 150 & 2.03 & -139 \\
\hline & & $10 / 7 / 09$ & 13.9 & 7.69 & 688 & 0.81 & 120 & 0.27 & -155 \\
\hline & & $4 / 5 / 10$ & 13.3 & 7.46 & 555 & 0.19 & 145 & 0.92 & -156 \\
\hline & & 9/21/10 & 14.8 & 7.44 & 592 & 2.00 & - & 1.66 & -138 \\
\hline
\end{tabular}


Monitoring at Centralia, Kansas, in 2010

Version 00, 02/28/11

TABLE 3.5 (Cont.)

\begin{tabular}{|c|c|c|c|c|c|c|c|c|c|}
\hline \multirow[b]{2}{*}{ Well } & \multirow{2}{*}{$\begin{array}{l}\text { Screen } \\
\text { Interval } \\
\text { (ft BGL) }\end{array}$} & \multirow[b]{2}{*}{$\begin{array}{l}\text { Sample } \\
\text { Date }\end{array}$} & \multirow[b]{2}{*}{$\begin{array}{c}\text { Temperature } \\
\left({ }^{\circ} \mathrm{C}\right)\end{array}$} & \multirow[b]{2}{*}{$\mathrm{pH}$} & \multirow[b]{2}{*}{$\begin{array}{l}\text { Conductivity } \\
(\mu S / \mathrm{cm})\end{array}$} & \multicolumn{3}{|c|}{ Concentration (mg/L) } & \multirow[b]{2}{*}{$\begin{array}{l}\text { ORP } \\
(\mathrm{mV})\end{array}$} \\
\hline & & & & & & $\begin{array}{l}\text { Dissolved } \\
\text { Oxygen }\end{array}$ & $\begin{array}{l}\text { Carbon } \\
\text { Dioxide }\end{array}$ & Iron(II) & \\
\hline \multirow[t]{3}{*}{ PMP9 } & $50-60$ & 9/9/08 & 14.0 & 6.36 & 606 & 7.78 & 120 & 0.10 & 45 \\
\hline & & 10/7/09 & 13.7 & 7.50 & 568 & 5.82 & 125 & 0.06 & -1 \\
\hline & & 9/21/10 & 15.2 & 7.26 & 605 & 6.67 & - & 0.15 & 44 \\
\hline
\end{tabular}

a Data are for samples collected after implementation of the IM ISCR pilot test in November 2007. Earlier results are in Table 3.3.

b Maximum reading from instrument. 


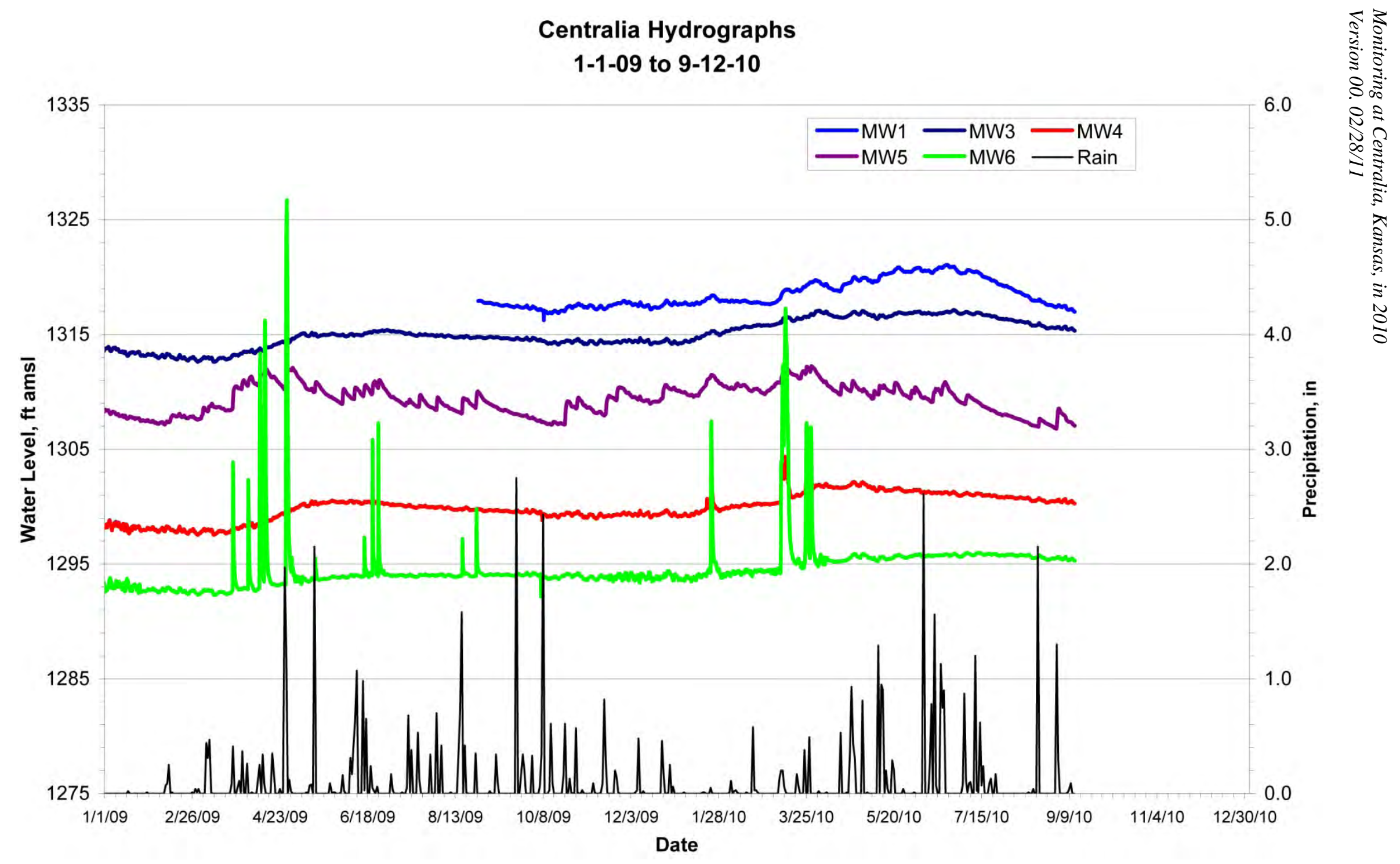

FIGURE 3.1 Hydrographs summarizing results of long-term water level monitoring at Centralia, January 2009 to September 2010. 


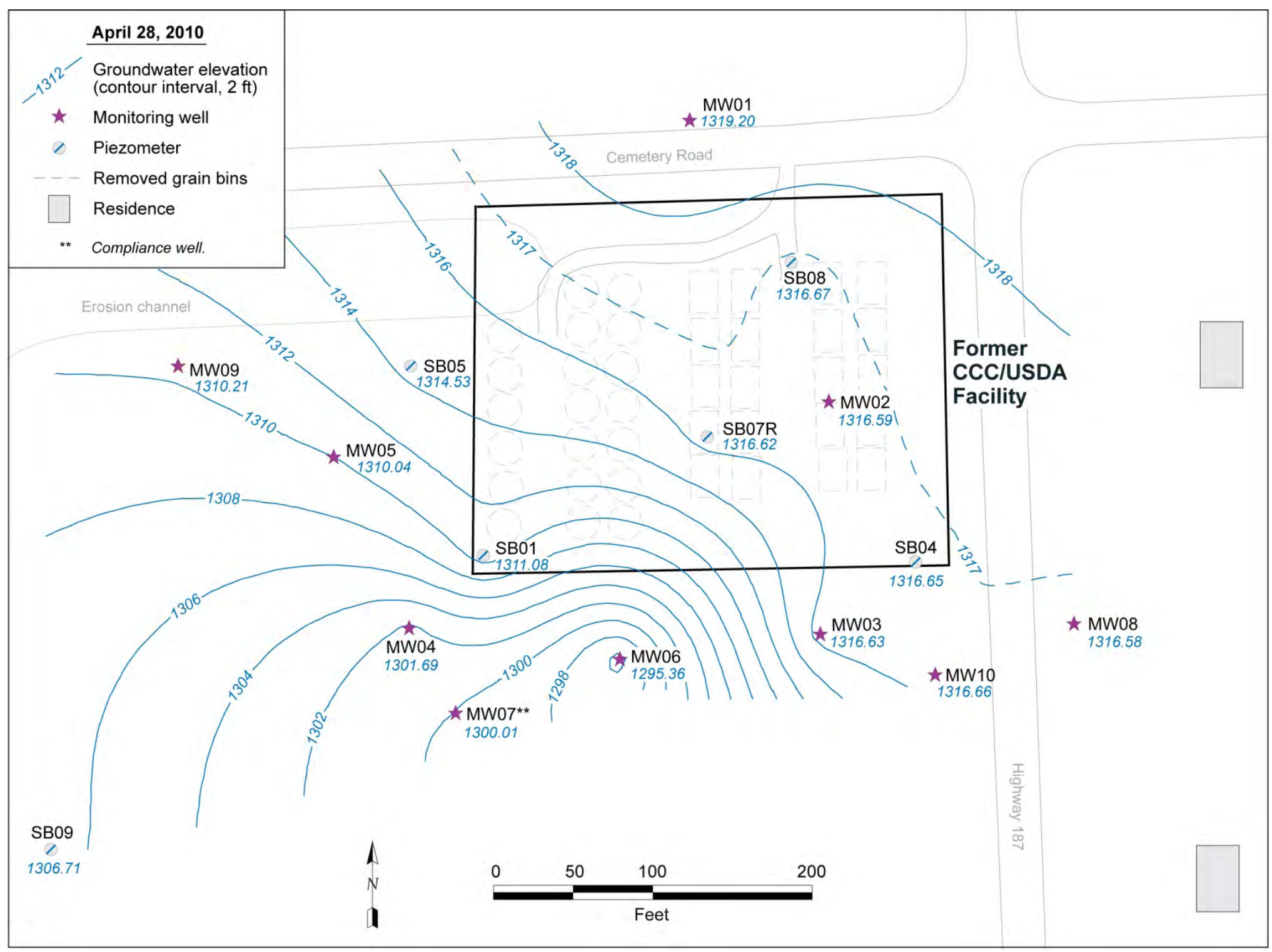

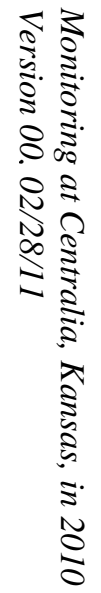

FIGURE 3.2 Potentiometric surface at Centralia, based on water levels measured manually on April 28, 2010. 


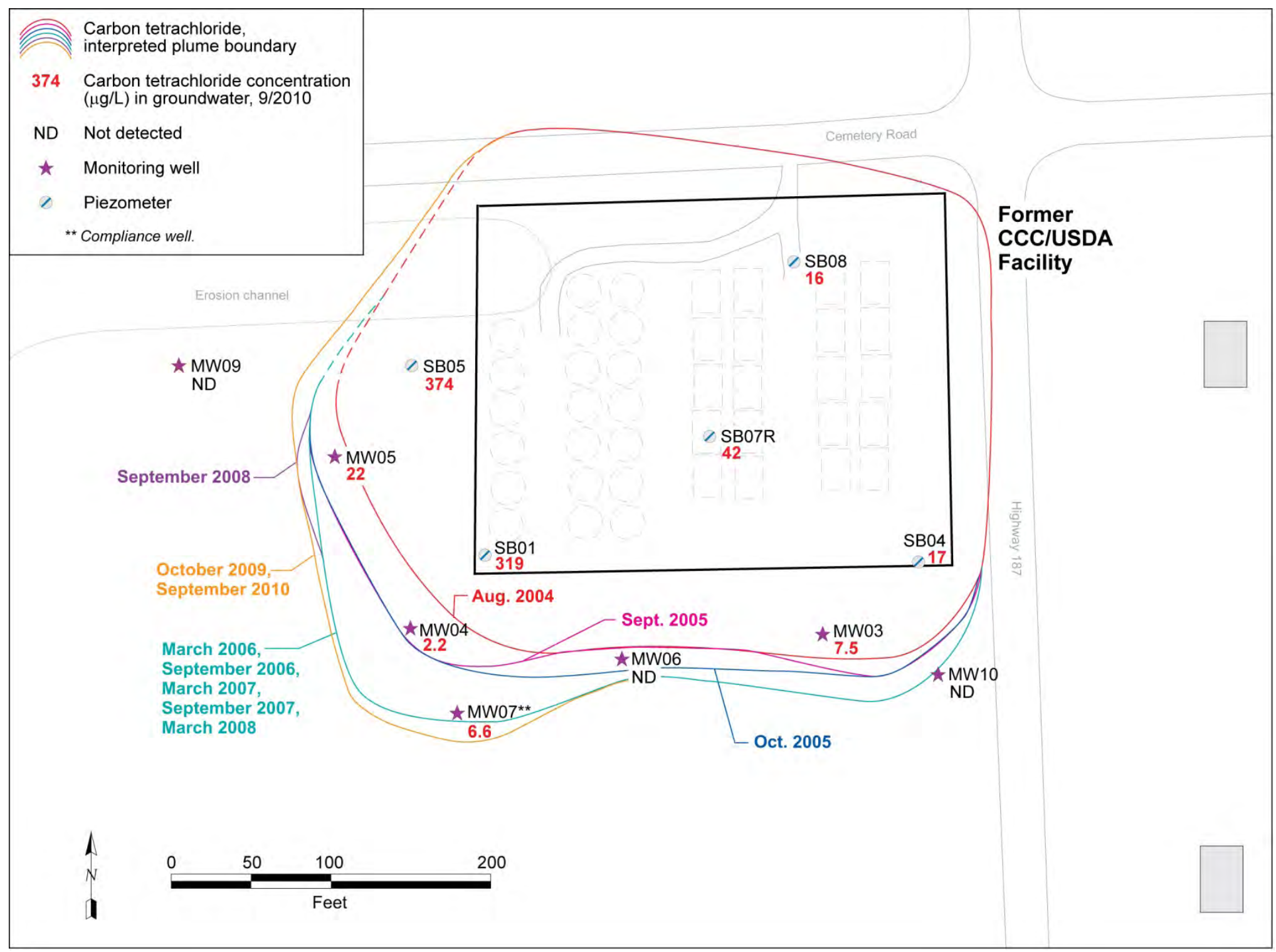

FIGURE 3.3 Carbon tetrachloride concentrations in groundwater in the sitewide monitoring wells sampled in September 2010, with the interpreted lateral extent of the contaminant at intervals during the period August 2004 to September 2010. 


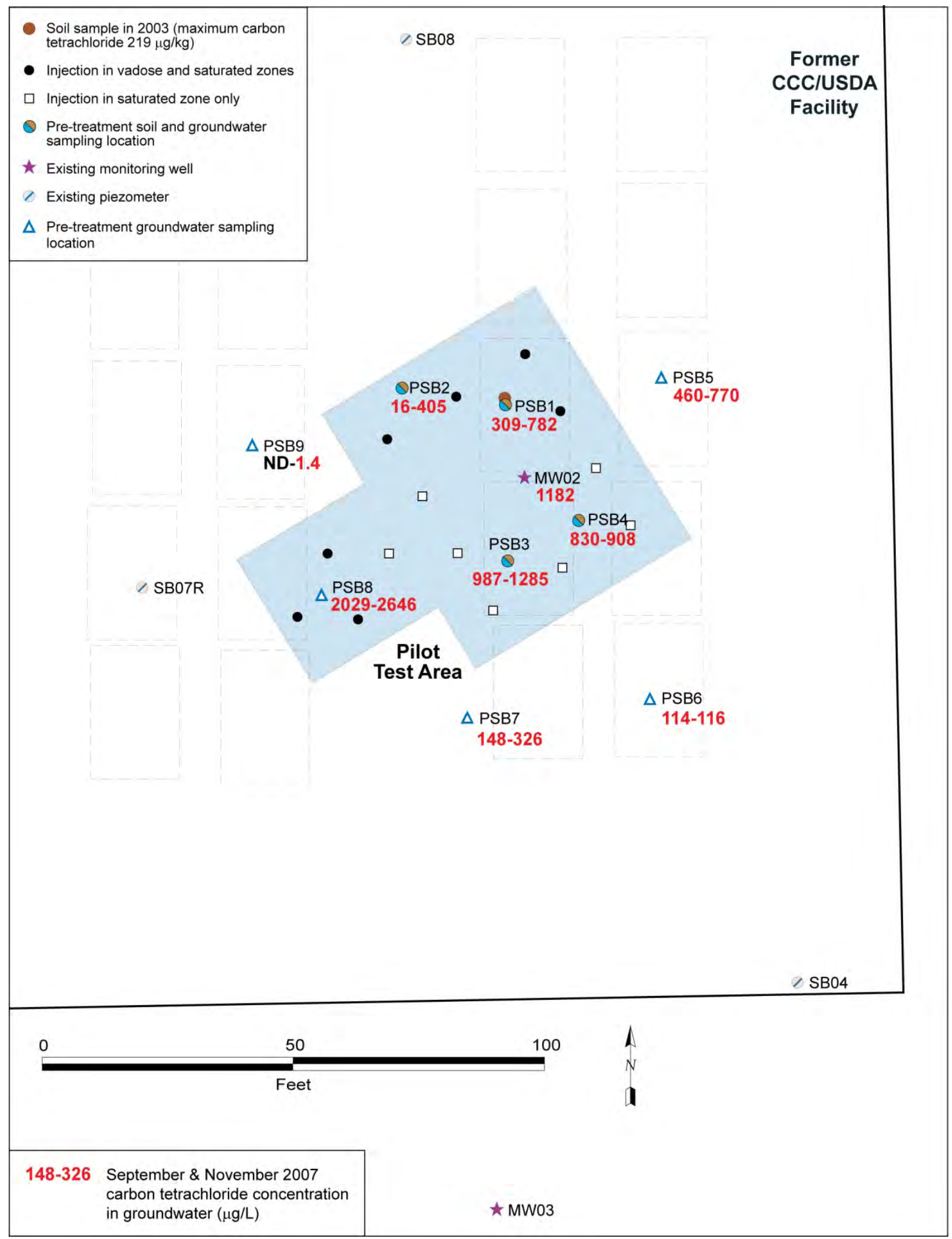

FIGURE 3.4 Carbon tetrachloride in groundwater samples collected during the pre-injection baseline sampling, September and November 2007. 


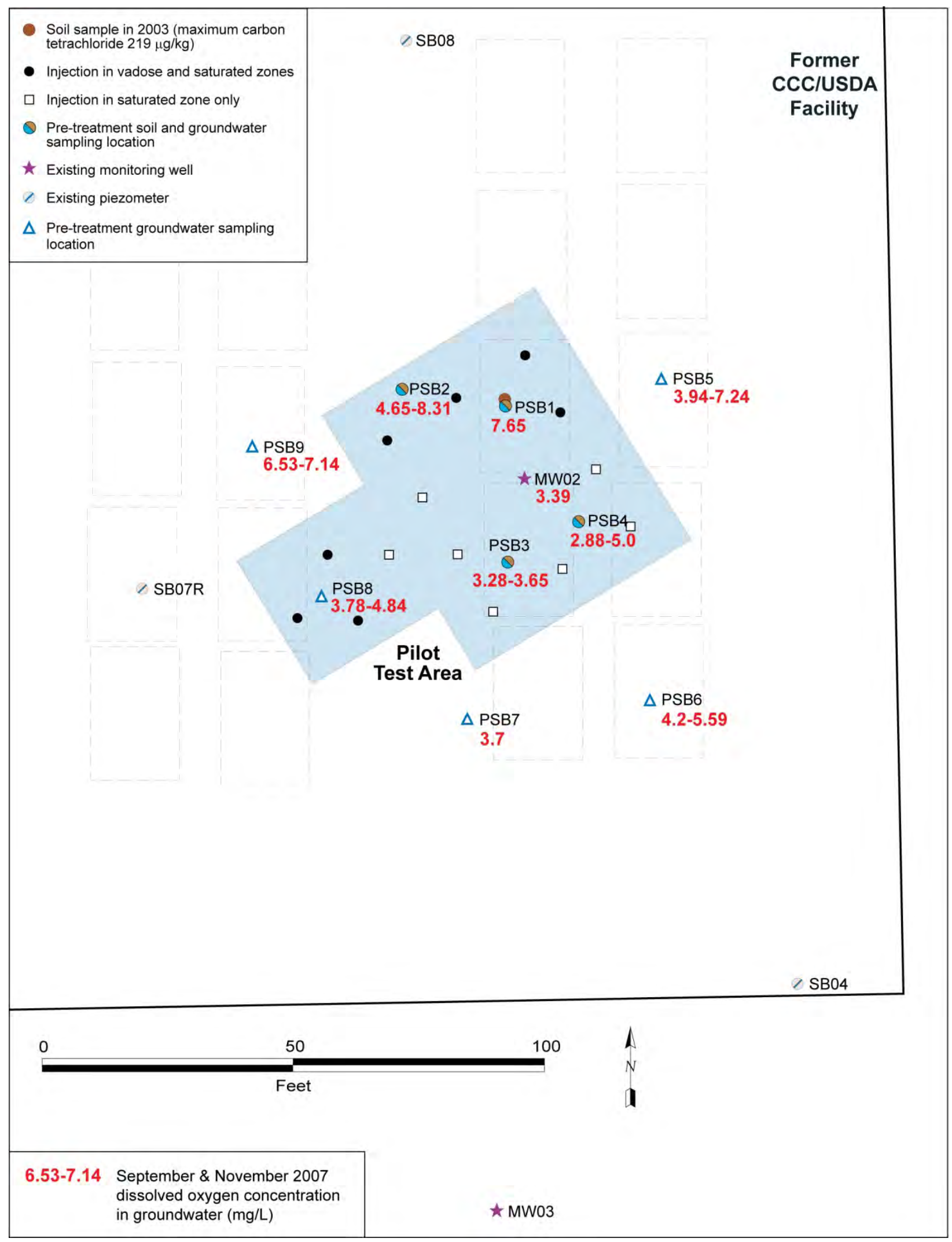

FIGURE 3.5 Field-measured results for DO in groundwater samples collected during the pre-injection baseline sampling, September and November 2007. 


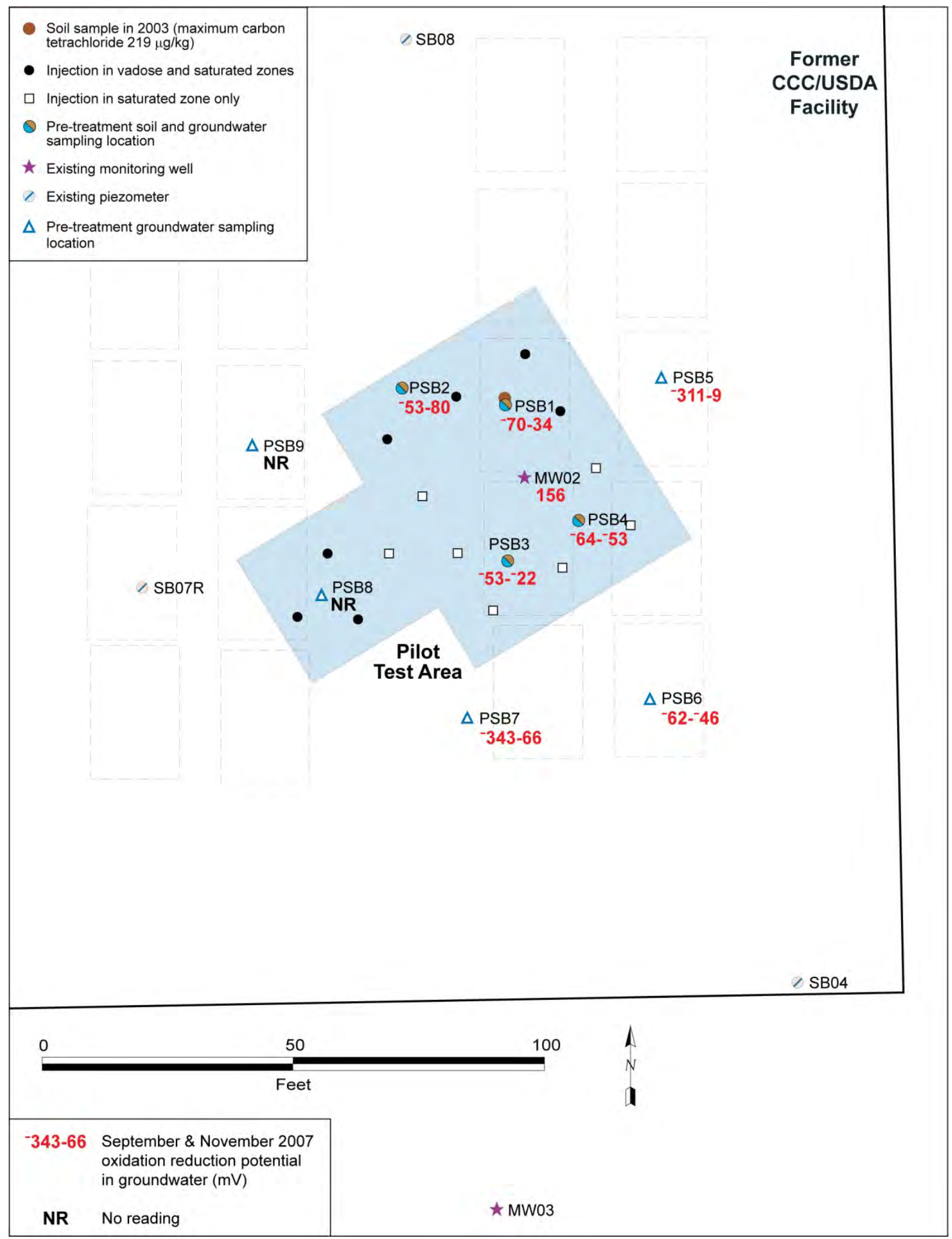

FIGURE 3.6 Field-measured results for ORP in groundwater samples collected during the pre-injection baseline sampling, September and November 2007. 


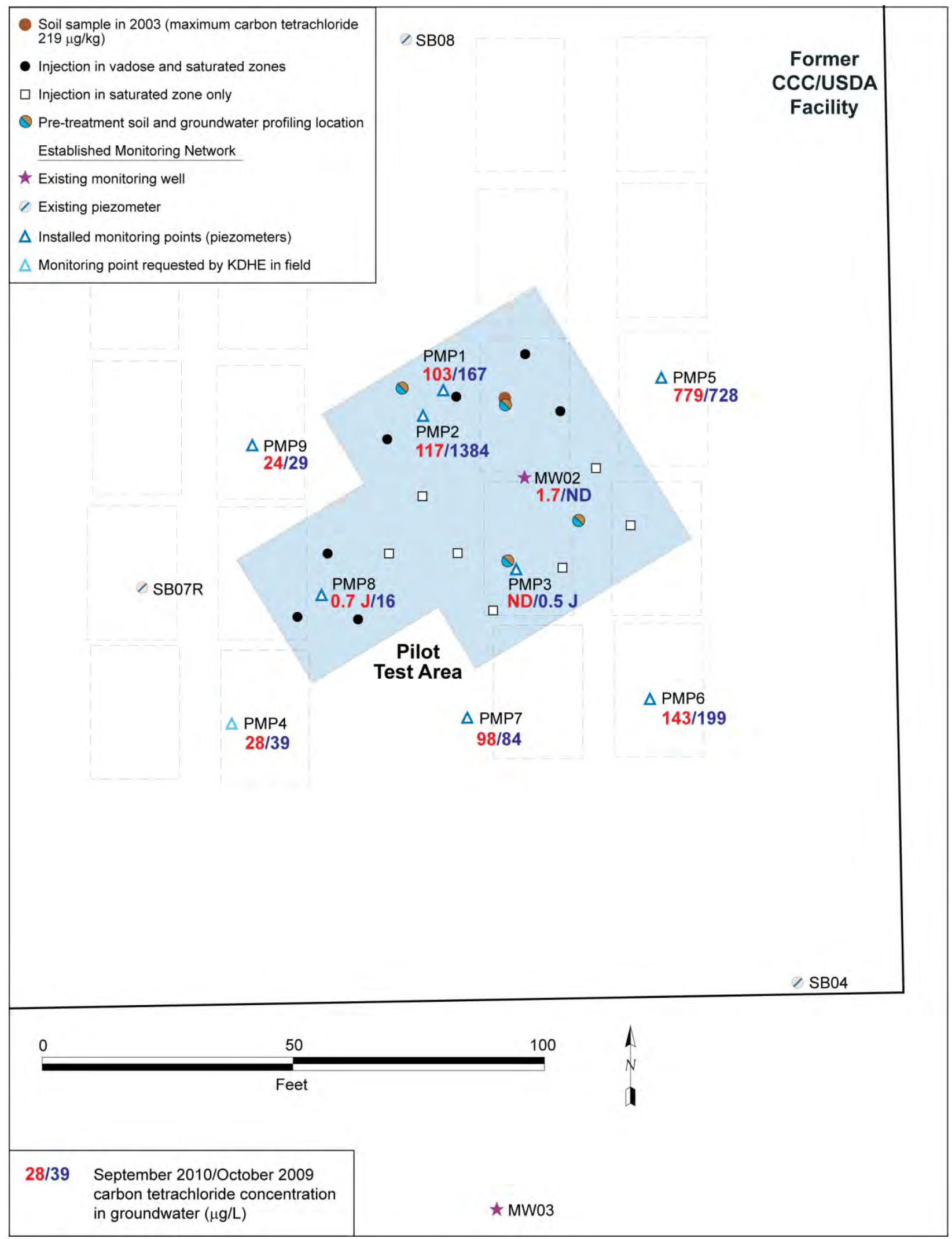

FIGURE 3.7 Analytical results for carbon tetrachloride in groundwater samples collected in September 2010 and October 2009 at the IM pilot test monitoring points. 


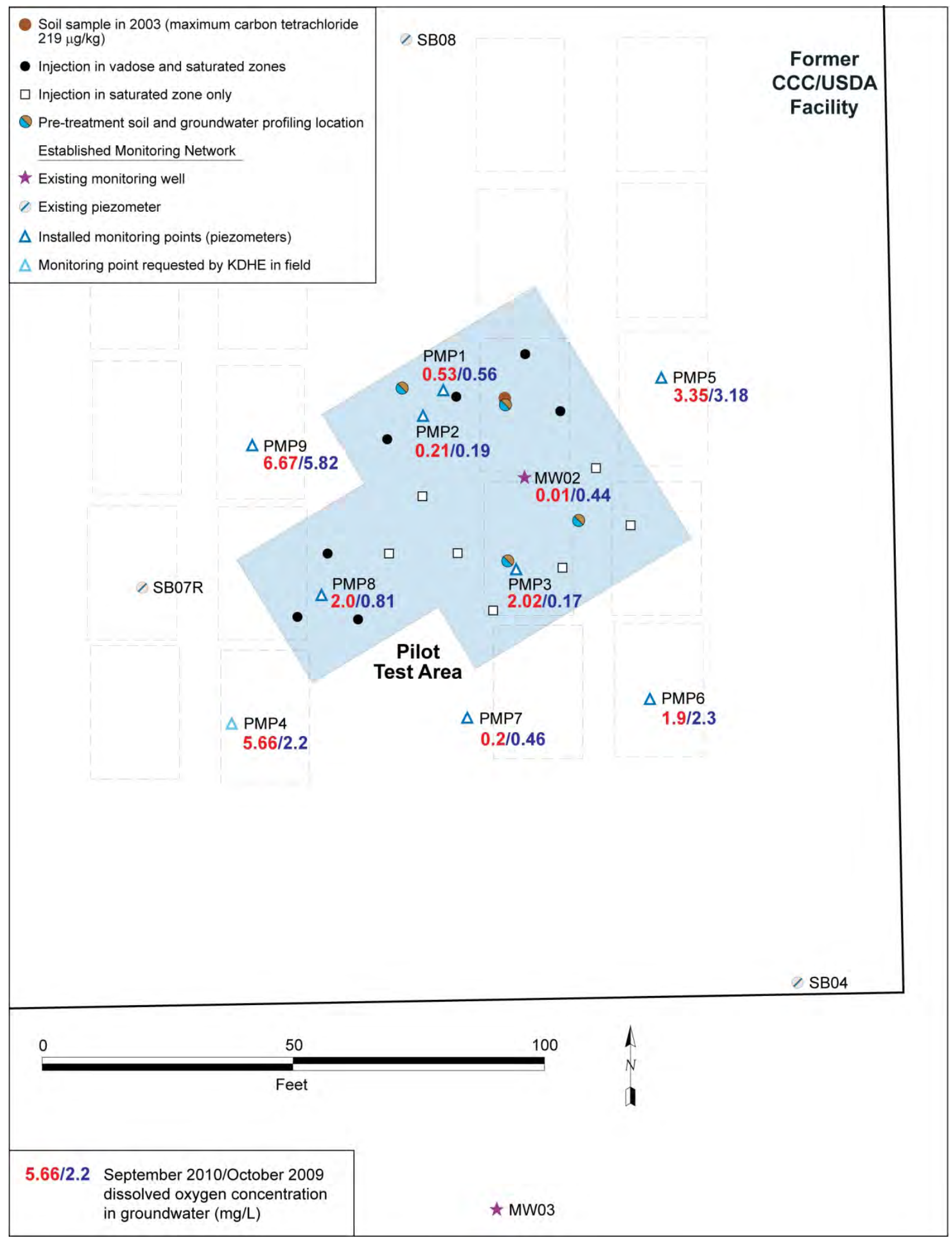

FIGURE 3.8 Field-measured results for DO in groundwater samples collected in September 2010 and October 2009 at the IM pilot test monitoring points. 


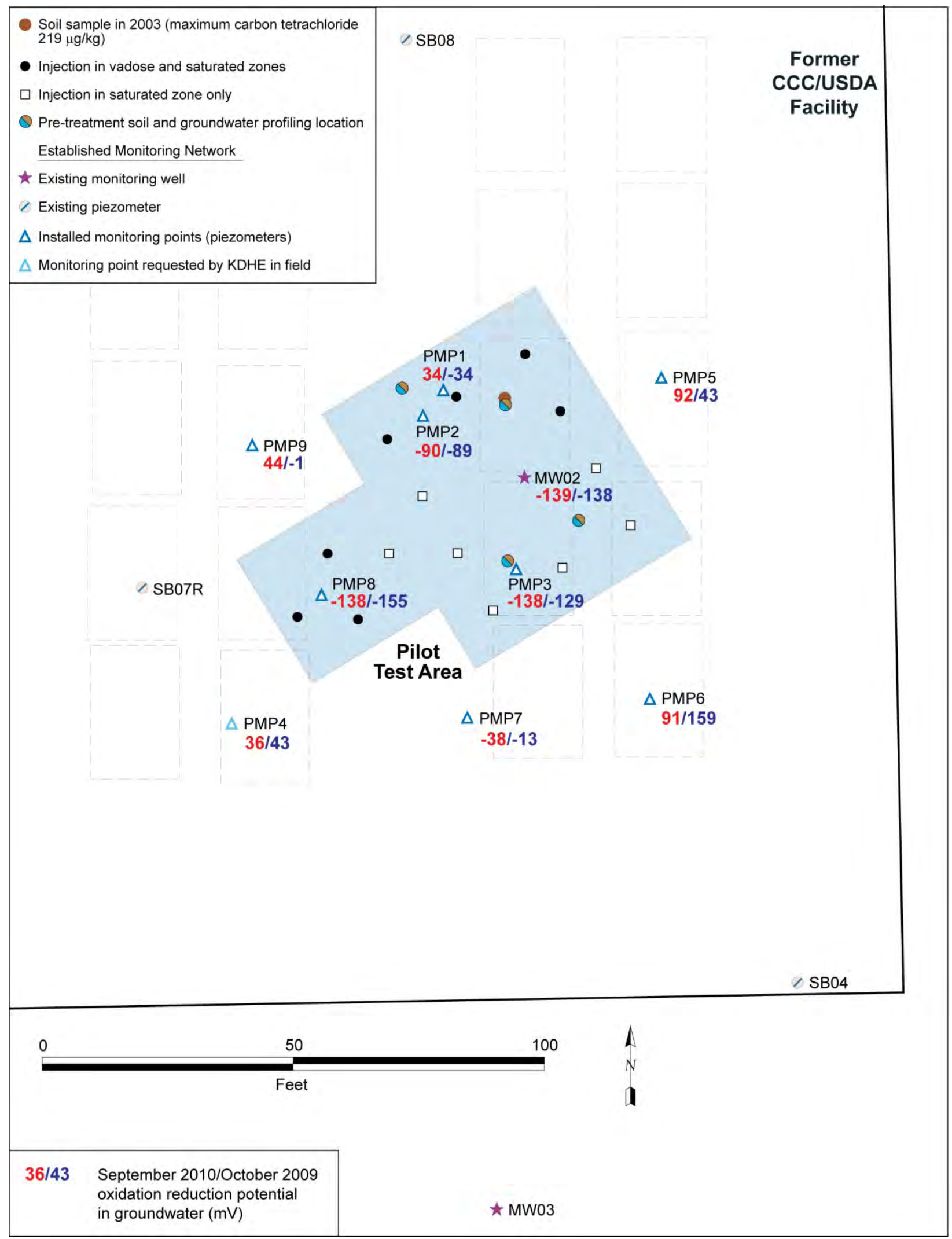

FIGURE 3.9 Field-measured results for ORP in groundwater samples collected in September 2010 and October 2009 at the IM pilot test monitoring points. 


\section{Conclusions and Recommendations}

\subsection{Conclusions}

The findings of the sitewide monitoring in September 2010 support the following conclusions for the wider investigation area:

- Manual and automated measurements of groundwater levels continued to indicate a groundwater flow direction to the south-southwest across the former CCC/USDA facility. After six years of continuous automated measurement of groundwater levels, the pressure sensors and data loggers were removed from the site after final downloads on April 5 and September 12, 2010.

- The September 2010 carbon tetrachloride data for monitoring points in the approved sitewide network were generally consistent with previous results. Continuing longer-term trends of slightly increasing carbon tetrachloride concentrations along the western and southern margins of the contaminant distribution in groundwater suggest very slow downgradient expansion of the plume.

- The presence of trace to relatively low levels of chloroform at all but one of the sitewide monitoring points having detectable levels of carbon tetrachloride suggests that some degradation of carbon tetrachloride is occurring at these locations.

- The relatively high DO concentrations and positive ORP levels identified at the sitewide monitoring points indicate that —notwithstanding the observed chloroform concentrations - anaerobic reducing conditions conducive to the reductive dechlorination of carbon tetrachloride are not widely developed sitewide.

- Although the low DO concentrations and negative ORP levels detected at monitoring well MW06 in September 2008 and October 2009 hinted at possible development of increasingly anaerobic reducing conditions at this location, such values did not persist in the current review period. The 
variability in these parameters (particularly the negative ORP levels) is somewhat greater at MW06 than at other monitoring locations, for reasons that are not clear.

The findings of the IM pilot test monitoring in April and September 2010 support the following conclusions for the pilot test area:

- The results of sampling in April and September 2010 indicate that the concentrations of carbon tetrachloride identified in groundwater in the IM pilot test injection field remained low or continued to decrease. The most significant reduction (by approximately an order of magnitude) occurred at piezometer PMP2. This is the location where a large increase in carbon tetrachloride concentrations was observed after ISCR injection (Section 4.2.5 in Argonne 2009a). The concentration at this location has now returned to approximately the pre-injection value.

- The 2010 results confirmed that relatively oxygen-depleted, chemically reducing conditions favorable to the degradation of carbon tetrachloride via reductive dechlorination persist in the injection field as a result of the ISCR injections in November 2007. The apparent lifetime (3 yr to date) suggested by these observations for the ISCR material is in the range of 1-5 yr estimated by the manufacturer (Adventus references cited in Argonne 2007b).

- Decreases in DO and ORP values observed from September 2008 to October 2009 at monitoring locations immediately southwest and downgradient of the pilot test injection field suggested that the range of influence of the injected ISCR treatment technology might be slowly increasing with time, in the direction of natural groundwater flow. Data from the 2010 sampling events did not, however, confirm this trend. 


\subsection{Recommendations}

The groundwater sampling conducted at Centralia in April and September 2010 represented the second year of monitoring performed under the interim site monitoring plan (Section 1) approved by the KDHE (2009). The results support the following recommendations:

- Analytical results continue to indicate that groundwater movement and contaminant migration are slow and predictable. These findings demonstrate that the present KDHE-approved frequency for monitoring of the groundwater at Centralia is sufficient to remain protective of human health and the environment.

- Continued monitoring in the pilot test area is appropriate, because the injected ISCR material is still active. The full effects of the treatment and the lifetime of the material under the subsurface conditions at Centralia remain to be determined.

- Continued monitoring is needed to evaluate and confirm observations made in the 3 yr of monitoring after treatment. Examples are as follows:

- More time is needed to test the hypothesis (suggested by geochemical and contaminant concentrations observed in 2009) that the range of influence of the ISCR material is expanding slowly with time in the direction of natural groundwater flow.

- The concentration at location PMP2, where contaminant levels rose significantly after injection, is still changing rapidly. Whether these concentrations reach a stable level and what that level might be are issues of interest.

- Manual water level measurements in conjunction with groundwater sampling will be adequate to confirm the established groundwater flow direction.

- In keeping with the approved interim monitoring program, sampling will occur in 2011 on the following schedule: 
- April 2011 - Sampling at IM pilot test monitoring points PMP1-PMP3, PMP8, and MW02 (Figure 1.2) inside the injection area.

- September 2011 - Sampling at sitewide monitoring points MW03MW07, MW09, MW10, SB01, SB04, SB05, SB07R, and SB08 (Figure 1.1), as well as at IM pilot test monitoring points PMP1-PMP9 and MW02 (Figure 1.2). 


\section{References}

Argonne, 2002, Final Master Work Plan: Environmental Investigations at Former CCC/USDA Facilities in Kansas, 2002 Revision, ANL/ER/TR-02/004, prepared for the Commodity Credit Corporation, U.S. Department of Agriculture, Washington, D.C., by Argonne National Laboratory, Argonne, Illinois, December.

Argonne, 2003, Final Phase I Report and Phase II Work Plan: QuickSite ${ }^{\circledR}$ Investigation, Centralia, Kansas, ANL/ER/TR-02/009, prepared for the Commodity Credit Corporation, U.S. Department of Agriculture, Washington, D.C., by Argonne National Laboratory, Argonne, Illinois, March.

Argonne, 2004, Final Phase II Report: QuickSite ${ }^{\circledR}$ Investigation, Centralia, Kansas, ANL/ER/TR-03/006, prepared for the Commodity Credit Corporation, U.S. Department of Agriculture, Washington, D.C., by Argonne National Laboratory, Argonne, Illinois, March.

Argonne, 2005a, Final Report: 2004 Monitoring Well Installation and Sampling at Centralia, Kansas, ANL/ER/TR-04/011, prepared for the Commodity Credit Corporation, U.S. Department of Agriculture, Washington, D.C., by Argonne National Laboratory, Argonne, Illinois, October.

Argonne, 2005b, Final Work Plan: Groundwater Monitoring at Centralia, Kansas, ANL/ER/TR-05/004, prepared for the Commodity Credit Corporation, U.S. Department of Agriculture, Washington, D.C., by Argonne National Laboratory, Argonne, Illinois, August.

Argonne, 2006, Final Report: Groundwater Monitoring at Centralia, Kansas, in SeptemberOctober 2005 and March 2006, with Expansion of the Monitoring Network in January 2006, ANL/EVS/AGEM/TR-06-06, prepared for the Commodity Credit Corporation, U.S. Department of Agriculture, Washington, D.C., by Argonne National Laboratory, Argonne, Illinois, October.

Argonne, 2007a, March 2007 Monitoring Results for Centralia, Kansas, ANL/EVS/AGEM/TR07-08, prepared for the Commodity Credit Corporation, U.S. Department of Agriculture, Washington, D.C., by Argonne National Laboratory, Argonne, Illinois, June.

Argonne, 2007b, Interim Measure Conceptual Design for Remediation at the Former CCC/USDA Grain Storage Facility at Centralia, Kansas: Pilot Test and Remedy 
Implementation, ANL/EVS/AGEM/TR-07-11, prepared for the Commodity Credit Corporation, U.S. Department of Agriculture, Washington, D.C., by Argonne National Laboratory, Argonne, Illinois, October.

Argonne, 2008a, September 2007 Monitoring Results for Centralia, Kansas, ANL/EVS/AGEM/TR-08-01, prepared for the Commodity Credit Corporation, U.S. Department of Agriculture, Washington, D.C., by Argonne National Laboratory, Argonne, Illinois, January.

Argonne, 2008b, March 2008 Monitoring Results for Centralia, Kansas, ANL/EVS/AGEM/TR08-08, prepared for the Commodity Credit Corporation, U.S. Department of Agriculture, Washington, D.C., by Argonne National Laboratory, Argonne, Illinois, May.

Argonne, 2009a, Progress Report and Technical Evaluation of the ISCR Pilot Test Conducted at the Former CCC/USDA Grain Storage Facility in Centralia, Kansas, ANL/EVS/AGEM/TR-0818, prepared for the Commodity Credit Corporation, U.S. Department of Agriculture, Washington, D.C., by Argonne National Laboratory, Argonne, Illinois, January.

Argonne, 2009b, September 2008 Monitoring Results for Centralia, Kansas, ANL/EVS/AGEM/TR-09-01, prepared for the Commodity Credit Corporation, U.S. Department of Agriculture, Washington, D.C., by Argonne National Laboratory, Argonne, Illinois, February.

Argonne, 2010, Annual Report of Groundwater Monitoring at Centralia, Kansas, in 2009, ANL/EVS/AGEM/TR-10-07, prepared for the Commodity Credit Corporation, U.S. Department of Agriculture, Washington, D.C., by Argonne National Laboratory, Argonne, Illinois, October.

EPA, 1995, Method 524.2: Measurement of Purgeable Organic Compounds in Water by Capillary Column Gas Chromatography/Mass Spectrometry, Revision 4.1, edited by J.W. Munch, National Exposure Research Laboratory, Office of Research and Development, U.S. Environmental Protection Agency, Cincinnati, Ohio.

KDHE, 2007, letter from C. Carey (Bureau of Environmental Remediation, Kansas Department of Health and Environment, Topeka, Kansas) to C. Roe (Commodity Credit Corporation, U.S. Department of Agriculture, Washington, D.C.), regarding review of Interim Measure Conceptual Design for Centralia, Kansas, November 9. 
KDHE, 2008a, letter from C. Carey (Bureau of Environmental Remediation, Kansas Department of Health and Environment, Topeka, Kansas) to C. Roe (Commodity Credit Corporation, U.S. Department of Agriculture, Washington, D.C.), regarding September 2007 Monitoring Results for Centralia, Kansas, March 12.

KDHE, 2008b, electronic mail message from C. Carey (Bureau of Environmental Remediation, Kansas Department of Health and Environment, Topeka, Kansas) to L. LaFreniere (Argonne National Laboratory, Argonne, Illinois), regarding use of the low-flow sampling technique at Centralia, February 11.

KDHE, 2009, letter from E. Finzer (Bureau of Environmental Remediation, Kansas Department of Health and Environment, Topeka, Kansas) to C. Roe (Commodity Credit Corporation, U.S. Department of Agriculture, Washington, D.C.), regarding Centralia monitoring reports for 2008 and the progress report for the ISCR pilot test, April 6.

Puls, R.W., and M.J. Barcelona, 1996, "Low-Flow (Minimal Drawdown) Ground-Water Sampling Procedures,” EPA/540/S-95/504, in Ground Water Issue, Superfund Technology Support Center for Ground Water, National Risk Management Research Laboratory, Ada, Oklahoma, April (www.epa.gov/tio/tsp/download/lwflw2a.pdf). 


\section{Appendix A:}

Sequence of Sampling Activities in 2010 
TABLE A.1 Sequence of sampling activities at Centralia in 2010.

\begin{tabular}{|c|c|c|c|c|c|c|c|c|}
\hline $\begin{array}{l}\text { Sample } \\
\text { Date }\end{array}$ & Time & Sample & $\begin{array}{l}\text { Sample } \\
\text { Type }^{a}\end{array}$ & Location & $\begin{array}{l}\text { Depth } \\
\text { (ft BGL) }\end{array}$ & $\begin{array}{l}\text { Chain of } \\
\text { Custody }\end{array}$ & $\begin{array}{l}\text { Shipping } \\
\text { Date }\end{array}$ & Sample Description \\
\hline \multicolumn{9}{|c|}{ April 2010 monitoring } \\
\hline $4 / 5 / 10$ & $18: 32$ & CNPMP1-W-27180 & MW & PMP1 & $50-60$ & 2623 & $4 / 6 / 10$ & $\begin{array}{l}\text { Depth to water }=17.25 \mathrm{ft} \text {. Depth of } 0.5 \text {-in. } \\
\text { well }=60 \mathrm{ft} \text {. Sample collected by using a } \\
\text { Waterra pump positioned at } 55 \mathrm{ft} \text { after } \\
\text { purging of } 5.2 \mathrm{~L} \text {. }\end{array}$ \\
\hline $4 / 5 / 10$ & $18: 42$ & CNMW02-W-27179 & MW & MW02 & $49.5-59.5$ & 2623 & $4 / 6 / 10$ & $\begin{array}{l}\text { Depth to water = } 18.7 \mathrm{ft} \text {. Depth of } 4 \text {-in. well } \\
=59.5 \mathrm{ft} \text {. Sample collected by using a } \\
\text { low-flow bladder pump positioned at } \\
54.5 \mathrm{ft} \text { after purging of } 8 \mathrm{~L} \text {. }\end{array}$ \\
\hline $4 / 5 / 10$ & $18: 50$ & CNQCIR-W-27184 & RI & QC & - & 2623 & $4 / 6 / 10$ & $\begin{array}{l}\text { Rinsate of decontaminated sampling line } \\
\text { after collection of sample CNMWO2-W- } \\
27179 \text {. }\end{array}$ \\
\hline $4 / 5 / 10$ & $19: 10$ & CNPMP2-W-27181 & MW & PMP2 & $50-60$ & 2623 & $4 / 6 / 10$ & $\begin{array}{l}\text { Depth to water }=17.16 \mathrm{ft} \text {. Depth of } 0.5 \text {-in. } \\
\text { well }=60 \mathrm{ft} \text {. Sample collected by using a } \\
\text { Waterra pump positioned at } 55 \mathrm{ft} \text { after } \\
\text { purging of } 5.3 \mathrm{~L} \text {. }\end{array}$ \\
\hline $4 / 5 / 10$ & $19: 40$ & CNPMP3-W-27182 & MW & PMP3 & $50-60$ & 2623 & $4 / 6 / 10$ & $\begin{array}{l}\text { Depth to water }=17.9 \mathrm{ft} \text {. Depth of } 0.5 \text {-in. } \\
\text { well }=60 \mathrm{ft} \text {. Sample collected by using a } \\
\text { Waterra pump positioned at } 55 \mathrm{ft} \text { after } \\
\text { purging of } 5.75 \mathrm{~L} \text {. }\end{array}$ \\
\hline $4 / 5 / 10$ & $20: 05$ & CNPMP8-W-27183 & MW & PMP8 & $50-60$ & 2623 & $4 / 6 / 10$ & $\begin{array}{l}\text { Depth to water }=16.12 \mathrm{ft} \text {. Depth of } 0.5 \text {-in. } \\
\text { well }=60 \mathrm{ft} \text {. Sample collected by using a } \\
\text { Waterra pump positioned at } 55 \mathrm{ft} \text { after } \\
\text { purging of } 5.3 \mathrm{~L} \text {. }\end{array}$ \\
\hline $4 / 5 / 10$ & $20: 30$ & CNQCTB-W-27185 ${ }^{\mathrm{b}}$ & TB & $\mathrm{QC}$ & - & 2623 & $4 / 6 / 10$ & $\begin{array}{l}\text { Trip blank sent to the AGEM Laboratory for } \\
\text { organic analysis with water samples } \\
\text { listed on chain-of-custody form (COC) } \\
2623 \text {. }\end{array}$ \\
\hline \multicolumn{9}{|c|}{ September 2010 monitoring } \\
\hline 9/19/10 & $15: 06$ & CNMW08-W-27193 & MW & MW08 & $38-53$ & 2689 & 9/20/10 & $\begin{array}{l}\text { Depth to water }=17.52 \mathrm{ft} \text {. Depth of } 2 \text {-in. } \\
\text { well }=53 \mathrm{ft} \text {. Sample collected by using a } \\
\text { low-flow bladder pump positioned at } \\
45.5 \mathrm{ft} \text { after purging of } 7.5 \mathrm{~L} \text {. }\end{array}$ \\
\hline
\end{tabular}


TABLE A.1 (Cont.)

\begin{tabular}{|c|c|c|c|c|c|c|c|c|}
\hline $\begin{array}{c}\text { Sample } \\
\text { Date }\end{array}$ & Time & Sample & $\begin{array}{c}\text { Sample } \\
\text { Type }^{a}\end{array}$ & Location & $\begin{array}{l}\text { Depth } \\
\text { (ft BGL) }\end{array}$ & $\begin{array}{l}\text { Chain of } \\
\text { Custody }\end{array}$ & $\begin{array}{c}\text { Shipping } \\
\text { Date }\end{array}$ & Sample Description \\
\hline \multicolumn{9}{|c|}{ September 2010 monitoring (cont.) } \\
\hline 9/19/10 & $15: 42$ & CNSB09-W-27201 & $\mathrm{CPT/P}$ & SB09 & $32-42$ & 2689 & $9 / 20 / 10$ & $\begin{array}{l}\text { Depth to water }=6.78 \mathrm{ft} \text {. Depth of } 1 \text {-in. well } \\
=42 \mathrm{ft} \text {. Sample collected by using a } \\
\text { low-flow bladder pump positioned at } 37 \\
\mathrm{ft} \text { after purging of } 2 \mathrm{~L} \text {. }\end{array}$ \\
\hline $9 / 19 / 10$ & $16: 10$ & CNMW10-W-27195 & MW & MW10 & $30-45$ & 2689 & $9 / 20 / 10$ & $\begin{array}{l}\text { Depth to water }=19.92 \mathrm{ft} \text {. Depth of } 2 \text {-in. } \\
\text { well }=45 \mathrm{ft} \text {. Sample collected by using a } \\
\text { low-flow bladder pump positioned at } \\
37.5 \mathrm{ft} \text { after purging of } 9 \mathrm{~L} \text {. }\end{array}$ \\
\hline $9 / 19 / 10$ & $16: 51$ & CNMW09-W-27194 & MW & MW09 & $25-35$ & 2689 & $9 / 20 / 10$ & $\begin{array}{l}\text { Depth to water }=2.66 \mathrm{ft} \text {. Depth of } 2 \text {-in. well } \\
=35 \mathrm{ft} \text {. Sample collected by using a } \\
\text { low-flow bladder pump positioned at } \\
30 \mathrm{ft} \text { after purging of } 7 \mathrm{~L} \text {. }\end{array}$ \\
\hline $9 / 19 / 10$ & $16: 52$ & CNMW09DUP-W-27212 & MW & MW09 & 25-35 & 2689 & $9 / 20 / 10$ & Replicate of sample CNMW09-W-27194. \\
\hline 9/19/10 & $17: 02$ & CNMW03-W-27188 & MW & MW03 & $50.5-60.5$ & 2689 & $9 / 20 / 10$ & $\begin{array}{l}\text { Depth to water = } 19.42 \mathrm{ft} \text {. Depth of } 4 \text {-in. } \\
\text { well }=60.5 \mathrm{ft} \text {. Sample collected by using } \\
\text { a low-flow bladder pump positioned at } \\
55.5 \mathrm{ft} \text { after purging of } 8 \mathrm{~L} \text {. }\end{array}$ \\
\hline $9 / 19 / 10$ & $17: 03$ & CNMW03DUP-W-27211 ${ }^{\mathrm{b}}$ & MW & MW03 & $50.5-60.5$ & 2689 & $9 / 20 / 10$ & Replicate of sample CNMW03-W-27188. \\
\hline $9 / 20 / 10$ & $11: 06$ & CNMW07-W-27192 & MW & MW07 & $45-55$ & 2689 & 9/20/10 & $\begin{array}{l}\text { Depth to water }=25.03 \mathrm{ft} \text {. Depth of } 2 \text {-in. } \\
\text { well }=55 \mathrm{ft} \text {. Sample collected by using a } \\
\text { low-flow bladder pump positioned at } \\
50 \mathrm{ft} \text { after purging of } 4 \mathrm{~L} \text {. }\end{array}$ \\
\hline $9 / 20 / 10$ & $11: 44$ & CNMW06-W-27191 & MW & MW06 & $46.5-56.5$ & 2689 & 9/20/10 & $\begin{array}{l}\text { Depth to water }=34.96 \text {. ft. Depth of } 4 \text {-in. } \\
\text { well }=56.5 \mathrm{ft} \text {. Sample collected by using } \\
\text { a low-flow bladder pump positioned at } \\
51.5 \mathrm{ft} \text { after purging of } 20 \mathrm{~L} \text {. }\end{array}$ \\
\hline $9 / 20 / 10$ & $12: 21$ & CNMW04-W-27189 & MW & MW04 & $37.5-47.5$ & 2689 & $9 / 20 / 10$ & $\begin{array}{l}\text { Depth to water }=22.42 \mathrm{ft} \text {. Depth of } 4 \text {-in. } \\
\text { well }=47.5 \mathrm{ft} \text {. Sample collected by using } \\
\text { a low-flow bladder pump positioned at } \\
42.5 \mathrm{ft} \text { after purging of } 13 \mathrm{~L} \text {. }\end{array}$ \\
\hline $9 / 20 / 10$ & $12: 42$ & CNMW02-W-27187 & MW & MW02 & $49.5-59.5$ & 2689 & $9 / 20 / 10$ & $\begin{array}{l}\text { Depth to water }=19.72 \mathrm{ft} \text {. Depth of } 4 \text {-in. } \\
\text { well }=59.5 \mathrm{ft} \text {. Sample collected by using } \\
\text { a low-flow bladder pump positioned at } \\
54.5 \mathrm{ft} \text { after purging of } 9 \mathrm{~L} \text {. }\end{array}$ \\
\hline
\end{tabular}


TABLE A.1 (Cont.)

\begin{tabular}{|c|c|c|c|c|c|c|c|c|}
\hline $\begin{array}{l}\text { Sample } \\
\text { Date }\end{array}$ & Time & Sample & $\begin{array}{c}\text { Sample } \\
\text { Type }^{a}\end{array}$ & Location & $\begin{array}{l}\text { Depth } \\
\text { (ft BGL) }\end{array}$ & $\begin{array}{l}\text { Chain of } \\
\text { Custody }\end{array}$ & $\begin{array}{l}\text { Shipping } \\
\text { Date }\end{array}$ & Sample Description \\
\hline \multicolumn{9}{|c|}{ September 2010 monitoring (cont.) } \\
\hline $9 / 20 / 10$ & $12: 42$ & CNQCTB-W-27216 b & TB & QC & - & 2689 & $9 / 20 / 10$ & $\begin{array}{l}\text { Trip blank sent to the AGEM Laboratory for } \\
\text { organic analysis with water samples } \\
\text { listed on COC } 2689 \text {. }\end{array}$ \\
\hline $9 / 20 / 10$ & $13: 24$ & CNMW05-W-27190 & MW & MW05 & $34.5-44.5$ & 2717 & $9 / 21 / 10$ & $\begin{array}{l}\text { Depth to water }=10.38 \mathrm{ft} \text {. Depth of } 4 \text {-in. } \\
\text { well }=44.5 \mathrm{ft} \text {. Sample collected by using } \\
\text { a low-flow bladder pump positioned at } \\
39.5 \mathrm{ft} \text { after purging of } 8 \mathrm{~L} \text {. }\end{array}$ \\
\hline $9 / 20 / 10$ & $13: 40$ & CNMW01-W-27186 & MW & MW01 & $54.5-64.5$ & 2717 & $9 / 21 / 10$ & $\begin{array}{l}\text { Depth to water }=12.17 \mathrm{ft} \text {. Depth of } 4 \text {-in. } \\
\text { well }=64.5 \mathrm{ft} \text {. Sample collected by using } \\
\text { a low-flow bladder pump positioned at } \\
59.5 \mathrm{ft} \text { after purging of } 9.5 \mathrm{~L} \text {. }\end{array}$ \\
\hline $9 / 20 / 10$ & $13: 50$ & CNQCIR-W-27214 & RI & QC & - & 2717 & $9 / 21 / 10$ & $\begin{array}{l}\text { Rinsate of decontaminated sampling line } \\
\text { after collection of sample CNMW05-W- } \\
27190 \text {. }\end{array}$ \\
\hline $9 / 20 / 10$ & $14: 52$ & CNPMP5-W-27206 & MW & PMP5 & $50-60$ & 2717 & $9 / 21 / 10$ & $\begin{array}{l}\text { Depth to water }=20.2 \mathrm{ft} \text {. Depth of } 1 \text {-in. well } \\
=60 \mathrm{ft} \text {. Sample collected by using a } \\
\text { low-flow bladder pump positioned at } \\
55 \mathrm{ft} \text { after purging of } 3 \mathrm{~L} \text {. }\end{array}$ \\
\hline $9 / 20 / 10$ & $14: 55$ & CNSB01-W-27196 & СРТ/P & SB01 & $40-50$ & 2717 & $9 / 21 / 10$ & $\begin{array}{l}\text { Depth to water }=14.02 \mathrm{ft} \text {. Depth of } 1 \text {-in. } \\
\text { well = } 50 \mathrm{ft} \text {. Sample collected by using a } \\
\text { low-flow bladder pump positioned at } \\
45 \mathrm{ft} \text { after purging of } 2 \mathrm{~L} \text {. }\end{array}$ \\
\hline 9/20/10 & $15: 46$ & CNSB04-W-27197 & СРТ/P & SB04 & $51-61$ & 2717 & $9 / 21 / 10$ & $\begin{array}{l}\text { Depth to water }=20.42 \mathrm{ft} \text {. Depth of } 1 \text {-in. } \\
\text { well = } 61 \mathrm{ft} \text {. Sample collected by using a } \\
\text { low-flow bladder pump positioned at } \\
56 \mathrm{ft} \text { after purging of } 4.1 \mathrm{~L} \text {. }\end{array}$ \\
\hline $9 / 20 / 10$ & $16: 16$ & CNQCIR-W-27213b & RI & QC & - & 2689 & $9 / 20 / 10$ & $\begin{array}{l}\text { Rinsate of decontaminated sampling line } \\
\text { after collection of sample CNMW10-W- } \\
27195 .\end{array}$ \\
\hline $9 / 20 / 10$ & $16: 40$ & CNSB07R-W-27199 & СРТ/P & SB07R & $45-60$ & 2717 & $9 / 21 / 10$ & $\begin{array}{l}\text { Depth to water }=17.19 \mathrm{ft} \text {. Depth of } 2 \text {-in. } \\
\text { well }=60 \mathrm{ft} \text {. Sample collected by using a } \\
\text { low-flow bladder pump positioned at } \\
52.5 \mathrm{ft} \text { after purging of } 6 \mathrm{~L} \text {. }\end{array}$ \\
\hline
\end{tabular}
$52.5 \mathrm{ft}$ after purging of $6 \mathrm{~L}$. 
TABLE A.1 (Cont.)

\begin{tabular}{|c|c|c|c|c|c|c|c|c|}
\hline $\begin{array}{l}\text { Sample } \\
\text { Date }\end{array}$ & Time & Sample & $\begin{array}{c}\text { Sample } \\
\text { Type }^{a}\end{array}$ & Location & $\begin{array}{l}\text { Depth } \\
\text { (ft BGL) }\end{array}$ & $\begin{array}{l}\text { Chain of } \\
\text { Custody }\end{array}$ & $\begin{array}{l}\text { Shipping } \\
\text { Date }\end{array}$ & Sample Description \\
\hline \multicolumn{9}{|c|}{ September 2010 monitoring (cont.) } \\
\hline $9 / 20 / 10$ & $17: 30$ & CNSB08-W-27200 & СРТ/P & SB08 & $52-62$ & 2717 & $9 / 21 / 10$ & $\begin{array}{l}\text { Depth to water }=17.23 \mathrm{ft} \text {. Depth of } 1 \text {-in. } \\
\text { well }=62 \mathrm{ft} \text {. Sample collected by using a } \\
\text { low-flow bladder pump positioned at } \\
57 \mathrm{ft} \text { after purging of } 3 \mathrm{~L} \text {. }\end{array}$ \\
\hline $9 / 21 / 10$ & $10: 44$ & CNPMP1-W-27202 & MW & PMP1 & $50-60$ & 2716 & $9 / 21 / 10$ & $\begin{array}{l}\text { Depth to water }=18.65 \mathrm{ft} \text {. Depth of } 0.5 \text {-in. } \\
\text { well }=60 \mathrm{ft} \text {. Sample collected by using a } \\
\text { low-flow bladder pump positioned at } \\
55 \mathrm{ft} \text { after purging of } 6 \mathrm{~L} \text {. }\end{array}$ \\
\hline $9 / 21 / 10$ & $11: 09$ & CNPMP2-W-27203 & MW & PMP2 & $50-60$ & 2716 & $9 / 21 / 10$ & $\begin{array}{l}\text { Depth to water }=18.68 \mathrm{ft} \text {. Depth of } 0.5 \text {-in. } \\
\text { well }=60 \mathrm{ft} \text {. Sample collected by using a } \\
\text { low-flow bladder pump positioned at } \\
55 \mathrm{ft} \text { after purging of } 6 \mathrm{~L} \text {. }\end{array}$ \\
\hline $9 / 21 / 10$ & $11: 42$ & CNPMP3-W-27204 & MW & PMP3 & $50-60$ & 2716 & $9 / 21 / 10$ & $\begin{array}{l}\text { Depth to water }=19.35 \mathrm{ft} \text {. Depth of } 0.5 \text {-in. } \\
\text { well = } 60 \mathrm{ft} \text {. Sample collected by using a } \\
\text { low-flow bladder pump positioned at } \\
55 \mathrm{ft} \text { after purging of } 6 \mathrm{~L} \text {. }\end{array}$ \\
\hline $9 / 21 / 10$ & $12: 11$ & CNPMP4-W-27205 & MW & PMP4 & $48.75-58.75$ & 2716 & $9 / 21 / 10$ & $\begin{array}{l}\text { Depth to water }=16.83 \mathrm{ft} \text {. Depth of } 0.5 \text {-in. } \\
\text { well = } 58.75 \mathrm{ft} \text {. Sample collected by } \\
\text { using a low-flow bladder pump } \\
\text { positioned at } 53.75 \mathrm{ft} \text { after purging of } \\
6.5 \mathrm{~L} \text {. }\end{array}$ \\
\hline $9 / 21 / 10$ & $12: 32$ & CNPMP7-W-27208 & MW & PMP7 & $50-60$ & 2716 & $9 / 21 / 10$ & $\begin{array}{l}\text { Depth to water }=18.84 \mathrm{ft} \text {. Depth of } 0.5 \text {-in. } \\
\text { well }=60 \mathrm{ft} \text {. Sample collected by using a } \\
\text { low-flow bladder pump positioned at } \\
55 \mathrm{ft} \text { after purging of } 7 \mathrm{~L} \text {. }\end{array}$ \\
\hline $9 / 21 / 10$ & $12: 47$ & CNPMP8-W-27209 & MW & PMP8 & $50-60$ & 2716 & $9 / 21 / 10$ & $\begin{array}{l}\text { Depth to water }=17.91 \mathrm{ft} \text {. Depth of } 0.5 \text {-in. } \\
\text { well = } 60 \mathrm{ft} \text {. Sample collected by using a } \\
\text { low-flow bladder pump positioned at } \\
55 \mathrm{ft} \text { after purging of } 7 \mathrm{~L} \text {. }\end{array}$ \\
\hline $9 / 21 / 10$ & 13:11 & CNPMP9-W-27210 & MW & PMP9 & $50-60$ & 2716 & $9 / 21 / 10$ & $\begin{array}{l}\text { Depth to water }=15.30 \mathrm{ft} \text {. Depth of } 0.5 \text {-in. } \\
\text { well }=60 \mathrm{ft} \text {. Sample collected by using a } \\
\text { low-flow bladder pump positioned at } \\
55 \mathrm{ft} \text { after purging of } 6.7 \mathrm{~L} \text {. }\end{array}$ \\
\hline
\end{tabular}
$55 \mathrm{ft}$ after purging of $6.7 \mathrm{~L}$. 
TABLE A.1 (Cont.)

\begin{tabular}{|c|c|c|c|c|c|c|c|c|}
\hline $\begin{array}{l}\text { Sample } \\
\text { Date }\end{array}$ & Time & Sample & $\begin{array}{l}\text { Sample } \\
\text { Type }^{a}\end{array}$ & Location & $\begin{array}{l}\text { Depth } \\
\text { (ft BGL) }\end{array}$ & $\begin{array}{l}\text { Chain of } \\
\text { Custody }\end{array}$ & $\begin{array}{l}\text { Shipping } \\
\text { Date }\end{array}$ & Sample Description \\
\hline \multicolumn{9}{|c|}{ September 2010 monitoring (cont.) } \\
\hline $9 / 21 / 10$ & $13: 42$ & CNSB05-W-27198 & $\mathrm{CPT/P}$ & SB05 & $32-42$ & 2716 & $9 / 21 / 10$ & $\begin{array}{l}\text { Depth to water }=10.20 \mathrm{ft} \text {. Depth of 1-in. } \\
\text { well }=42 \mathrm{ft} \text {. Sample collected by using a } \\
\text { low-flow bladder pump positioned at } \\
37 \mathrm{ft} \text { after purging of } 15 \mathrm{~L} \text {. }\end{array}$ \\
\hline $9 / 21 / 10$ & $14: 16$ & CNPMP6-W-27207 & MW & PMP6 & $50-60$ & 2716 & $9 / 21 / 10$ & $\begin{array}{l}\text { Depth to water }=20.00 \mathrm{ft} \text {. Depth of } 0.5 \text {-in. } \\
\text { well }=60 \mathrm{ft} \text {. Sample collected by using a } \\
\text { low-flow bladder pump positioned at } \\
55 \mathrm{ft} \text { after purging of } 7 \mathrm{~L} \text {. }\end{array}$ \\
\hline $9 / 21 / 10$ & $15: 32$ & CNQCTB-W-27217 b & TB & QC & - & 2716 & $9 / 21 / 10$ & $\begin{array}{l}\text { Trip blank sent to the AGEM Laboratory for } \\
\text { organic analysis with water samples } \\
\text { listed on COCs } 2717 \text { and } 2716 \text {. }\end{array}$ \\
\hline $9 / 21 / 10$ & $15: 40$ & CNDIH2O-W-27215 & FB & QC & - & 2716 & $9 / 21 / 10$ & $\begin{array}{l}\text { Blank of water used for equipment } \\
\text { decontamination. }\end{array}$ \\
\hline
\end{tabular}

a Sample types: CPT/P, piezometer; FB, field blank; MW, monitoring well; RI, rinsate; TB, trip blank.

b Quality control sample. 


\section{Appendix B:}

\section{Quality Control Data Summary}


TABLE B.1 Analytical results from the AGEM Laboratory for quality control samples collected in 2010 .

\begin{tabular}{|c|c|c|c|c|c|c|c|}
\hline \multirow[b]{2}{*}{ Location } & \multirow[b]{2}{*}{ Sample } & \multirow[b]{2}{*}{$\begin{array}{c}\text { Sample } \\
\text { Date }\end{array}$} & \multirow[b]{2}{*}{$\begin{array}{c}\text { Depth } \\
\text { (ft BGL) }\end{array}$} & \multicolumn{3}{|c|}{ Concentration $(\mu \mathrm{g} / \mathrm{L})$} & \multirow[b]{2}{*}{ Analysis Type } \\
\hline & & & & $\begin{array}{c}\text { Carbon } \\
\text { Tetrachloride }\end{array}$ & Chloroform & $\begin{array}{l}\text { Methylene } \\
\text { Chloride }\end{array}$ & \\
\hline PMP8 & CNPMP8-W-27183 & $4 / 5 / 10$ & $50-60$ & $0.4 \mathrm{Ja}$ & $0.7 \mathrm{~J}$ & $N D^{b}$ & Primary sample \\
\hline PMP8 & CNPMP8-W-27183DUP & $4 / 5 / 10$ & $50-60$ & $0.4 \mathrm{~J}$ & $0.7 \mathrm{~J}$ & ND & Duplicate analysis \\
\hline QC & CNQCIR-W-27184 & $4 / 5 / 10$ & - & ND & ND & ND & Equipment rinsate \\
\hline QC & CNQCTB-W-27185 & $4 / 5 / 10$ & - & ND & ND & ND & Trip blank \\
\hline MW03 & CNMW03-W-27188 & 9/19/10 & $50.5-60.5$ & 7.5 & $0.3 \mathrm{~J}$ & ND & Primary sample \\
\hline MW03 & CNMW03DUP-W-27211 & $9 / 19 / 10$ & $50.5-60.5$ & 7.8 & $0.3 \mathrm{~J}$ & ND & Replicate \\
\hline MW09 & CNMW09-W-27194 & 9/19/10 & $25-35$ & ND & ND & ND & Primary sample \\
\hline MW09 & CNMW09DUP-W-27212 & $9 / 19 / 10$ & $25-35$ & ND & ND & ND & Replicate \\
\hline SB07R & CNSB07R-W-27199 & $9 / 20 / 10$ & $45-60$ & 42 & 2.5 & ND & Primary sample \\
\hline SB07R & CNSB07R-W-27199DUP & $9 / 20 / 10$ & $45-60$ & 41 & 2.4 & ND & Duplicate analysis \\
\hline QC & CNQCIR-W-27213 & $9 / 20 / 10$ & - & ND & ND & ND & Equipment rinsate \\
\hline QC & CNQCIR-W-27214 & $9 / 20 / 10$ & - & ND & ND & ND & Equipment rinsate \\
\hline QC & CNQCTB-W-27216 & $9 / 20 / 10$ & - & ND & ND & ND & Trip blank \\
\hline PMP4 & CNPMP4-W-27205 & $9 / 21 / 10$ & $48.75-58.75$ & 28 & 1.8 & ND & Primary sample \\
\hline PMP4 & CNPMP4-W-27205DUP & $9 / 21 / 10$ & $48.75-58.75$ & 29 & 1.7 & ND & Duplicate analysis \\
\hline QC & CNDIH2O-W-27215 & $9 / 21 / 10$ & - & ND & ND & ND & Field blank \\
\hline QC & CNQCTB-W-27217 & $9 / 21 / 10$ & - & ND & ND & ND & Trip blank \\
\hline
\end{tabular}

a Qualifier J indicates an estimated concentration below the purge-and-trap method quantitation limit of $1.0 \mu \mathrm{g} / \mathrm{L}$.

b ND, not detected at an instrument detection limit of $0.1 \mu \mathrm{g} / \mathrm{L}$. 
TABLE B.2 Analytical results for verification groundwater samples analyzed at the AGEM Laboratrory and by TestAmerica.

\begin{tabular}{|c|c|c|c|c|c|c|c|c|c|}
\hline \multirow[b]{3}{*}{ Location } & \multirow[b]{3}{*}{ Sample } & \multirow[b]{3}{*}{$\begin{array}{c}\text { Sample } \\
\text { Date }\end{array}$} & \multirow[b]{3}{*}{$\begin{array}{l}\text { Depth } \\
\text { (ft BGL) }\end{array}$} & \multicolumn{6}{|c|}{ Concentration ( $\mu \mathrm{g} / \mathrm{L})$} \\
\hline & & & & \multicolumn{3}{|c|}{ AGEM Laborarory } & \multicolumn{3}{|c|}{ TestAmerica } \\
\hline & & & & $\begin{array}{c}\text { Carbon } \\
\text { Tetrachloride }\end{array}$ & Chloroform & $\begin{array}{l}\text { Methylene } \\
\text { Chloride }\end{array}$ & $\begin{array}{c}\text { Carbon } \\
\text { Tetrachloride }\end{array}$ & Chloroform & $\begin{array}{c}\text { Methylene } \\
\text { Chloride }\end{array}$ \\
\hline MW02 & CNMW02-W-27179 & $4 / 5 / 10$ & $49.5-59.5$ & $N D^{a}$ & ND & ND & ND & ND & ND \\
\hline PMP2 & CNPMP2-W-27181 & $4 / 5 / 10$ & $50-60$ & 991 & 182 & 5.1 & 670 & 130 & 4.3 \\
\hline PMP3 & CNPMP3-W-27182 & $4 / 5 / 10$ & $50-60$ & ND & ND & ND & ND & ND & ND \\
\hline MW06 & CNMW06-W-27191 & $9 / 20 / 10$ & $46.5-56.5$ & ND & ND & ND & $0.15 \mathrm{~J}^{\mathrm{b}}$ & ND & ND \\
\hline MW07 & CNMW07-W-27192 & $9 / 20 / 10$ & $45-55$ & 6.6 & $0.3 \mathrm{~J}$ & ND & 5.2 & ND & ND \\
\hline MW08 & CNMW08-W-27193 & $9 / 19 / 10$ & $38-53$ & ND & ND & ND & ND & ND & ND \\
\hline SB09 & CNSB09-W-27201 & $9 / 19 / 10$ & $32-42$ & ND & ND & ND & ND & ND & ND \\
\hline
\end{tabular}

a ND, not detected at an instrument detection limit of $0.1 \mu \mathrm{g} / \mathrm{L}$.

b Qualifier J indicates an estimated concentration below the method quantitation limit of $1.0 \mu \mathrm{g} / \mathrm{L}$ for analyses at the AGEM Laboratory or $0.5 \mu \mathrm{g} / \mathrm{L}$ for analyses by TestAmerica. 


\section{Appendix C:}

Time Series Diagrams for Selected Parameters at IM Monitoring Points 

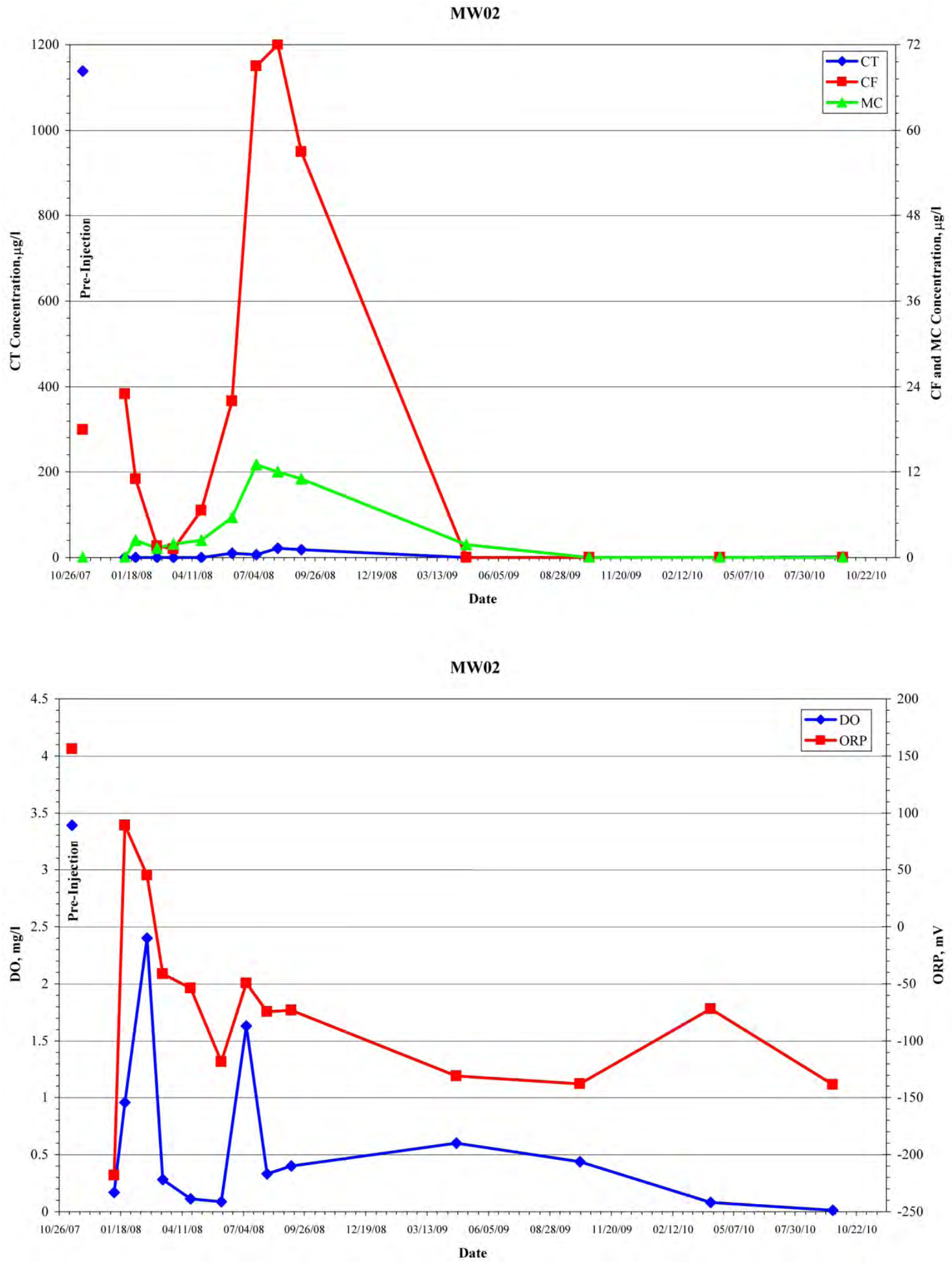

FIGURE C.1 Analytical results for VOCs, DO, and ORP in groundwater samples collected at location MW02, November 2007 to September 2010. 

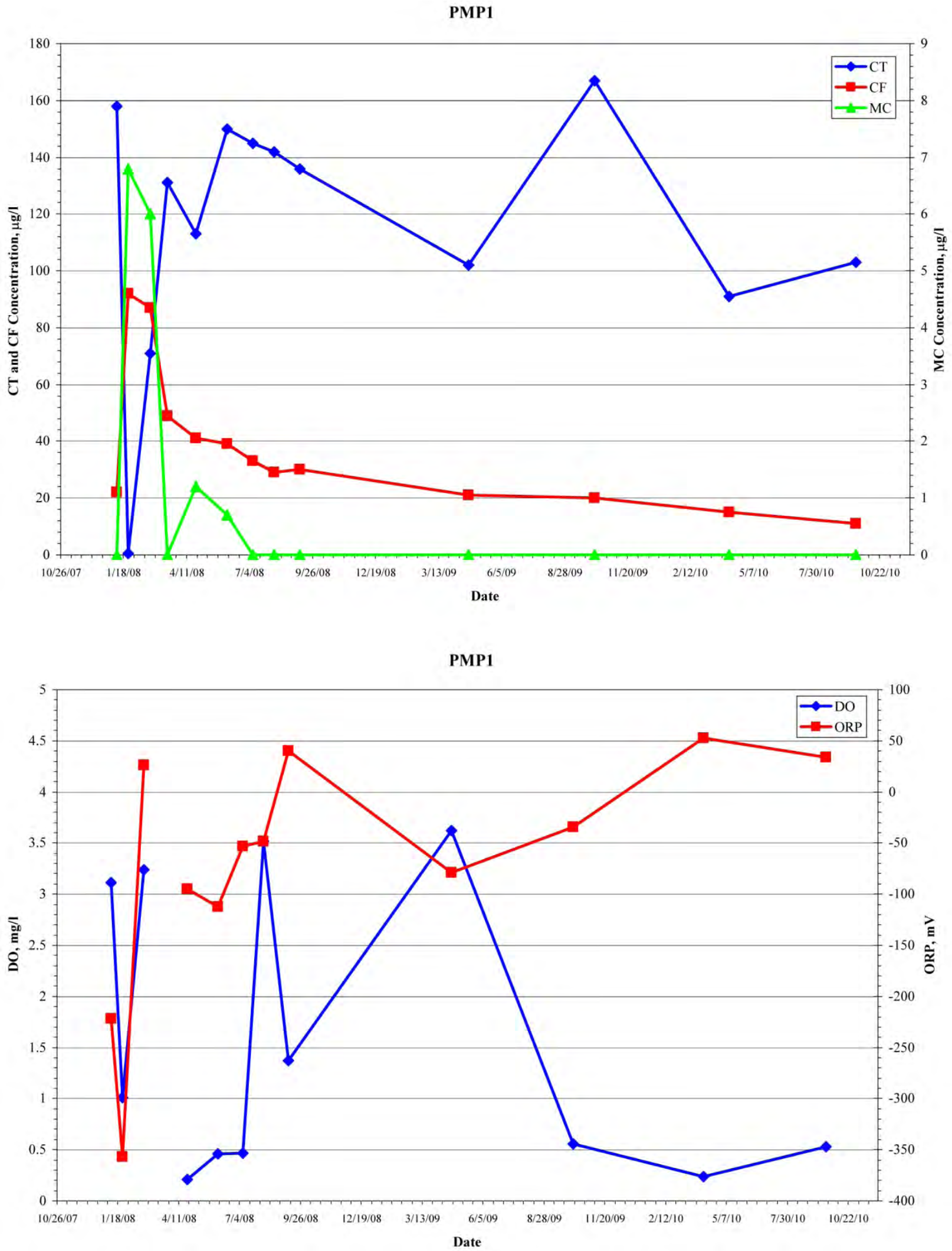

FIGURE C.2 Analytical results for VOCs, DO, and ORP in groundwater samples collected at location PMP1, January 2008 to September 2010. 
PSB2-PMP2

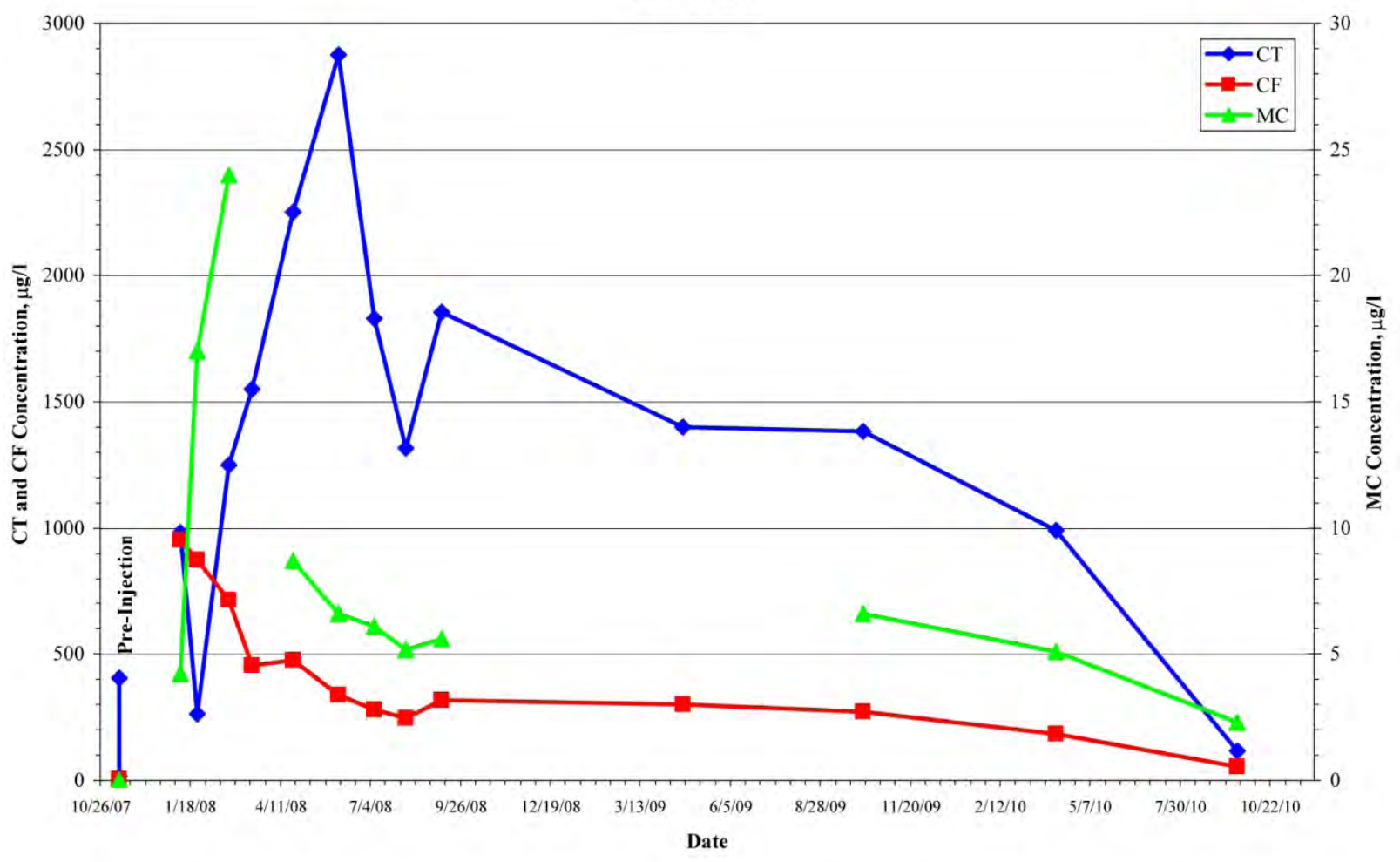

PSB2-PMP2

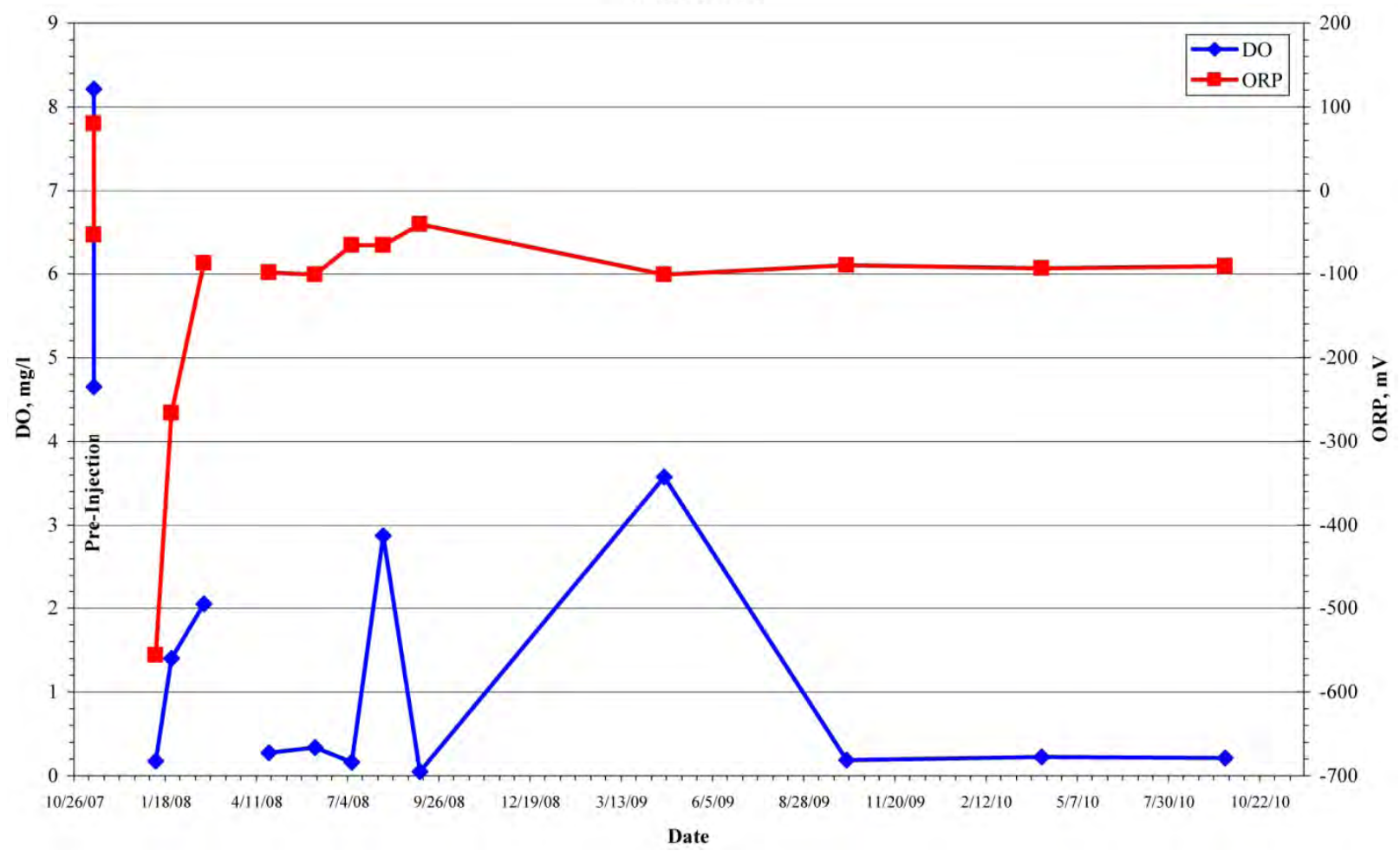

FIGURE C.3 Analytical results for VOCs, DO, and ORP in groundwater samples collected at locations PSB2 and PMP2, November 2007 to September 2010. 
PSB3-PMP3
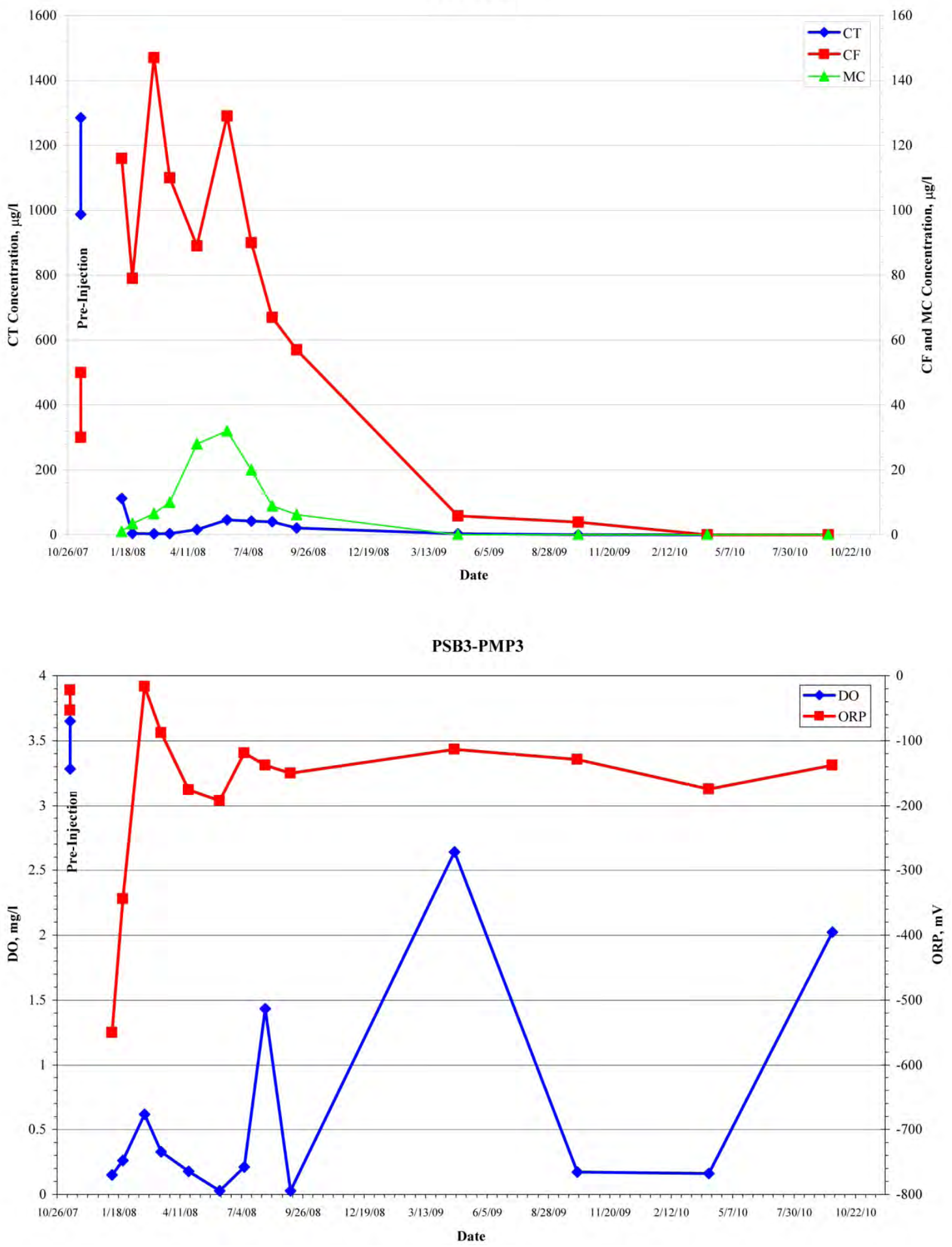

FIGURE C.4 Analytical results for VOCs, DO, and ORP in groundwater samples collected at locations PSB3 and PMP3, November 2007 to September 2010. 
PMP4

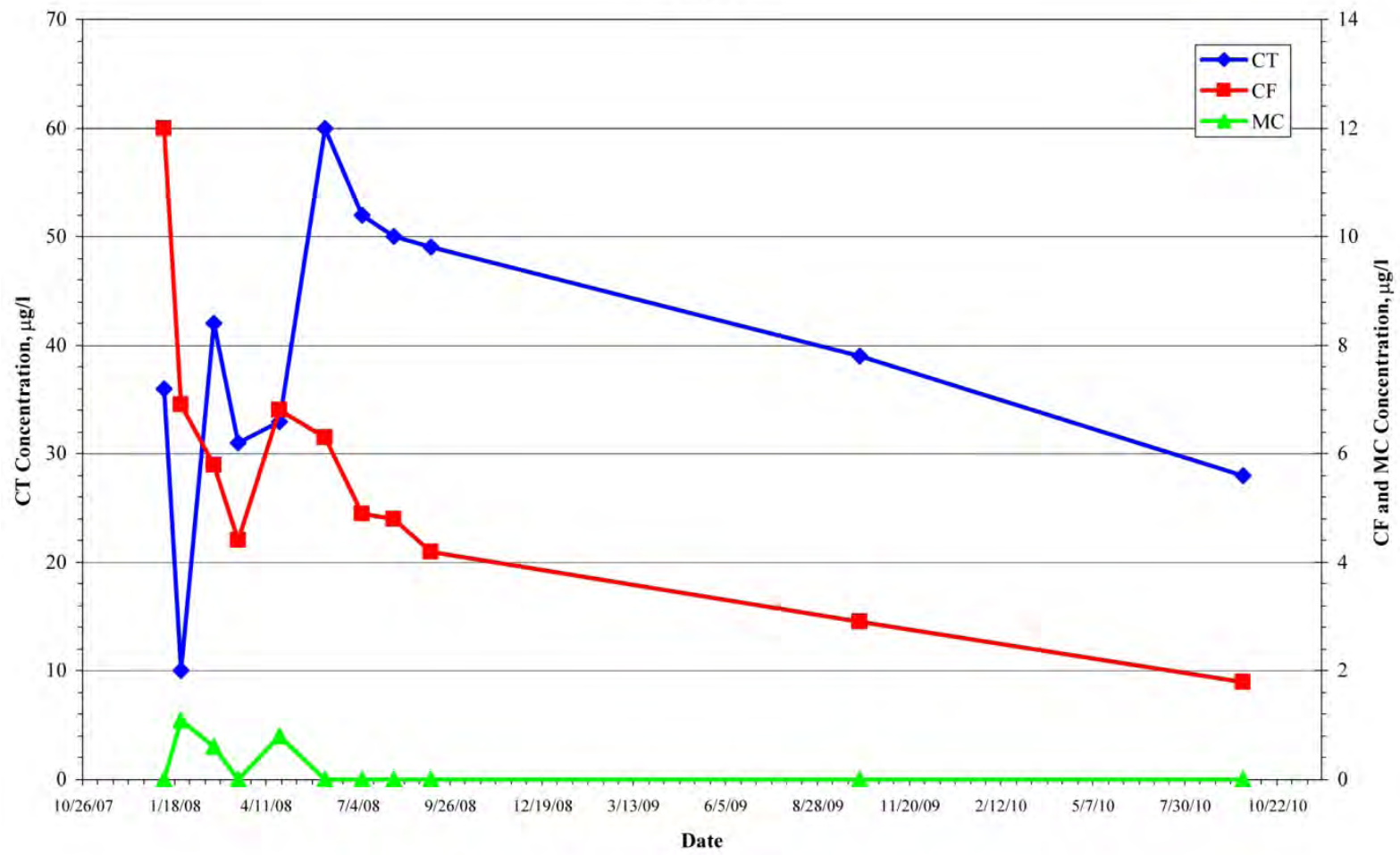

PMP4

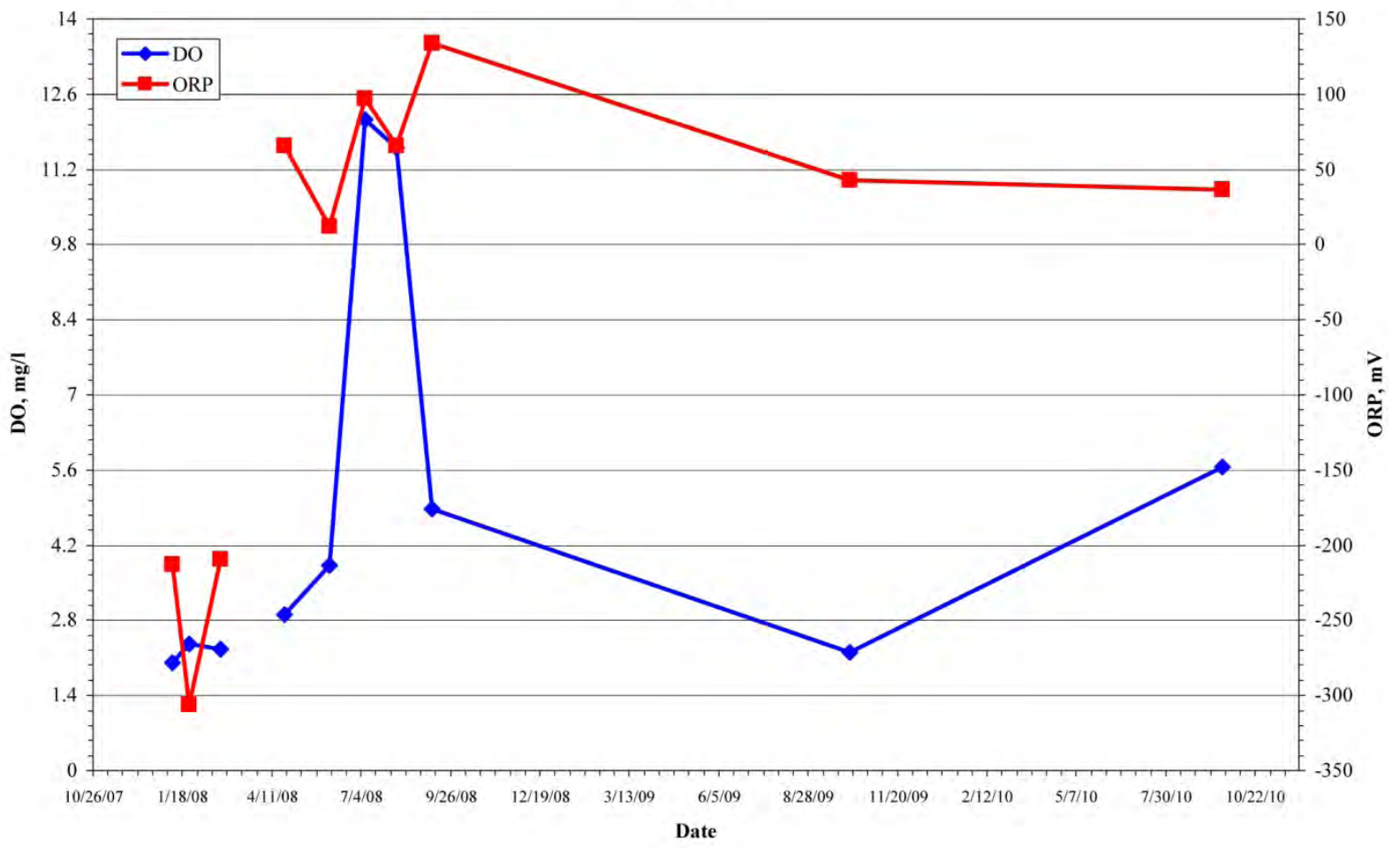

FIGURE C.5 Analytical results for VOCs, DO, and ORP in groundwater samples collected at location PMP4, January 2008 to September 2010. 
PSB5-PMP5

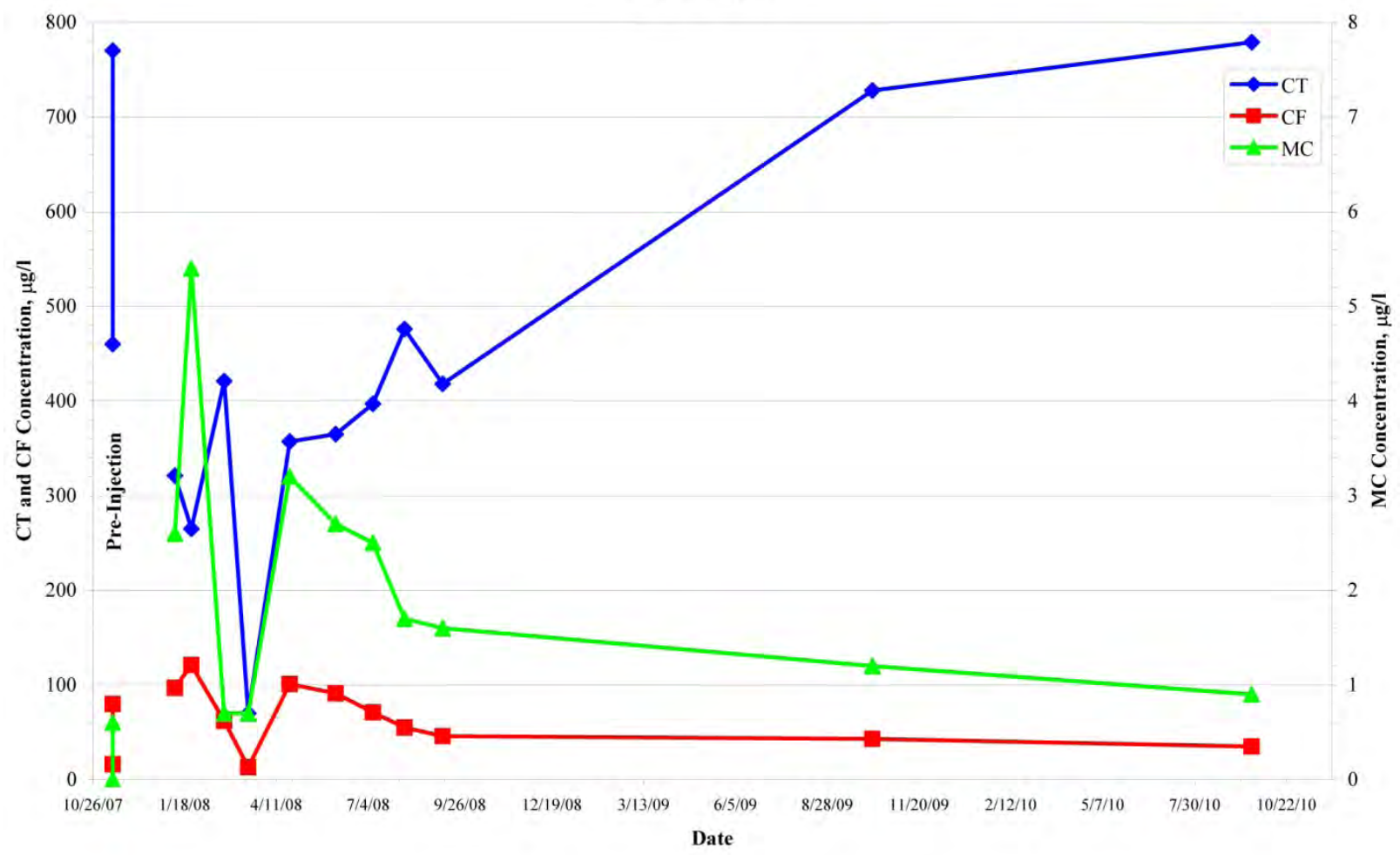

PSB5-PMP5

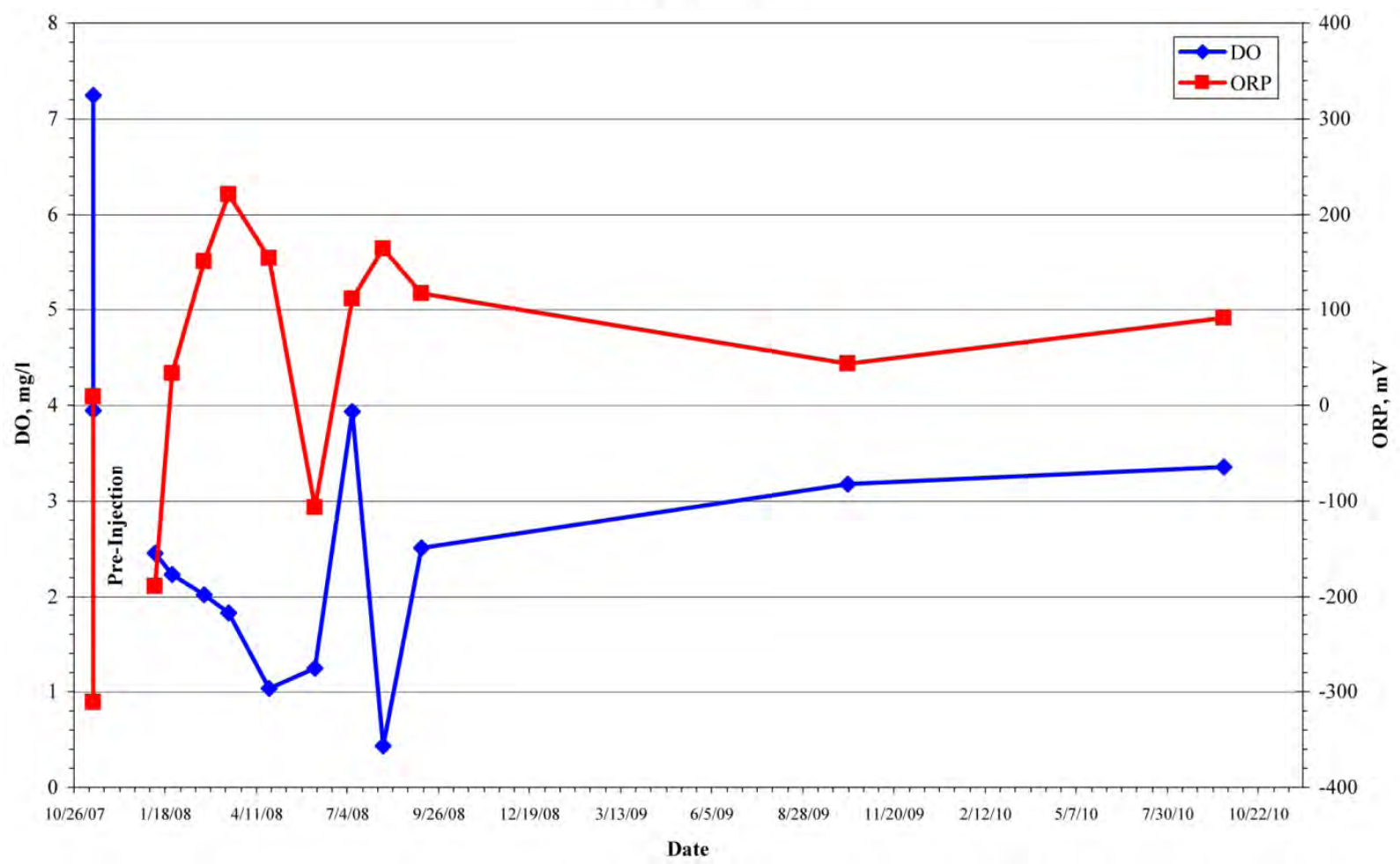

FIGURE C.6 Analytical results for VOCs, DO, and ORP in groundwater samples collected at locations PSB5 and PMP5, November 2007 to September 2010. 


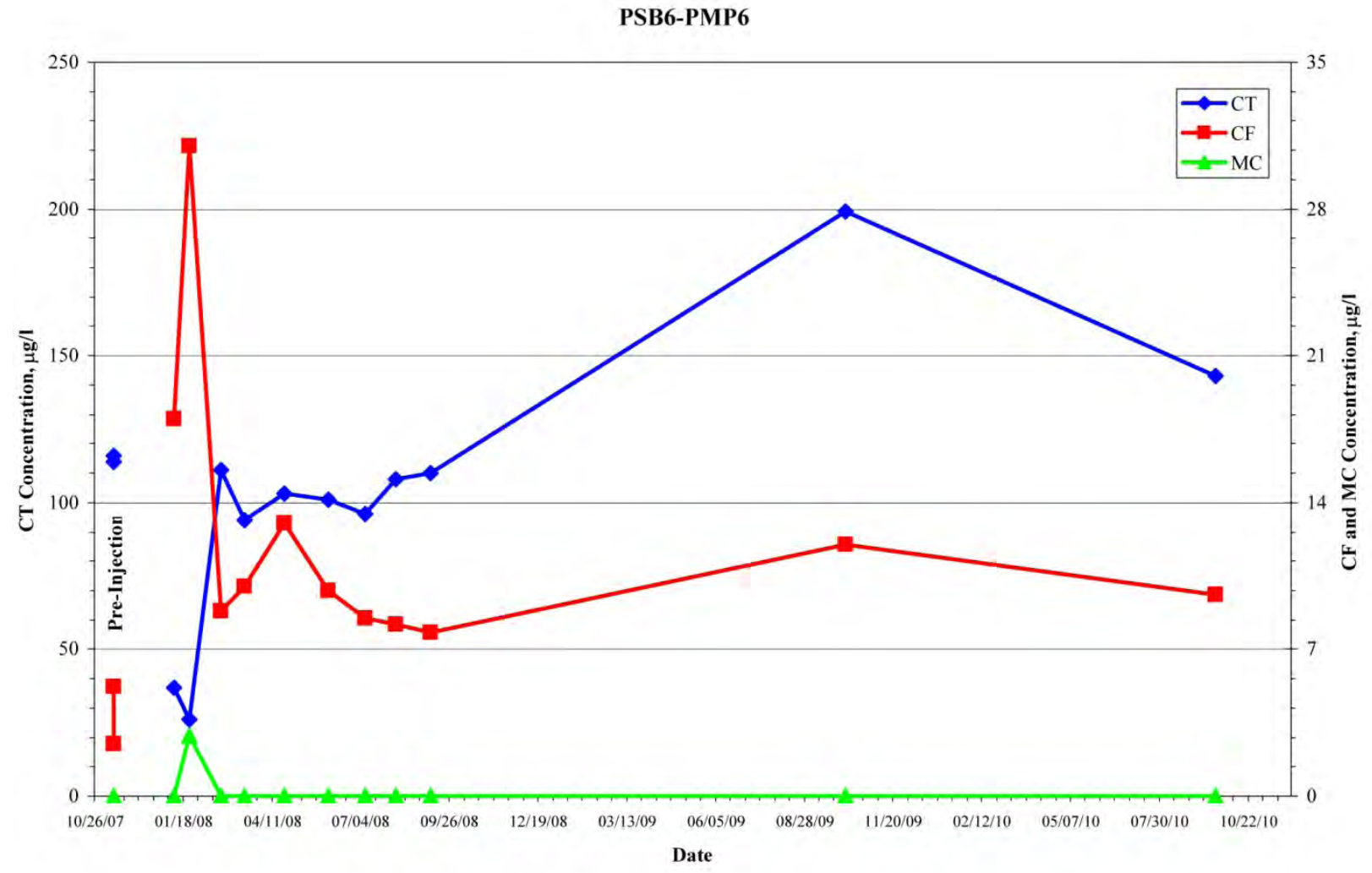

PSB6-PMP6

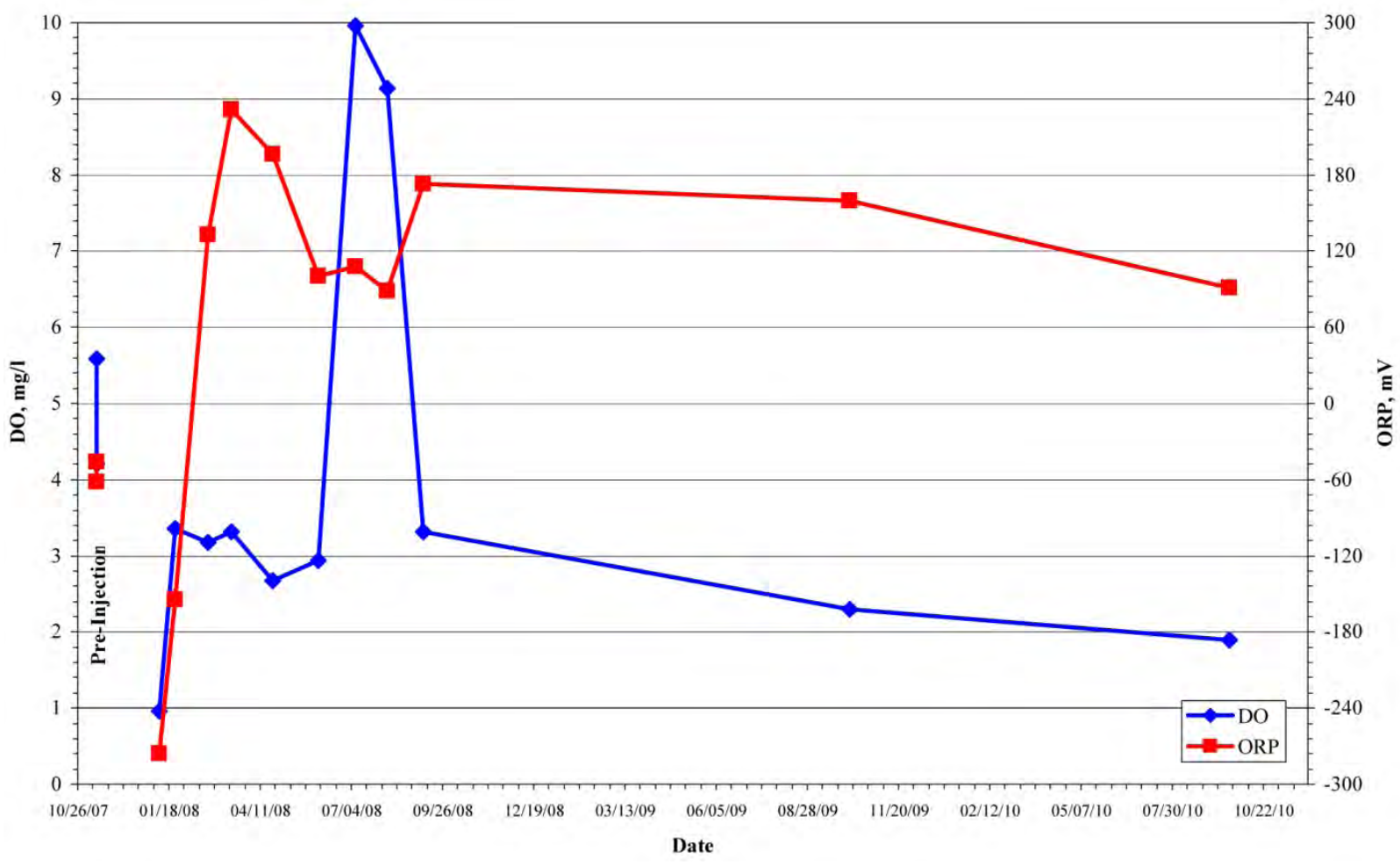

FIGURE C.7 Analytical results for VOCs, DO, and ORP in groundwater samples collected at locations PSB6 and PMP6, November 2007 to September 2010. 

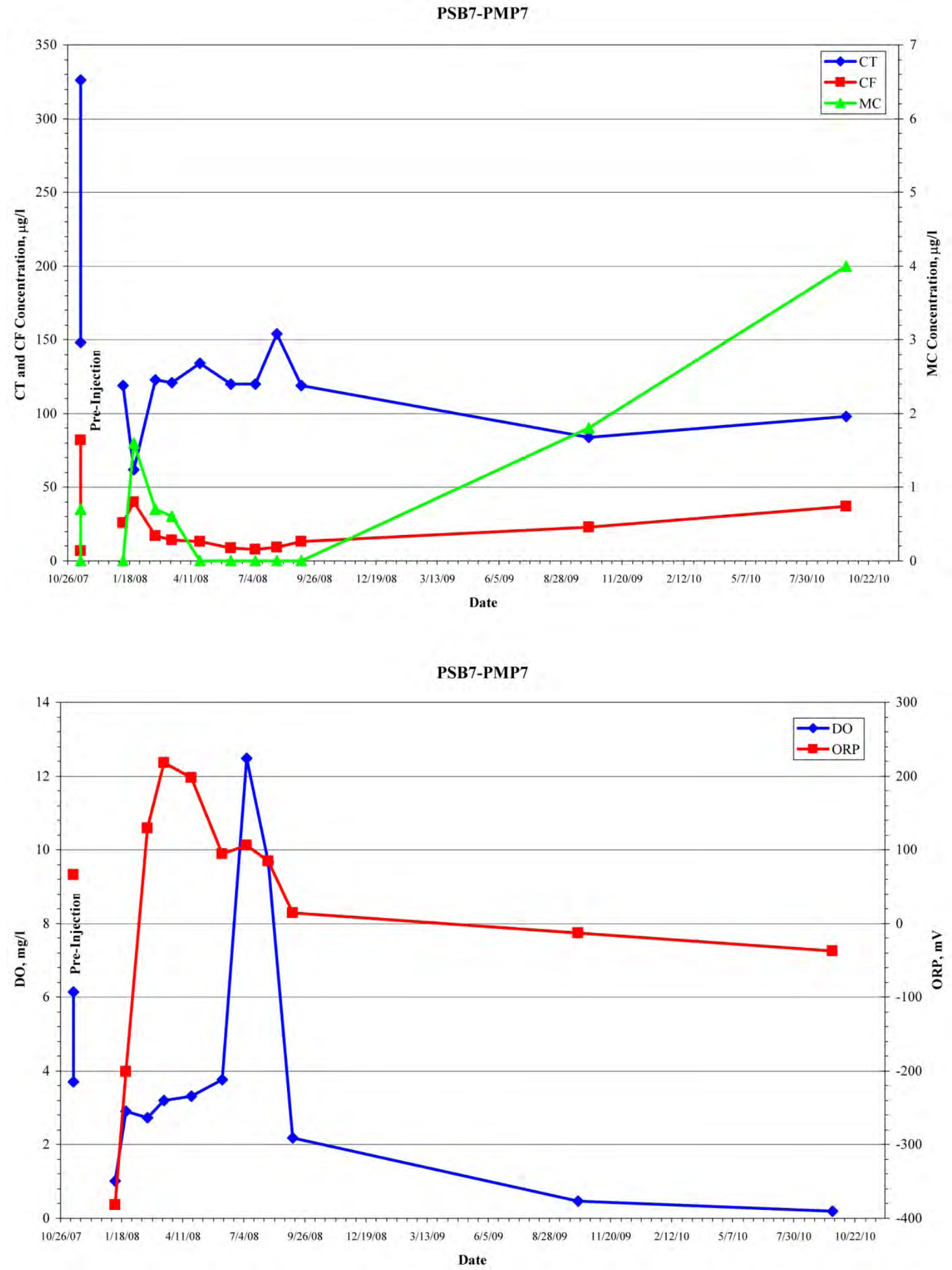

FIGURE C.8 Analytical results for VOCs, DO, and ORP in groundwater samples collected at locations PSB7 and PMP7, November 2007 to September 2010. 
PSB8-PMP8

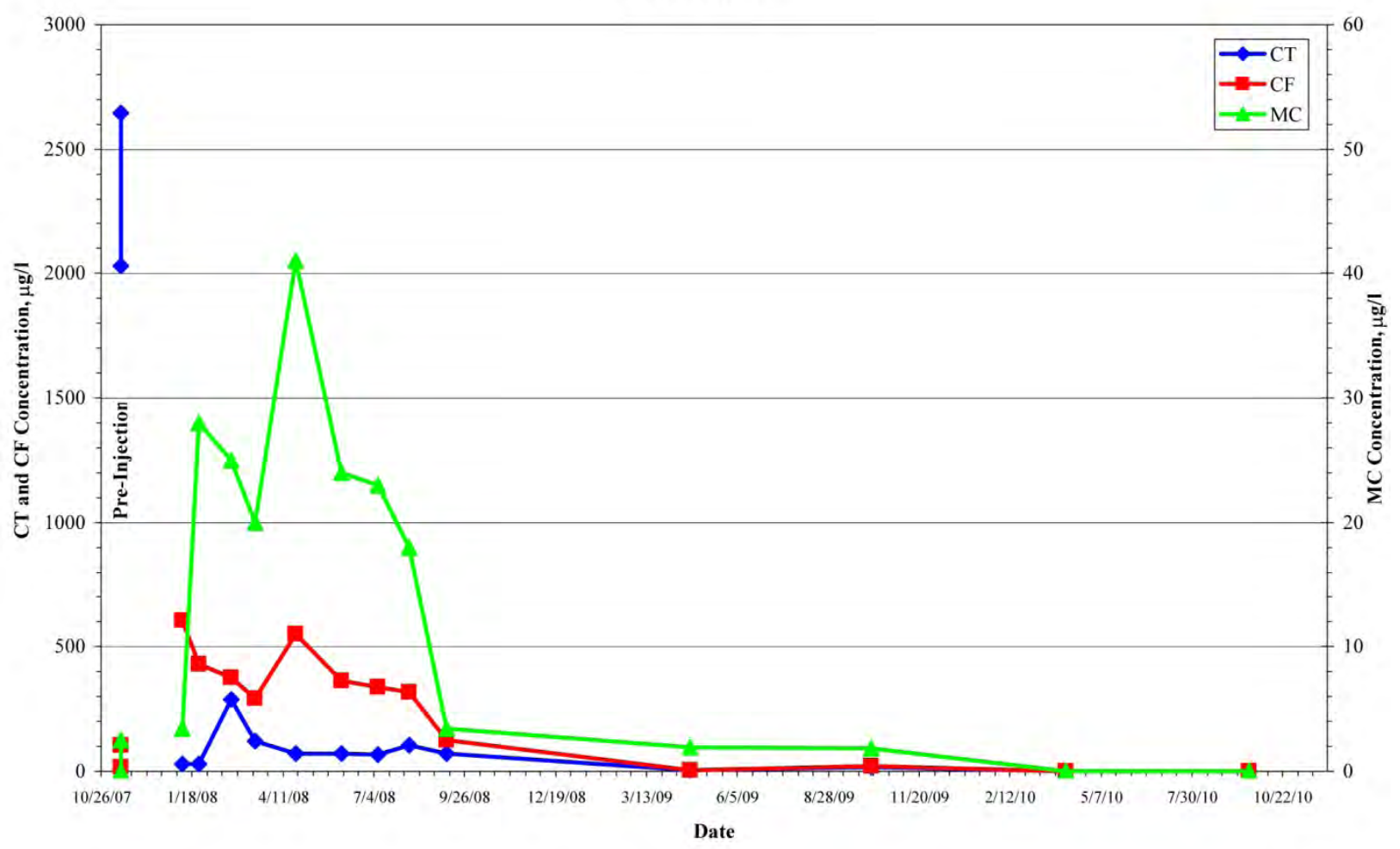

PSB8-PMP8

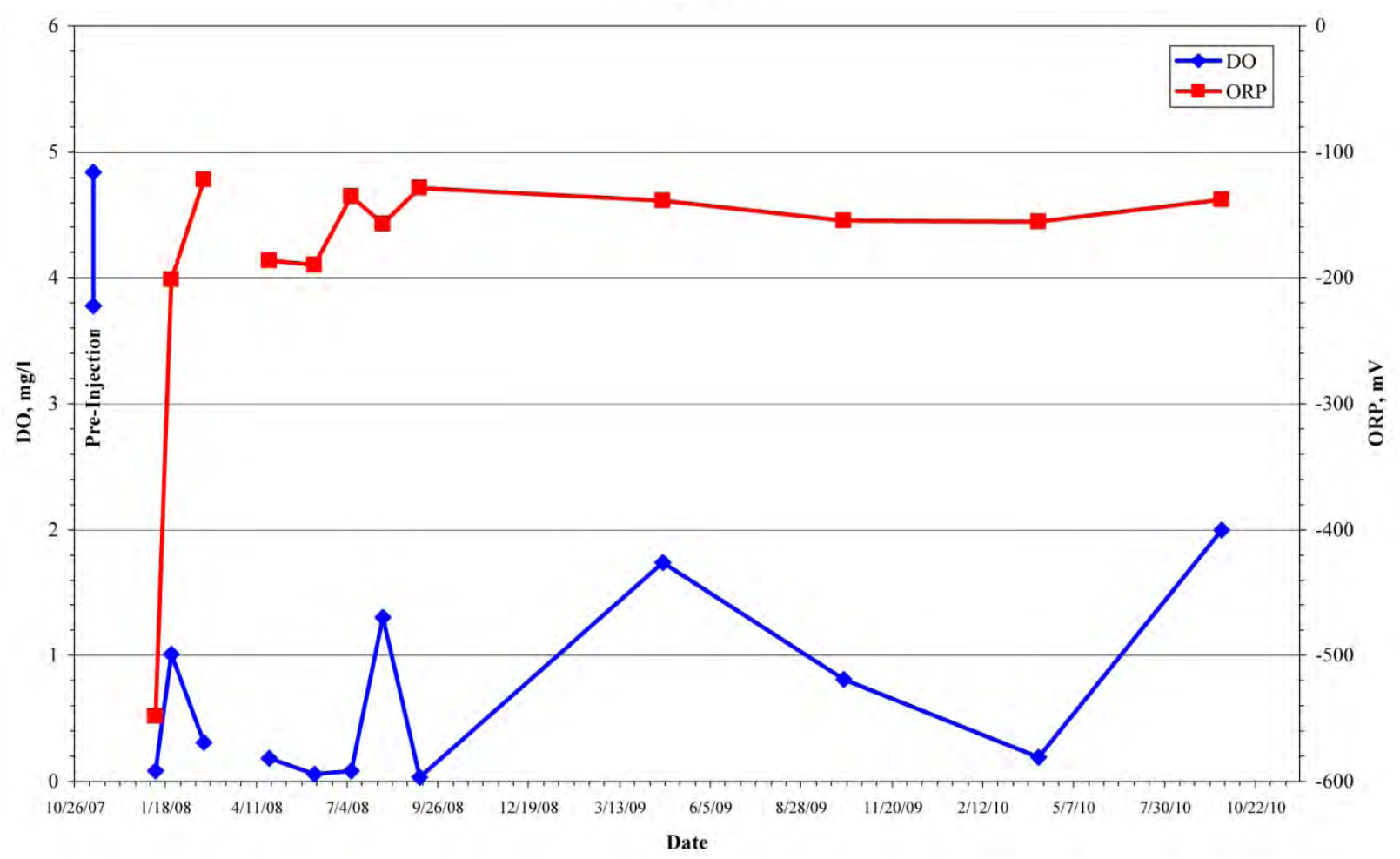

FIGURE C.9 Analytical results for VOCs, DO, and ORP in groundwater samples collected at locations PSB8 and PMP8, November 2007 to September 2010. 
PSB9-PMP9

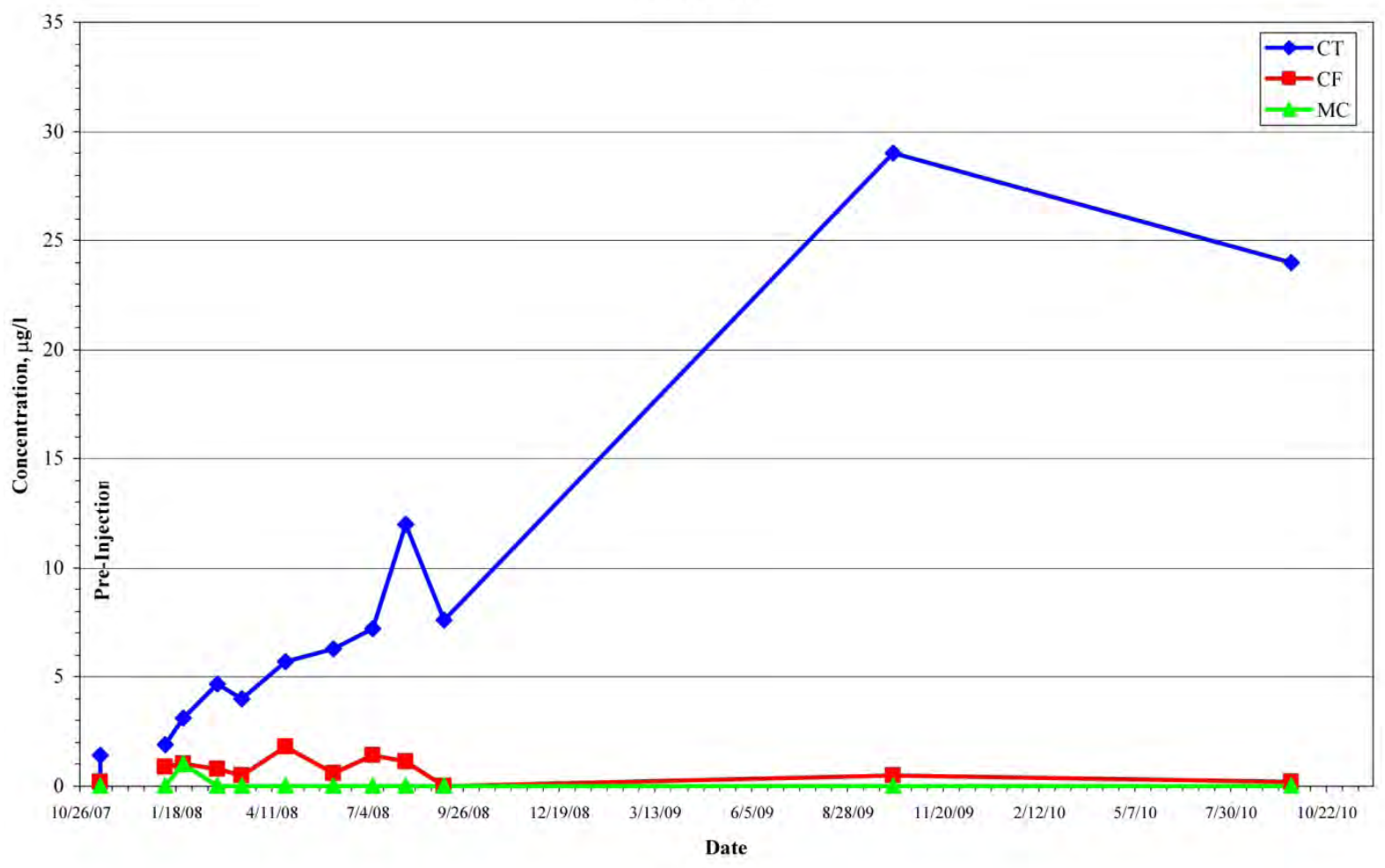

PSB9-PMP9

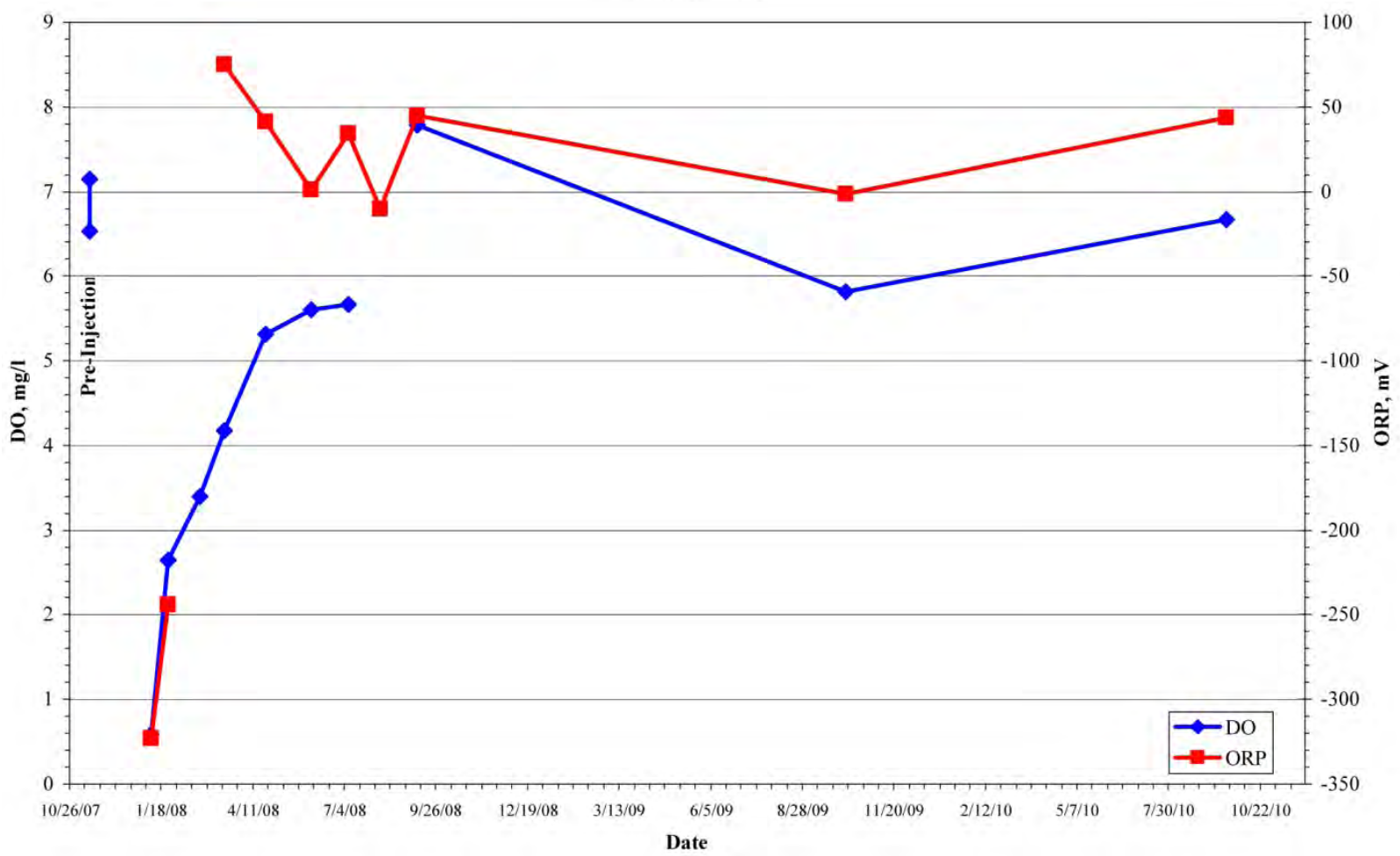

FIGURE C.10 Analytical results for VOCs, DO, and ORP in groundwater samples collected at locations PSB9 and PMP9, November 2007 to September 2010. 


\section{Supplement 1:}

\section{Waste Characterization and Disposal Documentation}


October 14, 2010

Mr. Travis Kamler

TCW Construction Inc

$141 \mathrm{M}$ Street

Lincoln, NE 68508

RE: Project: Kansas Waste Water

Pace Project No.: 6086606

Dear Mr. Kamler:

Enclosed are the analytical results for sample(s) received by the laboratory on October 01, 2010.

The results relate only to the samples included in this report. Results reported herein conform to the most current NELAC standards, where applicable, unless otherwise narrated in the body of the report.

If you have any questions concerning this report, please feel free to contact me.

Sincerely,

$$
\text { sudy sigoon }
$$

Trudy Gipson

trudy.gipson@pacelabs.com

Project Manager

Enclosures

cc: Mr. David Surgnier 


\section{CERTIFICATIONS}

Project:

Kansas Waste Water

Pace Project No.: 6086606

Kansas Certification IDs

9608 Loiret Boulevard, Lenexa, KS 66219

A2LA Certification \#: 2456.01

Arkansas Certification \#: 05-008-0

Illinois Certification \#: 001191

lowa Certification \#: 118

Kansas/NELAP Certification \#: E-10116

Louisiana Certification \#: 03055

Nevada Certification \#: KS000212008A

Oklahoma Certification \#: 9205/9935

Texas Certification \#: T104704407-08-TX

Utah Certification \#: 9135995665

This report shall not be reproduced, except in full, without the written consent of Pace Analytical Services, Inc. 
SAMPLE SUMMARY

\begin{tabular}{|c|c|c|c|c|}
\hline Project: & Kansas Waste Water & & & \\
\hline Lab ID & Sample ID & Matrix & Date Collected & Date Received \\
\hline 6086606001 & BAPURGE-W-930101 & Water & 09/30/10 09:00 & 10/01/10 09:15 \\
\hline 6086606002 & CNPURGE-W-930102 & Water & 09/30/10 10:00 & 10/01/10 09:15 \\
\hline 6086606003 & EVPURGE-W-930103 & Water & 09/30/10 11:32 & 10/01/10 09:15 \\
\hline 6086606004 & MRPURGE-W-930104 & Water & 09/30/10 13:42 & 10/01/10 09:15 \\
\hline
\end{tabular}


SAMPLE ANALYTE COUNT

Project: Kansas Waste Water

Pace Project No.: 6086606

\begin{tabular}{|c|c|c|c|c|}
\hline Lab ID & Sample ID & Method & Analysts & $\begin{array}{l}\text { Analytes } \\
\text { Reported }\end{array}$ \\
\hline \multirow[t]{3}{*}{6086606001} & BAPURGE-W-930101 & EPA 504.1 & NAW & 1 \\
\hline & & EPA 5030B/8260 & HMW & 70 \\
\hline & & EPA 300.0 & RAB & 1 \\
\hline \multirow[t]{3}{*}{6086606002} & CNPURGE-W-930102 & EPA 504.1 & NAW & 1 \\
\hline & & EPA $5030 B / 8260$ & HMW & 70 \\
\hline & & EPA 300.0 & $\mathrm{RAB}$ & 1 \\
\hline \multirow[t]{3}{*}{6086606003} & EVPURGE-W-930103 & EPA 504.1 & NAW & 1 \\
\hline & & EPA 5030B/8260 & HMW & 70 \\
\hline & & EPA 300.0 & $\mathrm{RAB}$ & 1 \\
\hline \multirow[t]{3}{*}{6086606004} & MRPURGE-W-930104 & EPA 504.1 & NAW & 1 \\
\hline & & EPA 5030B/8260 & HMW & 70 \\
\hline & & EPA 300.0 & $\mathrm{RAB}$ & 1 \\
\hline
\end{tabular}




\section{ANALYTICAL RESULTS}

Project: Kansas Waste Water

Pace Project No.: 6086606

Sample: BAPURGE-W-930101 Lab ID: 6086606001 Collected: 09/30/10 09:00 Received: 10/01/10 09:15 Matrix: Water

\begin{tabular}{|c|c|}
\hline Parameters & Results \\
\hline
\end{tabular}

504 GCS EDB and DBCP

1,2-Dibromoethane (EDB)

\section{MSV}

Acetone

Benzene

Bromobenzene

Bromochloromethane

Bromodichloromethane

Bromoform

Bromomethane

2-Butanone (MEK)

n-Butylbenzene

sec-Butylbenzene

tert-Butylbenzene

Carbon disulfide

Carbon tetrachloride

Chlorobenzene

Chloroethane

Chloroform

Chloromethane

2-Chlorotoluene

4-Chlorotoluene

1,2-Dibromo-3-chloropropane

Dibromochloromethane

1,2-Dibromoethane (EDB)

Dibromomethane

1,2-Dichlorobenzene

1,3-Dichlorobenzene

1,4-Dichlorobenzene

Dichlorodifluoromethane

1,1-Dichloroethane

1,2-Dichloroethane

1,2-Dichloroethene (Total)

1,1-Dichloroethene

cis-1,2-Dichloroethene

trans-1,2-Dichloroethene

1,2-Dichloropropane

1,3-Dichloropropane

2,2-Dichloropropane

1,1-Dichloropropene

cis-1,3-Dichloropropene

trans-1,3-Dichloropropene

Ethylbenzene

Hexachloro-1,3-butadiene

2-Hexanone

Isopropylbenzene (Cumene)

p-Isopropyltoluene

Date: 10/14/2010 11:06 AM

Analytical Method: EPA 504.1 Preparation Method: EPA 504.1

$$
\begin{array}{llllllll}
\text { ND ug/L } & 0.029 & 1 & 10 / 07 / 10 & 00: 00 & 10 / 07 / 10 & 21: 23 & 106-93-4
\end{array}
$$

Analytical Method: EPA 5030B/8260

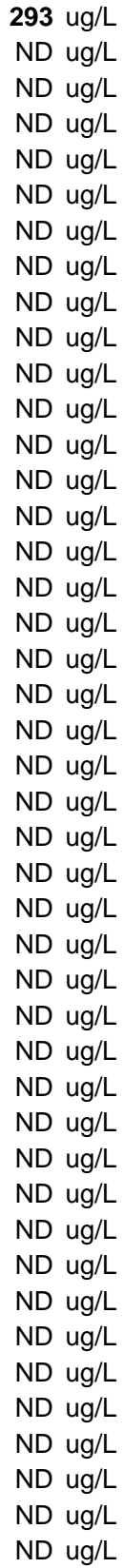

\begin{tabular}{rr}
10.0 & 1 \\
1.0 & 1 \\
1.0 & 1 \\
1.0 & 1 \\
1.0 & 1 \\
1.0 & 1 \\
1.0 & 1 \\
10.0 & 1 \\
1.0 & 1 \\
1.0 & 1 \\
1.0 & 1 \\
5.0 & 1 \\
1.0 & 1 \\
1.0 & 1 \\
1.0 & 1 \\
1.0 & 1 \\
1.0 & 1 \\
1.0 & 1 \\
1.0 & 1 \\
2.5 & 1 \\
1.0 & 1 \\
1.0 & 1 \\
1.0 & 1 \\
1.0 & 1 \\
1.0 & 1 \\
1.0 & 1 \\
1.0 & 1 \\
1.0 & 1 \\
1.0 & 1 \\
1.0 & 1 \\
1.0 & 1 \\
1.0 & 1 \\
1.0 & 1 \\
1.0 & 1 \\
1.0 & 1 \\
1.0 & 1 \\
1.0 & 1 \\
1.0 & 1 \\
1.0 & 1 \\
1.0 & 1 \\
1.0 & 1 \\
10.0 & 1 \\
1.0 & 1 \\
1.0 & 1 \\
& \\
\hline
\end{tabular}

$10 / 03 / 10$ 12:46 67-64-1 $10 / 03 / 1012: 46 \quad 71-43-2$ $10 / 03 / 1012: 46 \quad 108-86-1$ $10 / 03 / 1012: 46 \quad 74-97-5$ $10 / 03 / 1012: 46 \quad 75-27-4$ $10 / 03 / 1012: 46 \quad 75-25-2$ $10 / 03 / 1012: 46 \quad 74-83-9$ $10 / 03 / 1012: 46 \quad 78-93-3$ $10 / 03 / 1012: 46 \quad 104-51-8$ $10 / 03 / 1012: 46 \quad 135-98-8$ $10 / 03 / 1012: 46$ 98-06-6 $10 / 03 / 1012: 46 \quad 75-15-0$ $10 / 03 / 1012: 46 \quad 56-23-5$ $10 / 03 / 1012: 46 \quad 108-90-7$ $10 / 03 / 1012: 46 \quad 75-00-3$ 10/03/10 12:46 67-66-3 10/03/10 12:46 74-87-3 $10 / 03 / 1012: 46 \quad 95-49-8$ $10 / 03 / 10$ 12:46 106-43-4 $10 / 03 / 1012: 46 \quad 96-12-8$ $10 / 03 / 10 \quad 12: 46 \quad 124-48-1$ $10 / 03 / 10$ 12:46 106-93-4 10/03/10 12:46 74-95-3 10/03/10 12:46 95-50-1 10/03/10 12:46 541-73-1 10/03/10 12:46 106-46-7 10/03/10 12:46 75-71-8 $10 / 03 / 10$ 12:46 75-34-3 $10 / 03 / 10 \quad 12: 46 \quad 107-06-2$ 10/03/10 12:46 540-59-0 $10 / 03 / 10$ 12:46 75-35-4 $10 / 03 / 1012: 46 \quad 156-59-2$ $10 / 03 / 1012: 46 \quad 156-60-5$ 10/03/10 12:46 78-87-5 $10 / 03 / 10 \quad 12: 46 \quad 142-28-9$ $10 / 03 / 1012: 46 \quad 594-20-7$ 10/03/10 12:46 563-58-6 10/03/10 12:46 10061-01-5 10/03/10 12:46 10061-02-6 $10 / 03 / 10 \quad 12: 46 \quad 100-41-4$ 10/03/10 12:46 87-68-3 10/03/10 12:46 591-78-6 $10 / 03 / 1012: 46 \quad 98-82-8$ $10 / 03 / 10$ 12:46 99-87-6

This report shall not be reproduced, except in full, without the written consent of Pace Analytical Services, Inc. 


\section{ANALYTICAL RESULTS}

Project: Kansas Waste Water

Pace Project No.: 6086606

Sample: BAPURGE-W-930101 Lab ID: 6086606001 $\quad$ Collected: 09/30/10 09:00 Received: 10/01/10 09:15 Matrix: Water

Parameters $\longrightarrow$ Results $\longrightarrow$ Units $\longrightarrow$ Report Limit $\longrightarrow$ DF

8260 MSV

Methylene chloride

4-Methyl-2-pentanone (MIBK)

Methyl-tert-butyl ether

Naphthalene

n-Propylbenzene

Styrene

1,1,1,2-Tetrachloroethane

1,1,2,2-Tetrachloroethane

Tetrachloroethene

Toluene

1,2,3-Trichlorobenzene

1,2,4-Trichlorobenzene

1,1,1-Trichloroethane

1,1,2-Trichloroethane

Trichloroethene

Trichlorofluoromethane

1,2,3-Trichloropropane

1,2,4-Trimethylbenzene

1,3,5-Trimethylbenzene

Vinyl chloride

Xylene (Total)

4-Bromofluorobenzene (S)

Dibromofluoromethane (S)

1,2-Dichloroethane-d4 (S)

Toluene-d8 (S)

Preservation $\mathrm{pH}$

\subsection{IC Anions}

Nitrate as $\mathrm{N}$
Analytical Method: EPA 5030B/8260

\begin{tabular}{|c|c|c|c|c|}
\hline ND ug/L & 1.0 & 1 & $10 / 03 / 1012: 46$ & $75-09-2$ \\
\hline ND ug/L & 10.0 & 1 & $10 / 03 / 1012: 46$ & $108-10-1$ \\
\hline ND ug/L & 1.0 & 1 & $10 / 03 / 1012: 46$ & $1634-04-4$ \\
\hline ND ug/L & 10.0 & 1 & $10 / 03 / 1012: 46$ & $91-20-3$ \\
\hline ND ug/L & 1.0 & 1 & $10 / 03 / 1012: 46$ & $103-65-1$ \\
\hline ND ug/L & 1.0 & 1 & $10 / 03 / 1012: 46$ & $100-42-5$ \\
\hline ND ug/L & 1.0 & 1 & $10 / 03 / 1012: 46$ & $630-20-6$ \\
\hline ND ug/L & 1.0 & 1 & $10 / 03 / 1012: 46$ & $79-34-5$ \\
\hline ND ug/L & 1.0 & 1 & $10 / 03 / 1012: 46$ & $127-18-4$ \\
\hline ND ug/L & 1.0 & 1 & $10 / 03 / 1012: 46$ & $108-88-3$ \\
\hline ND ug/L & 1.0 & 1 & $10 / 03 / 1012: 46$ & $87-61-6$ \\
\hline ND ug/L & 1.0 & 1 & $10 / 03 / 1012: 46$ & $120-82-1$ \\
\hline ND ug/L & 1.0 & 1 & $10 / 03 / 1012: 46$ & $71-55-6$ \\
\hline ND ug/L & 1.0 & 1 & $10 / 03 / 1012: 46$ & $79-00-5$ \\
\hline ND ug/L & 1.0 & 1 & $10 / 03 / 1012: 46$ & $79-01-6$ \\
\hline ND ug/L & 1.0 & 1 & $10 / 03 / 1012: 46$ & $75-69-4$ \\
\hline ND ug/L & 2.5 & 1 & $10 / 03 / 1012: 46$ & $96-18-4$ \\
\hline ND ug/L & 1.0 & 1 & $10 / 03 / 1012: 46$ & $95-63-6$ \\
\hline ND ug/L & 1.0 & 1 & $10 / 03 / 1012: 46$ & $108-67-8$ \\
\hline ND ug/L & 1.0 & 1 & $10 / 03 / 1012: 46$ & $75-01-4$ \\
\hline ND ug/L & 3.0 & 1 & $10 / 03 / 1012: 46$ & $1330-20-7$ \\
\hline $93 \%$ & $87-113$ & 1 & $10 / 03 / 1012: 46$ & $460-00-4$ \\
\hline $103 \%$ & $86-112$ & 1 & $10 / 03 / 1012: 46$ & $1868-53-7$ \\
\hline $98 \%$ & $82-119$ & 1 & $10 / 03 / 1012: 46$ & $17060-07-0$ \\
\hline $102 \%$ & $90-110$ & 1 & $10 / 03 / 1012: 46$ & $2037-26-5$ \\
\hline 7.0 & 0.10 & 1 & $10 / 03 / 1012: 46$ & \\
\hline
\end{tabular}

Analytical Method: EPA 300.0

$2.7 \mathrm{mg} / \mathrm{L}$

$0.10 \quad 1$

10/01/10 18:59 14797-55-8

This report shall not be reproduced, except in full, 


\section{ANALYTICAL RESULTS}

Project: Kansas Waste Water

Pace Project No.: 6086606

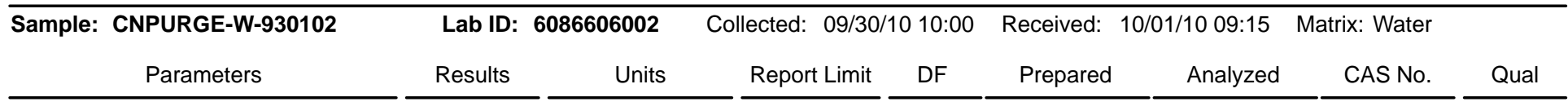

504 GCS EDB and DBCP

1,2-Dibromoethane (EDB)

\section{MSV}

Acetone

Benzene

Bromobenzene

Bromochloromethane

Bromodichloromethane

Bromoform

Bromomethane

2-Butanone (MEK)

n-Butylbenzene

sec-Butylbenzene

tert-Butylbenzene

Carbon disulfide

Carbon tetrachloride

Chlorobenzene

Chloroethane

Chloroform

Chloromethane

2-Chlorotoluene

4-Chlorotoluene

1,2-Dibromo-3-chloropropane

Dibromochloromethane

1,2-Dibromoethane (EDB)

Dibromomethane

1,2-Dichlorobenzene

1,3-Dichlorobenzene

1,4-Dichlorobenzene

Dichlorodifluoromethane

1,1-Dichloroethane

1,2-Dichloroethane

1,2-Dichloroethene (Total)

1,1-Dichloroethene

cis-1,2-Dichloroethene

trans-1,2-Dichloroethene

1,2-Dichloropropane

1,3-Dichloropropane

2,2-Dichloropropane

1,1-Dichloropropene

cis-1,3-Dichloropropene

trans-1,3-Dichloropropene

Ethylbenzene

Hexachloro-1,3-butadiene

2-Hexanone

Isopropylbenzene (Cumene)

p-Isopropyltoluene

Date: 10/14/2010 11:06 AM

Analytical Method: EPA 504.1 Preparation Method: EPA 504.1

$$
\begin{array}{llllllll}
\text { ND ug/L } & 0.029 & 1 & 10 / 07 / 10 & 00: 00 & 10 / 07 / 10 & 21: 34 & 106-93-4
\end{array}
$$

Analytical Method: EPA 5030B/8260

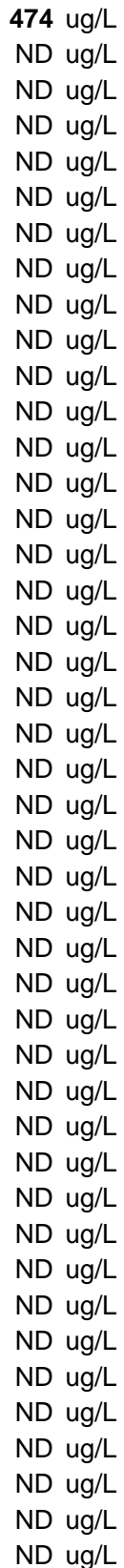

$474 \mathrm{ug} / \mathrm{L}$

ND ug/L

ND ug/L

ug/L

ND ug/L

ND ug/L

ND ug/L

ND ug/L

$N D$ ug/L

$N D$ ug/L

ND ug/L

ND $u g / L$

ug/L

ND ug/L

$\mathrm{ND}$ ug/L

ug/L

ND ug/L

ND ug/L

ND ug/L

ND ug/L

ND ug/L

D ug/L

ND ug/L

ND ug/L

ND ug/L

ND ug/L

$\mathrm{ND}$ ug/L

ND ug/L

ND ug/L

ND ug/L

ND ug/L

ND ug/L

ND ug/L

ND ug/L

$\begin{array}{rr}10.0 & 1 \\ 1.0 & 1 \\ 1.0 & 1 \\ 1.0 & 1 \\ 1.0 & 1 \\ 1.0 & 1 \\ 1.0 & 1 \\ 10.0 & 1 \\ 1.0 & 1 \\ 1.0 & 1 \\ 1.0 & 1 \\ 5.0 & 1 \\ 1.0 & 1 \\ 1.0 & 1 \\ 1.0 & 1 \\ 1.0 & 1 \\ 1.0 & 1 \\ 1.0 & 1 \\ 1.0 & 1 \\ 2.5 & 1 \\ 1.0 & 1 \\ 1.0 & 1 \\ 1.0 & 1 \\ 1.0 & 1 \\ 1.0 & 1 \\ 1.0 & 1 \\ 1.0 & 1 \\ 1.0 & 1 \\ 1.0 & 1 \\ 1.0 & 1 \\ 1.0 & 1 \\ 1.0 & 1 \\ 1.0 & 1 \\ 1.0 & 1 \\ 1.0 & 1 \\ 1.0 & 1 \\ 1.0 & 1 \\ 1.0 & 1 \\ 1.0 & 1 \\ 1.0 & 1 \\ 1.0 & 1 \\ 10.0 & 1 \\ 1.0 & 1 \\ 1.0 & 1 \\ & \end{array}$

10/03/10 13:01 67-64-1

10/03/10 13:01 71-43-2

10/03/10 13:01 108-86-1

10/03/10 13:01 74-97-5

10/03/10 13:01 75-27-4

10/03/10 13:01 75-25-2

10/03/10 13:01 74-83-9

10/03/10 13:01 78-93-3

10/03/10 13:01 104-51-8

10/03/10 13:01 135-98-8

10/03/10 13:01 98-06-6

10/03/10 13:01 75-15-0

10/03/10 13:01 56-23-5

10/03/10 13:01 108-90-7

10/03/10 13:01 75-00-3

10/03/10 13:01 67-66-3

10/03/10 13:01 74-87-3

10/03/10 13:01 95-49-8

10/03/10 13:01 106-43-4

10/03/10 13:01 96-12-8

10/03/10 13:01 124-48-1

$10 / 03 / 10$ 13:01 106-93-4

10/03/10 13:01 74-95-3

10/03/10 13:01 95-50-1

10/03/10 13:01 541-73-1

$10 / 03 / 10$ 13:01 106-46-7

10/03/10 13:01 75-71-8

10/03/10 13:01 75-34-3

10/03/10 13:01 107-06-2

10/03/10 13:01 540-59-0

10/03/10 13:01 75-35-4

10/03/10 13:01 156-59-2

10/03/10 13:01 156-60-5

10/03/10 13:01 78-87-5

10/03/10 13:01 142-28-9

10/03/10 13:01 594-20-7

10/03/10 13:01 563-58-6

10/03/10 13:01 10061-01-5

10/03/10 13:01 10061-02-6

10/03/10 13:01 100-41-4

10/03/10 13:01 87-68-3

10/03/10 13:01 591-78-6

10/03/10 13:01 98-82-8

10/03/10 13:01 99-87-6
L3

L3

Page 7 of 19

This report shall not be reproduced, except in full,

without the written consent of Pace Analytical Services, Inc. 


\section{ANALYTICAL RESULTS}

Project: Kansas Waste Water

Pace Project No.: 6086606

Sample: CNPURGE-W-930102 Lab ID: $6086606002 \quad$ Collected: 09/30/10 10:00 Received: 10/01/10 09:15 Matrix: Water

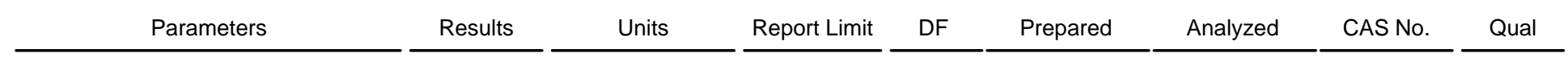

8260 MSV

Methylene chloride

4-Methyl-2-pentanone (MIBK)

Methyl-tert-butyl ether

Naphthalene

n-Propylbenzene

Styrene

1,1,1,2-Tetrachloroethane

1,1,2,2-Tetrachloroethane

Tetrachloroethene

Toluene

1,2,3-Trichlorobenzene

1,2,4-Trichlorobenzene

1,1,1-Trichloroethane

1,1,2-Trichloroethane

Trichloroethene

Trichlorofluoromethane

1,2,3-Trichloropropane

1,2,4-Trimethylbenzene

1,3,5-Trimethylbenzene

Vinyl chloride

Xylene (Total)

4-Bromofluorobenzene (S)

Dibromofluoromethane (S)

1,2-Dichloroethane-d4 (S)

Toluene-d8 (S)

Preservation $\mathrm{pH}$

\subsection{IC Anions}

Nitrate as $\mathrm{N}$
Analytical Method: EPA 5030B/8260

$\begin{array}{lrrll}\text { ND ug/L } & 1.0 & 1 & 10 / 03 / 1013: 01 & 75-09-2 \\ \text { ND ug/L } & 10.0 & 1 & 10 / 03 / 1013: 01 & 108-10-1 \\ \text { ND ug/L } & 1.0 & 1 & 10 / 03 / 1013: 01 & 1634-04-4 \\ \text { ND ug/L } & 10.0 & 1 & 10 / 03 / 1013: 01 & 91-20-3 \\ \text { ND ug/L } & 1.0 & 1 & 10 / 03 / 1013: 01 & 103-65-1 \\ \text { ND ug/L } & 1.0 & 1 & 10 / 03 / 1013: 01 & 100-42-5 \\ \text { ND ug/L } & 1.0 & 1 & 10 / 03 / 1013: 01 & 630-20-6 \\ \text { ND ug/L } & 1.0 & 1 & 10 / 03 / 1013: 01 & 79-34-5 \\ \text { ND ug/L } & 1.0 & 1 & 10 / 03 / 1013: 01 & 127-18-4 \\ \text { ND ug/L } & 1.0 & 1 & 10 / 03 / 1013: 01 & 108-88-3 \\ \text { ND ug/L } & 1.0 & 1 & 10 / 03 / 1013: 01 & 87-61-6 \\ \text { ND ug/L } & 1.0 & 1 & 10 / 03 / 1013: 01 & 120-82-1 \\ \text { ND ug/L } & 1.0 & 1 & 10 / 03 / 1013: 01 & 71-55-6 \\ \text { ND ug/L } & 1.0 & 1 & 10 / 03 / 1013: 01 & 79-00-5 \\ \text { ND ug/L } & 1.0 & 1 & 10 / 03 / 1013: 01 & 79-01-6 \\ \text { ND ug/L } & 1.0 & 1 & 10 / 03 / 1013: 01 & 75-69-4 \\ \text { ND ug/L } & 2.5 & 1 & 10 / 03 / 1013: 01 & 96-18-4 \\ \text { ND ug/L } & 1.0 & 1 & 10 / 03 / 1013: 01 & 95-63-6 \\ \text { ND ug/L } & 1.0 & 1 & 10 / 03 / 1013: 01 & 108-67-8 \\ \text { ND ug/L } & 1.0 & 1 & 10 / 03 / 1013: 01 & 75-01-4 \\ \text { ND ug/L } & 3.0 & 1 & 10 / 03 / 1013: 01 & 1330-20-7 \\ 96 \% & 87-113 & 1 & 10 / 03 / 1013: 01 & 460-00-4 \\ 105 \% & 86-112 & 1 & 10 / 03 / 1013: 01 & 1868-53-7 \\ 100 \% & 82-119 & 1 & 10 / 03 / 1013: 01 & 17060-07-0 \\ 101 \% & 90-110 & 1 & 10 / 03 / 1013: 01 & 2037-26-5 \\ 7.0 & 0.10 & 1 & 10 / 03 / 1013: 01 & \end{array}$

Analytical Method: EPA 300.0

$1.7 \mathrm{mg} / \mathrm{L}$

$0.10 \quad 1$

10/01/10 19:15 14797-55-8

This report shall not be reproduced, except in full, without the written consent of Pace Analytical Services, Inc. 


\section{ANALYTICAL RESULTS}

Project: Kansas Waste Water

Pace Project No.: 6086606

Sample: EVPURGE-W-930103 Lab ID: 6086606003 Collected: 09/30/10 11:32 Received: 10/01/10 09:15 Matrix: Water

\begin{tabular}{|c|c|}
\hline Parameters & Results \\
\hline
\end{tabular}

504 GCS EDB and DBCP

1,2-Dibromoethane (EDB)

\section{MSV}

Acetone

Benzene

Bromobenzene

Bromochloromethane

Bromodichloromethane

Bromoform

Bromomethane

2-Butanone (MEK)

n-Butylbenzene

sec-Butylbenzene

tert-Butylbenzene

Carbon disulfide

Carbon tetrachloride

Chlorobenzene

Chloroethane

Chloroform

Chloromethane

2-Chlorotoluene

4-Chlorotoluene

1,2-Dibromo-3-chloropropane

Dibromochloromethane

1,2-Dibromoethane (EDB)

Dibromomethane

1,2-Dichlorobenzene

1,3-Dichlorobenzene

1,4-Dichlorobenzene

Dichlorodifluoromethane

1,1-Dichloroethane

1,2-Dichloroethane

1,2-Dichloroethene (Total)

1,1-Dichloroethene

cis-1,2-Dichloroethene

trans-1,2-Dichloroethene

1,2-Dichloropropane

1,3-Dichloropropane

2,2-Dichloropropane

1,1-Dichloropropene

cis-1,3-Dichloropropene

trans-1,3-Dichloropropene

Ethylbenzene

Hexachloro-1,3-butadiene

2-Hexanone

Isopropylbenzene (Cumene)

p-Isopropyltoluene

Date: 10/14/2010 11:06 AM
Analytical Method: EPA 504.1 Preparation Method: EPA 504.1

$$
\begin{array}{llllllll}
\text { ND ug/L } & 0.029 & 1 & 10 / 07 / 10 & 00: 00 & 10 / 07 / 10 & 21: 44 & 106-93-4
\end{array}
$$

Analytical Method: EPA 5030B/8260

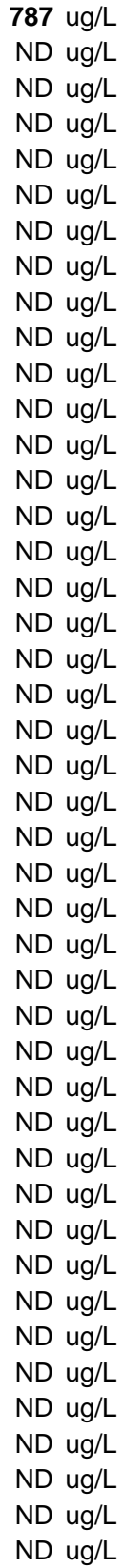

\begin{tabular}{rr}
10.0 & 1 \\
1.0 & 1 \\
1.0 & 1 \\
1.0 & 1 \\
1.0 & 1 \\
1.0 & 1 \\
1.0 & 1 \\
10.0 & 1 \\
1.0 & 1 \\
1.0 & 1 \\
1.0 & 1 \\
5.0 & 1 \\
1.0 & 1 \\
1.0 & 1 \\
1.0 & 1 \\
1.0 & 1 \\
1.0 & 1 \\
1.0 & 1 \\
1.0 & 1 \\
2.5 & 1 \\
1.0 & 1 \\
1.0 & 1 \\
1.0 & 1 \\
1.0 & 1 \\
1.0 & 1 \\
1.0 & 1 \\
1.0 & 1 \\
1.0 & 1 \\
1.0 & 1 \\
1.0 & 1 \\
1.0 & 1 \\
1.0 & 1 \\
1.0 & 1 \\
1.0 & 1 \\
1.0 & 1 \\
1.0 & 1 \\
1.0 & 1 \\
1.0 & 1 \\
1.0 & 1 \\
1.0 & 1 \\
1.0 & 1 \\
10.0 & 1 \\
1.0 & 1 \\
1.0 & 1 \\
& \\
\hline
\end{tabular}

10/03/10 13:16 67-64-1

$10 / 03 / 1013: 16 \quad 71-43-2$

$10 / 03 / 1013: 16 \quad 108-86-1$

10/03/10 13:16 74-97-5

$10 / 03 / 10$ 13:16 75-27-4

$10 / 03 / 10$ 13:16 75-25-2

10/03/10 13:16 74-83-9

10/03/10 13:16 78-93-3

$10 / 03 / 1013: 16 \quad 104-51-8$

10/03/10 13:16 135-98-8

10/03/10 13:16 98-06-6

10/03/10 13:16 75-15-0

$10 / 03 / 1013: 16 \quad 56-23-5$

10/03/10 13:16 108-90-7

10/03/10 13:16 75-00-3

10/03/10 13:16 67-66-3

10/03/10 13:16 74-87-3

10/03/10 13:16 95-49-8

10/03/10 13:16 106-43-4

10/03/10 13:16 96-12-8

10/03/10 13:16 124-48-1

$10 / 03 / 1013: 16 \quad 106-93-4$

10/03/10 13:16 74-95-3

10/03/10 13:16 95-50-1

10/03/10 13:16 541-73-1

10/03/10 13:16 106-46-7

10/03/10 13:16 75-71-8

10/03/10 13:16 75-34-3

$10 / 03 / 1013: 16 \quad 107-06-2$

10/03/10 13:16 540-59-0

10/03/10 13:16 75-35-4

10/03/10 13:16 156-59-2

$10 / 03 / 1013: 16 \quad 156-60-5$

10/03/10 13:16 78-87-5

10/03/10 13:16 142-28-9

10/03/10 13:16 594-20-7

10/03/10 13:16 563-58-6

10/03/10 13:16 10061-01-5

10/03/10 13:16 10061-02-6

$10 / 03 / 1013: 16 \quad 100-41-4$

10/03/10 13:16 87-68-3

10/03/10 13:16 591-78-6

10/03/10 13:16 98-82-8

10/03/10 13:16 99-87-6
E,P2

L3

L3

\section{REPORT OF LABORATORY ANALYSIS}

This report shall not be reproduced, except in full,

without the written consent of Pace Analytical Services, Inc. 


\section{ANALYTICAL RESULTS}

Project: Kansas Waste Water

Pace Project No.: 6086606

Sample: EVPURGE-W-930103 Lab ID: $6086606003 \quad$ Collected: 09/30/10 11:32 Received: 10/01/10 09:15 Matrix: Water

Parameters $\longrightarrow$ Results $\longrightarrow$ Units $\longrightarrow$ Report Limit $\longrightarrow$ DF Prepared $\longrightarrow$ Analyzed CAS No.

8260 MSV

Methylene chloride

4-Methyl-2-pentanone (MIBK)

Methyl-tert-butyl ether

Naphthalene

n-Propylbenzene

Styrene

1,1,1,2-Tetrachloroethane

1,1,2,2-Tetrachloroethane

Tetrachloroethene

Toluene

1,2,3-Trichlorobenzene

1,2,4-Trichlorobenzene

1,1,1-Trichloroethane

1,1,2-Trichloroethane

Trichloroethene

Trichlorofluoromethane

1,2,3-Trichloropropane

1,2,4-Trimethylbenzene

1,3,5-Trimethylbenzene

Vinyl chloride

Xylene (Total)

4-Bromofluorobenzene (S)

Dibromofluoromethane (S)

1,2-Dichloroethane-d4 (S)

Toluene-d8 (S)

Preservation $\mathrm{pH}$

\subsection{IC Anions}

Nitrate as $\mathrm{N}$
Analytical Method: EPA 5030B/8260

\begin{tabular}{|c|c|c|c|c|}
\hline ND ug/L & 1.0 & 1 & $10 / 03 / 1013: 16$ & $75-09-2$ \\
\hline ND ug/L & 10.0 & 1 & $10 / 03 / 1013: 16$ & $108-10-1$ \\
\hline ND ug/L & 1.0 & 1 & $10 / 03 / 1013: 16$ & $1634-04-4$ \\
\hline ND ug/L & 10.0 & 1 & 10/03/10 13:16 & $91-20-3$ \\
\hline ND ug/L & 1.0 & 1 & $10 / 03 / 1013: 16$ & $103-65-1$ \\
\hline ND ug/L & 1.0 & 1 & $10 / 03 / 1013: 16$ & $100-42-5$ \\
\hline ND ug/L & 1.0 & 1 & 10/03/10 13:16 & $630-20-6$ \\
\hline ND ug/L & 1.0 & 1 & $10 / 03 / 1013: 16$ & $79-34-5$ \\
\hline ND ug/L & 1.0 & 1 & $10 / 03 / 1013: 16$ & $127-18-4$ \\
\hline ND ug/L & 1.0 & 1 & 10/03/10 13:16 & $108-88-3$ \\
\hline ND ug/L & 1.0 & 1 & $10 / 03 / 1013: 16$ & $87-61-6$ \\
\hline ND ug/L & 1.0 & 1 & $10 / 03 / 1013: 16$ & $120-82-1$ \\
\hline ND ug/L & 1.0 & 1 & 10/03/10 13:16 & $71-55-6$ \\
\hline ND ug/L & 1.0 & 1 & $10 / 03 / 1013: 16$ & $79-00-5$ \\
\hline ND ug/L & 1.0 & 1 & $10 / 03 / 1013: 16$ & $79-01-6$ \\
\hline ND ug/L & 1.0 & 1 & 10/03/10 13:16 & $75-69-4$ \\
\hline ND ug/L & 2.5 & 1 & $10 / 03 / 1013: 16$ & $96-18-4$ \\
\hline ND ug/L & 1.0 & 1 & $10 / 03 / 1013: 16$ & $95-63-6$ \\
\hline ND ug/L & 1.0 & 1 & $10 / 03 / 1013: 16$ & $108-67-8$ \\
\hline ND ug/L & 1.0 & 1 & $10 / 03 / 1013: 16$ & $75-01-4$ \\
\hline ND ug/L & 3.0 & 1 & 10/03/10 13:16 & $1330-20-7$ \\
\hline $96 \%$ & $87-113$ & 1 & 10/03/10 13:16 & $460-00-4$ \\
\hline $98 \%$ & $86-112$ & 1 & $10 / 03 / 1013: 16$ & $1868-53-7$ \\
\hline $92 \%$ & $82-119$ & 1 & $10 / 03 / 1013: 16$ & $17060-07-0$ \\
\hline $100 \%$ & $90-110$ & 1 & 10/03/10 13:16 & $2037-26-5$ \\
\hline 7.0 & 0.10 & 1 & $10 / 03 / 1013: 16$ & \\
\hline
\end{tabular}

Analytical Method: EPA 300.0

$2.0 \mathrm{mg} / \mathrm{L}$

$0.10 \quad 1$

10/01/10 19:32 14797-55-8

This report shall not be reproduced, except in full, without the written consent of Pace Analytical Services, Inc. 


\section{ANALYTICAL RESULTS}

Project: Kansas Waste Water

Pace Project No.: 6086606

Sample: MRPURGE-W-930104 Lab ID: 6086606004 Collected: 09/30/10 13:42 Received: 10/01/10 09:15 Matrix: Water

\begin{tabular}{|c|c|}
\hline Parameters & Results \\
\hline
\end{tabular}

504 GCS EDB and DBCP

1,2-Dibromoethane (EDB)

\section{MSV}

Acetone

Benzene

Bromobenzene

Bromochloromethane

Bromodichloromethane

Bromoform

Bromomethane

2-Butanone (MEK)

n-Butylbenzene

sec-Butylbenzene

tert-Butylbenzene

Carbon disulfide

Carbon tetrachloride

Chlorobenzene

Chloroethane

Chloroform

Chloromethane

2-Chlorotoluene

4-Chlorotoluene

1,2-Dibromo-3-chloropropane

Dibromochloromethane

1,2-Dibromoethane (EDB)

Dibromomethane

1,2-Dichlorobenzene

1,3-Dichlorobenzene

1,4-Dichlorobenzene

Dichlorodifluoromethane

1,1-Dichloroethane

1,2-Dichloroethane

1,2-Dichloroethene (Total)

1,1-Dichloroethene

cis-1,2-Dichloroethene

trans-1,2-Dichloroethene

1,2-Dichloropropane

1,3-Dichloropropane

2,2-Dichloropropane

1,1-Dichloropropene

cis-1,3-Dichloropropene

trans-1,3-Dichloropropene

Ethylbenzene

Hexachloro-1,3-butadiene

2-Hexanone

Isopropylbenzene (Cumene)

p-Isopropyltoluene

Date: 10/14/2010 11:06 AM

Analytical Method: EPA 504.1 Preparation Method: EPA 504.1

$$
\begin{array}{llllllll}
\text { ND ug/L } & 0.029 & 1 & 10 / 07 / 10 & 00: 00 & 10 / 07 / 10 & 21: 55 & 106-93-4
\end{array}
$$

Analytical Method: EPA 5030B/8260

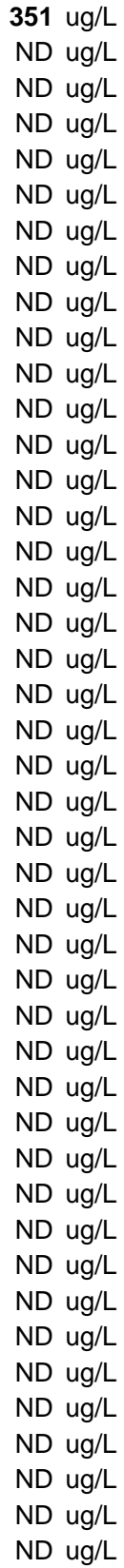

$10.0 \quad 1$

$1.0 \quad 1$

$1.0 \quad 1$

$1.0 \quad 1$

$1.0 \quad 1$

1.01

1.01

10.01

$1.0 \quad 1$

$1.0 \quad 1$

$1.0 \quad 1$

5.01

$1.0 \quad 1$

$1.0 \quad 1$

$1.0 \quad 1$

$1.0 \quad 1$

$1.0 \quad 1$

$1.0 \quad 1$

$1.0 \quad 1$

2.51

$1.0 \quad 1$

$1.0 \quad 1$

$1.0 \quad 1$

$1.0 \quad 1$

$1.0 \quad 1$

1.01

$1.0 \quad 1$

$1.0 \quad 1$

1.01

$1.0 \quad 1$

$1.0 \quad 1$

$1.0 \quad 1$

$1.0 \quad 1$

$1.0 \quad 1$

$1.0 \quad 1$

$1.0 \quad 1$

$1.0 \quad 1$

$1.0 \quad 1$

$1.0 \quad 1$

1.01

1.01

$10.0 \quad 1$

$1.0 \quad 1$

1.01
10/03/10 13:31 67-64-1

10/03/10 13:31 71-43-2

10/03/10 13:31 108-86-1

10/03/10 13:31 74-97-5

10/03/10 13:31 75-27-4

10/03/10 13:31 75-25-2

10/03/10 13:31 74-83-9

10/03/10 13:31 78-93-3

10/03/10 13:31 104-51-8

$10 / 03 / 1013: 31 \quad 135-98-8$

10/03/10 13:31 98-06-6

10/03/10 13:31 75-15-0

10/03/10 13:31 56-23-5

10/03/10 13:31 108-90-7

10/03/10 13:31 75-00-3

10/03/10 13:31 67-66-3

10/03/10 13:31 74-87-3

10/03/10 13:31 95-49-8

$10 / 03 / 10$ 13:31 106-43-4

10/03/10 13:31 96-12-8

10/03/10 13:31 124-48-1

10/03/10 13:31 106-93-4

10/03/10 13:31 74-95-3

10/03/10 13:31 95-50-1

10/03/10 13:31 541-73-1

10/03/10 13:31 106-46-7

10/03/10 13:31 75-71-8

10/03/10 13:31 75-34-3

10/03/10 13:31 107-06-2

10/03/10 13:31 540-59-0

10/03/10 13:31 75-35-4

10/03/10 13:31 156-59-2

10/03/10 13:31 156-60-5

10/03/10 13:31 78-87-5

10/03/10 13:31 142-28-9

$10 / 03 / 1013: 31 \quad 594-20-7$

10/03/10 13:31 563-58-6

10/03/10 13:31 10061-01-5

10/03/10 13:31 10061-02-6

$10 / 03 / 1013: 31 \quad 100-41-4$

10/03/10 13:31 87-68-3

10/03/10 13:31 591-78-6

$10 / 03 / 1013: 31 \quad 98-82-8$

$10 / 03 / 1013: 31 \quad 99-87-6$
L3

L3

L3

Page 11 of 19

\section{REPORT OF LABORATORY ANALYSIS}

This report shall not be reproduced, except in full,

without the written consent of Pace Analytical Services, Inc.
3 


\section{ANALYTICAL RESULTS}

Project: Kansas Waste Water

Pace Project No.: 6086606

Sample: MRPURGE-W-930104 Lab ID: 6086606004 Collected: 09/30/10 13:42 $\quad$ Received: 10/01/10 09:15 Matrix: Water

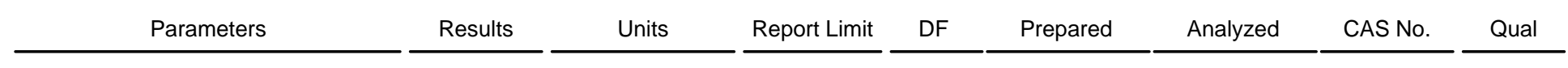

8260 MSV

Methylene chloride

4-Methyl-2-pentanone (MIBK)

Methyl-tert-butyl ether

Naphthalene

n-Propylbenzene

Styrene

1,1,1,2-Tetrachloroethane

1,1,2,2-Tetrachloroethane

Tetrachloroethene

Toluene

1,2,3-Trichlorobenzene

1,2,4-Trichlorobenzene

1,1,1-Trichloroethane

1,1,2-Trichloroethane

Trichloroethene

Trichlorofluoromethane

1,2,3-Trichloropropane

1,2,4-Trimethylbenzene

1,3,5-Trimethylbenzene

Vinyl chloride

Xylene (Total)

4-Bromofluorobenzene (S)

Dibromofluoromethane (S)

1,2-Dichloroethane-d4 (S)

Toluene-d8 (S)

Preservation $\mathrm{pH}$

\subsection{IC Anions}

Nitrate as $\mathrm{N}$

Analytical Method: EPA 5030B/8260

\begin{abstract}
ND ug/L
ND ug/L

ND ug/L

ND ug/L

ND ug/L

ND ug/L

ND ug/L

ND ug/L

ND ug/L

ND ug/L

ND ug/L

ND ug/L

ND ug/L

ND ug/L

ND ug/L

ND ug/L

ND ug/L

ND ug/L

ND ug/L

ND ug/L

ND ug/L

$95 \%$

$105 \%$

$98 \%$

$103 \%$
\end{abstract}

7.0

Analytical Method: EPA 300.0

$0.99 \mathrm{mg} / \mathrm{L}$

$\begin{array}{rr}1.0 & 1 \\ 10.0 & 1 \\ 1.0 & 1 \\ 10.0 & 1 \\ 1.0 & 1 \\ 1.0 & 1 \\ 1.0 & 1 \\ 1.0 & 1 \\ 1.0 & 1 \\ 1.0 & 1 \\ 1.0 & 1 \\ 1.0 & 1 \\ 1.0 & 1 \\ 1.0 & 1 \\ 1.0 & 1 \\ 1.0 & 1 \\ 2.5 & 1 \\ 1.0 & 1 \\ 1.0 & 1 \\ 1.0 & 1 \\ 3.0 & 1 \\ 87-113 & 1 \\ 86-112 & 1 \\ 82-119 & 1 \\ 90-110 & 1 \\ 0.10 & 1\end{array}$

$0.10 \quad 1$
10/03/10 13:31 75-09-2

10/03/10 13:31 108-10-1

10/03/10 13:31 1634-04-4

10/03/10 13:31 91-20-3

10/03/10 13:31 103-65-1

10/03/10 13:31 100-42-5

10/03/10 13:31 630-20-6

10/03/10 13:31 79-34-5

10/03/10 13:31 127-18-4

$10 / 03 / 10$ 13:31 108-88-3

10/03/10 13:31 87-61-6

$10 / 03 / 1013: 31 \quad 120-82-1$

10/03/10 13:31 71-55-6

10/03/10 13:31 79-00-5

10/03/10 13:31 79-01-6

10/03/10 13:31 75-69-4

10/03/10 13:31 96-18-4

10/03/10 13:31 95-63-6

10/03/10 13:31 108-67-8

10/03/10 13:31 75-01-4

10/03/10 13:31 1330-20-7

10/03/10 13:31 460-00-4

10/03/10 13:31 1868-53-7

10/03/10 13:31 17060-07-0

10/03/10 13:31 2037-26-5

10/03/10 13:31

10/01/10 19:48 14797-55-8

This report shall not be reproduced, except in full, without the written consent of Pace Analytical Services, Inc. 


\section{QUALITY CONTROL DATA}

Project: Kansas Waste Water

Pace Project No.: 6086606

\begin{tabular}{llll}
\hline QC Batch: & OEXT/25923 & Analysis Method: & EPA 504.1 \\
QC Batch Method: & EPA 504.1 & Analysis Description: & GCS 504 EDB DBCP
\end{tabular}

Associated Lab Samples: $\quad$ 6086606001, 6086606002, 6086606003, 6086606004

METHOD BLANK: $713551 \quad$ Matrix: Water

Associated Lab Samples: $\quad$ 6086606001, 6086606002, 6086606003, 6086606004

$\frac{\text { Parameter }}{1,2 \text {-Dibromoethane (EDB) }} \frac{\text { Units }}{\mathrm{ug} / \mathrm{L}} \frac{\text { Result }}{\mathrm{ND}} \frac{\begin{array}{c}\text { Blank } \\ \text { Limit }\end{array}}{0.030} \frac{\text { Analyzed }}{10 / 07 / 1020: 50} \stackrel{\text { Qualifiers }}{-}$

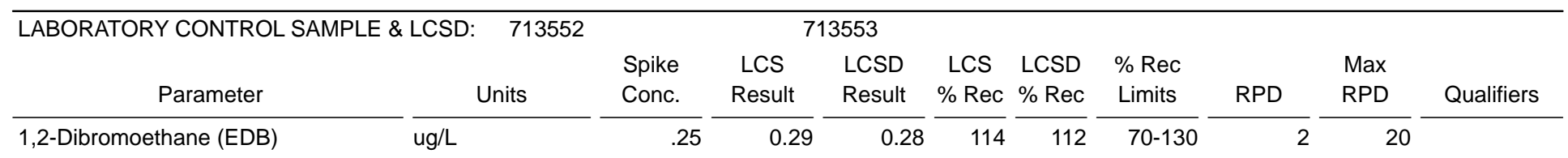




\section{QUALITY CONTROL DATA}

Project: Kansas Waste Water

Pace Project No.: 6086606

\begin{tabular}{llll}
\hline QC Batch: & MSV/32160 & Analysis Method: & EPA 5030B/8260 \\
QC Batch Method: & EPA 5030B/8260 & Analysis Description: & 8260 MSV Water 7 day
\end{tabular}

Associated Lab Samples: $\quad 6086606001,6086606002,6086606003,6086606004$

\begin{tabular}{|c|c|c|c|c|c|}
\hline \multirow{2}{*}{$\begin{array}{l}\text { METHOD BLANK: } 711291 \\
\text { Associated Lab Samples: }\end{array}$} & \multicolumn{4}{|c|}{ Matrix: Water } & \multirow[b]{3}{*}{ Qualifiers } \\
\hline & 001,6086606 & 506003,6086 & 06004 & & \\
\hline Parameter & Units & $\begin{array}{l}\text { Blank } \\
\text { Result }\end{array}$ & $\begin{array}{l}\text { Reporting } \\
\text { Limit }\end{array}$ & Analyzed & \\
\hline 1,1,1,2-Tetrachloroethane & $u g / L$ & ND & 1.0 & $10 / 03 / 1011: 44$ & \\
\hline 1,1,1-Trichloroethane & $\mathrm{ug} / \mathrm{L}$ & ND & 1.0 & $10 / 03 / 1011: 44$ & \\
\hline 1,1,2,2-Tetrachloroethane & $u g / L$ & ND & 1.0 & $10 / 03 / 1011: 44$ & \\
\hline 1,1,2-Trichloroethane & $u g / L$ & ND & 1.0 & $10 / 03 / 1011: 44$ & \\
\hline 1,1-Dichloroethane & $u g / L$ & ND & 1.0 & $10 / 03 / 1011: 44$ & \\
\hline 1,1-Dichloroethene & $\mathrm{ug} / \mathrm{L}$ & ND & 1.0 & $10 / 03 / 1011: 44$ & \\
\hline 1,1-Dichloropropene & $u g / L$ & ND & 1.0 & $10 / 03 / 1011: 44$ & \\
\hline 1,2,3-Trichlorobenzene & $\mathrm{ug} / \mathrm{L}$ & ND & 1.0 & $10 / 03 / 1011: 44$ & \\
\hline 1,2,3-Trichloropropane & $\mathrm{ug} / \mathrm{L}$ & ND & 2.5 & $10 / 03 / 1011: 44$ & \\
\hline 1,2,4-Trichlorobenzene & $u g / L$ & ND & 1.0 & $10 / 03 / 1011: 44$ & \\
\hline 1,2,4-Trimethylbenzene & $\mathrm{ug} / \mathrm{L}$ & ND & 1.0 & $10 / 03 / 1011: 44$ & \\
\hline 1,2-Dibromo-3-chloropropane & $\mathrm{ug} / \mathrm{L}$ & ND & 2.5 & $10 / 03 / 1011: 44$ & \\
\hline 1,2-Dibromoethane (EDB) & ug/L & ND & 1.0 & $10 / 03 / 1011: 44$ & \\
\hline 1,2-Dichlorobenzene & $u g / L$ & ND & 1.0 & $10 / 03 / 1011: 44$ & \\
\hline 1,2-Dichloroethane & $\mathrm{ug} / \mathrm{L}$ & ND & 1.0 & $10 / 03 / 1011: 44$ & \\
\hline 1,2-Dichloroethene (Total) & $\mathrm{ug} / \mathrm{L}$ & ND & 1.0 & $10 / 03 / 1011: 44$ & \\
\hline 1,2-Dichloropropane & $u g / L$ & ND & 1.0 & $10 / 03 / 1011: 44$ & \\
\hline 1,3,5-Trimethylbenzene & $u g / L$ & ND & 1.0 & $10 / 03 / 1011: 44$ & \\
\hline 1,3-Dichlorobenzene & $\mathrm{ug} / \mathrm{L}$ & ND & 1.0 & $10 / 03 / 1011: 44$ & \\
\hline 1,3-Dichloropropane & $u g / L$ & ND & 1.0 & $10 / 03 / 1011: 44$ & \\
\hline 1,4-Dichlorobenzene & ug/L & ND & 1.0 & $10 / 03 / 1011: 44$ & \\
\hline 2,2-Dichloropropane & $\mathrm{ug} / \mathrm{L}$ & ND & 1.0 & $10 / 03 / 1011: 44$ & \\
\hline 2-Butanone (MEK) & $u g / L$ & ND & 10.0 & $10 / 03 / 1011: 44$ & \\
\hline 2-Chlorotoluene & $\mathrm{ug} / \mathrm{L}$ & ND & 1.0 & $10 / 03 / 1011: 44$ & \\
\hline 2-Hexanone & $\mathrm{ug} / \mathrm{L}$ & ND & 10.0 & $10 / 03 / 1011: 44$ & \\
\hline 4-Chlorotoluene & $u g / L$ & ND & 1.0 & $10 / 03 / 1011: 44$ & \\
\hline 4-Methyl-2-pentanone (MIBK) & $\mathrm{ug} / \mathrm{L}$ & ND & 10.0 & $10 / 03 / 1011: 44$ & \\
\hline Acetone & $\mathrm{ug} / \mathrm{L}$ & ND & 10.0 & $10 / 03 / 1011: 44$ & \\
\hline Benzene & ug/L & ND & 1.0 & $10 / 03 / 1011: 44$ & \\
\hline Bromobenzene & $u g / L$ & ND & 1.0 & $10 / 03 / 1011: 44$ & \\
\hline Bromochloromethane & $\mathrm{ug} / \mathrm{L}$ & ND & 1.0 & $10 / 03 / 1011: 44$ & \\
\hline Bromodichloromethane & $\mathrm{ug} / \mathrm{L}$ & ND & 1.0 & $10 / 03 / 1011: 44$ & \\
\hline Bromoform & $u g / L$ & ND & 1.0 & $10 / 03 / 1011: 44$ & \\
\hline Bromomethane & $\mathrm{ug} / \mathrm{L}$ & ND & 1.0 & $10 / 03 / 1011: 44$ & \\
\hline Carbon disulfide & $\mathrm{ug} / \mathrm{L}$ & ND & 5.0 & $10 / 03 / 1011: 44$ & \\
\hline Carbon tetrachloride & $u g / L$ & ND & 1.0 & $10 / 03 / 1011: 44$ & \\
\hline Chlorobenzene & $\mathrm{ug} / \mathrm{L}$ & ND & 1.0 & $10 / 03 / 1011: 44$ & \\
\hline Chloroethane & $\mathrm{ug} / \mathrm{L}$ & ND & 1.0 & $10 / 03 / 1011: 44$ & \\
\hline Chloroform & $u g / L$ & ND & 1.0 & $10 / 03 / 1011: 44$ & \\
\hline Chloromethane & $\mathrm{ug} / \mathrm{L}$ & ND & 1.0 & $10 / 03 / 1011: 44$ & \\
\hline cis-1,2-Dichloroethene & $\mathrm{ug} / \mathrm{L}$ & ND & 1.0 & $10 / 03 / 1011: 44$ & \\
\hline cis-1,3-Dichloropropene & $u g / L$ & ND & 1.0 & $10 / 03 / 1011: 44$ & \\
\hline Dibromochloromethane & $\mathrm{ug} / \mathrm{L}$ & ND & 1.0 & $10 / 03 / 1011: 44$ & \\
\hline
\end{tabular}

Date: 10/14/2010 11:06 AM

REPORT OF LABORATORY ANALYSIS

Page 14 of 19
This report shall not be reproduced, except in full, without the written consent of Pace Analytical Services, Inc.. 


\section{QUALITY CONTROL DATA}

Project: Kansas Waste Water

Pace Project No.: 6086606

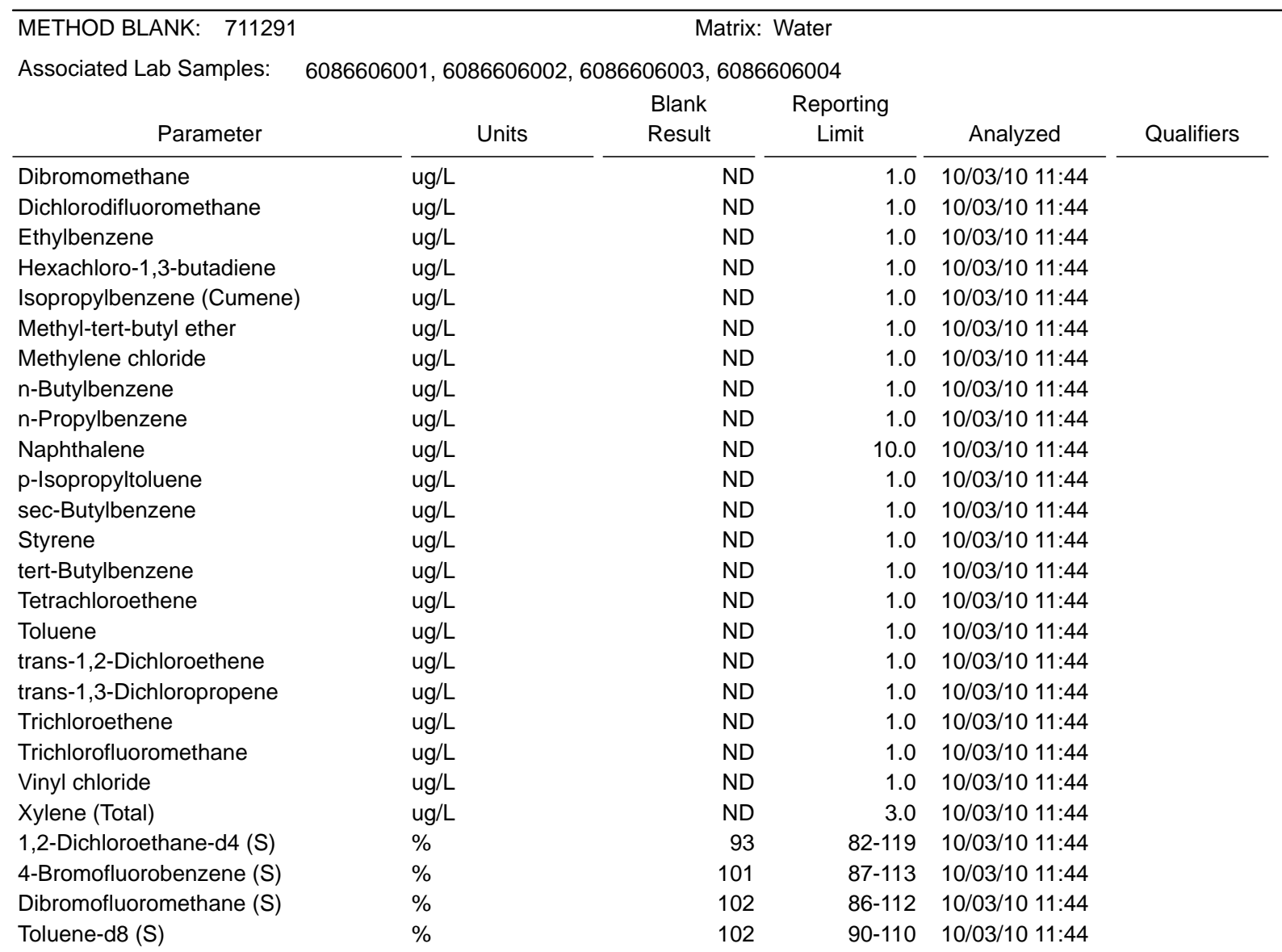

\begin{tabular}{|c|c|c|c|c|c|c|}
\hline \multirow{2}{*}{$\begin{array}{c}\text { LABORATORY CONTROL SAMPLE: } \\
\text { Parameter }\end{array}$} & \multicolumn{6}{|l|}{711292} \\
\hline & Units & $\begin{array}{l}\text { Spike } \\
\text { Conc. }\end{array}$ & $\begin{array}{l}\text { LCS } \\
\text { Result }\end{array}$ & $\begin{array}{c}\text { LCS } \\
\% \operatorname{Rec}\end{array}$ & $\begin{array}{l}\% \text { Rec } \\
\text { Limits }\end{array}$ & Qualifiers \\
\hline $1,1,1,2$-Tetrachloroethane & $u g / L$ & 20 & 22.0 & 110 & $79-116$ & \\
\hline 1,1,1-Trichloroethane & $\mathrm{ug} / \mathrm{L}$ & 20 & 21.2 & 106 & $77-113$ & \\
\hline 1,1,2,2-Tetrachloroethane & $u g / L$ & 20 & 18.9 & 94 & $68-122$ & \\
\hline 1,1,2-Trichloroethane & $u g / L$ & 20 & 20.9 & 104 & $82-117$ & \\
\hline 1,1-Dichloroethane & $u g / L$ & 20 & 20.7 & 103 & $67-122$ & \\
\hline 1,1-Dichloroethene & $u g / L$ & 20 & 23.7 & 118 & $70-119$ & \\
\hline 1,1-Dichloropropene & $u g / L$ & 20 & 21.1 & 106 & $81-115$ & \\
\hline 1,2,3-Trichlorobenzene & ug/L & 20 & 19.9 & 99 & $66-135$ & \\
\hline 1,2,3-Trichloropropane & $u g / L$ & 20 & 18.4 & 92 & $76-126$ & \\
\hline 1,2,4-Trichlorobenzene & $u g / L$ & 20 & 19.7 & 99 & $66-126$ & \\
\hline 1,2,4-Trimethylbenzene & ug/L & 20 & 19.8 & 99 & $78-115$ & \\
\hline 1,2-Dibromo-3-chloropropane & $u g / L$ & 20 & 24.1 & 121 & $58-147$ & \\
\hline 1,2-Dibromoethane (EDB) & $u g / L$ & 20 & 21.2 & 106 & $84-121$ & \\
\hline 1,2-Dichlorobenzene & ug/L & 20 & 20.9 & 105 & $79-116$ & \\
\hline 1,2-Dichloroethane & ug/L & 20 & 19.2 & 96 & 74-119 & \\
\hline
\end{tabular}




\section{QUALITY CONTROL DATA}

Project: Kansas Waste Water

Pace Project No.: 6086606

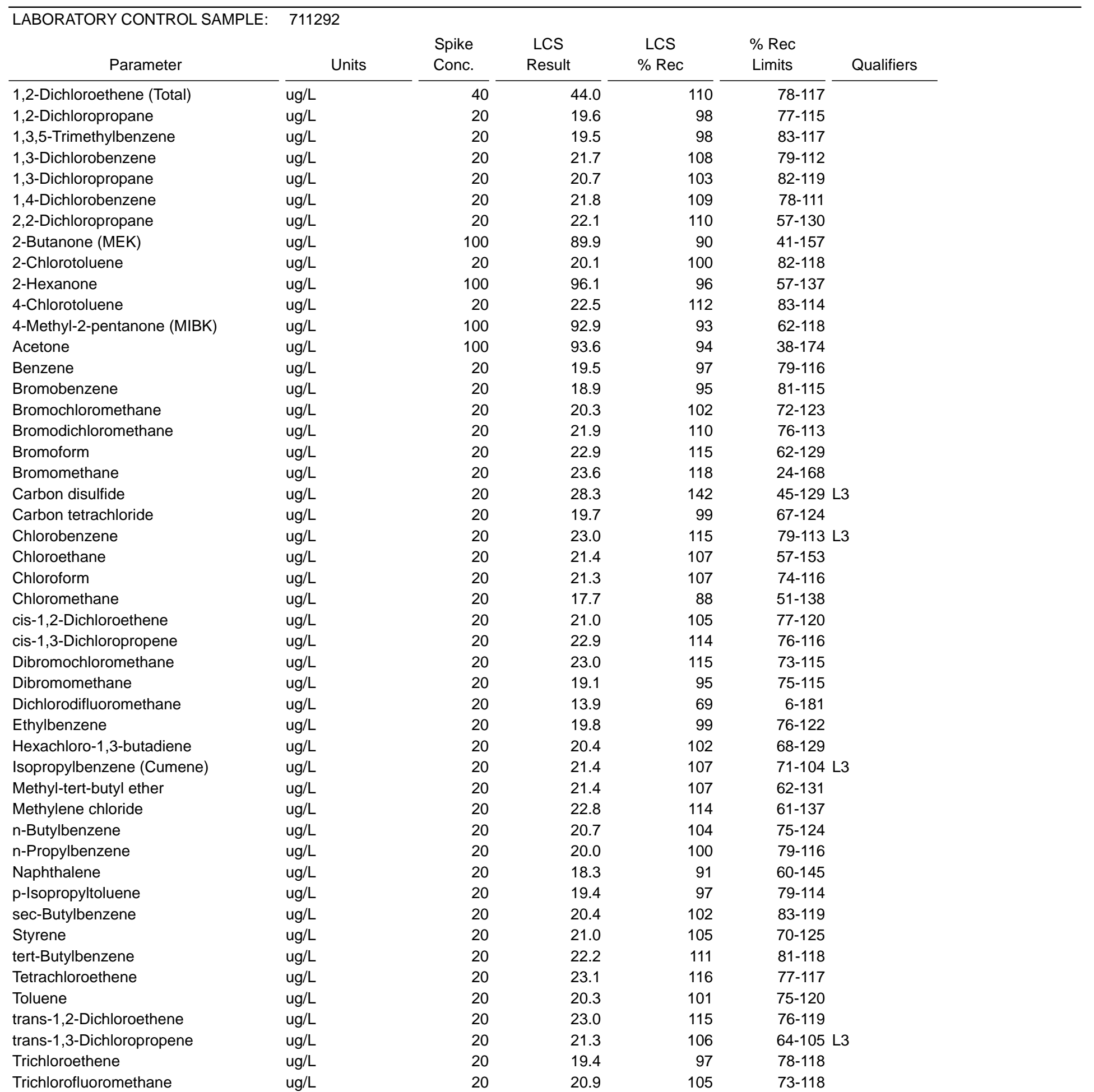

This report shall not be reproduced, except in full, without the written consent of Pace Analytical Services, Inc. 


\section{QUALITY CONTROL DATA}

Project: Kansas Waste Water

Pace Project No.: 6086606

\begin{tabular}{|c|c|c|c|c|c|c|}
\hline LABORATORY CONTROL SAMPLE: & 711292 & & & & & \\
\hline Parameter & Units & $\begin{array}{l}\text { Spike } \\
\text { Conc. }\end{array}$ & $\begin{array}{l}\text { LCS } \\
\text { Result }\end{array}$ & $\begin{array}{l}\text { LCS } \\
\% \operatorname{Rec}\end{array}$ & $\begin{array}{l}\% \operatorname{Rec} \\
\text { Limits }\end{array}$ & Qualifiers \\
\hline Vinyl chloride & $\mathrm{ug} / \mathrm{L}$ & 20 & 22.6 & 113 & $60-122$ & \\
\hline Xylene (Total) & $u g / L$ & 60 & 61.1 & 102 & $74-124$ & \\
\hline 1,2-Dichloroethane-d4 (S) & $\%$ & & & 89 & $82-119$ & \\
\hline 4-Bromofluorobenzene (S) & $\%$ & & & 97 & $87-113$ & \\
\hline Dibromofluoromethane (S) & $\%$ & & & 105 & $86-112$ & \\
\hline Toluene-d8 (S) & $\%$ & & & 101 & $90-110$ & \\
\hline
\end{tabular}

This report shall not be reproduced, except in full, without the written consent of Pace Analytical Services, Inc.. 


\section{QUALITY CONTROL DATA}

Project: Kansas Waste Water

Pace Project No.: 6086606

\begin{tabular}{llll}
\hline QC Batch: & WETA/14191 & Analysis Method: & EPA 300.0 \\
QC Batch Method: & EPA 300.0 & Analysis Description: & 300.0 IC Anions
\end{tabular}

Associated Lab Samples: $\quad 6086606001,6086606002,6086606003,6086606004$

METHOD BLANK: $710224 \quad$ Matrix: Water

Associated Lab Samples: $\quad 6086606001,6086606002,6086606003,6086606004$

$\frac{\text { Parameter }}{\text { Nitrate as } \mathrm{N}} \frac{\text { Units }}{\mathrm{mg} / \mathrm{L}} \frac{\text { Result }}{\mathrm{ND}} \frac{\begin{array}{c}\text { Blank } \\ \text { Limit }\end{array}}{0.10} \frac{\text { Analyzed }}{10 / 01 / 1018: 26} \frac{\text { Qualifiers }}{-}$

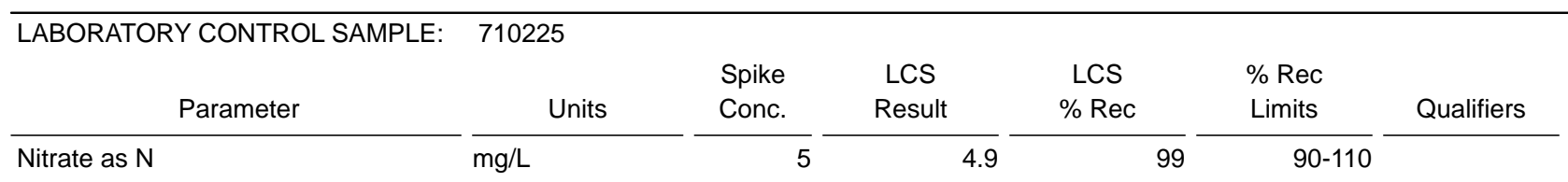

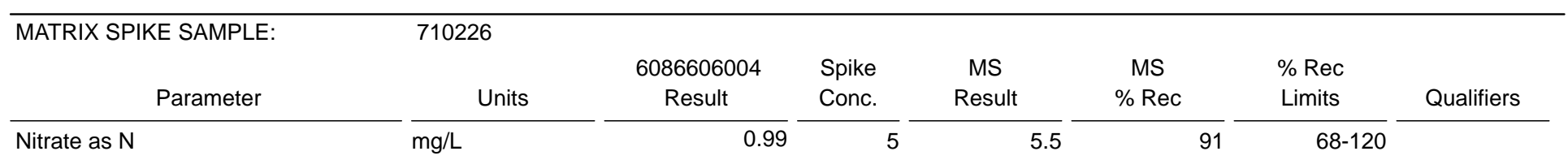

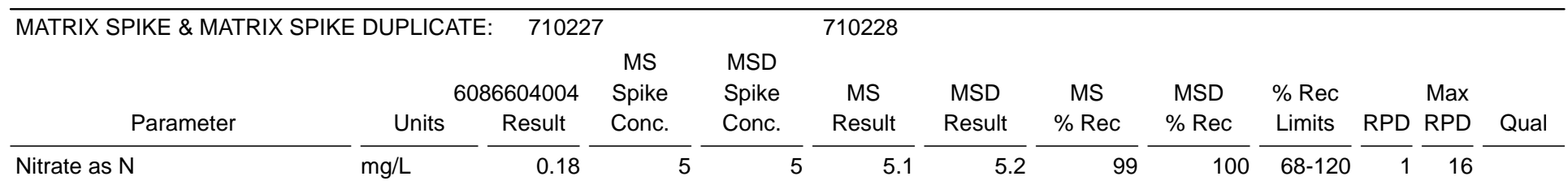




\section{QUALIFIERS}

Project: Kansas Waste Water

Pace Project No.: 6086606

\section{DEFINITIONS}

DF - Dilution Factor, if reported, represents the factor applied to the reported data due to changes in sample preparation, dilution of the sample aliquot, or moisture content.

ND - Not Detected at or above adjusted reporting limit.

$\mathrm{J}$ - Estimated concentration above the adjusted method detection limit and below the adjusted reporting limit.

MDL - Adjusted Method Detection Limit.

$S$ - Surrogate

1,2-Diphenylhydrazine (8270 listed analyte) decomposes to Azobenzene.

Consistent with EPA guidelines, unrounded data are displayed and have been used to calculate \% recovery and RPD values.

LCS(D) - Laboratory Control Sample (Duplicate)

MS(D) - Matrix Spike (Duplicate)

DUP - Sample Duplicate

RPD - Relative Percent Difference

$\mathrm{U}$ - Indicates the compound was analyzed for, but not detected.

N-Nitrosodiphenylamine decomposes and cannot be separated from Diphenylamine using Method 8270. The result reported for each analyte is a combined concentration.

Pace Analytical is NELAP accredited. Contact your Pace PM for the current list of accredited analytes.

\section{BATCH QUALIFIERS}

Batch: MSV/32160

[M5] A matrix spike/matrix spike duplicate was not performed for this batch due to insufficient sample volume.

\section{ANALYTE QUALIFIERS}

E Analyte concentration exceeded the calibration range. The reported result is estimated.

L3 Analyte recovery in the laboratory control sample (LCS) exceeded QC limits. Analyte presence below reporting limits in associated samples. Results unaffected by high bias.

P2 Re-extraction or re-analysis could not be performed due to insufficient sample amount. 


\section{Sample Condition Upon Receipt}

D Pace Analytical
Client Name: TCW
Project \# Co 86606 courier: $\triangle$ Feg Ex $\square$ Uups $\square$ USPS $\square$ client $\square$ Commercial $\square$ pace $\square$ other Tracking \#: 871795231713

Custody Seal on Cooler/Box Present:

Packing Material: $\square$ Bubble Wrapap Thermometer Used:

Cooler Temperature: 2,2

Temperature should be above freezing to $6^{\circ} \mathrm{C}$

Chain of Custody present

Chain of Custody filled out:

Chain of Custody relinquished:

Sampler name \& signature on COC:

Samples arrived within holding time:

Short Hold Time analyses ( $<72 \mathrm{hr})$ :

Rush Turn Around Time requested:

Sufficient volume:

Correct containers used:

-Pace containers used:

Containers intact:

Unpreserved $5035 \mathrm{~A}$ soils frozen w/in $48 \mathrm{hrs}$ ?

Filtered volume received for dissolved tests

Sample labels match COC:

-Includes date/time/ID/analyses Matrix

All containers needing preservation have been checked.

All containers needing preservation are found to be in compliance with EPA recommendation.

Exceptions VOA Coliform, TOC, O\&G, WR-DRO (water),

Phenolics

Trip Blank present:

Pace Trip Blank lot \# (if purchased):

Headspace in VOA vials $(>6 \mathrm{~mm})$ :

Project sampled in USDA Regulated Area:

Client Notification/ Resolution:

Person Contacted:

Pace Shipping Label Used?

$\triangle$ Yes Nolv seals intact: $\square$ Yes

$\triangle$ Yes

$$
\text { bolilo }
$$
Seals in $\square$ Bubble Bags $\square$ Foam Done $\square$ ther

Comments:

DYes $\square$ No $\square$ N/A 5 .

No

No

No

Optional
Proj. Due Date:
Proj. Name:

Type of Ice: WeP Blue None $\square$ Samples on ice, cooling process has begun

Date and Initipłs of person examining contents: Io 17 te by

Gyes $\square$ No $\square$ N/A 1.

Dyes $\square$ No $\square \mathrm{N} / \mathrm{A} 2$.

Dyps $\square$ No $\square \mathrm{N} / \mathrm{A} 3$.

UYes $\square$ No $\square$ N/A 4.

DYes $\square$ No $\square$ N/A 6 . NOJ3

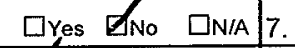

Zyes $\square$ No $\square$ N/A 8

Lres $\square$ No $\square$ N/A 9.

पYes D'No $\square \mathrm{N} / \mathrm{A}$

$\not$ Yres $\square$ No $\square$ N/A 10

$\square$ Yes $\square$ No $\swarrow_{\mathrm{N} / \mathrm{A}} 11$

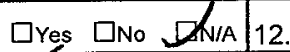

DYes $\square$ No $\square$ N/A 13.

WT

पYes $\square_{\text {No }} Z_{N / \mathrm{A}} 14$.

ares $\square_{\text {No }}$ Dhia

DYes DNg initial when Lot \# of added

\begin{tabular}{l|l}
$\square$ Yes $\square$ Ng & completed $\_$preservative \\
$\square$ Yes $\not$ NNo $\square$ N/A & 15.
\end{tabular}

ares Dtwo aN/A 16.

$\square$ Yes $\square$ No D/N/A 17 17. List State:

Copy COC to Client?

$Y,(N)$

Field Data Required?

$Y / N$

Date/Time:

Comments/Resolution:

Project Manager Review:

Date: $10-1-10$

Note: Whenever there is a discrepancy affecting North Carolina compliance samples, a copy of this form will be sent to the North Carolina DEHNR Certification Office ( i.e out of hold, incorrect preservative, out of temp, incorrect containers) 
Section A

Required Client Information:

Company: TCW Construation

Address: $141 \mathrm{~m}$ Street Lincoln NE 68508

Email To: Phone: 4167255 Fax:

Requested Due Date/TAT:
Section C

Section B

Required Project Information:

Invoice Information:

Report To: Kamlen Q taw construction,com Attention: Travis Kandem Copy To:sargnier@prodigunet

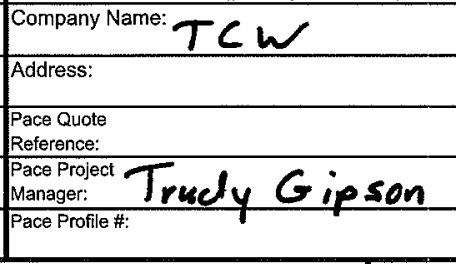

\section{Project Name: Kansas Waste Water}

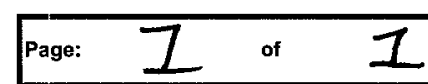

1272142 REGULATORY AGENCY

$\Gamma$ NPDES $\varnothing$ GROUND WATER $\Gamma$ DRINKING WATER

Purchase Order No:

$\Gamma$ OTHER

(2.

$\Gamma$ ust

Site Location

Requested Analysis Filtered (Y/N)

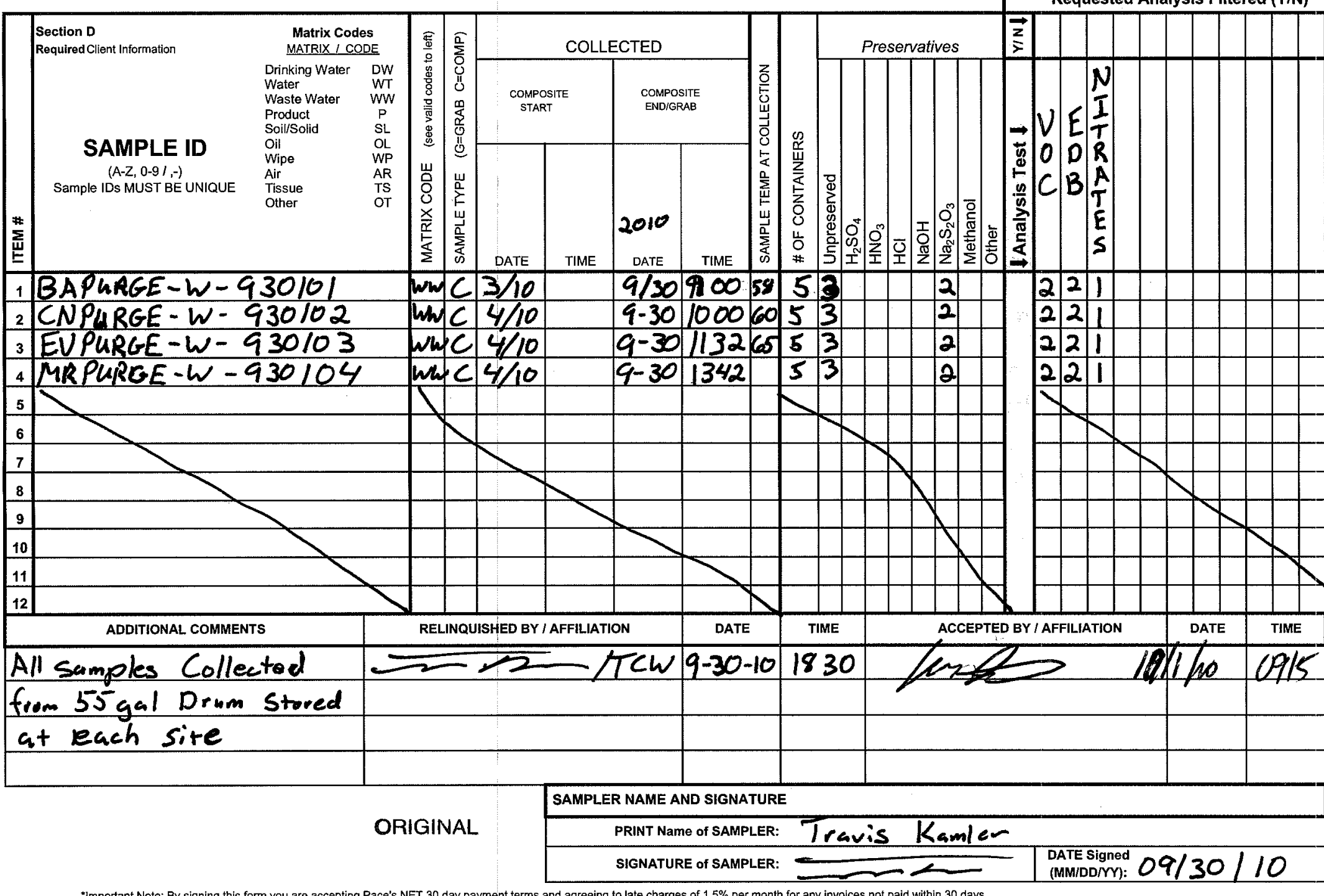

Ceorlebole

$+$


www.pacelabs.com

\section{Sold To:}

Mr. Travis Kamler TCW Construction Inc $141 \mathrm{M}$ Street Lincoln, NE 68508 402-475-5030
Invoice Number: 106081867

Date: $10 / 14 / 2010$

Total Amount Due: $\$ 592.00$

\section{Please Remit To:}

Pace Analytical Services, Inc.

P.O. Box 684056

Milwaukee, WI 53268-4056

\section{Client Number/Client ID 60-508440 / TCW Const}

Client Project: Kansas Waste Water Pace Project No: 6086606

Report Sent To: Mr. David Surgnier,

Comments:

o

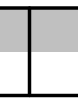

\section{Pace Project Mgr}

Trudy Gipson

\begin{tabular}{|c|c|}
\hline Terms & Page \\
Net 30 Days & \\
\hline
\end{tabular}

Client Name: TCW Construction Inc

Sample Received: 10/1/2010

Mr. Travis Kamler, TCW Construction Inc

\begin{tabular}{|c|c|c|c|c|c|}
\hline \multicolumn{6}{|c|}{ ANALYTICAL CHARGES } \\
\hline Quantity Unit & Description & Method & Matrix & Price & Total \\
\hline $4 \mathrm{Ea}$ & 300.0 IC Anions-Nitrate & EPA 300.0 & Water & $\$ 18.00$ & $\$ 72.00$ \\
\hline $4 \mathrm{Ea}$ & 504 GCS EDB DBCP & EPA 504.1 & Water & $\$ 60.00$ & $\$ 240.00$ \\
\hline \multirow[t]{2}{*}{$4 \mathrm{Ea}$} & 8260 VOC by GC/MS-Full Scan & EPA 5030B/8260 & Water & $\$ 70.00$ & $\$ 280.00$ \\
\hline & & & & Analytical Subtotal & $\$ 592.00$ \\
\hline & & er of Charges 12 & & Total Invoice Amount & $\$ 592.00$ \\
\hline
\end{tabular}

Samples Received for analysis:

\begin{tabular}{lll} 
Lab ID & Client Sample ID & Received \\
\hline 6086606001 & BAPURGE-W-930101 & $10 / 1 / 2010$ 9:15:00 \\
6086606002 & CNPURGE-W-930102 & $10 / 1 / 20109: 15: 00$ \\
6086606003 & EVPURGE-W-930103 & $10 / 1 / 20109: 15: 00$ \\
6086606004 & MRPURGE-W-930104 & $10 / 1 / 20109: 15: 00$
\end{tabular}

If you have any questions or to pay by credit card, please contact Trudy Gipson at Pace.

Phone: 1(913)563-1405 Email: trudy.gipson@pacelabs.com

\section{${ }^{*} 1.5 \%$ MONTHLY FINANCE CHARGE ASSESSED AFTER 30 DAYS OR TERMS OF CONTRACT. PLEASE REFERENCE THE INVOICE NUMBER ON ALL REMITTANCE ADVICE.}

Page 1 of 1

Please complete and return copy of invoice with your payment.

\section{INVOICE TOTAL}

\section{$\$ 592.00$}

\section{Amount Paid: $\quad \$$}

Check No: 


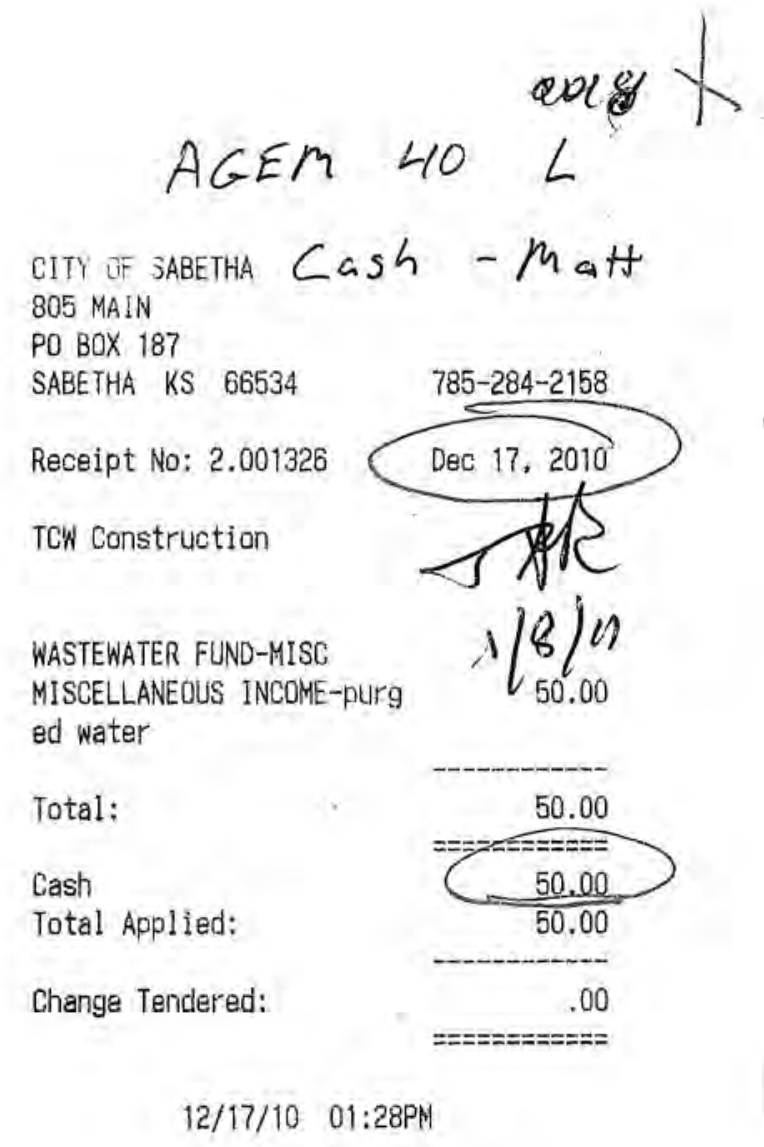




\section{Supplement 2:}

\section{Data Summaries for Verification VOCs Analyses} by TestAmerica Laboratories, Inc. 


\section{TestAmerica}

THE LEADER IN ENYIRONMENTAL TESTING

TestAmerica Laboratories, Inc.

April 28, 2010

Mr. Clyde Dennis

Argonne National Laboratory

9700 S. Cass Avenue

Building 203, Office B149

Argonne, IL 60439

Re: Laboratory Project No. 21005

Case: CNTRALIA; SDG: 136697

Dear Mr. Dennis:

Enclosed are analytical results for samples that were received by TestAmerica Burlington on April $7^{\text {th }}, 2010$. Laboratory identification numbers were assigned, and designated as follows:

$\begin{array}{llll}\text { Lab ID } & \text { Client } & \text { Sample } & \text { Sample } \\ \text { Sample ID } & \text { Date } & \text { Matrix }\end{array}$

Received: 04/07/10 ETR No: 136697

$\begin{array}{llll}825166 & \text { CNMW02-W-27179 } & 04 / 05 / 10 & \text { WATER } \\ 825167 & \text { CNPMP2-W-27181 } & 04 / 05 / 10 & \text { WATER } \\ 825168 & \text { CNPMP3-W-27182 } & 04 / 05 / 10 & \text { WATER } \\ 825169 & \text { CNQCTB-W-27185 } & 04 / 05 / 10 & \text { WATER } \\ 825170 & \text { VHBLK01 } & 04 / 07 / 10 & \text { WATER }\end{array}$

Documentation of the condition of the samples at the time of their receipt and any exception to the laboratory's Sample Acceptance Policy is documented in the Sample Handling section of this submittal. The samples, as received, were not acid preserved. On that basis, the laboratory did provide for the analytical work to be performed within seven days of sample collection.

In order to accommodate field length limitations in processing the data summary forms, the laboratory did, in certain instances, abbreviate the sample identifier. The electronically formatted data provides for the full sample identifier.

\section{SOM01.2 Volatile Organics (Trace Level Water)}

A storage blank was prepared for volatile organics analysis, and stored in association with the storage of the samples. That storage blank, identified as VHBLK01, was carried through the holding period with the samples, and analyzed. 


\section{TestAmerica}

THE LEADER IN ENVIRONMENTAL TESTING

Samples CNMW02-W-27179 and CNPMP2-W-27181 were analyzed at a dilution in order to provide quantification within the range of calibrated instrument response. An additional, more concentrated analysis was performed on each of those samples in order to provide for a lower reporting limit for those compounds that were not identified in the primary analysis. Both sets of results for the analysis of samples CNMW02-W-27179 and CNPMP2-W-27181 are included in this submittal.

Each of the analyses associated with the sample set exhibited an acceptable internal standard performance, and there was an acceptable recovery of each deuterated monitoring compound (DMC) in each analysis. Matrix spike and matrix spike duplicate analyses were not performed on samples in this sample set. Trace concentrations of acetone and 2-butanone were identified in the analysis of each method blank associated with the analytical work. The concentration of each compound in each analysis was below the established reporting limit, and each analysis did meet the technical acceptance criteria for a compliant method blank analysis. Trace concentrations of acetone, 2-butanone, and chloroform were identified in the analysis of the storage blank associated with the sample set. The concentration of each compound in that analysis was below the established reporting limit, and the analysis did meet the technical acceptance criteria for a compliant storage blank analysis. Present in the method blank and storage blank analyses was a non-target constituent that represented a compound that is related to the DMC formulation. The fact that the presence of this compound is not within the laboratory's control is at issue. The derived results for that compound have been qualified with an "X" qualifier to reflect the source of the contamination.

The responses for each of the target analytes met the relative standard deviation criterion in the initial calibration. The response for each target analyte met the percent difference criterion in each continuing calibration check acquisition. The response for each target analyte met the 50.0 percent difference criterion in each closing calibration check acquisition.

The primary quantitation mass for methylcyclohexane that is specified in the Statement of Work is mass 83 . The laboratory did identify a contribution to mass 83 from 1,2-dichloropropane- $d_{6}$, one of the deuterated monitoring compounds (DMCs). The laboratory did change the primary quantitation mass assignment to mass 55 for the quantification of methylcyclohexane.

Manual integration was employed in deriving certain of the analytical results. The values that have been derived from manual integration are qualified on the quantitation reports. Extracted ion current profiles for each manual integration are included in the data package, and further documented in the Sample Preparation section of this submittal.

Any reference within this report to Severn Trent Laboratories, Inc. or STL, should be understood to refer to TestAmerica Laboratories, Inc. (formerly known as Severn Trent Laboratories, Inc.) The analytical results associated with the samples presented in this test report were generated under a quality system that adheres to requirements specified in the NELAC standard. Release of the data in this test report and any associated electronic deliverables is authorized by the Laboratory Director's designee as verified by the following signature. 


\section{TestAmerica}

THE LEADER IN ENVIRONMENTAL TESTING

If there are any questions regarding this submittal, please contact me at 802 660-1990.

Sincerely,

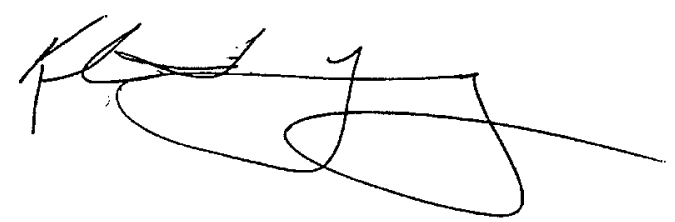

Kirk F. Young

Project Manager

KFY/hsf

Enclosure 
FedEx \# 855876829668

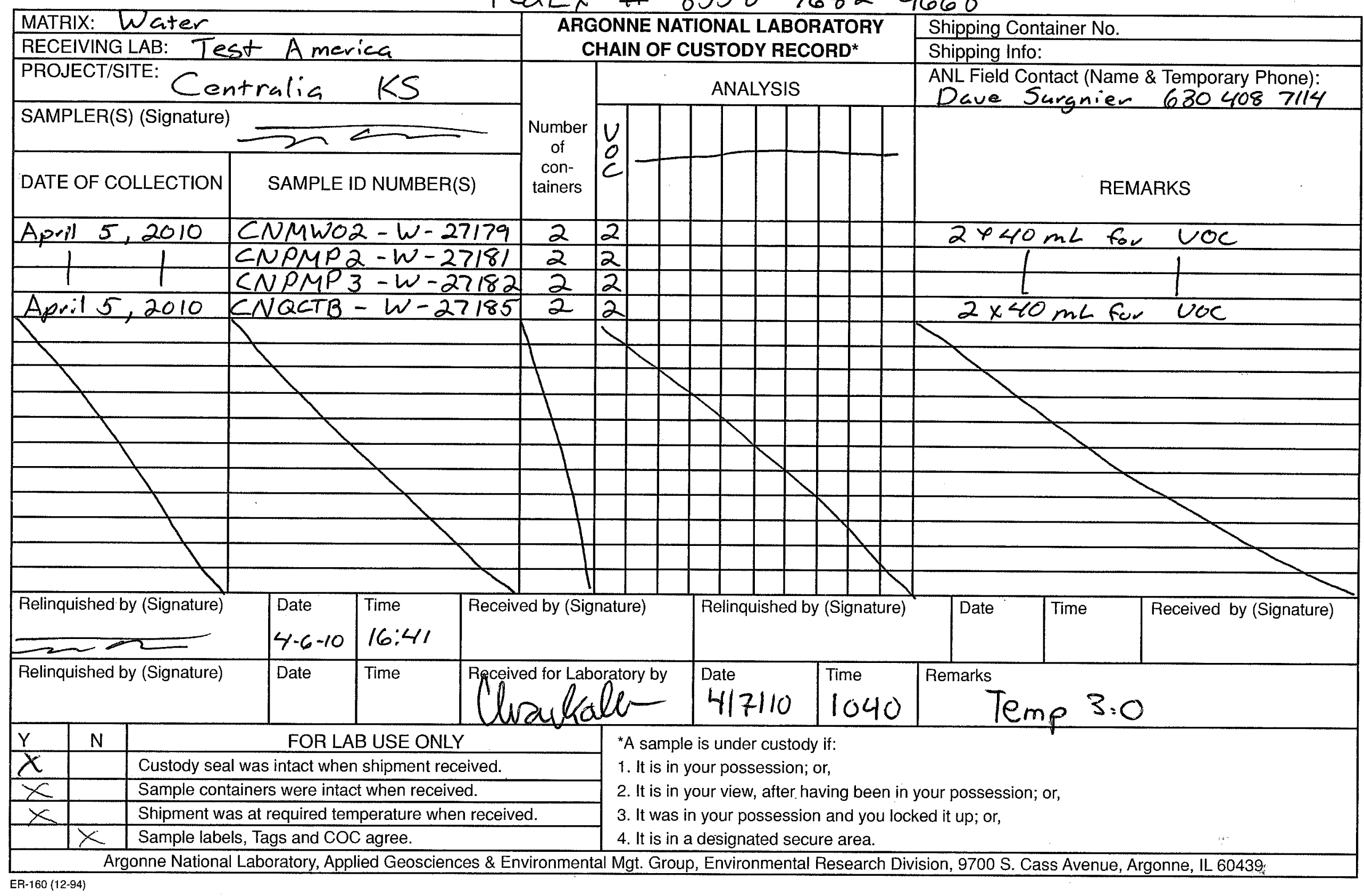




\section{TestAmerica}

THE LEADER IN ENVIRONMENTAL TESTING

\section{Sample Data Summary - SOM01.2 Volatiles - Trace}


$1 A$ - FORM I VOA-1

VOLATILE ORGANICS ANALYSIS DATA SHEET
EPA SAMPLE NO.

MW02W27179

Lab Name: TESTAMERICA BURLINGTON

Contract: $8 \mathrm{E}-00302$

Lab Code: STLV Case No.: CNTRALIA Mod. Ref No.:

SDG NO.: 136697

Matrix: (SOIL/SED/WATER) Water

Sample wt/vol: $25.0 \quad(\mathrm{~g} / \mathrm{mL}) \mathrm{mL}$

Level : (TRACE/LOW/MED) TRACE

$\%$ Moisture: not dec.

GC Column: DB-624

Soil Extract Volume:

ID $: 0.53$

(mm)

(UL)

(mL)
Lab Sample ID: 825166

Lab File ID: 825166D2

Date Received: 04/07/2010

Date Analyzed: 04/09/2010

Dilution Factor: 11.0

Soil Aliquot Volume:

(uL)

\begin{tabular}{|c|c|c|c|}
\hline CAS NO. & COMPOUND & $\begin{array}{l}\text { CONCENTRATION UNITS: } \\
(\mathrm{ug} / \mathrm{L} \text { or } \mathrm{ug} / \mathrm{kg}) \mathrm{ug} / \mathrm{L}\end{array}$ & $\mathrm{Q}$ \\
\hline$==========$ & 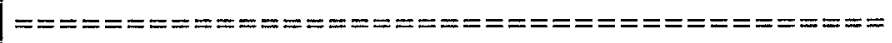 & 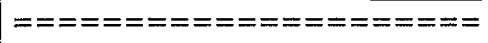 & $========$ \\
\hline $75-71-8$ & Dichlorodifluoromethane & 5.5 & $\mathrm{U}$ \\
\hline $74-87-3$ & Chloromethane & 5.5 & $\mathrm{U}$ \\
\hline $75-01-4$ & Vinyl chloride & 5.5 & $\mathrm{U}$ \\
\hline $74-83-9$ & Bromomethane & 5.5 & $\mathrm{U}$ \\
\hline $75-00-3$ & Chloroethane & 5.5 & $\mathrm{U}$ \\
\hline $75-69-4$ & Trichlorofluoromethane & 5.5 & $\mathrm{U}$ \\
\hline $75-35-4$ & 1,1-Dichloroethene & 5.5 & $\mathrm{U}$ \\
\hline $76-13-1$ & 1,1,2-Trichloro-1,2,2-trifluoroethane & 5.5 & $\mathrm{U}$ \\
\hline $67-64-1$ & Acetone & 55 & $\mathrm{U}$ \\
\hline $75-15-0$ & Carbon disulfide & 5.5 & $\mathrm{U}$ \\
\hline $79-20-9$ & Methyl acetate & 5.5 & $\mathrm{U}$ \\
\hline $75-09-2$ & Methylene chloride & 5.5 & $\mathrm{U}$ \\
\hline $156-60-5$ & trans-1,2-Dichloroethene & 5.5 & $\mathrm{U}$ \\
\hline $1634-04-4$ & Methyl tert-butyl ether & 5.5 & $\mathrm{U}$ \\
\hline $75-34-3$ & 1,1-Dichloroethane & 5.5 & $\mathrm{U}$ \\
\hline $156-59-2$ & cis-1,2-Dichloroethene & 5.5 & $\mathrm{U}$ \\
\hline $78-93-3$ & 2-Butanone & 55 & $\mathrm{U}$ \\
\hline $74-97-5$ & Bromochloromethane & 5.5 & $\mathrm{U}$ \\
\hline $67-66-3$ & Chloroform & 5.5 & $\mathrm{U}$ \\
\hline $71-55-6$ & 1,1,1-Trichloroethane & 5.5 & $\mathrm{U}$ \\
\hline $110-82-7$ & Cyclohexane & 5.5 & $\mathrm{U}$ \\
\hline $56-23-5$ & Carbon tetrachloride & 5.5 & $\mathrm{U}$ \\
\hline $71-43-2$ & Benzene & 5.5 & $\mathrm{U}$ \\
\hline $107-06-2$ & 1,2-Dichloroethane & 5.5 & $\mathrm{U}$ \\
\hline
\end{tabular}

Report 1,4-Dioxane for Low-Medium VOA analysis only 
IB - FORM I VOA-2

VOLATILE ORGANICS ANALYSIS DATA SHEET
EPA SAMPLE NO.

MWO2W27179
Lab Name: TESTAMERICA BURLINGTON

Lab Code: STLV

Case No.: CNTRALIA

Mod

Matrix: (SOIL/SED/WATER) Water

Sample wt/vol: $25.0 \quad(\mathrm{~g} / \mathrm{mL}) \mathrm{mL}$

Level: (TRACE/LOW/MED) TRACE

$\%$ Moisture: not dec.

GC Column: DB -624

Soil Extract Volume:

ID : 0.53

(mm)

(uL)

Contract: 8E-00302

SDG No.: 136697
Lab Sample ID: 825166

Lab File ID: $825166 \mathrm{D} 2$

Date Received: 04/07/2010

Date Analyzed: 04/09/2010

Dilution Factor: 11.0

Soil Aliquot Volume:

(uL)
Purge Volume: 25.0

(mL)

\begin{tabular}{|c|c|c|c|}
\hline CAS NO. & COMPOUND & $\begin{array}{l}\text { CONCENTRATION UNITS : } \\
(\mathrm{ug} / \mathrm{L} \text { or } \mathrm{ug} / \mathrm{kg}) \mathrm{ug} / \mathrm{L}\end{array}$ & $Q$ \\
\hline$=========$ & 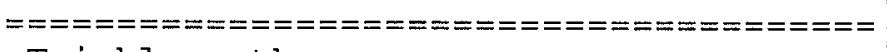 & 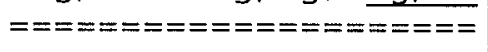 & $== \pm====$ \\
\hline $79-01-6$ & Trichloroethene & 5.5 & $\mathrm{U}$ \\
\hline $108-87-2$ & Methylcyclohexane & 5.5 & $\mathrm{U}$ \\
\hline $78-87-5$ & 1,2 -Dichloropropane & 5.5 & $\mathrm{U}$ \\
\hline $75-27-4$ & Bromodichloromethane & 5.5 & $\mathrm{U}$ \\
\hline $10061-01-5$ & cis-1,3-Dichloropropene & 5.5 & $\mathrm{U}$ \\
\hline $108-10-1$ & 4-Methyl-2-pentanone & 55 & $\mathrm{U}$ \\
\hline $108-88-3$ & Toluene & 940 & $E$ \\
\hline $10061-02-6$ & trans-1,3-Dichloropropene & 5.5 & $\mathrm{U}$ \\
\hline $79-00-5$ & $1,1,2$-Trichloroethane & 5.5 & $\mathrm{U}$ \\
\hline $127-18-4$ & Tetrachloroethene & 5.5 & $\mathrm{U}$ \\
\hline $591-78-6$ & 2-Hexanone & 55 & $\mathrm{U}$ \\
\hline $124-48-1$ & Dibromochloromethane & 5.5 & $\mathrm{U}$ \\
\hline $106-93-4$ & 1,2-Dibromoethane & 5.5 & $\mathrm{U}$ \\
\hline $108-90-7$ & Chlorobenzene & 5.5 & $\mathrm{U}$ \\
\hline $100-41-4$ & Ethylbenzene & 25 & \\
\hline $95-47-6$ & o-Xylene & 5.5 & $\overline{\mathrm{U}}$ \\
\hline $179601-23-1$ & $\mathrm{~m}, \mathrm{p}$-Xylene & 5.5 & $\mathrm{U}$ \\
\hline $100-42-5$ & Styrene & 5.5 & $\mathrm{U}$ \\
\hline $75-25-2$ & Bromoform & 5.5 & $\mathrm{U}$ \\
\hline $98-82-8$ & Isopropylbenzene & 5.5 & $\mathrm{U}$ \\
\hline $79-34-5$ & $1,1,2,2$-Tetrachloroethane & 5.5 & $\mathrm{U}$ \\
\hline $541-73-1$ & 1,3-Dichlorobenzene & 5.5 & $\mathrm{U}$ \\
\hline $106-46-7$ & 1,4-Dichlorobenzene & 5.5 & $\mathrm{U}$ \\
\hline $95-50-1$ & 1,2-Dichlorobenzene & 5.5 & $\mathrm{U}$ \\
\hline $96-12-8$ & 1,2-Dibromo-3-chloropropane & 5.5 & $\mathrm{U}$ \\
\hline $120-82-1$ & 1,2,4-Trichlorobenzene & 5.5 & $\mathrm{U}$ \\
\hline $87-61-6$ & $1,2,3$-Trichlorobenzene & 5.5 & $\mathrm{U}$ \\
\hline
\end{tabular}


IJ - FORM I VOA-TIC

VOLATILE ORGANICS ANALYSIS DATA SHEET TENTATIVELY IDENTIFIED COMPOUNDS
EPA SAMPLE NO.

MW02W2 7179
Lab Name: TESTAMERICA BURLINGTON

Lab Code: STLV Case No.: CNTRALIA

Matrix: (SOIL/SED/WATER) water

Sample wt/vol: $25.0 \quad(\mathrm{~g} / \mathrm{mL}) \mathrm{mL}$

Level: (TRACE or LOW/MED) TRACE

$\%$ Moisture: not dec.

GC Column: DB-624

Soil Extract Volume:

ID $: 0.53$

CONCENTRATION UNITS: (ug/L or $\mathrm{ug} / \mathrm{kg}) \mathrm{ug} / \mathrm{L}$
Contract: $8 \mathrm{E}-00302$

Mod. Ref No.:

SDG NO.: 136697
Lab Sample ID: 825166

Lab File ID: 825166D2

Date Received: 04/07/2010

Date Analyzed: 04/09/2010

(mm) Dilution Factor: 11.0

(uL) Soil Aliquot Volume:

(uL)

Purge Volume: 25.0

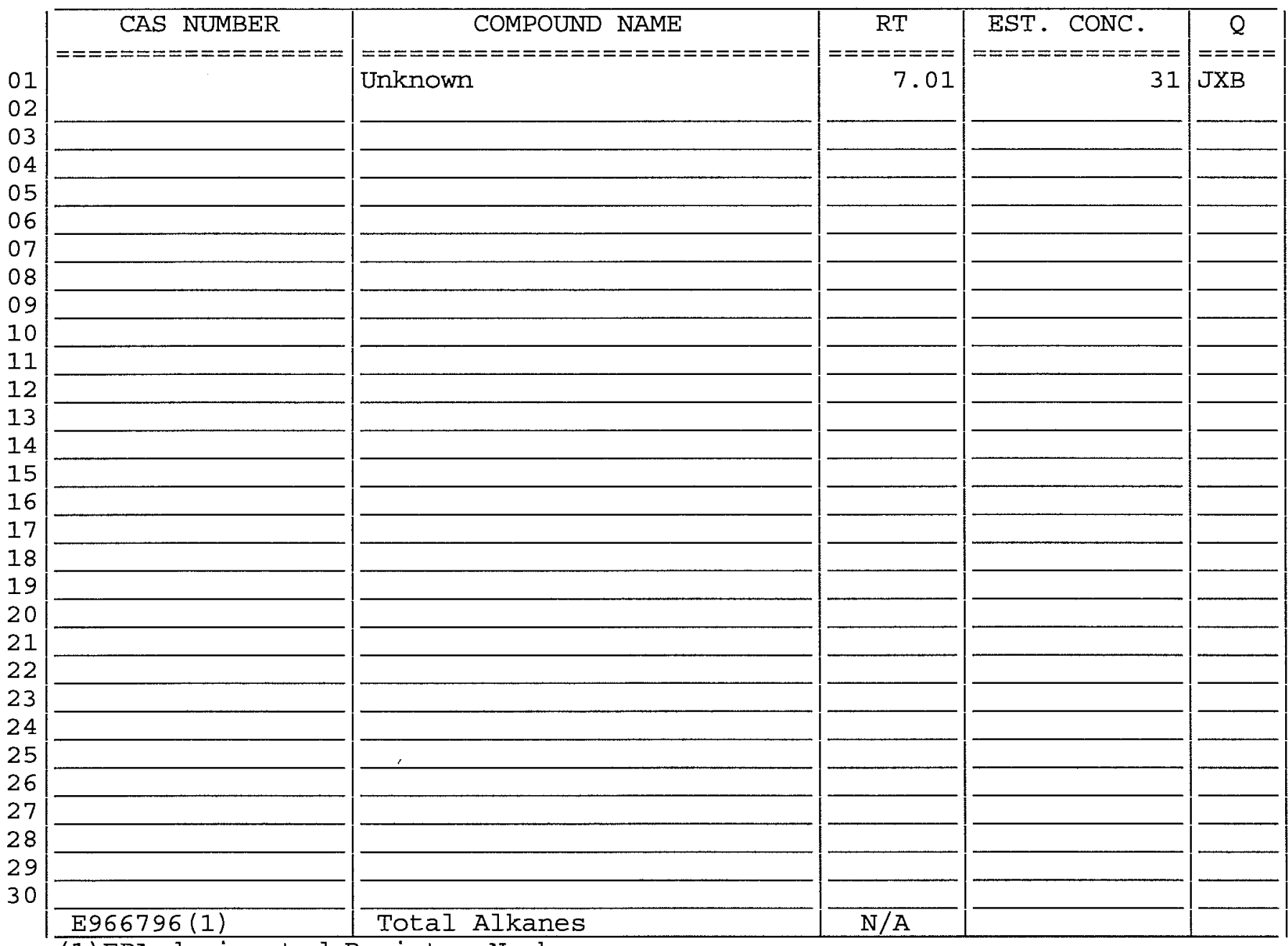

(1) EPA-designated Registry Number. 
$1 A$ - FORM I VOA-1

VOLATILE ORGANICS ANALYSIS DATA SHEET
EPA SAMPLE NO.

MW02W27179DL
Lab Name: TESTAMERICA BURLINGTON

Lab Code: STLV Case No.: CNTRALIA Mod. Ref No.:
Contract: $8 \mathrm{E}-00302$
Matrix: (SOIL/SED/WATER) Water

Sample wt/vol: 25.0

$(\mathrm{g} / \mathrm{mL}) \mathrm{mL}$

Level: (TRACE/LOW/MED) TRACE

$\%$ Moisture: not dec.

GC Column: DB-624

ID : 0.53

Soil Extract Volume:

Purge Volume: 25.0
Lab Sample ID: 825166D1

Lab File ID: $825166 \mathrm{D}$

Date Received: 04/07/2010

Date Analyzed: 04/09/2010

Dilution Factor: 129.4

Soil Aliquot Volume:

(mL)

\begin{tabular}{|c|c|c|c|}
\hline CAS NO. & COMPOUND & $\begin{array}{l}\text { CONCENTRATION UNITS: } \\
(\mathrm{ug} / \mathrm{L} \text { or } \mathrm{ug} / \mathrm{kg}) \mathrm{ug} / \mathrm{L}\end{array}$ & $\mathrm{Q}$ \\
\hline$==========$ & 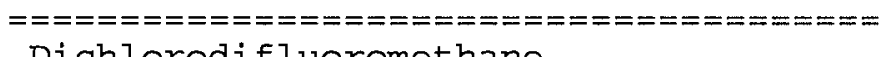 & 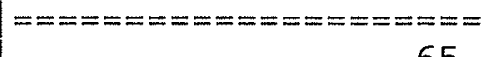 & $======$ \\
\hline $75-71-8$ & Dichlorodifluoromethane & 65 & $\mathrm{U}$ \\
\hline $74-87-3$ & Chloromethane & 65 & $\mathrm{U}$ \\
\hline $75-01-4$ & Vinyl chloride & 65 & $\mathrm{U}$ \\
\hline $74-83-9$ & Bromomethane & 65 & $\mathrm{U}$ \\
\hline $75-00-3$ & Chloroethane & 65 & $\mathrm{U}$ \\
\hline $75-69-4$ & Trichlorofluoromethane & 65 & $\mathrm{U}$ \\
\hline $75-35-4$ & 1,1-Dichloroethene & 65 & $\mathrm{U}$ \\
\hline $76-13-1$ & $1,1,2$-Trichloro-1,2,2-trifluoroethane & 65 & $\mathrm{U}$ \\
\hline $67-64-1$ & Acetone & 170 & DJB \\
\hline $75-15-0$ & Carbon disulfide & 65 & $\mathrm{U}$ \\
\hline $79-20-9$ & Methyl acetate & 65 & $\mathrm{U}$ \\
\hline $75-09-2$ & Methylene chloride & 65 & $\mathrm{U}$ \\
\hline $156-60-5$ & trans $-1,2-\mathrm{Dichloroethene}$ & 65 & $\mathrm{U}$ \\
\hline $1634-04-4$ & Methyl tert-butyl ether & 65 & $\mathrm{U}$ \\
\hline $75-34-3$ & 1,1-Dichloroethane & 65 & $\mathrm{U}$ \\
\hline $156-59-2$ & Cis-1,2-Dichloroethene & 65 & $\mathrm{U}$ \\
\hline $78-93-3$ & 2-Butanone & 650 & $\mathrm{U}$ \\
\hline $74-97-5$ & Bromochloromethane & 65 & $\mathrm{U}$ \\
\hline $67-66-3$ & Chloroform & 55 & DJ \\
\hline $71-55-6$ & $1,1,1$-Trichloroethane & 65 & $\mathrm{U}$ \\
\hline $110-82-7$ & Cyclohexane & 65 & $\mathrm{U}$ \\
\hline $56-23-5$ & Carbon tetrachloride & 65 & $\mathrm{U}$ \\
\hline $71-43-2$ & Benzene & 65 & $\mathrm{U}$ \\
\hline $107-06-2$ & 1,2-Dichloroethane & 65 & $\mathrm{U}$ \\
\hline
\end{tabular}

Report 1,4-Dioxane for Low-Medium VOA analysis only 
1B - FORM I VOA-2

VOLATILE ORGANICS ANALYSIS DATA SHEET
EPA SAMPLE NO.

MW02W2 7179DL
Lab Name: TESTAMERICA BURLINGTON

Contract : $8 \mathrm{E}-00302$

Lab Code: STLV Case No.: CNTRALIA Mod. Ref No.:

SDG No.: 136697

Matrix: (SOIL/SED/WATER) water

Sample wt/vol: 25.0

(g/mL $\mathrm{mL}$

Level : (TRACE/LOW/MED) TRACE

\% Moisture: not dec.

GC Column: DB-624

Soil Extract Volume:

ID $: 0.53$

(mm)

(uL)

(mL)

Purge Volume: 25.0
Lab Sample ID: 825166D1

Lab File ID: $825166 \mathrm{D}$

Date Received: 04/07/2010

Date Analyzed: 04/09/2010

Dilution Factor: 129.4

Soil Aliquot Volume:

(uL)

\begin{tabular}{|c|c|c|c|}
\hline CAS NO. & COMPOUND & $\begin{array}{l}\text { CONCENTRATION UNITS: } \\
(\mathrm{ug} / \mathrm{L} \text { or } \mathrm{ug} / \mathrm{kg}) \mathrm{ug} / \mathrm{L}\end{array}$ & $Q$ \\
\hline$=== \pm=====-=$ & 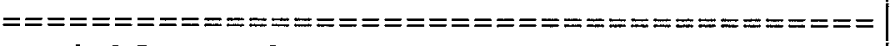 & 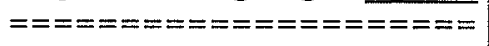 & $======$ \\
\hline $79-01-6$ & Trichloroethene & 65 & $\mathrm{U}$ \\
\hline $108-87-2$ & Methylcyclohexane & 65 & $\mathrm{U}$ \\
\hline $78-87-5$ & 1,2-Dichloropropane & 65 & $\mathrm{U}$ \\
\hline $75-27-4$ & Bromodichloromethane & 65 & $\mathrm{U}$ \\
\hline $10061-01-5$ & cis-1,3-Dichloropropene & 65 & $\mathrm{U}$ \\
\hline $108-10-1$ & 4-Methyl-2-pentanone & 650 & $\mathrm{U}$ \\
\hline $108-88-3$ & Toluene & 1600 & $\mathrm{D}$ \\
\hline $10061-02-6$ & trans-1,3-Dichloropropene & 65 & $\mathrm{U}$ \\
\hline $79-00-5$ & 1,1,2-Trichloroethane & 65 & $\mathrm{U}$ \\
\hline $127-18-4$ & Tetrachloroethene & 65 & $\mathrm{U}$ \\
\hline $591-78-6$ & 2-Hexanone & 650 & $\mathrm{U}$ \\
\hline $124-48-1$ & Dibromochloromethane & 65 & $\mathrm{U}$ \\
\hline $106-93-4$ & 1,2-Dibromoethane & 65 & $\mathrm{U}$ \\
\hline $108-90-7$ & Chlorobenzene & 65 & $\mathrm{U}$ \\
\hline $100-41-4$ & Ethylbenzene & 27 & DJ \\
\hline $95-47-6$ & o-Xylene & 65 & $\mathrm{U}$ \\
\hline $179601-23-1$ & $\mathrm{~m}, \mathrm{p}-\mathrm{xy}$ lene & 65 & $\mathrm{U}$ \\
\hline $100-42-5$ & Styrene & 65 & $\mathrm{U}$ \\
\hline $75-25-2$ & Bromoform & 65 & $\mathrm{U}$ \\
\hline $98-82-8$ & Isopropylbenzene & 65 & $\mathrm{U}$ \\
\hline $79-34-5$ & $1,1,2,2$-Tetrachloroethane & 65 & $\mathrm{U}$ \\
\hline $541-73-1$ & 1,3-Dichlorobenzene & 65 & $\mathrm{U}$ \\
\hline $106-46-7$ & 1,4-Dichlorobenzene & 65 & $\mathrm{U}$ \\
\hline $95-50-1$ & 1,2-Dichlorobenzene & 65 & $\mathrm{U}$ \\
\hline $96-12-8$ & 1,2-Dibromo-3-chloropropane & 65 & $\mathrm{U}$ \\
\hline $120-82-1$ & $1,2,4$-Trichlorobenzene & 65 & $\mathrm{U}$ \\
\hline $87-61-6$ & 1,2,3-Trichlorobenzene & 65 & $\mathrm{U}$ \\
\hline
\end{tabular}


IJ - FORM I VOA-TIC

VOLATILE ORGANICS ANALYSIS DATA SHEET TENTATIVELY IDENTIFIED COMPOUNDS
EPA SAMPLE NO.

MW02W2 7179DL
Lab Name: TESTAMERICA BURLINGTON

Lab Code: STLV Case No.: CNTRALIA

Matrix: (SOIL/SED/WATER) Water

Sample wt/vol: $25.0 \quad(\mathrm{~g} / \mathrm{mL}) \mathrm{mL}$

Level: (TRACE or LOW/MED) TRACE

\% Moisture: not dec.

GC Column: DB-624

ID $: 0.53$

Soil Extract Volume:

CONCENTRATION UNITS: (ug/L or ug/kg) ug/L
Contract: $8 E-00302$

Mod. Ref No.:

SDG NO.: 136697
Lab sample ID: 825166D1

Lab File ID: 825166D

Date Received: 04/07/2010

Date Analyzed: 04/09/2010

(mm) Dilution Factor: 129.4

(uL) Soil Aliquot Volume:

(UL)

Purge Volume: 25.0

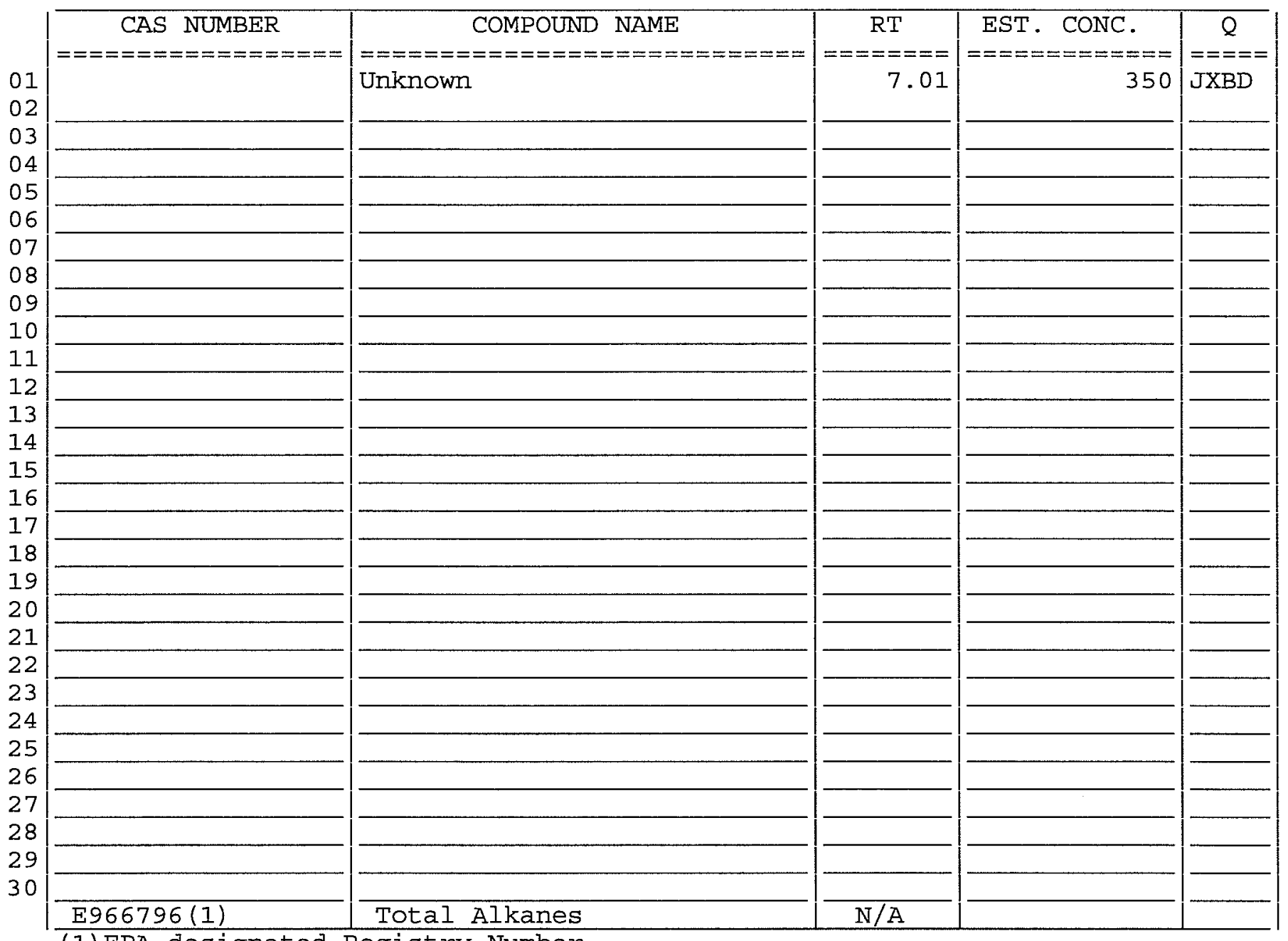

(1) EPA-designated Registry Number. 
$1 A$ - FORM I VOA-1

VOLATILE ORGANICS ANALYSIS DATA SHEET
EPA SAMPLE NO.

PMP2W2 7181
Lab Name: TESTAMERICA BURLINGTON

Lab Code: STLV

Case No.: CNTRALIA

Matrix: (SOIL/SED/WATER) Water

Sample wt/vol: 25.0

$(\mathrm{g} / \mathrm{mL}) \mathrm{mL}$

Level: (TRACE/LOW/MED) TRACE

\% Moisture: not dec.

GC Column: DB-624

ID : 0.53

Soil Extract Volume:

Purge Volume: 25.0
Contract : 8E-00302

SDG No.: 136697
Lab Sample ID: 825167

Lab File ID: 825167D2

Date Received: 04/07/2010

Date Analyzed: 04/08/2010

Dilution Factor: 4.0

Soil Aliquot Volume:

(uL)

\begin{tabular}{|c|c|c|c|}
\hline CAS NO. & COMPOUND & $\begin{array}{l}\text { CONCENTRATION UNITS: } \\
(\mathrm{ug} / \mathrm{L} \text { or } \mathrm{ug} / \mathrm{kg}) \mathrm{ug} / \mathrm{L}\end{array}$ & Q \\
\hline$==========$ & 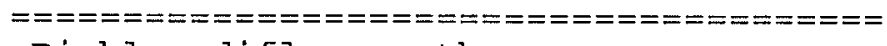 & 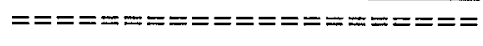 & $======-$ \\
\hline $75-71-8$ & Dichlorodifluoromethane & 2.0 & $\mathrm{U}$ \\
\hline $74-87-3$ & Chloromethane & 2.0 & $\mathrm{U}$ \\
\hline $75-01-4$ & Vinyl chloride & 2.0 & $\mathrm{U}$ \\
\hline $74-83-9$ & Bromomethane & 2.0 & U \\
\hline $75-00-3$ & Chloroethane & 2.0 & U \\
\hline $75-69-4$ & Trichlorofluoromethane & 2.0 & $\mathrm{U}$ \\
\hline $75-35-4$ & 1,1-Dichloroethene & 2.0 & $\mathrm{U}$ \\
\hline $76-13-1$ & 1,1,2-Trichloro-1,2,2-trifluoroethane & 2.0 & $\mathrm{U}$ \\
\hline $67-64-1$ & Acetone & 100 & $\mathrm{~B}$ \\
\hline $75-15-0$ & Carbon disulfide & 1.0 & J \\
\hline $79-20-9$ & Methyl acetate & 2.0 & $\mathrm{U}$ \\
\hline $75-09-2$ & Methylene chloride & 4.3 & \\
\hline $156-60-5$ & trans-1,2-Dichloroethene & 2.0 & $\mathrm{U}$ \\
\hline $1634-04-4$ & Methyl tert-butyl ether & 2.0 & $\mathrm{U}$ \\
\hline $75-34-3$ & 1,1-Dichloroethane & 2.0 & $U$ \\
\hline $156-59-2$ & Cis-1,2-Dichloroethene & 2.0 & $\mathrm{U}$ \\
\hline $78-93-3$ & 2-Butanone & 68 & $\mathrm{~B}$ \\
\hline $74-97-5$ & Bromochloromethane & 2.0 & $\mathrm{U}$ \\
\hline $67-66-3$ & Chloroform & 120 & $\mathrm{E}$ \\
\hline $71-55-6$ & $1,1,1$-Trichloroethane & 2.0 & $U$ \\
\hline $110-82-7$ & Cyclohexane & 2.0 & $\mathrm{U}$ \\
\hline $56-23-5$ & Carbon tetrachloride & 690 & $\mathrm{E}$ \\
\hline $71-43-2$ & Benzene & 2.0 & $\mathrm{U}$ \\
\hline $107-06-2$ & 1,2-Dichloroethane & 2.0 & $\mathrm{U}$ \\
\hline
\end{tabular}

Report 1,4-Dioxane for Low-Medium VOA analysis only 
$1 B$ - FORM I VOA-2

VOLATILE ORGANICS ANALYSIS DATA SHEET
EPA SAMPLE NO.

PMP2W27181

Lab Name: TESTAMERICA BURLINGTON

Contract : $8 E-00302$

Lab Code: STLV Case No.: CNTRALIA Mod. Ref No.:

SDG No.: 136697

Matrix: (SOIL/SED/WATER) Water

Sample wt/vol: $25.0 \quad(\mathrm{~g} / \mathrm{mL}) \mathrm{mL}$

Level : (TRACE/LOW/MED) TRACE

\% Moisture: not dec.

GC Column: DB-624

Soil Extract Volume:

ID $: 0.53$

(mm)

(uL)

(mL)

Purge Volume: 25.0
Lab Sample ID: 825167

Lab File ID: 825167D2

Date Received: 04/07/2010

Date Analyzed: 04/08/2010

Dilution Factor: 4.0

Soil Aliquot Volume:

(uL) $\left(\frac{10}{20}\right.$

\begin{tabular}{|c|c|c|c|}
\hline CAS NO. & COMPOUND & $\begin{array}{l}\text { CONCENTRATION UNITS: } \\
(\mathrm{ug} / \mathrm{L} \text { or } \mathrm{ug} / \mathrm{kg}) \mathrm{ug} / \mathrm{L}\end{array}$ & Q \\
\hline$===========$ & 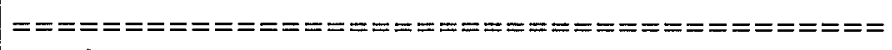 & $=============== \pm====0$ & $======x=$ \\
\hline $79-01-6$ & Trichloroethene & 2.0 & $\mathrm{U}$ \\
\hline $108-87-2$ & Methylcyclohexane & 2.0 & $\mathrm{U}$ \\
\hline $78-87-5$ & 1,2-Dichloropropane & 2.0 & $\mathrm{U}$ \\
\hline $75-27-4$ & Bromodichloromethane & 2.0 & $\mathrm{U}$ \\
\hline $10061-01-5$ & cis-1,3-Dichloropropene & 2.0 & $\mathrm{U}$ \\
\hline $108-10-1$ & 4-Methyl-2-pentanone & 20 & $\mathrm{U}$ \\
\hline $108-88-3$ & Toluene & 290 & $\mathrm{E}$ \\
\hline $10061-02-6$ & trans-1,3-Dichloropropene & 2.0 & $\mathrm{U}$ \\
\hline $79-00-5$ & 1,1,2-Trichloroethane & 2.0 & $\mathrm{U}$ \\
\hline $127-18-4$ & Tetrachloroethene & 2.0 & $\mathrm{U}$ \\
\hline $591-78-6$ & 2-Hexanone & 20 & $\mathrm{U}$ \\
\hline $124-48-1$ & Dibromochloromethane & 2.0 & $\mathrm{U}$ \\
\hline $106-93-4$ & 1,2-Dibromoethane & 2.0 & $\mathrm{U}$ \\
\hline $108-90-7$ & Chlorobenzene & 2.0 & $\mathrm{U}$ \\
\hline $100-41-4$ & Ethylbenzene & 2.0 & $\mathrm{U}$ \\
\hline $95-47-6$ & o-xylene & 2.0 & $\mathrm{U}$ \\
\hline $179601-23-1$ & $\mathrm{~m}, \mathrm{p}$-xylene & 2.0 & $\mathrm{U}$ \\
\hline $100-42-5$ & styrene & 2.0 & $\mathrm{U}$ \\
\hline $75-25-2$ & Bromoform & 2.0 & $\mathrm{U}$ \\
\hline $98-82-8$ & Isopropylbenzene & 2.0 & $\mathrm{U}$ \\
\hline $79-34-5$ & $1,1,2,2$-Tetrachloroethane & 2.0 & $\mathrm{U}$ \\
\hline $541-73-1$ & 1,3-Dichlorobenzene & 2.0 & $\mathrm{U}$ \\
\hline $106-46-7$ & 1,4-Dichlorobenzene & 2.0 & $\mathrm{U}$ \\
\hline $95-50-1$ & 1,2-Dichlorobenzene & 2.0 & $\mathrm{U}$ \\
\hline $96-12-8$ & 1,2-Dibromo-3-chloropropane & 2.0 & $\mathrm{U}$ \\
\hline $120-82-1$ & 1,2,4-Trichlorobenzene & 2.0 & $\mathrm{U}$ \\
\hline $87-61-6$ & 1,2,3-Trichlorobenzene & 2.0 & $\mathrm{U}$ \\
\hline
\end{tabular}


$1 \mathrm{~J}$ - FORM I VOA-TIC

VOLATILE ORGANICS ANALYSIS DATA SHEET TENTATIVELY IDENTIFIED COMPOUNDS
EPA SAMPLE NO.

PMP2W27181
Lab Name: TESTAMERICA BURLINGTON

Lab Code: STLV Case No.: CNTRALIA

Matrix: (SOIL/SED/WATER) Water

Sample wt/vol: $25.0 \quad(\mathrm{~g} / \mathrm{mL}) \mathrm{mL}$

Level: (TRACE or LOW/MED) TRACE

\% Moisture: not dec.

GC Column: DB-624

Soil Extract Volume:

ID : 0.53

CONCENTRATION UNITS: $(\mathrm{ug} / \mathrm{L}$ or $\mathrm{ug} / \mathrm{kg}) \mathrm{ug} / \mathrm{L}$
Contract: $8 \mathrm{E}-00302$

Mod. Ref No.:

SDG No.: 136697

Lab Sample ID: 825167

Lab File ID: 825167D2

Date Received: 04/07/2010

Date Analyzed: 04/08/2010

(mm) Dilution Factor: 4.0

(uL) Soil Aliquot Volume:

(uL)

Purge Volume: 25.0

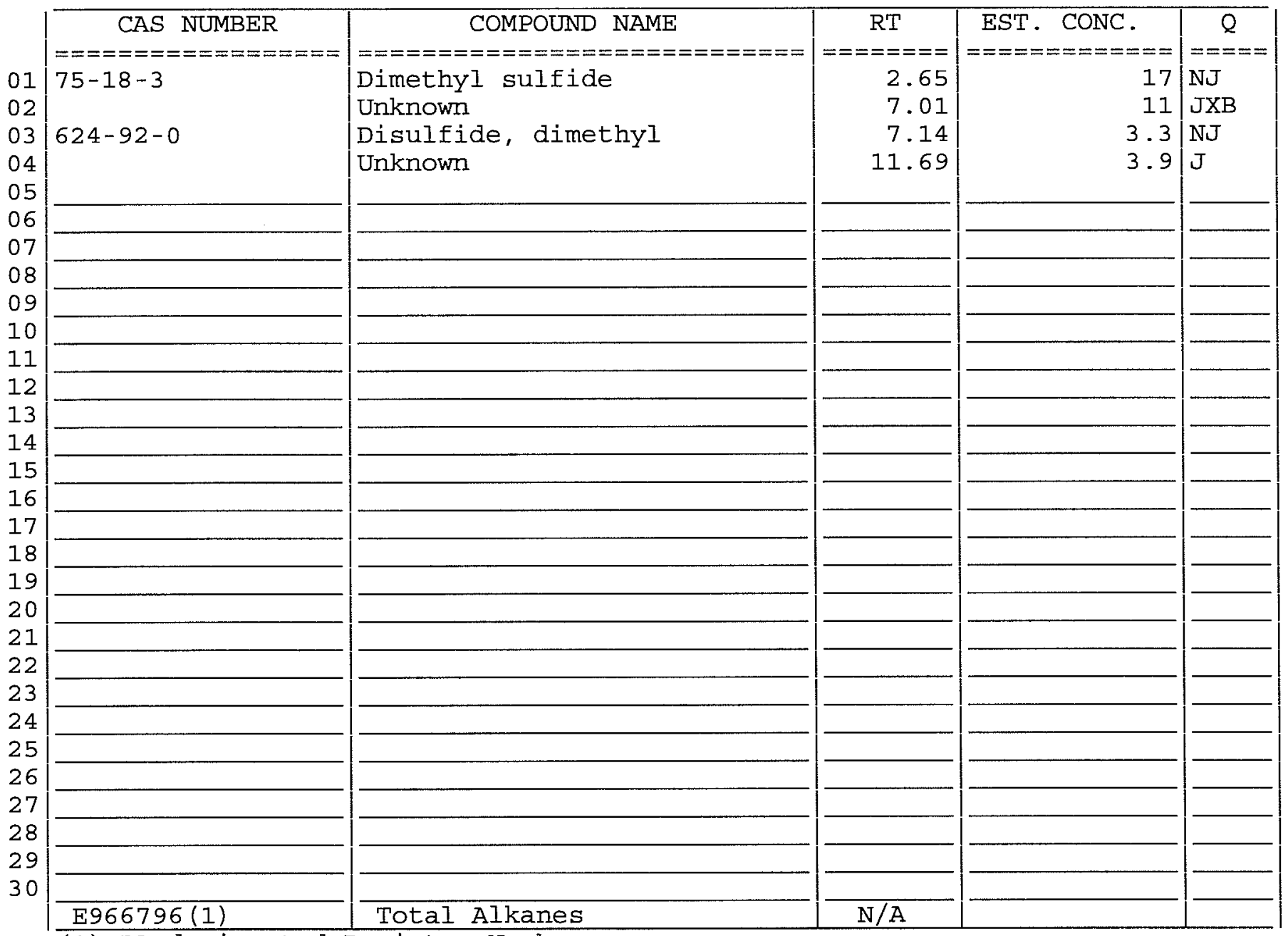

(1) EPA-designated Registry Number. 
$1 A$ - FORM I VOA-1

VOLATILE ORGANICS ANALYSIS DATA SHEET

Lab Name: TESTAMERICA BURLINGTON

Contract: $8 \mathrm{E}-00302$

Lab Code: STLV Case No.: CNTRALIA Mod. Ref No.:

Lab Sample ID: 825167D1

Matrix: (SOIL/SED/WATER) Water

Lab File ID: 825167D

Sample wt/vol: 25.0

$(\mathrm{g} / \mathrm{mL}) \mathrm{mL}$

Level : (TRACE/LOW/MED) TRACE

\%oisture: not dec.

Date Received: 04/07/2010

Date Analyzed: 04/08/2010

GC Column: DB-624

ID $: 0.53$

$(\mathrm{mm})$

Dilution Factor: 46.3

Soil Extract Volume:

(uL)

Soil Aliquot Volume:

(uL)
EPA SAMPLE NO.

PMP2W27181DL

Purge Volume: 25.0

$(\mathrm{mL})$

\begin{tabular}{|c|c|c|c|}
\hline CAS NO. & COMPOUND & $\begin{array}{l}\text { CONCENTRATION UNITS: } \\
(\mathrm{ug} / \mathrm{L} \text { or } \mathrm{ug} / \mathrm{kg}) \mathrm{ug} / \mathrm{L}\end{array}$ & Q \\
\hline$===========$ & 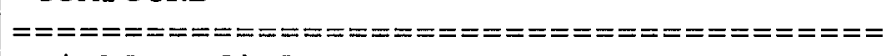 & $============ \pm=====$ & $=======$ \\
\hline $75-71-8$ & Dichlorodifluoromethane & 23 & $\mathrm{U}$ \\
\hline $74-87-3$ & Chloromethane & 23 & $\mathrm{U}$ \\
\hline $75-01-4$ & Vinyl chloride & 23 & $\mathrm{U}$ \\
\hline $74-83-9$ & Bromomethane & 23 & $\mathrm{U}$ \\
\hline $75-00-3$ & Chloroethane & 23 & $\mathrm{U}$ \\
\hline $75-69-4$ & Trichlorofluoromethane & 23 & $\mathrm{U}$ \\
\hline $75-35-4$ & 1,1-Dichloroethene & 23 & $\mathrm{U}$ \\
\hline $76-13-1$ & 1,1,2-Trichloro-1,2,2-trifluoroethane & 23 & $\mathrm{U}$ \\
\hline $67-64-1$ & Acetone & 140 & DJB \\
\hline $75-15-0$ & Carbon disulfide & 23 & $\mathrm{U}$ \\
\hline $79-20-9$ & Methyl acetate & 23 & $\mathrm{U}$ \\
\hline $75-09-2$ & Methylene chloride & 23 & $\mathrm{U}$ \\
\hline $156-60-5$ & trans-1,2-Dichloroethene & 23 & $\mathrm{U}$ \\
\hline $1634-04-4$ & Methyl tert-butyl ether & 23 & $\mathrm{U}$ \\
\hline $75-34-3$ & 1,1-Dichloroethane & 23 & $\mathrm{U}$ \\
\hline $156-59-2$ & cis-1,2-Dichloroethene & 23 & $\mathrm{U}$ \\
\hline $78-93-3$ & 2-Butanone & 80 & DJB \\
\hline $74-97-5$ & Bromochloromethane & 23 & $\mathrm{U}$ \\
\hline $67-66-3$ & Chloroform & 130 & D \\
\hline $71-55-6$ & 1,1,1-Trichloroethane & 23 & $\mathrm{U}$ \\
\hline $110-82-7$ & Cyclohexane & 23 & $\mathrm{U}$ \\
\hline $56-23-5$ & Carbon tetrachloride & 670 & D \\
\hline $71-43-2$ & Benzene & 23 & $\mathrm{U}$ \\
\hline $107-06-2$ & 1,2-Dichloroethane & 23 & $\mathrm{U}$ \\
\hline
\end{tabular}

Report 1,4-Dioxane for Low-Medium VOA analysis only 


\section{$1 B$ - FORM I VOA-2 \\ VOLATILE ORGANICS ANALYSIS DATA SHEET}

Lab Name: TESTAMERICA BURLINGTON

Contract: $8 \mathrm{E}-00302$

Lab Code: STLV Case No.: CNTRALIA Mod. Ref No.:

Lab Sample ID : 825167D1

Matrix: (SOIL/SED/WATER) Water

Sample wt/vol: $25.0 \quad(\mathrm{~g} / \mathrm{mL}) \mathrm{mL}$

Lab File ID: $825167 D$

Level: (TRACE/LOW/MED) TRACE

$\%$ Moisture: not dec.

Date Received: 04/07/2010

Date Analyzed: 04/08/2010

GC Column: DB-624

ID $: 0.53$

(mm)

Dilution Factor: 46.3

Soil Extract Volume:

(uL)

Soil Aliquot Volume:

(uL)

Purge Volume: 25.0

(mL)

\begin{tabular}{|c|c|c|c|}
\hline CAS NO. & COMPOUND & $\begin{array}{l}\text { CONCENTRATION UNITS: } \\
(\mathrm{ug} / \mathrm{L} \text { or } u g / \mathrm{kg}) \mathrm{ug} / \mathrm{L}\end{array}$ & Q \\
\hline$==========$ & 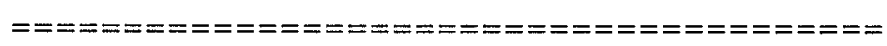 & $============ \pm=====$ & $======$ \\
\hline $79-01-6$ & Trichloroethene & 23 & $\mathrm{U}$ \\
\hline $108-87-2$ & Methylcyclohexane & 23 & $\mathrm{U}$ \\
\hline $78-87-5$ & 1,2-Dichloropropane & 23 & $\mathrm{U}$ \\
\hline $75-27-4$ & Bromodichloromethane & 23 & $\mathrm{U}$ \\
\hline $10061-01-5$ & cis-1,3-Dichloropropene & 23 & $\mathrm{U}$ \\
\hline $108-10-1$ & 4-Methyl-2-pentanone & 230 & $\mathrm{U}$ \\
\hline $108-88-3$ & Toluene & 390 & $\mathrm{D}$ \\
\hline $10061-02-6$ & trans-1,3-Dichloropropene & 23 & $\mathrm{U}$ \\
\hline $79-00-5$ & 1,1,2-Trichloroethane & 23 & $\mathrm{U}$ \\
\hline $127-18-4$ & Tetrachloroethene & 23 & $\mathrm{U}$ \\
\hline $591-78-6$ & 2-Hexanone & 230 & $\mathrm{U}$ \\
\hline $124-48-1$ & Dibromochloromethane & 23 & $\mathrm{U}$ \\
\hline $106-93-4$ & 1,2-Dibromoethane & 23 & $\mathrm{U}$ \\
\hline $108-90-7$ & Chlorobenzene & 23 & $\mathrm{U}$ \\
\hline $100-41-4$ & Ethylbenzene & 23 & $\mathrm{U}$ \\
\hline $95-47-6$ & o-xylene & 23 & $\mathrm{U}$ \\
\hline $179601-23-1$ & $\mathrm{~m}, \mathrm{p}$-xylene & 23 & $\mathrm{U}$ \\
\hline $100-42-5$ & styrene & 23 & $\mathrm{U}$ \\
\hline $75-25-2$ & Bromoform & 23 & $\mathrm{U}$ \\
\hline $98-82-8$ & Isopropylbenzene & 23 & $\mathrm{U}$ \\
\hline $79-34-5$ & $1,1,2,2$-Tetrachloroethane & 23 & $\mathrm{U}$ \\
\hline $541-73-1$ & 1,3-Dichlorobenzene & 23 & $\mathrm{U}$ \\
\hline $106-46-7$ & 1,4-Dichlorobenzene & 23 & $\mathrm{U}$ \\
\hline $95-50-1$ & 1,2-Dichlorobenzene & 23 & $\mathrm{U}$ \\
\hline $96-12-8$ & 1,2-Dibromo-3-chloropropane & 23 & $\mathrm{U}$ \\
\hline $120-82-1$ & $1,2,4$-Trichlorobenzene & 23 & $\mathrm{U}$ \\
\hline $87-61-6$ & $1,2,3$-Trichlorobenzene & 23 & $\mathrm{U}$ \\
\hline
\end{tabular}


IJ - FORM I VOA-TIC

VOLATILE ORGANICS ANALYSIS DATA SHEET TENTATIVELY IDENTIFIED COMPOUNDS
EPA SAMPLE NO. PMP2W27181DL
Lab Name: TESTAMERICA BURLINGTON

Lab Code: STLV Case No.: CNTRALIA

Matrix: (SOIL/SED/WATER) Water

Sample wt/vol: $25.0 \quad(\mathrm{~g} / \mathrm{mL}) \mathrm{mL}$

Level: (TRACE or LOW/MED) TRACE

\% Moisture: not dec.

GC Column: DB-624

ID $: 0.53$

Soil Extract Volume:

CONCENTRATION UNITS: (ug/L or ug/kg) ug/L
Contract: $8 E-00302$

Mod. Ref No.:

SDG No. : 136697

Lab Sample ID: $825167 D 1$

Lab File ID: $825167 D$

Date Received: 04/07/2010

Date Analyzed: 04/08/2010

(mm) Dilution Factor: 46.3

(uL) Soil Aliquot Volume:

(uL)

Purge Volume: 25.0

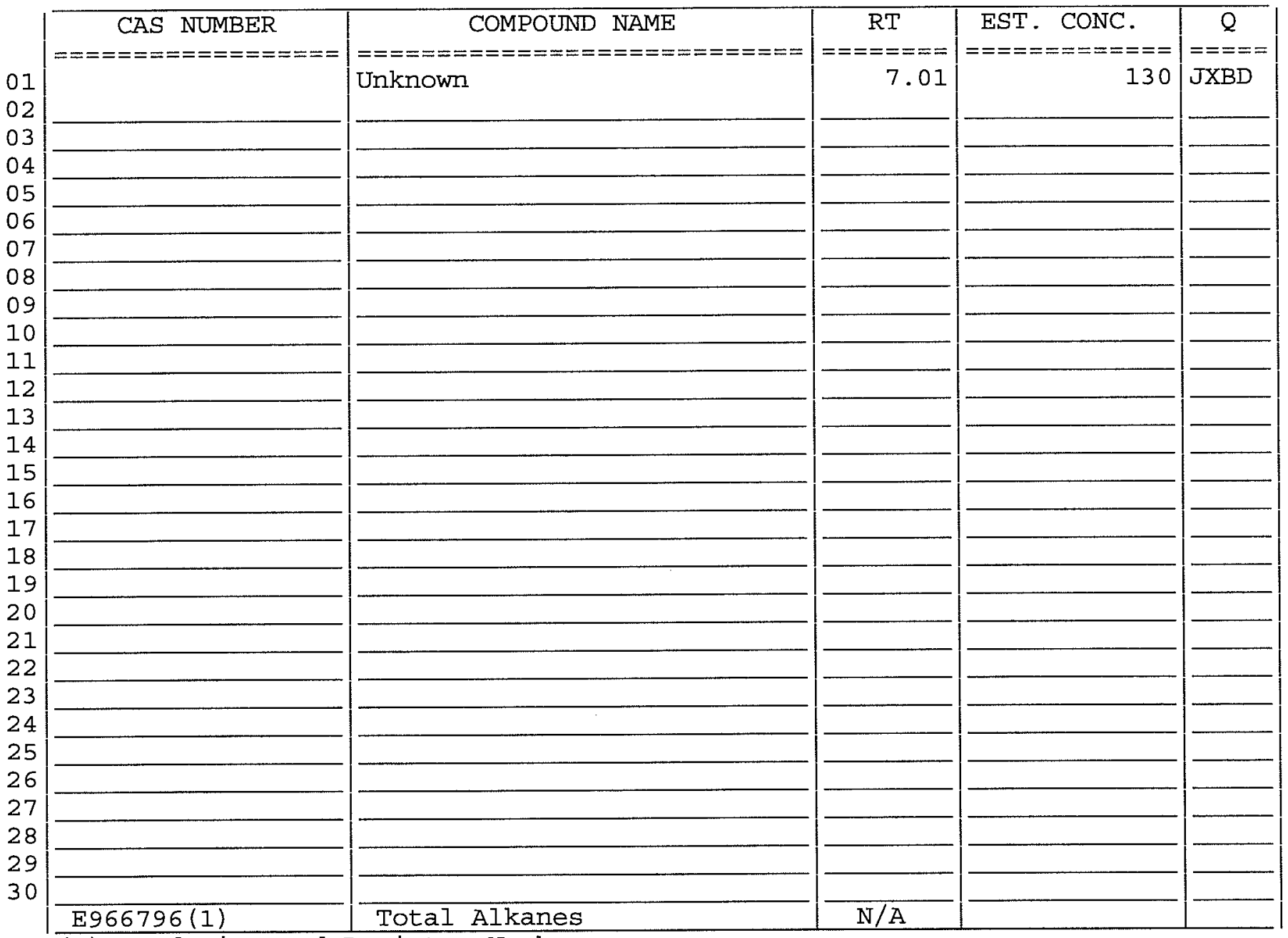

(1) EPA-designated Registry Number. 
$1 A$ - FORM I VOA-1

VOLATILE ORGANICS ANALYSIS DATA SHEET
EPA SAMPLE NO.

PMP3W2 7182

Lab Name: TESTAMERICA BURLINGTON

Contract: $8 \mathrm{E}-00302$

Lab Code: STLV Case No.: CNTRALIA Mod. Ref No.:

SDG No.: 136697

Matrix: (SOIL/SED/WATER) Water

Sample wt/vol: 25.0

$(\mathrm{g} / \mathrm{mL}) \mathrm{mL}$

Level: (TRACE/LOW/MED) TRACE

\% Moisture: not dec.

GC Column: DB-624

Soil Extract Volume:

ID $: 0.53$

(mm)

(uL)

(mL)

Purge Volume: 25.0
Lab Sample ID: 825168

Lab File ID: 825168

Date Received: 04/07/2010

Date Analyzed: 04/08/2010

Dilution Factor: 1.0

Soil Aliquot Volume:

(uL)

\begin{tabular}{|c|c|c|c|}
\hline CAS NO. & COMPOUND & $\begin{array}{l}\text { CONCENTRATION UNITS: } \\
(\mathrm{ug} / \mathrm{L} \text { or } \mathrm{ug} / \mathrm{kg} \text { ) } \mathrm{ug} / \mathrm{L}\end{array}$ & Q \\
\hline$===========$ & 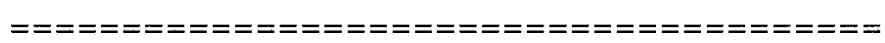 & $=============0=\overline{===0}$ & $=======$ \\
\hline $75-71-8$ & Dichlorodifluoromethane & 0.50 & $\mathrm{U}$ \\
\hline $74-87-3$ & Chloromethane & 0.50 & $\mathrm{U}$ \\
\hline $75-01-4$ & Vinyl chloride & 0.50 & U \\
\hline $74-83-9$ & Bromomethane & 0.50 & $\mathrm{U}$ \\
\hline $75-00-3$ & Chloroethane & 0.50 & $\mathrm{U}$ \\
\hline $75-69-4$ & Trichlorofluoromethane & 0.50 & $\mathrm{U}$ \\
\hline $75-35-4$ & 1,1-Dichloroethene & 0.50 & $\mathrm{U}$ \\
\hline $76-13-1$ & 1,1,2-Trichloro-1,2,2-trifluoroethane & 0.50 & $\mathrm{U}$ \\
\hline $67-64-1$ & Acetone & 1.1 & JB \\
\hline $75-15-0$ & Carbon disulfide & 0.50 & $\mathrm{U}$ \\
\hline $79-20-9$ & Methyl acetate & 0.50 & $\mathrm{U}$ \\
\hline $75-09-2$ & Methylene chloride & 0.50 & $\mathrm{U}$ \\
\hline $156-60-5$ & trans-1,2-Dichloroethene & 0.50 & $\mathrm{U}$ \\
\hline $1634-04-4$ & Methyl tert-butyl ether & 0.50 & $\mathrm{U}$ \\
\hline $75-34-3$ & 1,1-Dichloroethane & 0.50 & $\mathrm{U}$ \\
\hline $156-59-2$ & cis-1,2-Dichloroethene & 0.50 & $\mathrm{U}$ \\
\hline $78-93-3$ & 2-Butanone & 0.52 & JB \\
\hline $74-97-5$ & Bromochloromethane & 0.50 & $\mathrm{U}$ \\
\hline $67-66-3$ & Chloroform & 0.21 & $\mathrm{~J}$ \\
\hline $71-55-6$ & 1,1,1-Trichloroethane & 0.50 & $\mathrm{U}$ \\
\hline $110-82-7$ & Cyclohexane & 0.50 & $\mathrm{U}$ \\
\hline $56-23-5$ & Carbon tetrachloride & 0.50 & $\mathrm{U}$ \\
\hline $71-43-2$ & Benzene & 0.50 & $\mathrm{U}$ \\
\hline $107-06-2$ & 1,2-Dichloroethane & 0.50 & U \\
\hline
\end{tabular}

Report 1,4-Dioxane for Low-Medium VOA analysis only 
1B - FORM I VOA-2

VOLATILE ORGANICS ANALYSIS DATA SHEET
EPA SAMPLE NO.

PMP3W27182

Lab Name: TESTAMERICA BURLINGTON

Contract: $8 \mathrm{E}-00302$

Lab Code: STLV Case No.: CNTRALIA Mod. Ref No.:

SDG No. : 136697

Matrix: (SOIL/SED/WATER) water

Sample wt/vol: 25.0

$(\mathrm{g} / \mathrm{mL}) \mathrm{mL}$

Level: (TRACE/LOW/MED) TRACE

\% Moisture: not dec.

GC Column: DB-624

ID : 0.53

(mm)

(uL)

(mL)

Purge Volume: 25.0
Lab Sample ID: 825168

Lab File ID: 825168

Date Received: 04/07/2010

Date Analyzed: 04/08/2010

Dilution Factor: 1.0

Soil Aliquot Volume:

(uL)

\begin{tabular}{|c|c|c|c|}
\hline CAS NO. & COMPOUND & $\begin{array}{l}\text { CONCENTRATION UNITS: } \\
(\mathrm{ug} / \mathrm{L} \text { or } \mathrm{ug} / \mathrm{kg}) \mathrm{ug} / \mathrm{L}\end{array}$ & Q \\
\hline$====== \pm=====$ & 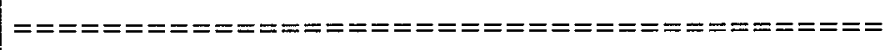 & 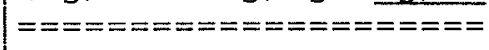 & $=======$ \\
\hline $79-01-6$ & Trichloroethene & 0.50 & $\mathrm{U}$ \\
\hline $108-87-2$ & Methylcyclohexane & 0.50 & $\mathrm{U}$ \\
\hline $78-87-5$ & 1,2-Dichloropropane & 0.50 & $\mathrm{U}$ \\
\hline $75-27-4$ & Bromodichloromethane. & 0.50 & $\mathrm{U}$ \\
\hline $10061-01-5$ & Cis-1,3-Dichloropropene & 0.50 & $\mathrm{U}$ \\
\hline $108-10-1$ & 4-Methyl-2-pentanone & 5.0 & $\mathrm{U}$ \\
\hline $108-88-3$ & Toluene & 3.7 & \\
\hline $10061-02-6$ & trans $-1,3$-Dichloropropene & 0.50 & $\mathrm{U}$ \\
\hline $79-00-5$ & 1,1,2-Trichloroethane & 0.50 & $\mathrm{U}$ \\
\hline $127-18-4$ & Tetrachloroethene & 0.50 & $\mathrm{U}$ \\
\hline $591-78-6$ & 2-Hexanone & 5.0 & $\mathrm{U}$ \\
\hline $124-48-1$ & Dibromochloromethane & 0.50 & $\mathrm{U}$ \\
\hline $106-93-4$ & 1,2-Dibromoethane & 0.50 & $\mathrm{U}$ \\
\hline $108-90-7$ & Chlorobenzene & 0.50 & U \\
\hline $100-41-4$ & Ethylbenzene & 0.50 & $\mathrm{U}$ \\
\hline $95-47-6$ & o-Xylene & 0.50 & U \\
\hline $179601-23-1$ & $\mathrm{~m}, \mathrm{p}$-Xylene & 0.40 & $\mathrm{~J}$ \\
\hline $100-42-5$ & styrene & 0.50 & $\mathrm{U}$ \\
\hline $75-25-2$ & Bromoform & 0.50 & U \\
\hline $98-82-8$ & Isopropylbenzene & 0.50 & $\mathrm{U}$ \\
\hline $79-34-5$ & $1,1,2,2$-Tetrachloroethane & 0.50 & $\mathrm{U}$ \\
\hline $541-73-1$ & 1,3-Dichlorobenzene & 0.50 & $\mathrm{U}$ \\
\hline $106-46-7$ & 1,4-Dichlorobenzene & 0.50 & $\mathrm{U}$ \\
\hline $95-50-1$ & 1,2-Dichlorobenzene & 0.50 & $\mathrm{U}$ \\
\hline $96-12-8$ & 1,2-Dibromo-3-chloropropane & 0.50 & U \\
\hline $120-82-1$ & 1,2,4-Trichlorobenzene & 0.50 & $\mathrm{U}$ \\
\hline $87-61-6$ & 1,2,3-Trichlorobenzene & 0.50 & $\mathrm{U}$ \\
\hline
\end{tabular}


IJ - FORM I VOA-TIC

VOLATILE ORGANICS ANALYSIS DATA SHEET

TENTATIVELY IDENTIFIED COMPOUNDS
EPA SAMPLE NO.

PMP3W27182
Lab Name: TESTAMERICA BURLINGTON

Lab Code: STLV Case No.: CNTRALIA

Matrix: (SOIL/SED/WATER) Water

Sample wt/vol: $25.0 \quad(\mathrm{~g} / \mathrm{mL}) \mathrm{mL}$

Level: (TRACE or LOW/MED) TRACE

\% Moisture: not dec.

GC Column: DB-624

Soil Extract Volume:

ID $: 0.53$

CONCENTRATION UNITS: $(\mathrm{ug} / \mathrm{L}$ or $\mathrm{ug} / \mathrm{kg}) \mathrm{ug} / \mathrm{L}$
Contract: $8 \mathrm{E}-00302$

Mod. Ref No.:

SDG No.: 136697
Lab Sample ID: 825168

Lab File ID: 825168

Date Received: 04/07/2010

Date Analyzed: 04/08/2010

(mm) Dilution Factor: 1.0

(uL) Soil Aliquot Volume:

(uL)

Purge Volume: 25.0

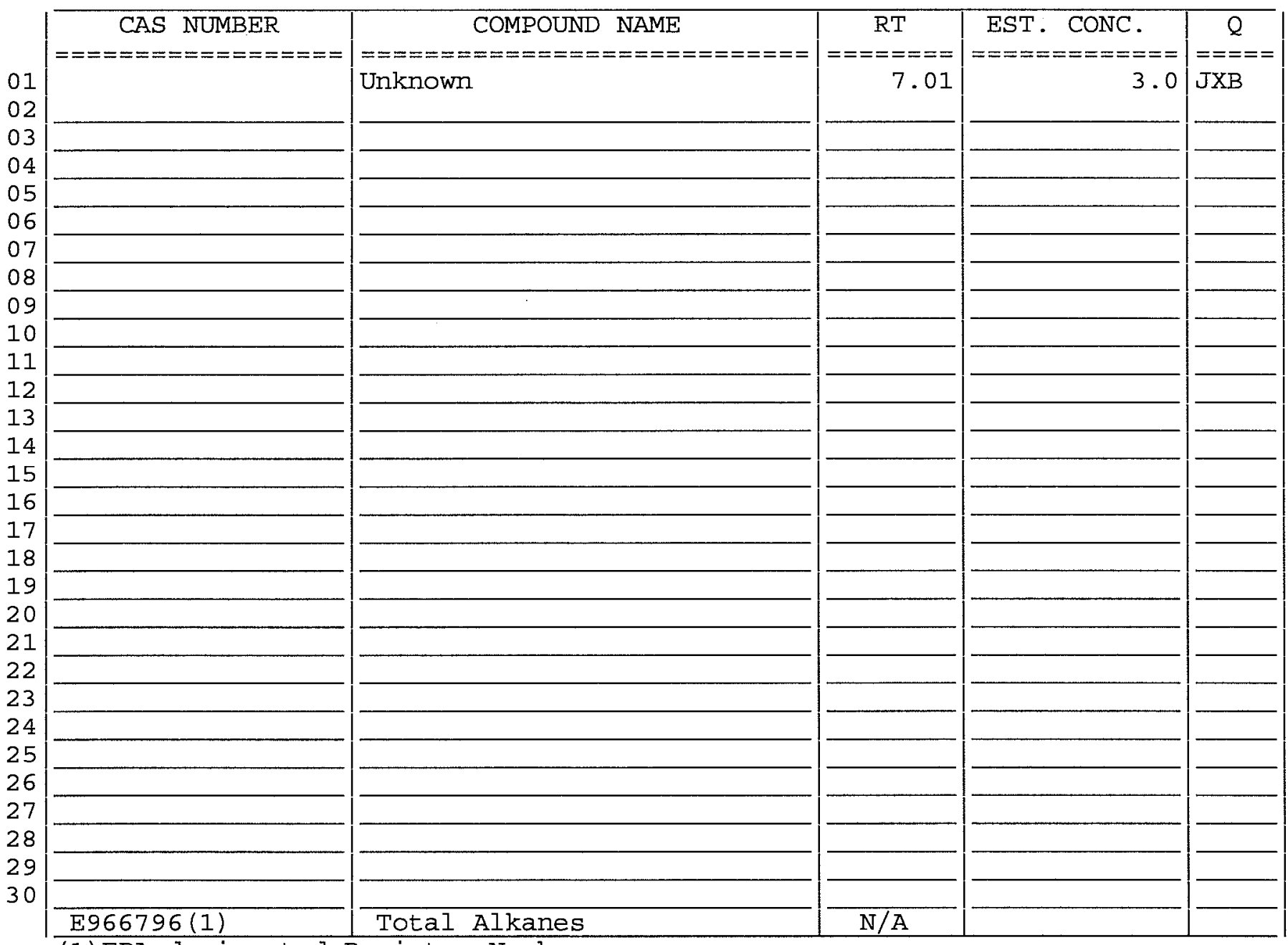

(1) EPA-designated Registry Number. 
IA - FORM I VOA-1

VOLATILE ORGANICS ANALYSIS DATA SHEET
EPA SAMPLE NO.

QCTBW27185

Lab Name: TESTAMERICA BURLINGTON

Contract: $8 E-00302$

Lab Code: STLV Case No.: CNTRALIA Mod. Ref No.:

SDG No.: 136697

Matrix: (SOIL/SED/WATER) Water

Sample wt/vol: $25.0 \quad(\mathrm{~g} / \mathrm{mL}) \mathrm{mL}$

Level: (TRACE/LOW/MED) TRACE

\% Moisture: not dec.

GC Column: DB-624

Soil Extract Volume:

Purge Volume: 25.0
Lab Sample ID: 825169

Lab File ID: 825169

Date Received: 04/07/2010

Date Analyzed: 04/08/2010

Dilution Factor: 1.0

(uL) Soil Aliquot Volume: (uL)

(mL)

\begin{tabular}{|c|c|c|c|}
\hline CAS NO. & COMPOUND & $\begin{array}{l}\text { CONCENTRATION UNITS: } \\
(\mathrm{ug} / \mathrm{L} \text { or } \mathrm{ug} / \mathrm{kg}) \mathrm{ug} / \mathrm{L}\end{array}$ & Q \\
\hline$=== \pm=== \pm===$ & 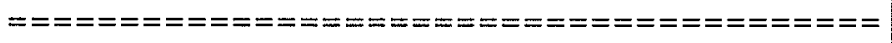 & 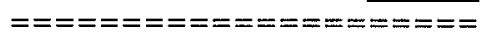 & $=======$ \\
\hline $75-71-8$ & Dichlorodifluoromethane & 0.50 & $\mathrm{U}$ \\
\hline $74-87-3$ & Chloromethane & 0.50 & $\mathrm{U}$ \\
\hline $75-01-4$ & Vinyl chloride & 0.50 & $\mathrm{U}$ \\
\hline $74-83-9$ & Bromomethane & 0.50 & $\mathrm{U}$ \\
\hline $75-00-3$ & Chloroethane & 0.50 & $\mathrm{U}$ \\
\hline $75-69-4$ & Trichlorofluoromethane & 0.50 & $\mathrm{U}$ \\
\hline $75-35-4$ & 1,1-Dichloroethene & 0.50 & $\mathrm{U}$ \\
\hline $76-13-1$ & 1,1,2-Trichloro-1,2,2-trifluoroethane & 0.50 & $\mathrm{U}$ \\
\hline $67-64-1$ & Acetone & 8.4 & B \\
\hline $75-15-0$ & Carbon disulfide & 0.50 & $\mathrm{U}$ \\
\hline $79-20-9$ & Methyl acetate & 0.50 & U \\
\hline $75-09-2$ & Methylene chloride & 0.50 & $\mathrm{U}$ \\
\hline $156-60-5$ & trans-1,2-Dichloroethene & 0.50 & $\mathrm{U}$ \\
\hline $1634-04-4$ & Methyl tert-butyl ether & 0.50 & $\mathrm{U}$ \\
\hline $75-34-3$ & 1,1-Dichloroethane & 0.50 & $\mathrm{U}$ \\
\hline $156-59-2$ & cis-1,2-Dichloroethene & 0.50 & $\mathrm{U}$ \\
\hline $78-93-3$ & 2-Butanone & 1.2 & JB \\
\hline $74-97-5$ & Bromochloromethane & 0.50 & $\mathrm{U}$ \\
\hline $67-66-3$ & Chloroform & 0.50 & U \\
\hline $71-55-6$ & 1,1,1-Trichloroethane & 0.50 & $\mathrm{U}$ \\
\hline $110-82-7$ & Cyclohexane & 0.50 & $\mathrm{U}$ \\
\hline $56-23-5$ & Carbon tetrachloride & 0.50 & $\mathrm{U}$ \\
\hline $71-43-2$ & Benzene & 0.34 & $\mathrm{~J}$ \\
\hline $107-06-2$ & 1,2-Dichloroethane & 0.50 & $\mathrm{U}$ \\
\hline
\end{tabular}

Report 1,4-Dioxane for Low-Medium VOA analysis only 
$1 B$ - FORM I VOA-2

VOLATILE ORGANICS ANALYSIS DATA SHEET
EPA SAMPLE NO.

QCTBW27185

Lab Name: TESTAMERICA BURLINGTON

Contract: $8 \mathrm{E}-00302$

Lab Code: STLV Case No.: CNTRAIIA Mod. Ref No.:

SDG No. : 136697

Matrix: (SOIL/SED/WATER) water

Sample wt/vol: $25.0 \quad(\mathrm{~g} / \mathrm{mL}) \mathrm{mL}$

Level: (TRACE/LOW/MED) TRACE

\% Moisture: not dec.

GC Column: DB-624

Soil Extract Volume:

ID : 0.53

$(\mathrm{mm})$

(uL)

(mL)

Purge Volume: 25.0
Lab Sample ID: 825169

Lab File ID: 825169

Date Received: 04/07/2010

Date Analyzed: 04/08/2010

Dilution Factor: 1.0

Soil Aliquot Volume:

(uL)

\begin{tabular}{|c|c|c|c|}
\hline CAS NO. & COMPOUND & $\begin{array}{l}\text { CONCENTRATION UNITS: } \\
(\mathrm{ug} / \mathrm{L} \text { or } \mathrm{ug} / \mathrm{kg}) \mathrm{ug} / \mathrm{L}\end{array}$ & $\mathrm{Q}$ \\
\hline$==========$ & 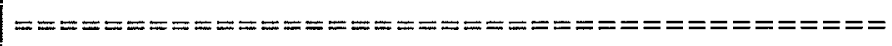 & 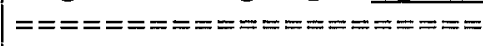 & $======$ \\
\hline $79-01-6$ & Trichloroethene & 0.50 & $\mathrm{U}$ \\
\hline $108-87-2$ & Methylcyclohexane & 0.50 & $\mathrm{U}$ \\
\hline $78-87-5$ & 1,2-Dichloropropane & 0.50 & $\mathrm{U}$ \\
\hline $75-27-4$ & Bromodichloromethane & 0.50 & $\mathrm{U}$ \\
\hline $10061-01-5$ & cis-1,3-Dichloropropene & 0.50 & $\mathrm{U}$ \\
\hline $108-10-1$ & 4-Methyl-2-pentanone & 5.0 & $\mathrm{U}$ \\
\hline $108-88-3$ & Toluene & 1.6 & \\
\hline $10061-02-6$ & trans-1,3-Dichloropropene & 0.50 & $\overline{\mathrm{U}}$ \\
\hline $79-00-5$ & 1, 1,2-Trichloroethane & 0.50 & $\mathrm{U}$ \\
\hline $127-18-4$ & Tetrachloroethene & 0.50 & $\mathrm{U}$ \\
\hline $591-78-6$ & 2-Hexanone & 5.0 & $\mathrm{U}$ \\
\hline $124-48-1$ & Dibromochloromethane & 0.50 & $\mathrm{U}$ \\
\hline $106-93-4$ & 1,2-Dibromoethane & 0.50 & $\mathrm{U}$ \\
\hline $108-90-7$ & Chlorobenzene & 0.50 & $\mathrm{U}$ \\
\hline $100-41-4$ & Ethylbenzene & 0.14 & $\mathrm{~J}$ \\
\hline $95-47-6$ & o-xylene & 0.50 & $\mathrm{U}$ \\
\hline $179601-23-1$ & $m, p-x y l e n e$ & 0.61 & \\
\hline $100-42-5$ & styrene & 0.50 & $\overline{\mathrm{U}}$ \\
\hline $75-25-2$ & Bromoform & 0.50 & $\mathrm{U}$ \\
\hline $98-82-8$ & Isopropylbenzene & 0.50 & $\mathrm{U}$ \\
\hline $79-34-5$ & $1,1,2,2$-Tetrachloroethane & 0.50 & $\mathrm{U}$ \\
\hline $541-73-1$ & 1,3-Dichlorobenzene & 0.50 & $\mathrm{U}$ \\
\hline $106-46-7$ & 1,4-Dichlorobenzene & 0.50 & $\mathrm{U}$ \\
\hline $95-50-1$ & 1,2-Dichlorobenzene & 0.50 & $\mathrm{U}$ \\
\hline $96-12-8$ & 1,2-Dibromo-3-chloropropane & 0.50 & $\mathrm{U}$ \\
\hline $120-82-1$ & $1,2,4$-Trichlorobenzene & 0.50 & $\mathrm{U}$ \\
\hline $87-61-6$ & $1,2,3$-Trichlorobenzene & 0.50 & $\mathrm{U}$ \\
\hline
\end{tabular}


1J - FORM I VOA-TIC

VOLATILE ORGANICS ANALYSIS DATA SHEET TENTATIVELY IDENTIFIED COMPOUNDS
EPA SAMPLE NO.

QCTBW2 7185
Lab Name: TESTAMERICA BURLINGTON

Lab Code: STLV Case No.: CNTRALIA

Matrix: (SOIL/SED/WATER) Water

Sample wt/vol: $25.0 \quad(\mathrm{~g} / \mathrm{mL}) \mathrm{mL}$

Level: (TRACE or LOW/MED) TRACE

\% Moisture: not dec.

GC Column: DB-624

ID $: 0.53$

Soil Extract Volume:

CONCENTRATION UNITS: (ug/L or $\mathrm{ug} / \mathrm{kg}) \mathrm{ug} / \mathrm{L}$
Contract: $8 \mathrm{E}-00302$

Mod. Ref No.:

SDG No.: 136697
Lab Sample ID: 825169

Lab File ID: 825169

Date Received: 04/07/2010

Date Analyzed: 04/08/2010

(mm) Dilution Factor: 1.0

(uL) Soil Aliquot Volume:

(uL)

Purge Volume: 25.0

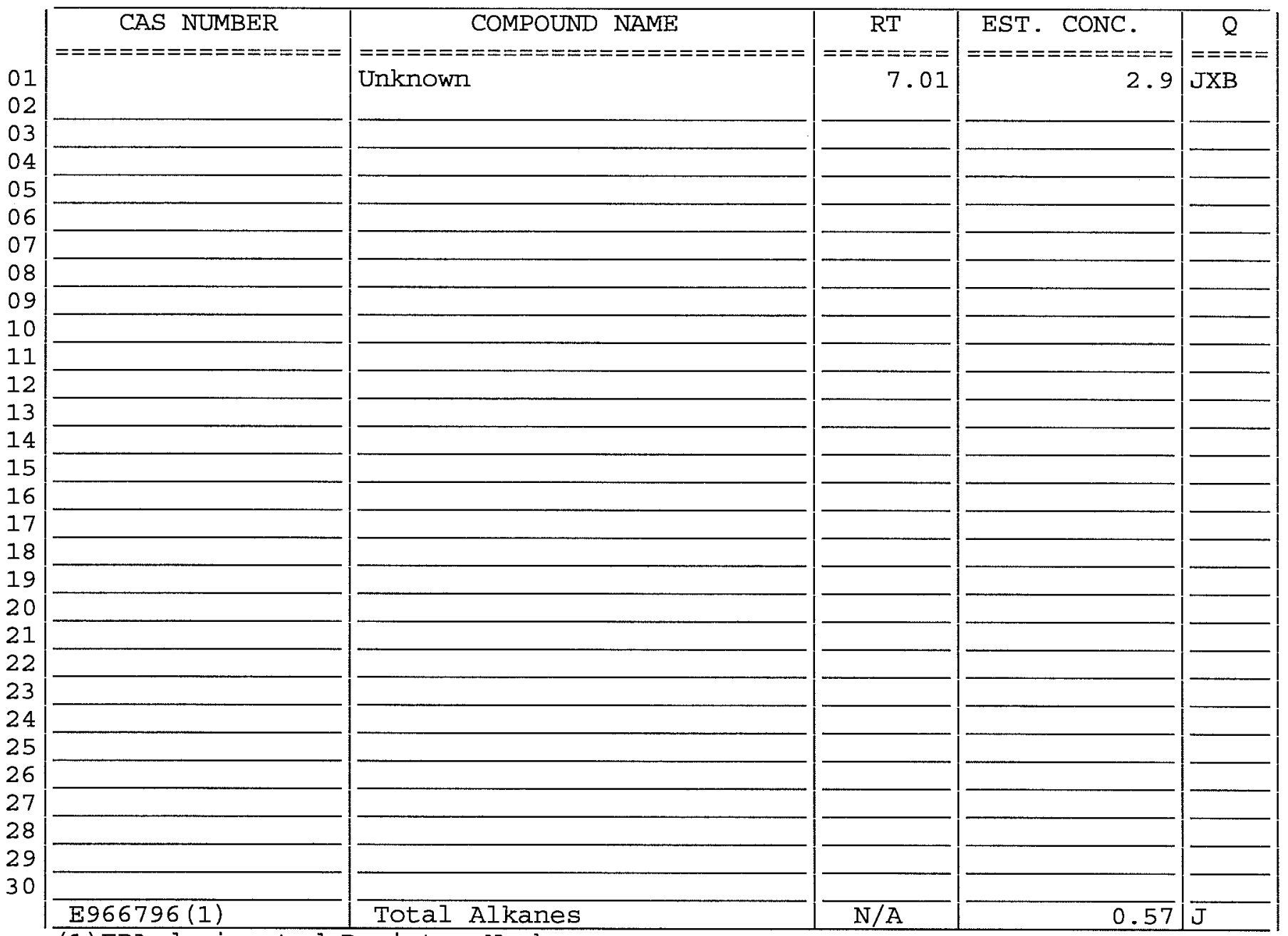

(1) EPA-designated Registry Number. 
$1 A$ - FORM I VOA-1

VOLATILE ORGANICS ANALYSIS DATA SHEET
EPA SAMPLE NO.

VBLTJM

Contract: $8 \mathrm{E}-00302$

Lab Code: STLV Case No.: CNTRALIA Mod. Ref No.:

SDG No.: 136697

Matrix: (SOIL/SED/WATER) Water

Sample wt/vol: $25.0 \quad(\mathrm{~g} / \mathrm{mL}) \mathrm{mL}$

Level: (TRACE/LOW/MED) TRACE

$\because$ Moisture: not dec.

GC Column: DB-624

Soil Extract Volume:

Purge Volume: 25.0
Lab Sample ID: VBLKJM

Lab File ID: JAQB03E

Date Received:

Date Analyzed: 04/08/2010

Dilution Factor: 1.0

Soil Aliquot Volume:

(uL)

\begin{tabular}{|c|c|c|c|}
\hline CAS NO. & COMPOUND & $\begin{array}{l}\text { CONCENTRATION UNITS: } \\
(\mathrm{ug} / \mathrm{L} \text { or } \mathrm{ug} / \mathrm{kg}) \mathrm{ug} / \mathrm{L}\end{array}$ & Q \\
\hline$===========0$ & 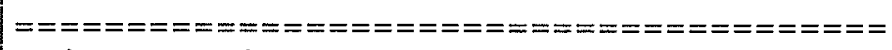 & 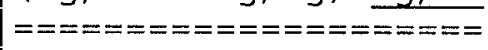 & $=======$ \\
\hline $75-71-8$ & Dichlorodifluoromethane & 0.50 & $\mathrm{U}$ \\
\hline $74-87-3$ & Chloromethane & 0.50 & $\mathrm{U}$ \\
\hline $75-01-4$ & Vinyl chloride & 0.50 & $\mathrm{U}$ \\
\hline $74-83-9$ & Bromomethane & 0.50 & $\mathrm{U}$ \\
\hline $75-00-3$ & Chloroethane & 0.50 & $\mathrm{U}$ \\
\hline $75-69-4$ & Trichlorofluoromethane & 0.50 & $\mathrm{U}$ \\
\hline $75-35-4$ & 1,1 -Dichloroethene & 0.50 & $\mathrm{U}$ \\
\hline $76-13-1$ & 1,1,2-Trichloro-1,2,2-trifluoroethane & 0.50 & $\mathrm{U}$ \\
\hline $67-64-1$ & Acetone & 3.0 & $\mathrm{~J}$ \\
\hline $75-15-0$ & Carbon disulfide & 0.50 & $\mathrm{U}$ \\
\hline $79-20-9$ & Methyl acetate & 0.50 & $\mathrm{U}$ \\
\hline $75-09-2$ & Methylene chloride & 0.50 & $\mathrm{U}$ \\
\hline $156-60-5$ & trans-1,2-Dichloroethene & 0.50 & $\mathrm{U}$ \\
\hline $1634-04-4$ & Methyl tert-butyl ether & 0.50 & $\mathrm{U}$ \\
\hline $75-34-3$ & 1,1-Dichloroethane & 0.50 & $\mathrm{U}$ \\
\hline $156-59-2$ & Cis-1,2-Dichloroethene & 0.50 & $\mathrm{U}$ \\
\hline $78-93-3$ & 2-Butanone & 1.2 & $\mathrm{~J}$ \\
\hline $74-97-5$ & Bromochloromethane & 0.50 & $\mathrm{U}$ \\
\hline $67-66-3$ & Chloroform & 0.50 & $\mathrm{U}$ \\
\hline $71-55-6$ & 1,1,1-Trichloroethane & 0.50 & $\mathrm{U}$ \\
\hline $110-82-7$ & Cyclohexane & 0.50 & $\mathrm{U}$ \\
\hline $56-23-5$ & Carbon tetrachloride & 0.50 & $\mathrm{U}$ \\
\hline $71-43-2$ & Benzene & 0.50 & $\mathrm{U}$ \\
\hline $107-06-2$ & 1,2-Dichloroethane & 0.50 & $\mathrm{U}$ \\
\hline
\end{tabular}

Report 1,4-Dioxane for Low-Medium VOA analysis only 
$1 B$ - FORM I VOA-2

VOLATILE ORGANICS ANALYSIS DATA SHEET
EPA SAMPLE NO.

VBLKJM
Lab Name: TESTAMERICA BURLINGTON

Case No.: CNTRALIA

Lab Code: STLV

Matrix: (SOIL/SED/WATER) Water

Sample wt/vol: 25.0

$(\mathrm{g} / \mathrm{mL}) \mathrm{mL}$

Level: (TRACE/LOW/MED) TRACE

\% Moisture: not dec.

GC Column: DB-624

ID $: 0.53$

Soil Extract Volume:

Purge Volume: 25.0
Contract: $8 \mathrm{E}-00302$

SDG No.: 136697
Lab Sample ID: VBLKJM

Lab File ID: JAQBO3E

Date Received:

Date Analyzed: 04/08/2010

Dilution Factor: 1.0

Soil Aliquot Volume:

\begin{tabular}{|c|c|c|c|}
\hline CAS NO. & COMPOUND & $\begin{array}{l}\text { CONCENTRATION UNITS: } \\
(u g / L \text { or } u g / \mathrm{kg}) \mathrm{ug} / \mathrm{L}\end{array}$ & $Q$ \\
\hline$======-=== \pm$ & 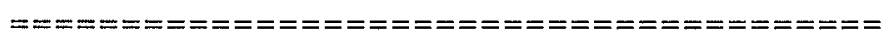 & 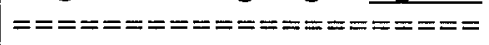 & $======$ \\
\hline $79-01-6$ & Trichloroethene & 0.50 & $\mathrm{U}$ \\
\hline $108-87-2$ & Methylcyclohexane & 0.50 & $\mathrm{U}$ \\
\hline $78-87-5$ & 1,2-Dichloropropane & 0.50 & $\mathrm{U}$ \\
\hline $75-27-4$ & Bromodichloromethane & 0.50 & $\mathrm{U}$ \\
\hline $10061-01-5$ & cis-1,3-Dichloropropene & 0.50 & $\mathrm{U}$ \\
\hline $108-10-1$ & 4-Methyl-2-pentanone & 5.0 & $\mathrm{U}$ \\
\hline $108-88-3$ & Toluene & 0.50 & $\mathrm{U}$ \\
\hline $10061-02-6$ & trans-1,3-Dichloropropene & 0.50 & $\mathrm{U}$ \\
\hline $79-00-5$ & 1,1,2-Trichloroethane & 0.50 & $\mathrm{U}$ \\
\hline $127-18-4$ & Tetrachloroethene & 0.50 & $\mathrm{U}$ \\
\hline $591-78-6$ & 2-Hexanone & 5.0 & $\mathrm{U}$ \\
\hline $124-48-1$ & Dibromochloromethane & 0.50 & $\mathrm{U}$ \\
\hline $106-93-4$ & 1,2-Dibromoethane & 0.50 & $\mathrm{U}$ \\
\hline $108-90-7$ & Chlorobenzene & 0.50 & $\mathrm{U}$ \\
\hline $100-41-4$ & Ethylbenzene & 0.50 & $\mathrm{U}$ \\
\hline $95-47-6$ & o-xylene & 0.50 & $\mathrm{U}$ \\
\hline $179601-23-1$ & $\mathrm{~m}, \mathrm{p}$-Xylene & 0.50 & $\mathrm{U}$ \\
\hline $100-42-5$ & Styrene & 0.50 & $\mathrm{U}$ \\
\hline $75-25-2$ & Bromoform & 0.50 & $\mathrm{U}$ \\
\hline $98-82-8$ & Isopropylbenzene & 0.50 & $\mathrm{U}$ \\
\hline $79-34-5$ & $1,1,2,2$-Tetrachloroethane & 0.50 & $\mathrm{U}$ \\
\hline $541-73-1$ & 1,3-Dichlorobenzene & 0.50 & $\mathrm{U}$ \\
\hline $106-46-7$ & 1,4-Dichlorobenzene & 0.50 & $\mathrm{U}$ \\
\hline $95-50-1$ & 1,2-Dichlorobenzene & 0.50 & $\mathrm{U}$ \\
\hline $96-12-8$ & 1,2-Dibromo-3-chloropropane & 0.50 & $\mathrm{U}$ \\
\hline $120-82-1$ & $1,2,4$-Trichlorobenzene & 0.50 & $\mathrm{U}$ \\
\hline $87-61-6$ & 1,2,3-Trichlorobenzene & 0.50 & $\mathrm{U}$ \\
\hline
\end{tabular}


IJ - FORM I VOA-TIC

VOLATILE ORGANICS ANALYSIS DATA SHEET

TENTATIVELY IDENTIFIED COMPOUNDS
EPA SAMPLE NO.

VBLKJM

Contract: $8 \mathrm{E}-00302$

Lab Name: TESTAMERICA BURLINGTON

Case No.: CNTRALIA

Mod. Ref No.:

SDG No.: 136697

Lab Sample ID: VBLKJM

Lab File ID: JAQB03E

Date Received:

Date Analyzed: 04/08/2010

GC Column: DB-624 ID: 0.53 (mm) Dilution Factor: 1.0

Soil Extract Volume:

(uL) Soil Aliquot Volume:

(uL)

CONCENTRATION UNITS: $(\mathrm{ug} / \mathrm{L}$ or $\mathrm{ug} / \mathrm{kg}) \mathrm{ug} / \mathrm{L}$ Purge Volume: 25.0

(mL)

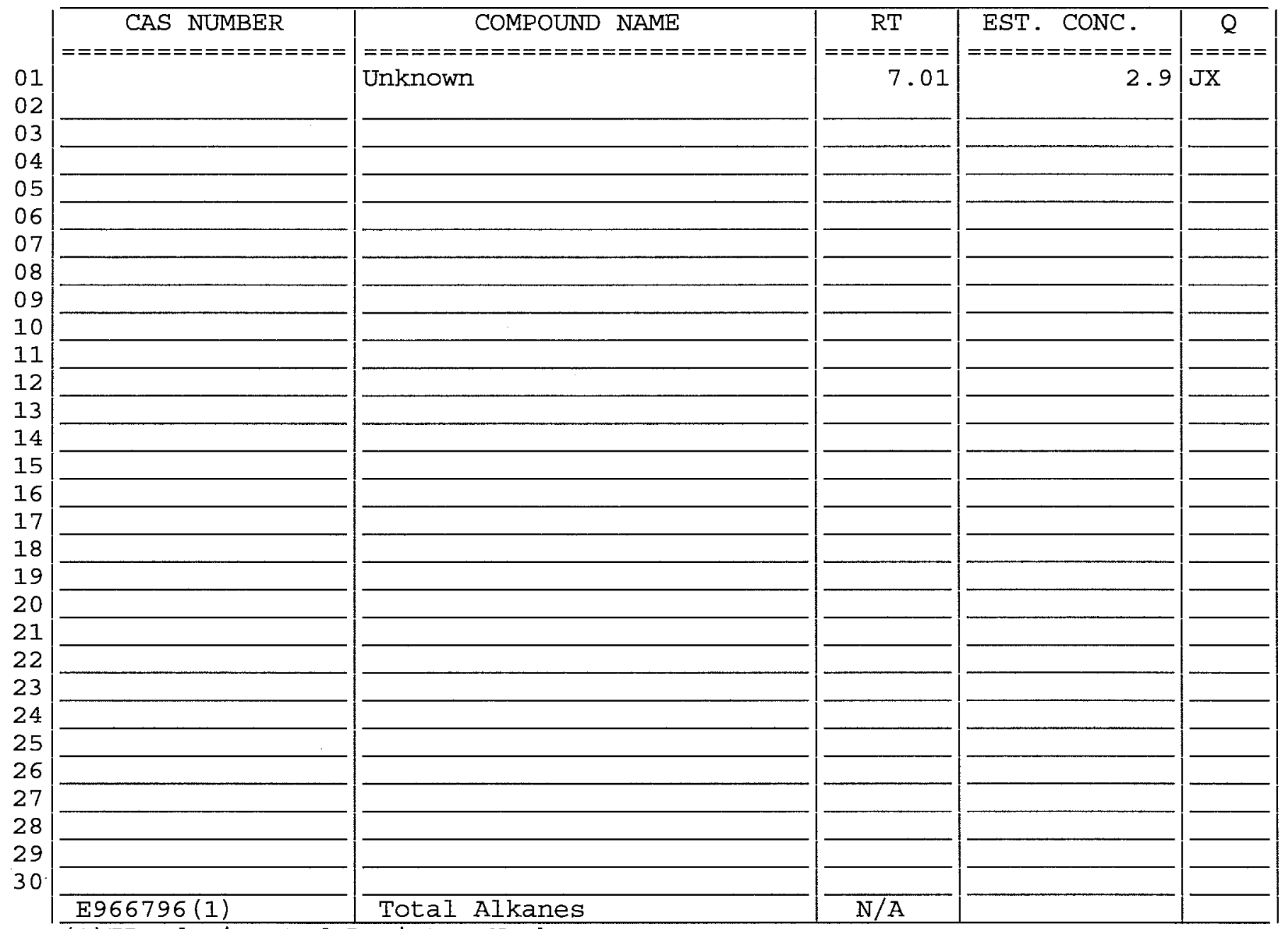

(1) EPA-designated Registry Number. 
$1 A$ - FORM I VOA-1

VOLATILE ORGANICS ANALYSIS DATA SHEET
EPA SAMPLE NO.

VBLKJN

Contract: $8 E-00302$
Lab Name: TESTAMERICA BURLINGTON

Lab Code: STLV Case No.: CNTRALIA Mod. Ref No.:
SDG NO.: 136697

Lab Sample ID: VBLKJN

Matrix: (SOIL/SED/WATER) Water

Sample wt/vol: $25.0 \quad(\mathrm{~g} / \mathrm{mL}) \mathrm{mL}$

Level: (TRACE/LOW/MED) TRACE

$\%$ Moisture: not dec.

GC Column: DB-624

Soil Extract Volume:

ID: 0.53

(mm)

(uL)

(mL)

Purge Volume: 25.0
Lab File ID: JAQBO2F

Date Received:

Date Analyzed: 04/09/2010

Dilution Factor: 1.0

Soil Aliquot Volume:

\begin{tabular}{|c|c|c|c|}
\hline CAS NO. & COMPOUND & $\begin{array}{l}\text { CONCENTRATION UNITS: } \\
(\mathrm{ug} / \mathrm{L} \text { or } \mathrm{ug} / \mathrm{kg}) \mathrm{ug} / \mathrm{L}\end{array}$ & $\mathrm{Q}$ \\
\hline$=========$ & 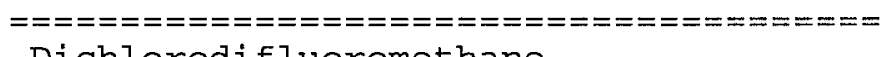 & $================$ & $======$ \\
\hline $75-71-8$ & Dichlorodifluoromethane & 0.50 & $\mathrm{U}$ \\
\hline $74-87-3$ & Chloromethane & 0.50 & $\mathrm{U}$ \\
\hline $75-01-4$ & Vinyl chloride & 0.50 & $\mathrm{U}$ \\
\hline $74-83-9$ & Bromomethane & 0.50 & $\mathrm{U}$ \\
\hline $75-00-3$ & Chloroethane & 0.50 & $\mathrm{U}$ \\
\hline $75-69-4$ & Trichlorofluoromethane & 0.50 & $\mathrm{U}$ \\
\hline $75-35-4$ & 1,1-Dichloroethene & 0.50 & $\mathrm{U}$ \\
\hline $76-13-1$ & $1,1,2$-Trichloro-1,2,2-trifluoroethane & 0.50 & $\mathrm{U}$ \\
\hline $67-64-1$ & Acetone & 2.6 & $\mathrm{~J}$ \\
\hline $75-15-0$ & Carbon disulfide & 0.50 & $\mathrm{U}$ \\
\hline $79-20-9$ & Methyl acetate & 0.50 & $\mathrm{U}$ \\
\hline $75-09-2$ & Methylene chloride & 0.50 & $\mathrm{U}$ \\
\hline $156-60-5$ & trans-1,2-Dichloroethene & 0.50 & U \\
\hline $1634-04-4$ & Methyl tert-butyl ether & 0.50 & $\mathrm{U}$ \\
\hline $75-34-3$ & 1,1-Dichloroethane & 0.50 & $\mathrm{U}$ \\
\hline $156-59-2$ & cis-1,2-Dichloroethene & 0.50 & $\mathrm{U}$ \\
\hline $78-93-3$ & 2-Butanone & 1.1 & $\mathrm{~J}$ \\
\hline $74-97-5$ & Bromochloromethane & 0.50 & U \\
\hline $67-66-3$ & Chloroform & 0.50 & $\mathrm{U}$ \\
\hline $71-55-6$ & 1,1,1-Trichloroethane & 0.50 & $\mathrm{U}$ \\
\hline $110-82-7$ & Cyclohexane & 0.50 & $\mathrm{U}$ \\
\hline $56-23-5$ & Carbon tetrachloride & 0.50 & $\mathrm{U}$ \\
\hline $71-43-2$ & Benzene & 0.50 & $\mathrm{U}$ \\
\hline $107-06-2$ & 1,2-Dichloroethane & 0.50 & $\mathrm{U}$ \\
\hline
\end{tabular}

Report 1,4-Dioxane for Low-Medium VOA analysis only 
$1 B$ - FORM I VOA-2

VOLATILE ORGANICS ANALYSIS DATA SHEET
EPA SAMPLE NO.

VBLKJN

Lab Name: TESTAMERICA BURLINGTON

Contract : $8 \mathrm{E}-00302$

Lab Code: STLV Case No.: CNTRALIA Mod. Ref No.:

SDG No. : 136697

Matrix: (SOIL/SED/WATER) water

Sample wt/vol: 25.0

$(\mathrm{g} / \mathrm{mL}) \mathrm{mL}$

Level: (TRACE/LOW/MED) TRACE

\% Moisture: not dec.

GC Column: DB-624

ID $: 0.53$

Soil Extract Volume:

Purge Volume: 25.0
Lab Sample ID: VBLKJN

Lab File ID: JAQBO2F

Date Received:

Date Analyzed: 04/09/2010

Dilution Factor: 1.0

Soil Aliquot Volume:

(uL)

\begin{tabular}{|c|c|c|c|}
\hline CAS NO. & COMPOUND & $\begin{array}{l}\text { CONCENTRATION UNITS: } \\
(\mathrm{ug} / \mathrm{L} \text { or } u g / \mathrm{kg} \text { ) ug/L }\end{array}$ & $Q$ \\
\hline$===========$ & 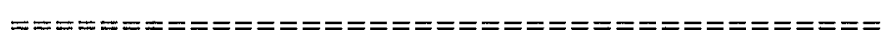 & 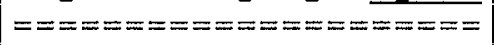 & $=======$ \\
\hline $79-01-6$ & Trichloroethene & 0.50 & $\mathrm{U}$ \\
\hline $108-87-2$ & Methylcyclohexane & 0.50 & $\mathrm{U}$ \\
\hline $78-87-5$ & 1,2-Dichloropropane & 0.50 & $\mathrm{U}$ \\
\hline $75-27-4$ & Bromodichloromethane & 0.50 & $\mathrm{U}$ \\
\hline $10061-01-5$ & cis-1,3-Dichloropropene & 0.50 & $\mathrm{U}$ \\
\hline $108-10-1$ & 4-Methyl-2-pentanone & 5.0 & $\mathrm{U}$ \\
\hline $108-88-3$ & Toluene & 0.50 & $\mathrm{U}$ \\
\hline $10061-02-6$ & trans-1,3-Dichloropropene & 0.50 & $\mathrm{U}$ \\
\hline $79-00-5$ & $1,1,2$-Trichloroethane & 0.50 & $\mathrm{U}$ \\
\hline $127-18-4$ & Tetrachloroethene & 0.50 & $\mathrm{U}$ \\
\hline $591-78-6$ & 2-Hexanone & 5.0 & $\mathrm{U}$ \\
\hline $124-48-1$ & Dibromochloromethane & 0.50 & $\mathrm{U}$ \\
\hline $106-93-4$ & 1,2-Dibromoethane & 0.50 & $\mathrm{U}$ \\
\hline $108-90-7$ & Chlorobenzene & 0.50 & $\mathrm{U}$ \\
\hline $100-41-4$ & Ethylbenzene & 0.50 & $\mathrm{U}$ \\
\hline $95-47-6$ & o-xylene & 0.50 & $\mathrm{U}$ \\
\hline $179601-23-1$ & m, p-xylene & 0.50 & $\mathrm{U}$ \\
\hline $100-42-5$ & styrene & 0.50 & $\mathrm{U}$ \\
\hline $75-25-2$ & Bromoform & 0.50 & $\mathrm{U}$ \\
\hline $98-82-8$ & Isopropylbenzene & 0.50 & $\mathrm{U}$ \\
\hline $79-34-5$ & $1,1,2,2$-Tetrachloroethane & 0.50 & $\mathrm{U}$ \\
\hline $541-73-1$ & 1,3-Dichlorobenzene & 0.50 & $\mathrm{U}$ \\
\hline $106-46-7$ & 1,4-Dichlorobenzene & 0.50 & $\mathrm{U}$ \\
\hline $95-50-1$ & 1,2-Dichlorobenzene & 0.50 & $\mathrm{U}$ \\
\hline $96-12-8$ & 1,2-Dibromo-3-chloropropane & 0.50 & $\mathrm{U}$ \\
\hline $120-82-1$ & $1,2,4-$ Trichlorobenzene & 0.50 & $\mathrm{U}$ \\
\hline $87-61-6$ & $1,2,3$-Trichlorobenzene & 0.50 & $\mathrm{U}$ \\
\hline
\end{tabular}


IJ - FORM I VOA-TIC

VOLATILE ORGANICS ANALYSIS DATA SHEET TENTATIVELY IDENTIFIED COMPOUNDS
EPA SAMPLE NO.

VBLKJN

Contract: $8 \mathrm{E}-00302$

Lab Name: TESTAMERICA BURLINGTON

Mod. Ref No.:

SDG No.: 136697

Lab Sample ID: VBLKJN

Lab File ID: JAQBO2F

Date Received:

Date Analyzed: 04/09/2010

\% Moisture: not dec.

(mm) Dilution Factor: 1.0

GC Column: DB-624

ID $: 0.53$

(uL) Soil Aliquot Volume:

(uL)

Soil Extract Volume:

Purge Volume: 25.0

(mL)

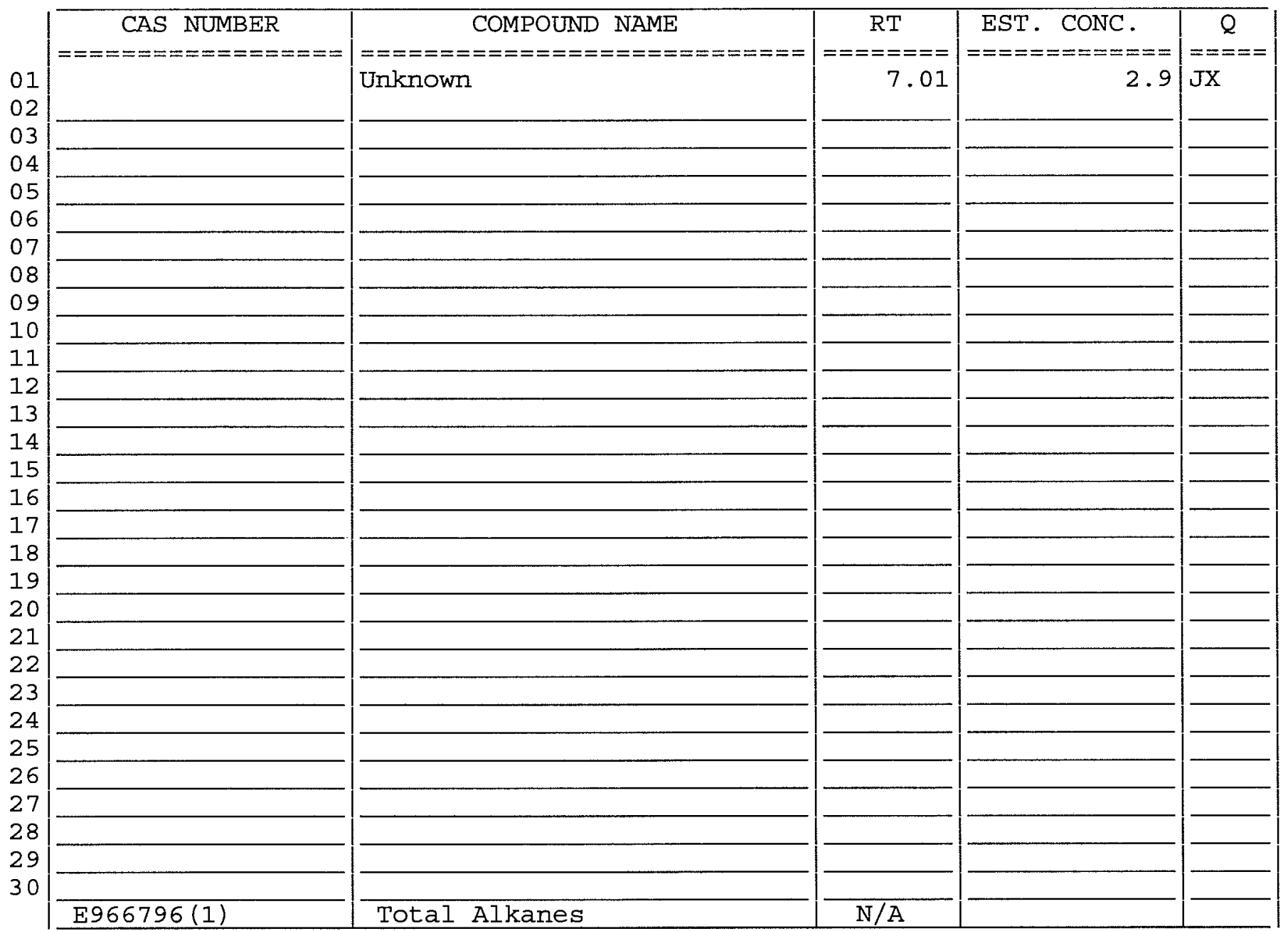

(1) EPA-designated Registry Number. 
$1 A$ - FORM I VOA-1

VOLATILE ORGANICS ANALYSIS DATA SHEET
EPA SAMPLE NO.

VBLKJO

Lab Name: TESTAMERICA BURLINGTON

Contract: $8 E-00302$

Lab Code: STLV Case No.: CNTRALIA Mod. Ref No.:

SDG No.: 136697

Matrix: (SOIL/SED/WATER) water

Sample wt/vol: 25.0

$(\mathrm{g} / \mathrm{mL}) \mathrm{mL}$

Level: (TRACE/LOW/MED) TRACE

\% Moisture: not dec.

GC Column: DB-624

ID : 0.53

Soil Extract Volume:

Purge Volume: 25.0
Lab Sample ID: VBLKJO

Lab File ID: JAQBO2G

Date Received:

Date Analyzed: 04/10/2010

Dilution Factor: 1.0

Soil Aliquot Volume:

(uL)

\begin{tabular}{|c|c|c|c|}
\hline CAS NO. & COMPOUND & $\begin{array}{l}\text { CONCENTRATION UNITS: } \\
(u g / L \text { or } u g / k g) u g / L\end{array}$ & $Q$ \\
\hline$====-====$ & 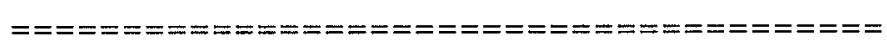 & 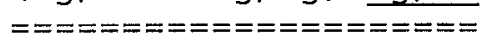 & $=======$ \\
\hline $75-71-8$ & Dichlorodifluoromethane & 0.50 & $\mathrm{U}$ \\
\hline $74-87-3$ & Chloromethane & 0.50 & $\mathrm{U}$ \\
\hline $75-01-4$ & Vinyl chloride & 0.50 & $\mathrm{U}$ \\
\hline $74-83-9$ & Bromomethane & 0.50 & $\mathrm{U}$ \\
\hline $75-00-3$ & Chloroethane & 0.50 & $\mathrm{U}$ \\
\hline $75-69-4$ & Trichlorofluoromethane & 0.50 & $\mathrm{U}$ \\
\hline $75-35-4$ & 1,1-Dichloroethene & 0.50 & $\mathrm{U}$ \\
\hline $76-13-1$ & 1,1,2-Trichloro-1,2,2-trifluoroethane & 0.50 & $\mathrm{U}$ \\
\hline $67-64-1$ & Acetone & 2.6 & $\mathrm{~J}$ \\
\hline $75-15-0$ & Carbon disulfide & 0.50 & $\mathrm{U}$ \\
\hline $79-20-9$ & Methyl acetate & 0.50 & $\mathrm{U}$ \\
\hline $75-09-2$ & Methylene chloride & 0.50 & $\mathrm{U}$ \\
\hline $156-60-5$ & trans-1,2-Dichloroethene & 0.50 & $\mathrm{U}$ \\
\hline $1634-04-4$ & Methyl tert-butyl ether & 0.50 & $\mathrm{U}$ \\
\hline $75-34-3$ & 1,1 -Dichloroethane & 0.50 & $\mathrm{U}$ \\
\hline $156-59-2$ & cis-1,2-Dichloroethene & 0.50 & $\mathrm{U}$ \\
\hline $78-93-3$ & 2-Butanone & 1.3 & $\mathrm{~J}$ \\
\hline $74-97-5$ & Bromochloromethane & 0.50 & $\mathrm{U}$ \\
\hline $67-66-3$ & Chloroform & 0.50 & $\mathrm{U}$ \\
\hline $71-55-6$ & $1,1,1$-Trichloroethane & 0.50 & $\mathrm{U}$ \\
\hline $110-82-7$ & Cyclohexane & 0.50 & $\mathrm{U}$ \\
\hline $56-23-5$ & Carbon tetrachloride & 0.50 & $\mathrm{U}$ \\
\hline $71-43-2$ & Benzene & 0.50 & $\mathrm{U}$ \\
\hline $107-06-2$ & 1,2-Dichloroethane & 0.50 & $\mathrm{U}$ \\
\hline
\end{tabular}

Report 1,4-Dioxane for Low-Medium VOA analysis only 
IB - FORM I VOA-2

VOLATILE ORGANICS ANALYSIS DATA SHEET
EPA SAMPLE NO.

VBLKJO
Lab Name: TESTAMERICA BURLINGTON

Case No.: CNTRALIA

Mod. Ref No.:
Contract : $8 E-00302$

SDG No.: 136697
Matrix: (SOIL/SED/WATER) water

Sample wt/vol: 25.0

$(\mathrm{g} / \mathrm{mL}) \mathrm{mL}$

Level: (TRACE/LOW/MED) TRACE

\% Moisture: not dec.

GC Column: DB-624

Soil Extract Volume:

ID $: 0.53$

$(\mathrm{mm})$

(uL)

(mL)

Purge Volume: 25.0
Lab Sample ID: VBLKJO

Lab File ID: JAQB02G

Date Received:

Date Analyzed: 04/10/2010

Dilution Factor: 1.0

Soil Aliquot Volume:

(uL)

\begin{tabular}{|c|c|c|c|}
\hline CAS NO. & COMPOUND & $\begin{array}{l}\text { CONCENTRATION UNITS: } \\
\text { (ug/L or ug/kg) ug/L }\end{array}$ & Q \\
\hline$==========$ & 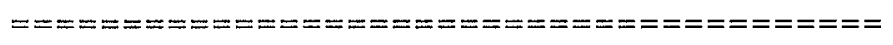 & 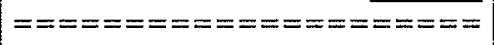 & $======-$ \\
\hline $79-01-6$ & Trichloroethene & 0.50 & $\mathrm{U}$ \\
\hline $108-87-2$ & Methylcyclohexane & 0.50 & $\mathrm{U}$ \\
\hline $78-87-5$ & 1,2-Dichloropropane & 0.50 & $\mathrm{U}$ \\
\hline $75-27-4$ & Bromodichloromethane & 0.50 & $\mathrm{U}$ \\
\hline $10061-01-5$ & cis-1,3-Dichloropropene & 0.50 & $\mathrm{U}$ \\
\hline $108-10-1$ & 4-Methyl-2-pentanone & 5.0 & $\mathrm{U}$ \\
\hline $108-88-3$ & Toluene & 0.50 & $\mathrm{U}$ \\
\hline $10061-02-6$ & trans-1,3-Dichloropropene & 0.50 & $\mathrm{U}$ \\
\hline $79-00-5$ & $1,1,2$-Trichloroethane & 0.50 & $\mathrm{U}$ \\
\hline $127-18-4$ & Tetrachloroethene & 0.50 & $\mathrm{U}$ \\
\hline $591-78-6$ & 2-Hexanone & 5.0 & $\mathrm{U}$ \\
\hline $124-48-1$ & Dibromochloromethane & 0.50 & $\mathrm{U}$ \\
\hline $106-93-4$ & I,2-Dibromoethane & 0.50 & $\mathrm{U}$ \\
\hline $108-90-7$ & Chlorobenzene & 0.50 & $\mathrm{U}$ \\
\hline $100-41-4$ & Ethylbenzene & 0.50 & $\mathrm{U}$ \\
\hline $95-47-6$ & o-xylene & 0.50 & $\mathrm{U}$ \\
\hline $179601-23-1$ & $\mathrm{~m}, \mathrm{p}$-Xylene & 0.50 & $\mathrm{U}$ \\
\hline $100-42-5$ & styrene & 0.50 & $\mathrm{U}$ \\
\hline $75-25-2$ & Bromoform & 0.50 & $\mathrm{U}$ \\
\hline $98-82-8$ & Isopropylbenzene & 0.50 & $\mathrm{U}$ \\
\hline $79-34-5$ & $1,1,2,2$-Tetrachloroethane & 0.50 & $\mathrm{U}$ \\
\hline $541-73-1$ & 1,3-Dichlorobenzene & 0.50 & $\mathrm{U}$ \\
\hline $106-46-7$ & 1,4-Dichlorobenzene & 0.50 & $\mathrm{U}$ \\
\hline $95-50-1$ & 1,2-Dichlorobenzene & 0.50 & $\mathrm{U}$ \\
\hline $96-12-8$ & 1,2-Dibromo-3-chloropropane & 0.50 & $\mathrm{U}$ \\
\hline $120-82-1$ & 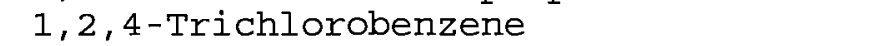 & 0.50 & $\mathrm{U}$ \\
\hline $87-61-6$ & 1,2,3-Trichlorobenzene & 0.50 & $\mathrm{U}$ \\
\hline
\end{tabular}


IJ - FORM I VOA-TIC

VOLATILE ORGANICS ANALYSIS DATA SHEET

TENTATIVELY IDENTIFIED COMPOUNDS
EPA SAMPLE NO.

VBLKJO
Lab Name: TESTAMERICA BURLINGTON

Lab Code: STLV Case No.: CNTRALIA

Matrix: (SOIL/SED/WATER) Water

Sample wt/vol: $25.0 \quad(\mathrm{~g} / \mathrm{mL}) \mathrm{mL}$

Level: (TRACE or LOW/MED) TRACE

\% Moisture: not dec.

GC Column: DB-624

ID $: 0.53$

Soil Extract Volume:

CONCENTRATION UNITS: (ug/L or $\mathrm{ug} / \mathrm{kg}) \mathrm{ug} / \mathrm{L}$
Contract: $8 \mathrm{E}-00302$

Mod. Ref No.:

SDG No.: 136697
Lab Sample ID: VBLKJO

Lab File ID: JAQB02G

Date Received:

Date Analyzed: 04/10/2010

(mm) Dilution Factor: 1.0

(uL) Soil Aliquot Volume:

(uL)

Purge Volume: 25.0

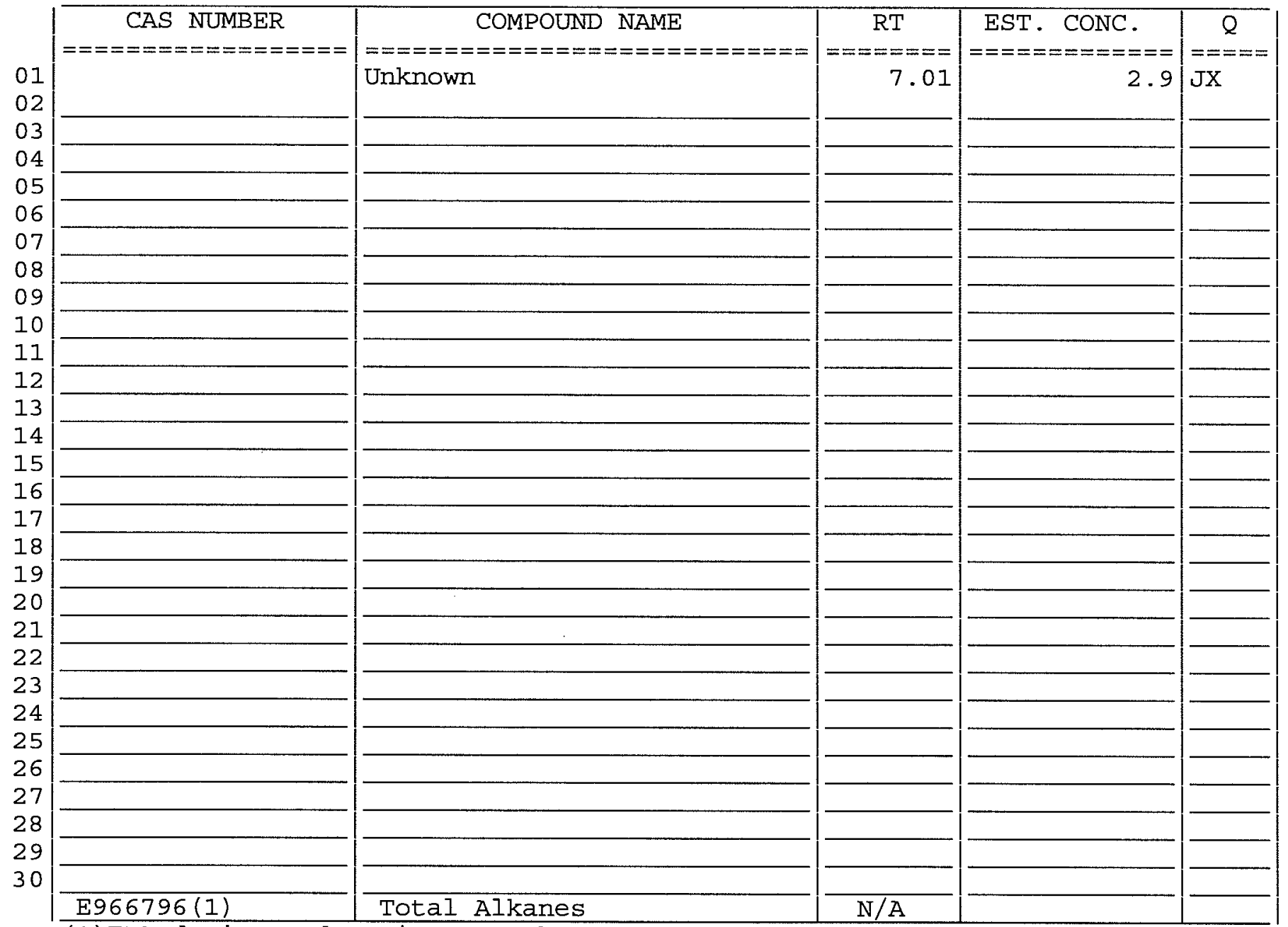

(1) EPA-designated Registry Number. 
$1 A$ - FORM I VOA-1

VOLATILE ORGANICS ANALYSIS DATA SHEET
EPA SAMPLE NO.

VHBLK01
Lab Name: TESTAMERICA BURLINGTON

Lab Code: STLV

Case No.: CNTRALIA

Mod

Matrix: (SOIL/SED/WATER) Water

Sample wt/vol: 25.0

$(\mathrm{g} / \mathrm{mL}) \mathrm{mL}$

Leve1: (TRACE/LOW/MED) TRACE

\% Moisture: not dec.

GC Column: DB-624

ID : 0.53

Soil Extract Volume:

Purge Volume: 25.0
Contract: 8E-00302

SDG No.: 136697
Lab Sample ID: 825170

Lab File ID: 825170

Date Received:

Date Analyzed: 04/10/2010

Dilution Factor: 1.0

Soil Aliquot Volume:

(uL)

\begin{tabular}{|c|c|c|c|}
\hline CAS NO. & COMPOUND & $\begin{array}{l}\text { CONCENTRATION UNITS: } \\
(\mathrm{ug} / \mathrm{L} \text { or } \mathrm{ug} / \mathrm{kg}) \mathrm{ug} / \mathrm{L}\end{array}$ & Q \\
\hline 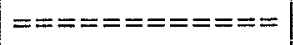 & 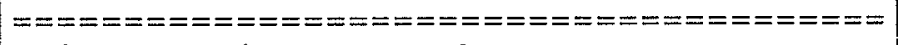 & 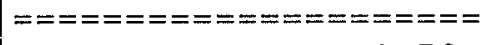 & 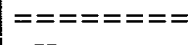 \\
\hline $75-71-8$ & Dichlorodifluoromethane & 0.50 & $\mathrm{U}$ \\
\hline $74-87-3$ & Chloromethane & 0.50 & $\mathrm{U}$ \\
\hline $75-01-4$ & Vinyl chloride & 0.50 & $\mathrm{U}$ \\
\hline $74-83-9$ & Bromomethane & 0.50 & $\mathrm{U}$ \\
\hline $75-00-3$ & Chloroethane & 0.50 & $\mathrm{U}$ \\
\hline $75-69-4$ & Trichlorofluoromethane & 0.50 & $\mathrm{U}$ \\
\hline $75-35-4$ & 1,1-Dichloroethene & 0.50 & $\mathrm{U}$ \\
\hline $76-13-1$ & 1,1,2-Trichloro-1,2,2-trifluoroethane & 0.50 & $\mathrm{U}$ \\
\hline $67-64-1$ & Acetone & 1.3 & JB \\
\hline $75-15-0$ & Carbon disulfide & 0.50 & $\mathrm{U}$ \\
\hline $79-20-9$ & Methyl acetate & 0.50 & $\mathrm{U}$ \\
\hline $75-09-2$ & Methylene chloride & 0.50 & $\mathrm{U}$ \\
\hline $156-60-5$ & trans-1,2-Dichloroethene & 0.50 & $\mathrm{U}$ \\
\hline $1634-04-4$ & Methyl tert-butyl ether & 0.50 & $\mathrm{U}$ \\
\hline $75-34-3$ & 1,1-Dichloroethane & 0.50 & $\mathrm{U}$ \\
\hline $156-59-2$ & cis-1,2-Dichloroethene & 0.50 & U \\
\hline $78-93-3$ & 2-Butanone & 0.46 & JB \\
\hline $74-97-5$ & Bromochloromethane & 0.50 & $\mathrm{U}$ \\
\hline $67-66-3$ & Chloroform & 0.36 & J \\
\hline $71-55-6$ & 1,1,1-Trichloroethane & 0.50 & $\mathrm{U}$ \\
\hline $110-82-7$ & Cyclohexane & 0.50 & $\mathrm{U}$ \\
\hline $56-23-5$ & Carbon tetrachloride & 0.50 & $\mathrm{U}$ \\
\hline $71-43-2$ & Benzene & 0.50 & $\mathrm{U}$ \\
\hline $107-06-2$ & 1,2-Dichloroethane & 0.50 & $\mathrm{U}$ \\
\hline
\end{tabular}

Report 1,4-Dioxane for Low-Medium VOA analysis only 
Lab Name: TESTAMERICA BURLINGTON

Contract: $8 \mathrm{E}-00302$

Lab Code: STLV Case No.: CNTRALIA Mod. Ref No.:

SDG No.: 136697

Matrix: (SOIL/SED/WATER) Water

Sample wt/vol: 25.0

$(\mathrm{g} / \mathrm{mL}) \mathrm{mL}$

Level: (TRACE/LOW/MED) TRACE

\% Moisture: not dec.

GC Column: DB-624

ID $: 0.53$

Soil Extract Volume:

Purge Volume: 25.0
Lab Sample ID: 825170

Lab File ID: 825170

Date Received:

Date Analyzed: 04/10/2010

Dilution Factor: 1.0

Soil Aliquot Volume:

(uL)

\begin{tabular}{|c|c|c|c|}
\hline CAS NO. & COMPOUND & $\begin{array}{l}\text { CONCENTRATION UNITS: } \\
(\mathrm{ug} / \mathrm{L} \text { or } \mathrm{ug} / \mathrm{kg}) \mathrm{ug} / \mathrm{L} \\
\end{array}$ & Q \\
\hline$==========$ & 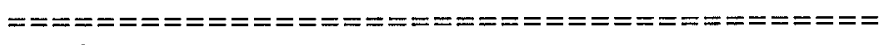 & 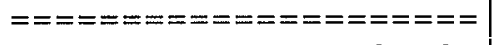 & 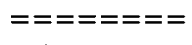 \\
\hline $79-0 I-6$ & Trichloroethene & $\quad 0.50$ & $\mathrm{U}$ \\
\hline $108-87-2$ & Methylcyclohexane & 0.50 & $\mathrm{U}$ \\
\hline $78-87-5$ & 1,2-Dichloropropane & 0.50 & $\mathrm{U}$ \\
\hline $75-27-4$ & Bromodichloromethane & 0.50 & $\mathrm{U}$ \\
\hline $10061-01-5$ & cis-1,3-Dichloropropene & 0.50 & $\mathrm{U}$ \\
\hline $108-10-1$ & 4-Methyl-2-pentanone & 5.0 & $\mathrm{U}$ \\
\hline $108-88-3$ & Toluene & 0.50 & $\mathrm{U}$ \\
\hline $10061-02-6$ & trans-1,3-Dichloropropene & 0.50 & $\mathrm{U}$ \\
\hline $79-00-5$ & 1,1,2-Trichloroethane & 0.50 & $\mathrm{U}$ \\
\hline $127-18-4$ & Tetrachloroethene & 0.50 & $\mathrm{U}$ \\
\hline $591-78-6$ & 2-Hexanone & 5.0 & $\mathrm{U}$ \\
\hline $124-48-I$ & Dibromochloromethane & 0.50 & $\mathrm{U}$ \\
\hline $106-93-4$ & 1,2-Dibromoethane & 0.50 & $\mathrm{U}$ \\
\hline $108-90-7$ & Chlorobenzene & 0.50 & $\mathrm{U}$ \\
\hline $100-41-4$ & Ethylbenzene & 0.50 & $\mathrm{U}$ \\
\hline $95-47-6$ & o-Xylene & 0.50 & $\mathrm{U}$ \\
\hline $179601-23-1$ & $\mathrm{~m}, \mathrm{p}$-Xylene & 0.50 & $\mathrm{U}$ \\
\hline $100-42-5$ & styrene & 0.50 & $\mathrm{U}$ \\
\hline $75-25-2$ & Bromoform & 0.50 & $\mathrm{U}$ \\
\hline $98-82-8$ & Isopropylbenzene & 0.50 & $\mathrm{U}$ \\
\hline $79-34-5$ & $1,1,2,2$-Tetrachloroethane & 0.50 & $\mathrm{U}$ \\
\hline $541-73-1$ & 1,3-Dichlorobenzene & 0.50 & $\mathrm{U}$ \\
\hline $106-46-7$ & 1,4-Dichlorobenzene & 0.50 & $\mathrm{U}$ \\
\hline $95-50-1$ & 1,2-Dichlorobenzene & 0.50 & $\mathrm{U}$ \\
\hline $96-12-8$ & 1,2-Dibromo-3-chloropropane & 0.50 & $\mathrm{U}$ \\
\hline $120-82-1$ & $1,2,4$-Trichlorobenzene & 0.50 & $\mathrm{U}$ \\
\hline $87-6 I-6$ & $1,2,3$-Trichlorobenzene & 0.50 & $\mathrm{U}$ \\
\hline
\end{tabular}


IJ - FORM I VOA-TIC

VOIATILE ORGANICS ANALYSIS DATA SHEET

TENTATIVELY IDENTIFIED COMPOUNDS
EPA SAMPLE NO.

VHBLKO 1
Lab Name: TESTAMERICA BURLINGTON

Lab Code: STLV Case No.: CNTRALIA

Matrix: (SOIL/SED/WATER) Water

Sample wt/vol: $25.0 \quad(\mathrm{~g} / \mathrm{mL}) \mathrm{mL}$

Level: (TRACE or LOW/MED) TRACE

\% Moisture: not dec.

GC Column: DB-624

ID $: 0.53$

Soil Extract Volume:

CONCENTRATION UNITS: (ug/L or ug/kg) ug/L
Contract: 8E-00302

Mod. Ref No.:

SDG NO. : 136697

Lab File ID: 825170

Date Received:

Date Analyzed: 04/10/2010

(mm) Dilution Factor: 1.0

(uL) Soil Aliquot Volume:

(uL)

Purge Volume: 25.0

(mL)

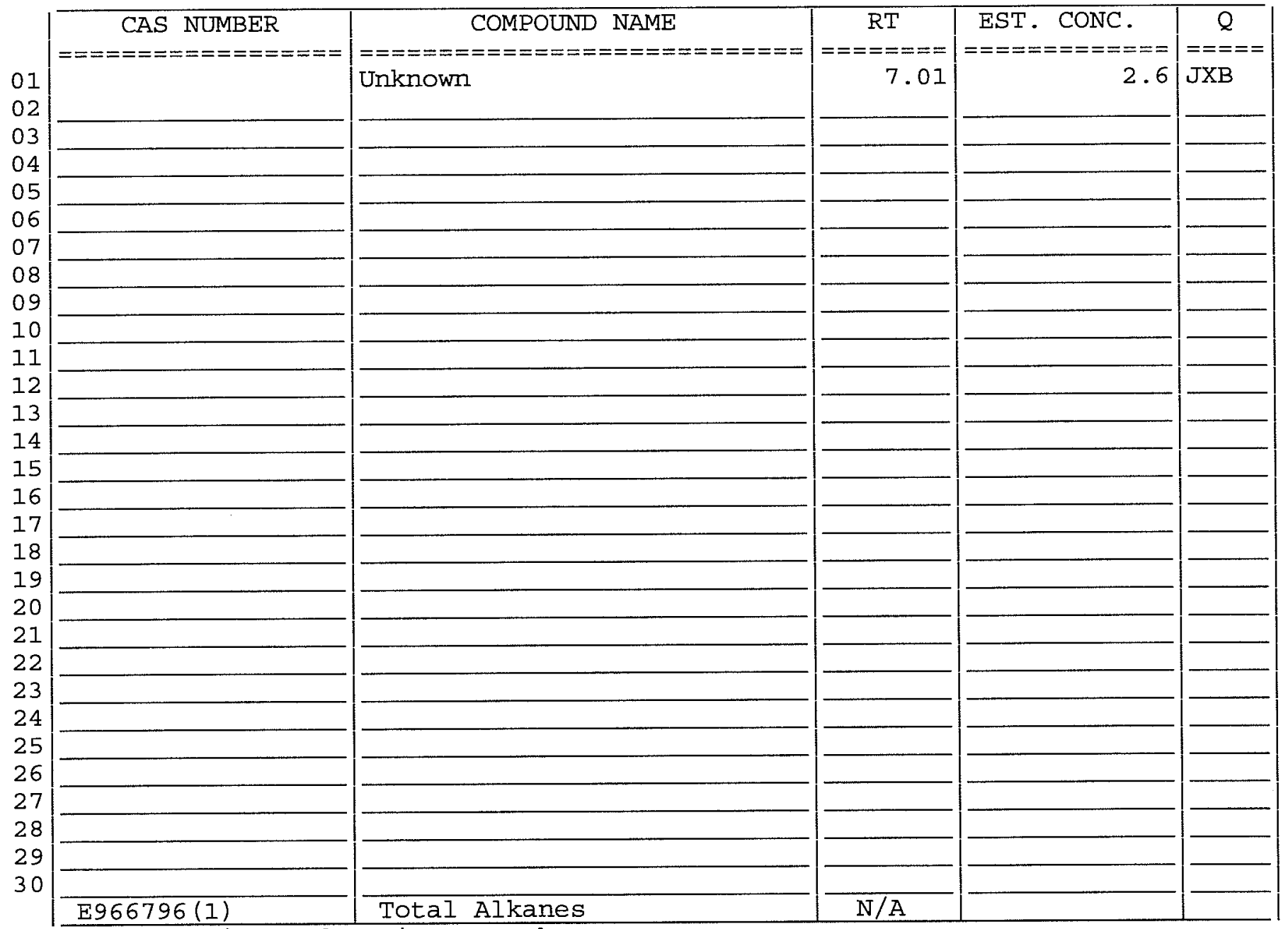

(1) EPA-designated Registry Number. 
$2 A$ - FORM II VOA-1

WATER VOLATILE DEUTERATED MONITORING COMPOUND RECOVERY

Lab Name: TESTAMERICA BURLINGTON

Contract: $8 \mathrm{E}-00302$

Lab Code: STLV Case No.: CNTRALIA Mod. Ref No.:

SDG No.: 136697

Level: (TRACE or LOW) TRACE

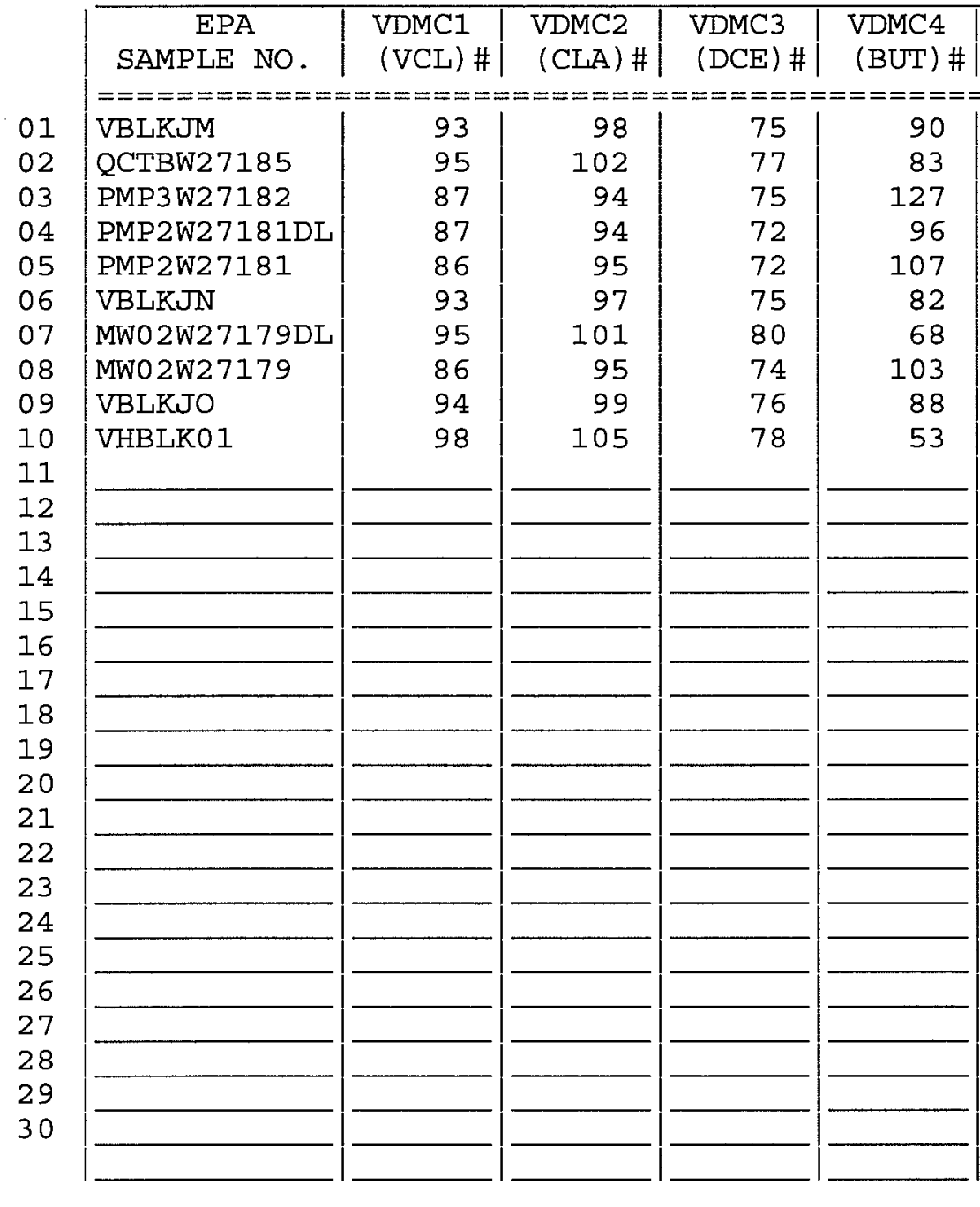
VDMC1 (VCL) = Vinyl chloride $-\mathrm{d} 3$
VDMC2 $(C L A)=$ Chloroethane $-d 5$
VDMC3 $(\mathrm{DCE})=1,1-\mathrm{Dich}$ loroethene-d2
VDMC4 $(\mathrm{BUT})=2$-Butanone-d5
VDMC5 $(\mathrm{CLF})=$ Chloroform-d
VDMC6 $(D C A)=1,2-$ Dichloroethane-d4
VDMC7 (BEN) = Benzene-d6

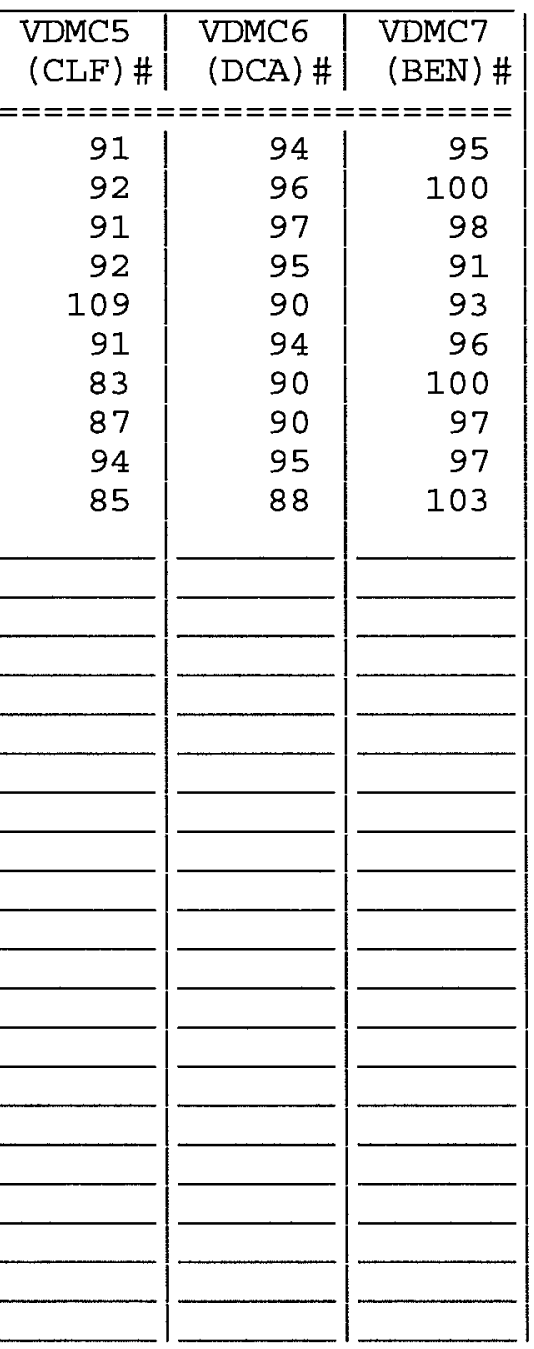

\# Column to be used to flag recovery values

* Values outside of contract required QC limits

Page 1 of 2 


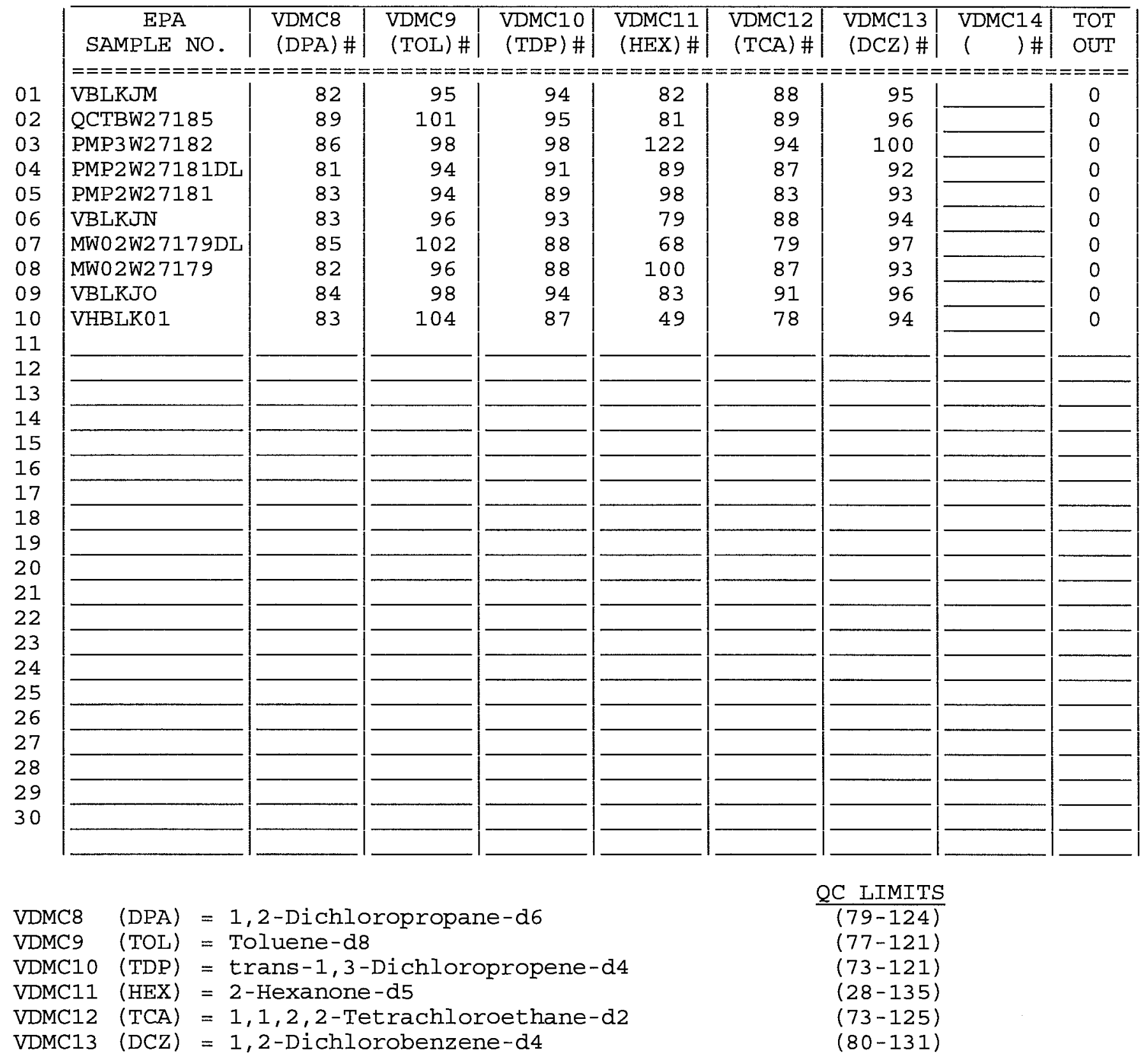

\# Column to be used to flag recovery values

* Values outside of contract required QC limits

Report 1,4-Dioxane-d8 for Low-Medium VOA analysis only Page 2 of 2 
$4 A$ - FORM IV VOA

VOLATILE METHOD BLANK SUMMARY

Lab Name: TESTAMERICA BURLINGTON

Contract: $8 \mathrm{E}-00302$

EPA SAMPLE NO.

VBLKJM

Lab Code: STLV Case No.: CNTRALIA Mod. Ref No.:

Lab File ID: JAQB03E

Lab Sample ID: VBLKJM

Instrument ID: J.i

Matrix: (SOIL/SED/WATER) Water

Date Analyzed: 04/08/2010

Level: (TRACE or LOW/MED) TRACE

Time Analyzed: 0919

GC Column: DB-624

ID : 0.53

(mm) Heated Purge: ( $\mathrm{Y} / \mathrm{N}) \mathrm{N}$

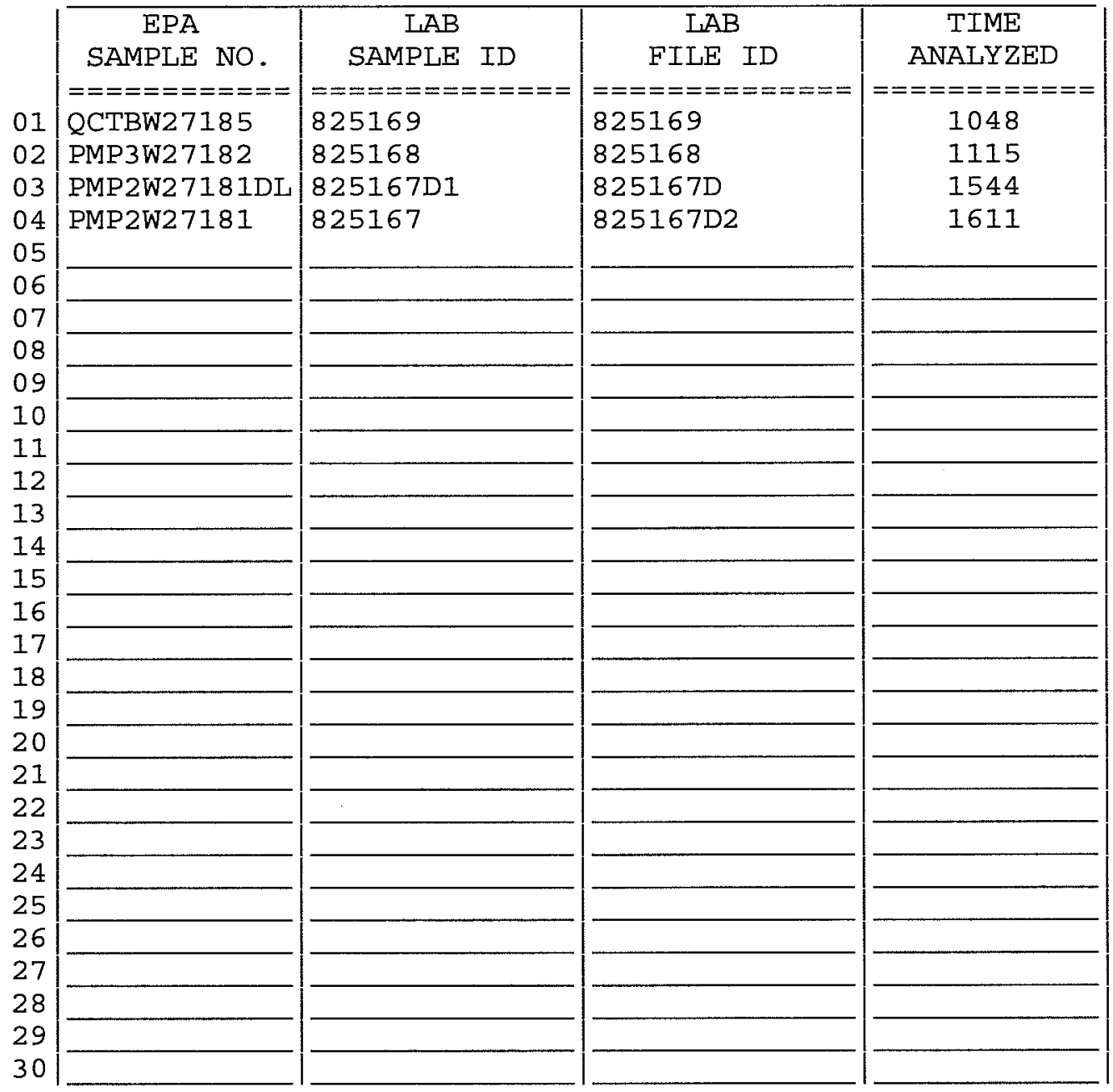

COMMENTS : 
$4 A$ - FORM IV VOA

VOLATILE METHOD BLANK SUMMARY
EPA SAMPLE NO.

VBLKJN

Lab Name: TESTAMERICA BURLINGTON

Contract: $8 \mathrm{E}-00302$

Lab Code: STLV Case No.: CNTRALIA Mod. Ref No.:

SDG No.: 136697

Lab File ID: JAQB02F

Instrument ID: J.i

Matrix: (SOIL/SED/WATER) Water

Level: (TRACE or LOW/MED) TRACE

GC Column: DB-624
ID $: 0.53$
Lab sample ID: VBLKJN

Date Analyzed: 04/09/2010

Time Analyzed: 0833

(mm) Heated Purge: ( $\mathrm{Y} / \mathrm{N}) \mathrm{N}$

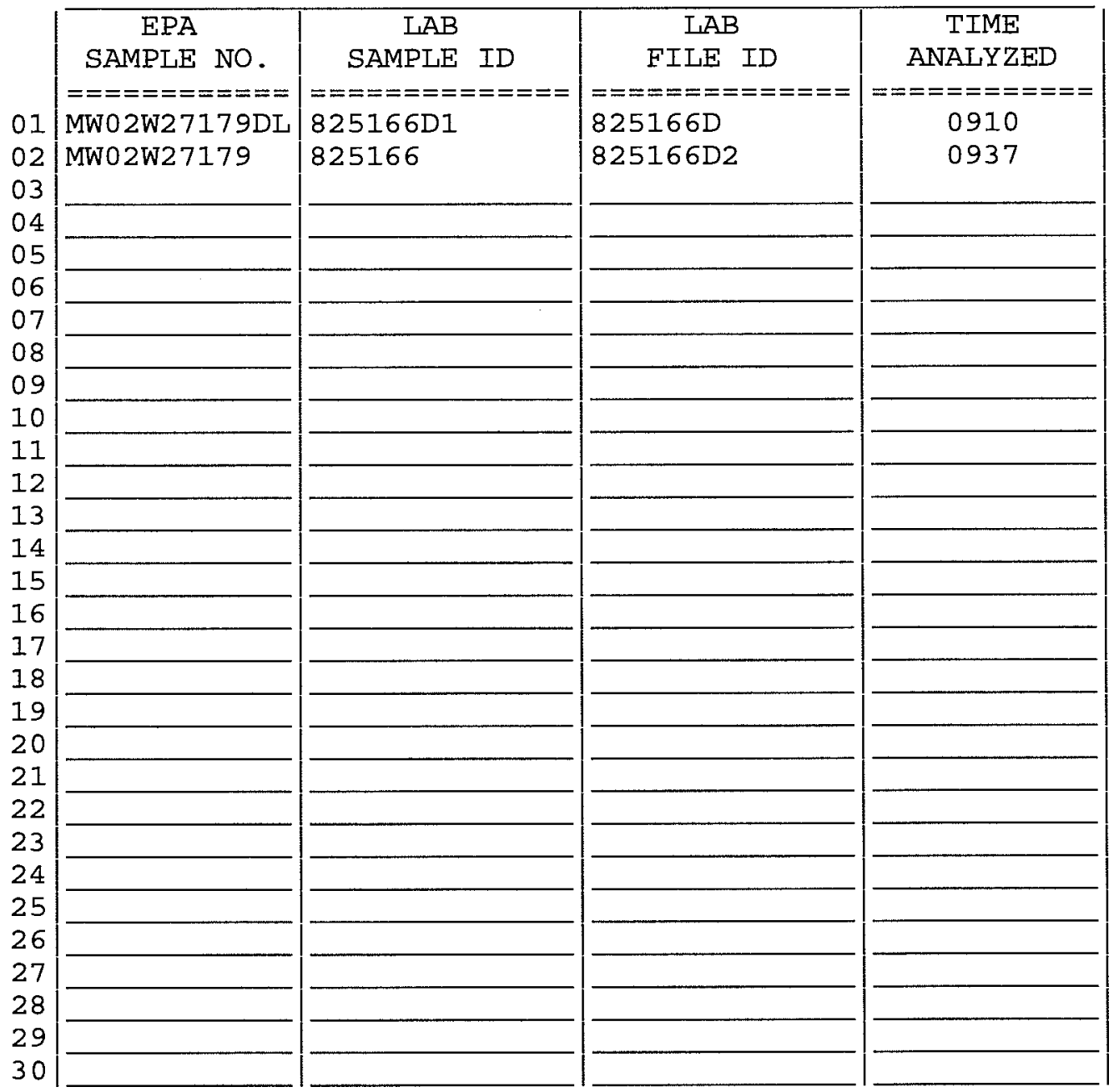

COMMENTS : 
$4 A$ - FORM IV VOA

VOLATILE METHOD BLANK SUMMARY
Lab Name: TESTAMERICA BURLINGTON

Contract: $8 \mathrm{E}-00302$

Lab Code: STLV Case No.: CNTRALIA Mod. Ref No.:
Lab File ID: JAQB02G

Instrument ID: J.i

Matrix: (SOIL/SED/WATER) Water

Level: (TRACE or LOW/MED) TRACE

GC Column: DB-624
ID $: 0.53$
EPA SAMPLE NO.

VBLKJO

Lab Sample ID: VBLKJO

Date Analyzed: 04/10/2010

Time Analyzed: 1042

(mm) Heated Purge: ( $\mathrm{Y} / \mathrm{N}) \mathrm{N}$

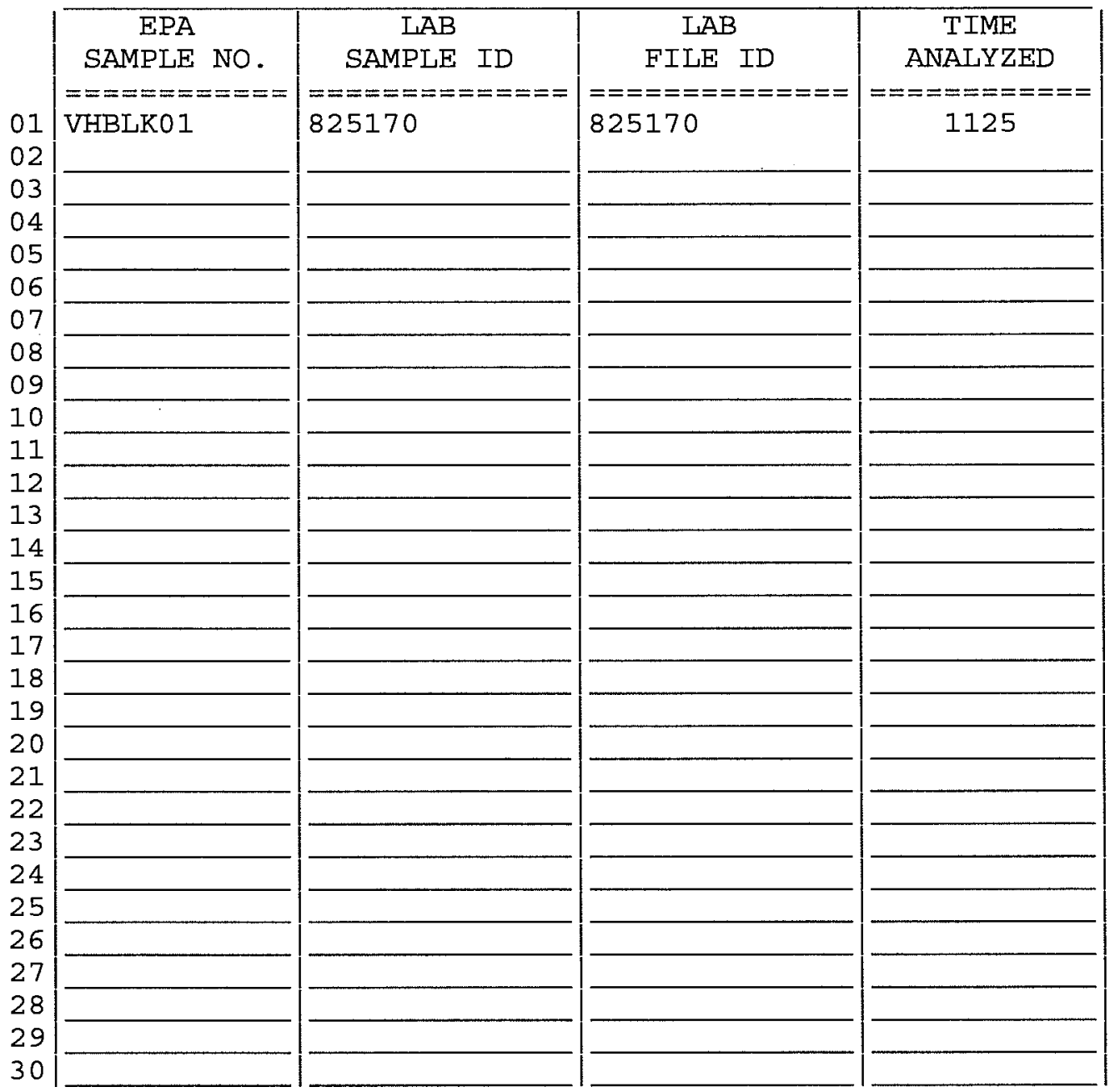

COMMENTS : 
$5 A$ - FORM V VOA

VOLATILE ORGANIC INSTRUMENT

PERFORMANCE CHECK

BROMOFLUOROBENZENE (BFB)
EPA SAMPLE NO.

BFBJG

Lab Name: TESTAMERICA BURLINGTON

Contract: $8 \mathrm{E}-00302$

Lab Code: STLV Case No.: CNTRALIA Mod. Ref No.:

SDG No.: 136697

Lab File ID: JAQ01PV

Instrument ID: J.i

GC Column: DB-624
BFB Injection Date: 04/05/2010

BFB Injection Time: 1223

\begin{tabular}{|c|c|c|}
\hline $\begin{array}{c}\mathrm{m} / \mathrm{e} \\
======\end{array}$ & 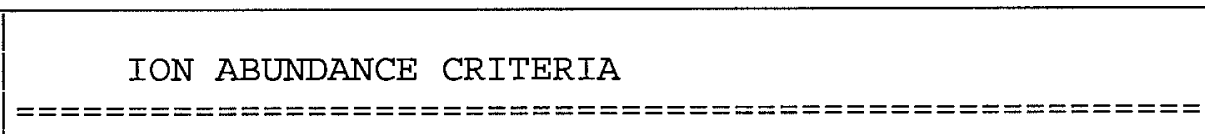 & $\begin{array}{c}\% \text { RELATIVE } \\
\text { ABUNDANCE } \\
===========\end{array}$ \\
\hline 50 & $15.0-40.0 \%$ of mass 95 & 18.0 \\
\hline 75 & $30.0-80.0 \%$ of mass 95 & 48.7 \\
\hline 95 & Base Peak, $100 \%$ relative abundance & 100.0 \\
\hline 96 & $5.0-9.0 \%$ of mass 95 & 7.0 \\
\hline 173 & Less than $2.0 \%$ of mass 174 & $0.0 \overline{1}$ \\
\hline 174 & $50.0-120.0 \%$ of mass 95 & 66.9 \\
\hline 175 & $5.0-9.0 \%$ of mass 174 & 6.01 \\
\hline 176 & $95.0-101.0 \%$ of mass $\overline{174}$ & $66.3(99.2) 1$ \\
\hline 177 & $5.0-9.0 \%$ of mass 176 & $4.5(6.8) 2$ \\
\hline
\end{tabular}

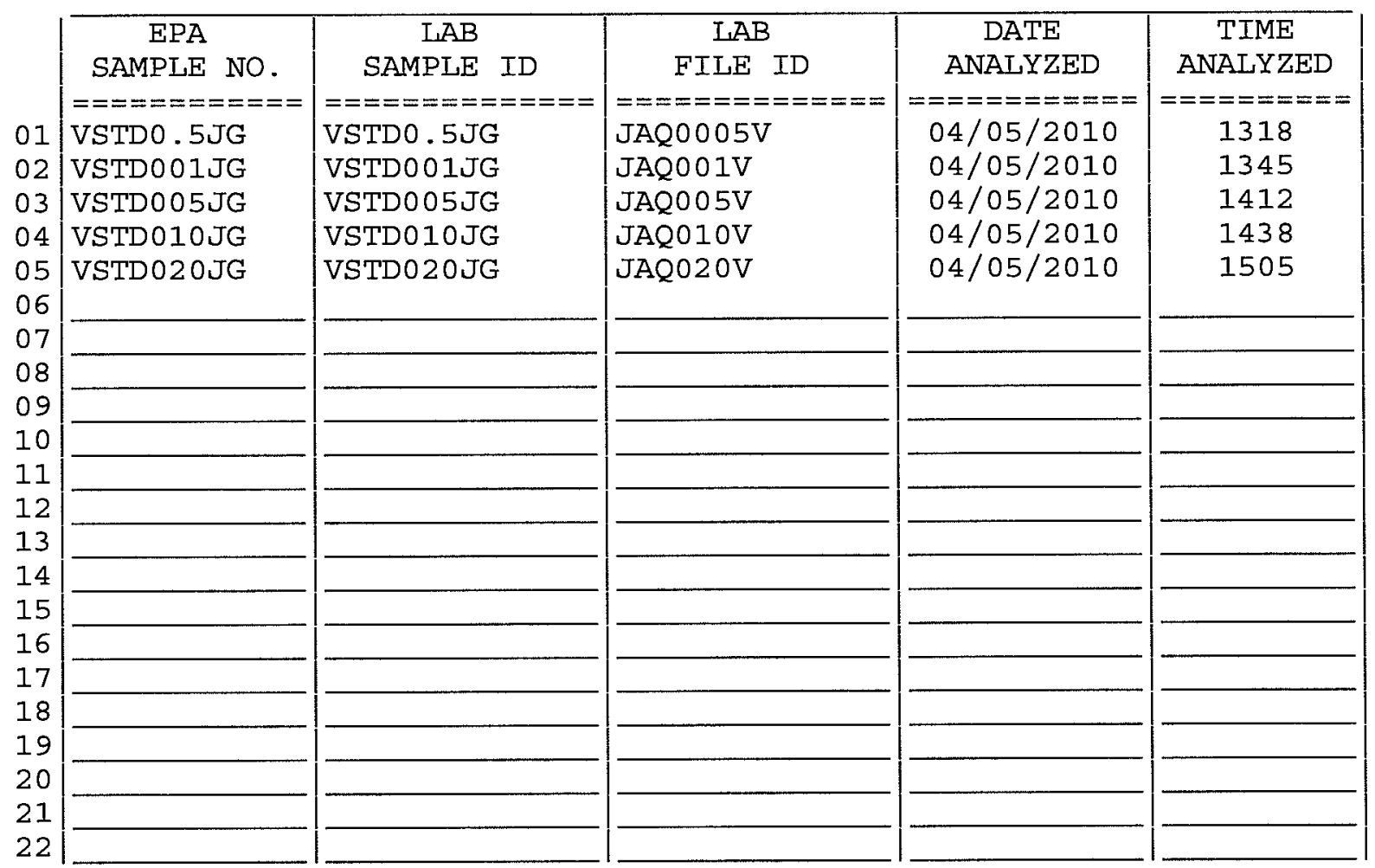


$5 A$ - FORM V VOA

VOLATILE ORGANIC INSTRUMENT

PERFORMANCE CHECK

BROMOFLUOROBENZENE (BFB)
EPA SAMPLE NO.

BFBJM

Lab Name: TESTAMERICA BURLINGTON

Contract: $8 \mathrm{E}-00302$

Lab Code: STLV Case No.: CNTRALIA Mod. Ref No.:

SDG No.: 136697

Lab File ID: JAQ13PV

Instrument ID: J.i

GC Column: DB-624
BFB Injection Date: 04/08/2010

BFB Injection Time: 0716

$$
\text { ID }: 0.53 \quad(\mathrm{~mm})
$$

\begin{tabular}{|c|c|c|}
\hline $\begin{array}{c}\mathrm{m} / \mathrm{e} \\
==== \\
50\end{array}$ & 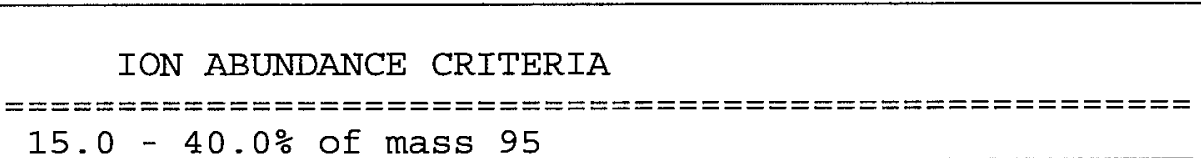 & $\begin{aligned} & \% \text { RELATIVE } \\
& \text { ABUNDANCE } \\
&============ \\
& 17.5\end{aligned}$ \\
\hline 75 & $30.0-80.0 \%$ of mass 95 & $48.9-$ \\
\hline 95 & Base Peak, $100 \%$ relative abundance & 100.0 \\
\hline 96 & $5.0-9.0 \%$ of mass 95 & 5.7 \\
\hline 173 & Less than $2.0 \%$ of mass 174 & $0.3) 1$ \\
\hline 174 & $50.0-120.0 \%$ of mass 95 & 69.8 \\
\hline 175 & $5.0-9.0 \%$ of mass 174 & $(7.8) 1$ \\
\hline 176 & $95.0-101.0 \%$ of mass $\overline{174}$ & $(98.7) 1$ \\
\hline 177 & $5.0-9.0 \%$ of mass 176 & $4.9(7.1) 2$ \\
\hline
\end{tabular}

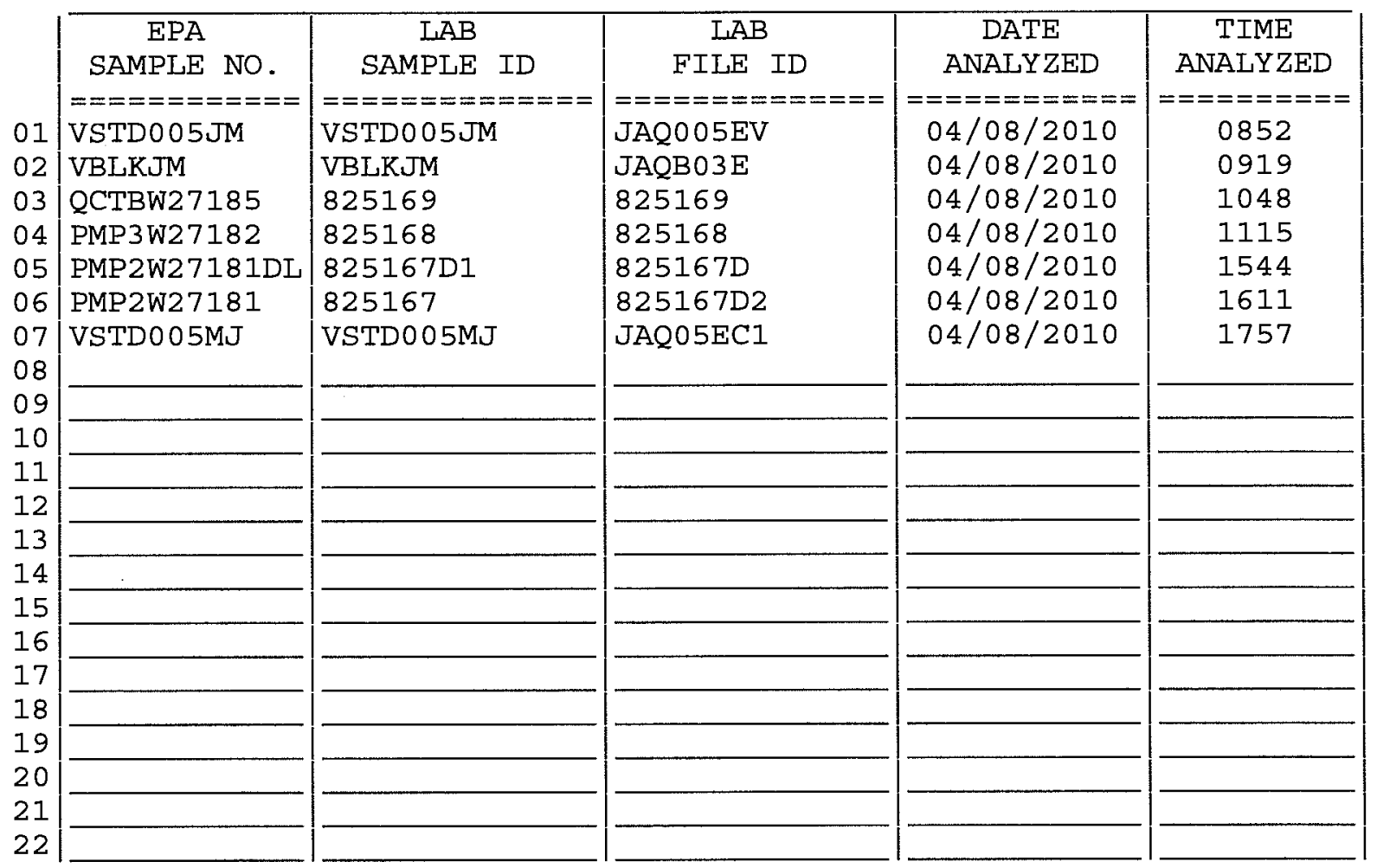


$5 A$ - FORM V VOA

VOLATILE ORGANIC INSTRUMENT

PERFORMANCE CHECK

BROMOFLUOROBENZENE (BFB)
EPA SAMPLE NO.

BFBJN

Contract: $8 \mathrm{E}-00302$

Lab Name: TESTAMERICA BURLINGTON

Lab Code: STLV Case No.: CNTRALIA Mod. Ref No.:

SDG NO.: 136697

BFB Injection Date: 04/09/2010

Lab File ID: JAQ15PV

BFB Injection Time: 0722

Instrument ID: J.i

ID $: 0.53 \quad(\mathrm{~mm})$

GC Column: DB-624

\begin{tabular}{|c|c|c|}
\hline $\begin{array}{c}\mathrm{m} / \mathrm{e} \\
======\end{array}$ & 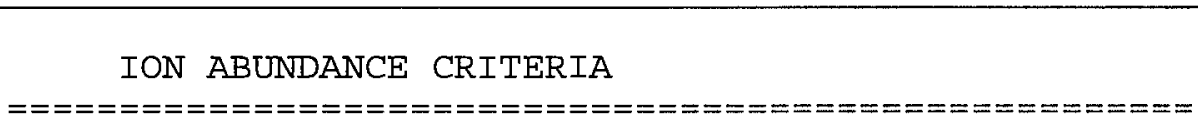 & $\begin{array}{c}\% \text { RELATIVE } \\
\text { ABUNDANCE } \\
===========\end{array}$ \\
\hline 50 & $15.0-40.0 \%$ of mass 95 & 16.3 \\
\hline 75 & $30.0-80.0 \%$ of mass 95 & 46.3 \\
\hline 95 & Base Peak, $100 \%$ relative abundance & 100.0 \\
\hline 96 & $5.0-9.0 \%$ of mass 95 & 6.0 \\
\hline 173 & Less than $2.0 \%$ of mass 174 & $0.7) 1$ \\
\hline 174 & $50.0-120.0 \%$ of mass 95 & 75.0 \\
\hline 175 & $5.0-9.0 \%$ of mass 174 & $6.1 T$ \\
\hline 176 & $95.0-101.0 \%$ of mass $\overline{174}$ & $74.5(99.3) 1$ \\
\hline 177 & $5.0-9.0 \%$ of mass 176 & $5.3(7.2) 2$ \\
\hline
\end{tabular}

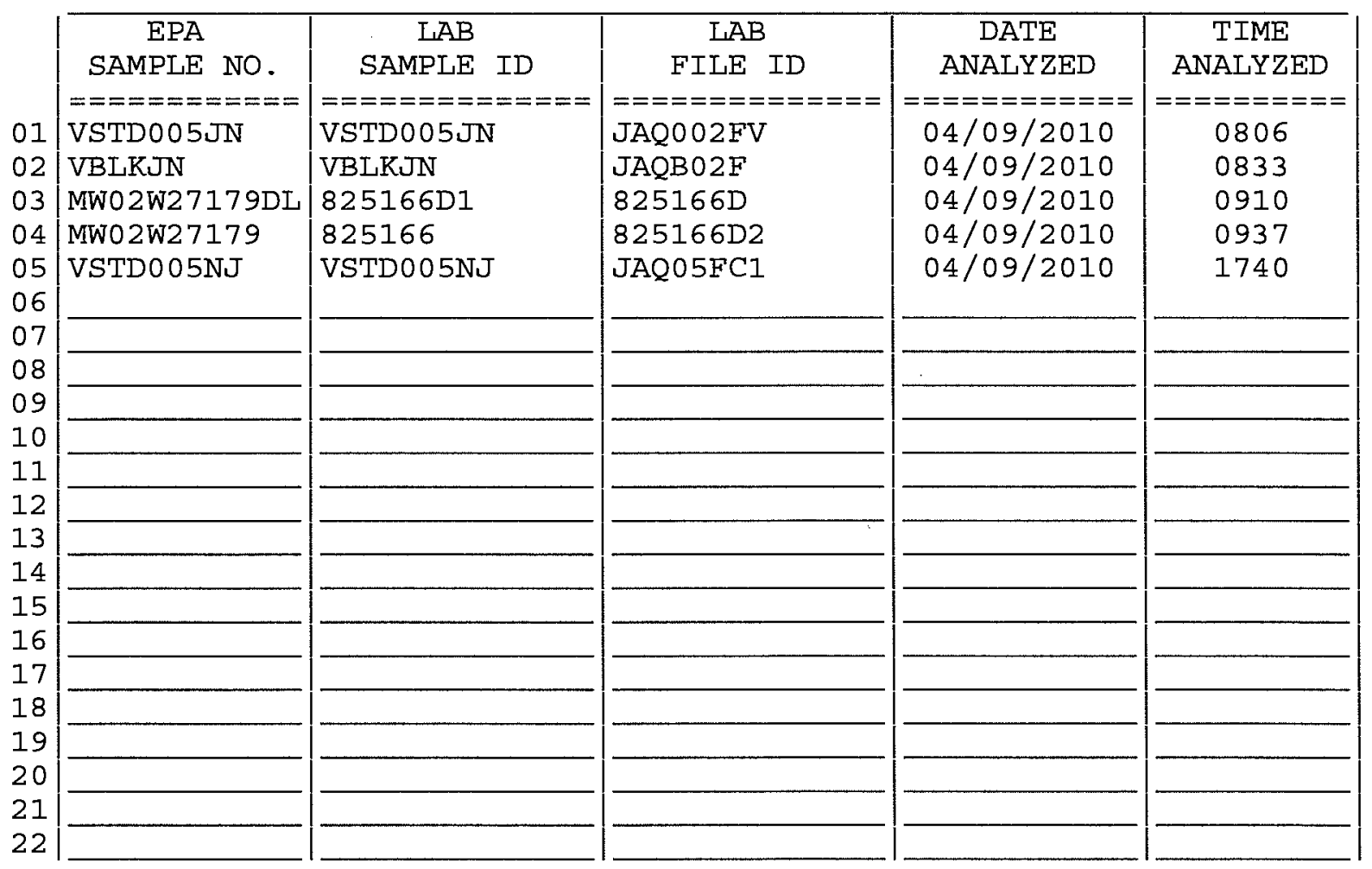


$5 A$ - FORM V VOA

VOLATILE ORGANIC INSTRUMENT

PERFORMANCE CHECK

BROMOFLUOROBENZENE (BFB)
EPA SAMPLE NO.

BFBJO
Lab Name: TESTAMERICA BURLINGTON

Contract: $8 E-00302$

Lab Code: STLV Case No.: CNTRALIA Mod. Ref No.:

Lab File ID: JAQ16PV

Instrument ID: J.i

GC Column: DB-624
ID : 0.53

(mm)

\begin{tabular}{|c|c|c|}
\hline $\begin{array}{c}\mathrm{m} / \mathrm{e} \\
====== \\
50\end{array}$ & $\begin{array}{c}\text { ION ABUNDANCE CRITERIA } \\
=================== \pm================================= \\
15.0-40.0 \% \text { of mass } 95\end{array}$ & $\begin{array}{c}\because \text { RELATIVE } \\
\text { ABUNDANCE } \\
============ \\
18.1\end{array}$ \\
\hline 75 & $30.0-80.0 \%$ of mass 95 & 48.8 \\
\hline 95 & Base Peak, $100 \%$ relative abundance & 100.0 \\
\hline 96 & $5.0-9.0 \%$ of mass 95 & 6.6 \\
\hline 173 & Less than $2.0 \%$ of mass 174 & $0.2) 1$ \\
\hline 174 & $50.0-120.0 \%$ of mass 95 & 65.8 \\
\hline 175 & $5.0-9.0 \%$ of mass 174 & $8.9) 1$ \\
\hline 176 & $95.0-101.0 \%$ of mass $\overline{174}$ & $(97.4) 1$ \\
\hline 177 & $5.0-9.0 \%$ of mass 176 & $(7.7) 2$ \\
\hline
\end{tabular}

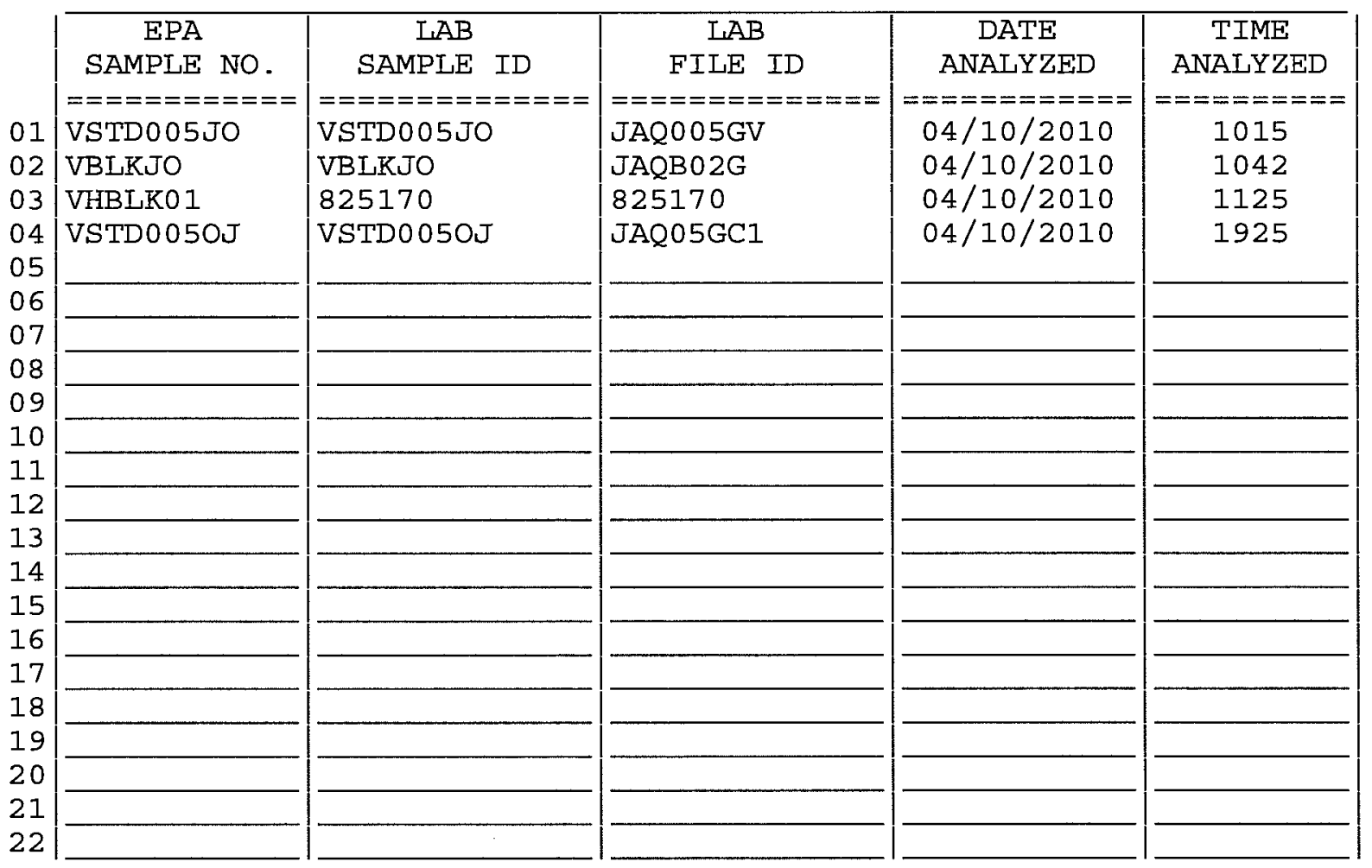


$6 A$ - FORM VI VOA-1

VOLATILE ORGANICS INITIAL CALIBRATION DATA

Lab Name: TESTAMERICA BURIINGTON

Lab Code: STLV Case No.: CNTRALIA

Instrument ID: J.i

Heated Purge: $(\mathrm{Y} / \mathrm{N}) \mathrm{N}$

Purge Volume: 25.0

GC Column: DB-624

(mL)
Contract: $8 E-00302$

Mod. Ref No.:

SDG No.: 136697

Calibration Date(s): 04/05/2010 04/05/2010

Calibration Time (s): $1318 \quad 1505$

ID : $0.53(\mathrm{~mm})$ Length: $75 \quad$ (m)

\begin{tabular}{|c|c|c|c|c|c|c|c|}
\hline $\begin{array}{l}\text { LAB FILE ID: } \\
\text { RRF5.0 = JAQ005V }\end{array}$ & $\begin{array}{l}.5=\mathrm{JA} \\
10=\mathrm{JAQ}\end{array}$ & $\begin{array}{l}20005 \mathrm{~V} \\
10 \mathrm{~V}\end{array}$ & & $\begin{array}{l}\mathrm{RF} 1.0= \\
\mathrm{RF} 20=\mathrm{J} Z\end{array}$ & $\begin{array}{l}\text { JAQ0 01V } \\
\text { AQ02 OV }\end{array}$ & & \\
\hline COMPOUND & RRF0. 5 & RRF1.0 & |RRF5. 0 & RRF10 & RRF20 & $\mathrm{RRF}$ & $\because \mathrm{RSD}$ \\
\hline 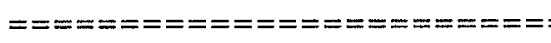 & $=======$ & $=======$ & $=======$ & $=======$ & $=======$ & $======$ & $:=====$ \\
\hline Dichlorodifluoromethane & 0.457 & 0.437 & 0.408 & 0.407 & 0.415 & 0.425 & 5.1 \\
\hline Chloromethane & 0.462 & 0.439 & 0.449 & 0.448 & 0.455 & 0.451 & 1.9 \\
\hline Vinyl chloride & 0.488 & 0.458 & 0.442 & 0.443 & 0.442 & 0.454 & 4.4 \\
\hline Bromomethane & 0.187 & 0.173 & 0.161 & 0.165 & 0.170 & 0.171 & 5.8 \\
\hline Chloroethane & 0.273 & 0.279 & 0.254 & 0.254 & 0.254 & 0.263 & 4.6 \\
\hline Trichlorofluoromethane & 0.524 & 0.522 & 0.507 & 0.501 & 0.502 & 0.511 & 2.2 \\
\hline 1,1-Dichloroethene & 0.320 & 0.313 & 0.300 & 0.296 & 0.301 & 0.306 & 3.3 \\
\hline 1,1,2-Trichloro- & & & & & & & \\
\hline 1,2,2-trifluoroethane & 0.348 & 0.330 & 0.315 & 0.310 & 0.314 & 0.323 & 4.8 \\
\hline Acetone & 0.016 & 0.015 & 0.013 & 0.013 & 0.013 & 0.014 & 10.1 \\
\hline Carbon disulfide & 1.128 & 0.982 & 0.959 & 0.931 & 0.933 & 0.986 & 8.3 \\
\hline Methyl acetate & 0.067 & 0.054 & 0.048 & 0.047 & 0.047 & 0.053 & 16.1 \\
\hline Methylene chloride & 0.262 & 0.252 & 0.250 & 0.242 & 0.246 & 0.250 & 3.0 \\
\hline trans-1,2-Dichloroethene & 0.336 & 0.344 & 0.321 & 0.313 & 0.314 & 0.326 & 4.3 \\
\hline Methyl tert-butyl ether & 0.403 & 0.409 & 0.394 & 0.383 & 0.395 & 0.397 & 2.5 \\
\hline 1,1-Dichloroethane & 0.606 & 0.610 & 0.595 & 0.588 & 0.584 & 0.597 & 1.9 \\
\hline cis-1,2-Dichloroethene & 0.328 & 0.321 & 0.316 & 0.305 & 0.308 & 0.316 & 3.0 \\
\hline 2-Butanone & 0.029 & 0.028 & 0.027 & 0.027 & 0.028 & 0.028 & 3.2 \\
\hline Bromochloromethane & 0.093 & 0.092 & 0.091 & 0.086 & 0.087 & 0.090 & 3.5 \\
\hline Chloroform & 0.531 & 0.499 & 0.491 & 0.488 & 0.493 & 0.500 & 3.5 \\
\hline 1,1,1-Trichloroethane & 0.663 & 0.647 & 0.610 & 0.604 & 0.599 & 0.625 & 4.6 \\
\hline Cyclohexane & 0.995 & 0.891 & 0.871 & 0.854 & 0.838 & 0.890 & 7.0 \\
\hline Carbon tetrachloride & 0.554 & 0.529 & 0.509 & 0.510 & 0.507 & 0.522 & 3.8 \\
\hline Benzene & 1.911 & 1.904 & 1.793 & 1.765 & 1.725 & 1.820 & 4.6 \\
\hline 1,2-Dichloroethane & 0.215 & 0.208 & 0.206 & 0.200 & 0.199 & 0.206 & 3.1 \\
\hline Trichloroethene & 0.440 & 0.432 & 0.423 & 0.420 & 0.419 & 0.427 & 2.1 \\
\hline Methylcyclohexane & 0.708 & 0.715 & 0.665 & 0.662 & 0.646 & 0.679 & 4.5 \\
\hline
\end{tabular}

Report 1,4-Dioxane for Low-Medium VOA analysis only 
$6 B$ - FORM VI VOA-2

VOLATILE ORGANICS INITIAL CALIBRATION DATA

Lab Name: TESTAMERICA BURLINGTON

Lab Code: STLV Case No.: CNTRALIA

Instrument ID: J.i

Heated Purge: $(\mathrm{Y} / \mathrm{N}) \mathrm{N}$

Purge Volume: 25.0

GC Column: DB-624

ID : $0.53 \quad(\mathrm{~mm})$

(mL)
Contract : $8 E-00302$

Mod. Ref No. :

SDG No.: 136697

Calibration Date(s): 04/05/2010 04/05/2010

Calibration Time (s): $1318 \quad 1505$

(mm) Length: 75 (m)

\begin{tabular}{|c|c|c|c|c|c|c|c|}
\hline $\begin{array}{l}\text { LAB FILE ID: } \\
\text { RRF5.0 = JAQ005V }\end{array}$ & $\begin{array}{l}.5=\mathrm{JAS} \\
0=\mathrm{JAQC}\end{array}$ & $\begin{array}{l}20005 \mathrm{~V} \\
10 \mathrm{~V}\end{array}$ & & $\begin{array}{l}2 F 1.0= \\
2 F 20=\mathrm{JZ}\end{array}$ & $\begin{array}{l}\text { JAQ001V } \\
\text { AQO20V }\end{array}$ & & \\
\hline COMPOUND & RRF0. 5 & RRF1 . 0 & RRF5. 0 & RRF10 & RRF20 & $\widehat{R R F}$ & $\because R S D$ \\
\hline 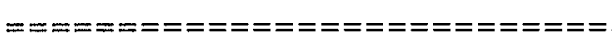 & $==-==-=$ & $=======$ & $==== \pm===$ & $========$ & $===== \pm==0$ & $=== \pm==$ & $====$ \\
\hline 1,2-Dichloropropane & 0.395 & 0.375 & 0.374 & 0.364 & 0.359 & 0.373 & 3.8 \\
\hline Bromodichloromethane & 0.404 & 0.377 & 0.383 & 0.382 & 0.386 & 0.386 & 2.6 \\
\hline cis-1,3-Dichloropropene & 0.507 & 0.506 & 0.496 & 0.496 & 0.495 & 0.500 & 1.2 \\
\hline 4-Methyl-2-pentanone & 0.096 & 0.098 & 0.097 & 0.095 & 0.097 & 0.096 & 1.0 \\
\hline Toluene & 2.055 & 1.965 & 1.891 & 1.836 & 1.831 & 1.916 & 4.9 \\
\hline trans-1,3-Dichloropropene & 0.343 & 0.336 & 0.348 & 0.343 & 0.345 & 0.343 & 1.3 \\
\hline $1,1,2$-Trichloroethane & 0.145 & 0.173 & 0.157 & 0.153 & 0.153 & 0.156 & 6.6 \\
\hline Tetrachloroethene & 0.361 & 0.353 & 0.329 & 0.327 & 0.329 & 0.340 & 4.6 \\
\hline 2-Hexanone & 0.059 & 0.062 & 0.061 & 0.061 & 0.062 & 0.061 & 2.0 \\
\hline Dibromochloromethane & 0.180 & 0.177 & 0.185 & 0.180 & 0.186 & 0.182 & 2.1 \\
\hline 1,2-Dibromoethane & 0.139 & 0.136 & 0.137 & 0.136 & 0.136 & 0.137 & 1.1 \\
\hline Chlorobenzene & 1.030 & 1.037 & 1.003 & 0.993 & 1.006 & 1.014 & 1.9 \\
\hline Ethylbenzene & 2.210 & 2.158 & 2.092 & 2.087 & 2.091 & 2.128 & 2.6 \\
\hline o-xylene & 0.780 & 0.732 & 0.712 & 0.713 & 0.711 & 0.730 & 4.0 \\
\hline $\mathrm{m}, \mathrm{p}$-xylene & 0.804 & 0.815 & 0.785 & 0.777 & 0.783 & 0.793 & 2.0 \\
\hline Styrene & 1.065 & 1.065 & 1.069 & 1.071 & 1.078 & 1.070 & 0.5 \\
\hline Bromoform & 0.173 & 0.191 & 0.197 & 0.198 & 0.195 & 0.191 & 5.4 \\
\hline Isopropylbenzene & 2.046 & 2.083 & 2.024 & 2.024 & 2.025 & 2.040 & 1.3 \\
\hline $1,1,2,2$-Tetrachloroethane & 0.144 & 0.152 & 0.144 & 0.147 & 0.146 & 0.147 & 2.1 \\
\hline 1,3-Dichlorobenzene & 1.688 & 1.654 & 1.599 & 1.626 & 1.601 & 1.634 & 2.3 \\
\hline 1,4-Dichlorobenzene & 1.655 & 1.589 & 1.520 & 1.528 & 1.510 & 1.560 & 3.9 \\
\hline 1,2-Dichlorobenzene & 1.282 & 1.238 & 1.179 & 1.183 & 1.162 & 1.209 & 4.1 \\
\hline 1,2-Dibromo-3-chloropropane & 0.063 & 0.052 & 0.044 & 0.045 & 0.044 & 0.050 & 16.8 \\
\hline $1,2,4$-Trichlorobenzene & 0.546 & 0.549 & 0.542 & 0.558 & 0.571 & 0.553 & 2.1 \\
\hline 1,2,3-Trichlorobenzene & 0.323 & 0.373 & 0.373 & 0.381 & 0.396 & 0.369 & 7.5 \\
\hline
\end{tabular}


6C - FORM VI VOA-3

VOLATILE ORGANICS INITIAL CALIBRATION DATA

Lab Name: TESTAMERICA BURLINGTON

Lab Code: STLV Case No.: CNTRAIIA

Instrument ID: J.i

Heated Purge: (Y/N) N

Purge Volume: 25.0

GC Column: $\mathrm{DB}-624$
Contract: $8 E-00302$

Mod. Ref No.:

SDG NO.: 136697

Calibration Date(s): 04/05/2010 04/05/2010

Calibration Time (s): $1318 \quad 1505$

(m工)

ID $: 0.53 \quad(\mathrm{~mm}) \quad$ Length: $75 \quad(\mathrm{~m})$

\begin{tabular}{|c|c|c|c|c|c|c|c|}
\hline $\begin{array}{l}\text { LAB FILE ID: } \\
\text { RRF5.0 = JAQ005V }\end{array}$ & $\begin{array}{l}0.5=\mathrm{JAC} \\
10=\mathrm{JAQC}\end{array}$ & $\begin{array}{l}20005 \mathrm{~V} \\
10 \mathrm{~V}\end{array}$ & & $\begin{array}{l}F 1.0= \\
F 20=J\end{array}$ & $\begin{array}{l}\mathrm{AQ} 001 \mathrm{~V} \\
\mathrm{Q} 020 \mathrm{~V}\end{array}$ & & \\
\hline COMPOUND & RRF0. 5 & RRF1. 0 & RRF5. 0 & RRF10 & RRF20 & $\overline{R R F}$ & $\% R S D$ \\
\hline 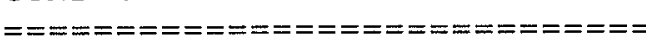 & $=======$ & $=======$ & $=== \pm===$ & $=======$ & $=======$ & $======$ & $====$ \\
\hline Vinyl chloride-d3 & 0.452 & 0.420 & 0.399 & 0.389 & 0.396 & 0.411 & 6.2 \\
\hline Chloroethane-d5 & 0.316 & 0.320 & 0.304 & 0.305 & 0.305 & 0.310 & 2.4 \\
\hline 1, 1-Di chloroethene-d2 & 0.724 & 0.703 & 0.670 & 0.657 & 0.660 & 0.683 & 4.3 \\
\hline 2-Butanone-d5 & 0.026 & 0.027 & 0.027 & 0.027 & 0.028 & 0.027 & 2.3 \\
\hline Chloroform-d & 0.571 & 0.539 & 0.540 & 0.525 & 0.528 & 0.541 & 3.3 \\
\hline 1,2-Dichloroethane-d4 & 0.172 & 0.176 & 0.167 & 0.165 & 0.165 & 0.169 & 3.0 \\
\hline Benzene-d6 & 1.846 & 1.824 & 1.730 & 1.708 & 1.689 & 1.760 & 4.0 \\
\hline 1,2 -Dichloropropane-d6 & 0.539 & 0.500 & 0.497 & 0.481 & 0.478 & 0.499 & 4.9 \\
\hline Toluene-d8 & 1.627 & 1.628 & 1.563 & 1.556 & 1.543 & 1.583 & 2.6 \\
\hline trans-1,3-Dichloropropene-d4 & 0.319 & 0.311 & 0.304 & 0.309 & 0.315 & 0.312 & 1.9 \\
\hline 2 -Hexanone-d5 & 0.032 & 0.033 & 0.034 & 0.034 & 0.034 & 0.033 & 2.9 \\
\hline $1,1,2,2$-Tetrachloroethane-d2 & 0.158 & 0.156 & 0.148 & 0.146 & 0.149 & 0.151 & 3.2 \\
\hline 1,2-Dichlorobenzene-d4 & 0.774 & 0.783 & 0.759 & 0.761 & 0.744 & 0.764 & 1.9 \\
\hline
\end{tabular}

Report 1,4-Dioxane-d8 for Low-Medium VOA analysis only 
$7 A$ - FORM VII VOA-1

VOLATILE CONTINUING CALIBRATION DATA

Lab Name: TESTAMERICA BURLINGTON

Contract: $8 \mathrm{E}-00302$

Lab Code: STLV Case No.: CNTRALIA Mod. Ref No.:

SDG No.: 136697

Instrument ID: J.i

Calibration Date: 04/08/2010 Time: 0852

Lab File ID: JAQ005EV

Init. Calib. Date(s): 04/05/2010 04/05/2010

EPA Sample No. (VSTD\#\#\#\#) : VSTD005JM Init. Calib. Time(s): $1318 \quad 1505$

Heated Purge: (Y/N)N GC Column: DB-624 ID: $0.53 \quad$ (mm) Length: $75 \quad$ (m)

Purge Volume: 25.0

$(\mathrm{mL})$

\begin{tabular}{|c|c|c|c|c|c|}
\hline COMPOUND & $\overline{\mathrm{RRF}}$ & RRF5. 0 & $\begin{array}{l}\text { MIN } \\
\text { RRF }\end{array}$ & $\div \mathrm{D}$ & $\mathrm{MAX} \div \mathrm{D}$ \\
\hline \multicolumn{6}{|c|}{ 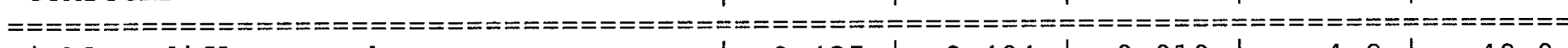 } \\
\hline Dichlorodifluoromethane & 0.425 & 0.404 & 0.010 & -4.9 & 40.0 \\
\hline Chloromethane & 0.451 & 0.462 & 0.010 & 2.6 & 40.0 \\
\hline Vinyl chloride & 0.454 & 0.455 & 0.100 & 0.0 & 30.0 \\
\hline Bromomethane & 0.171 & 0.161 & 0.100 & -6.2 & 30.0 \\
\hline Chloroethane & 0.263 & 0.257 & 0.010 & -2.3 & 40.0 \\
\hline Trichlorofluoromethane & 0.511 & 0.501 & 0.010 & -2.0 & 40.0 \\
\hline 1,1-Dichloroethene & 0.306 & 0.292 & 0.100 & -4.5 & 30.0 \\
\hline $1,1,2$-Trichloro-1,2,2-trifluoroethane & 0.323 & 0.317 & 0.010 & -1.8 & 40.0 \\
\hline Acetone & 0.014 & 0.012 & 0.010 & -13.8 & 40.0 \\
\hline Carbon disulfide & 0.986 & 0.925 & 0.010 & -6.2 & 40.0 \\
\hline Methyl acetate & 0.053 & 0.046 & 0.010 & -12.5 & 40.0 \\
\hline Methylene chloride & 0.250 & 0.229 & 0.010 & -8.7 & 40.0 \\
\hline trans-1,2-Dichloroethene & 0.326 & 0.316 & 0.010 & -3.1 & 40.0 \\
\hline Methyl tert-butyl ether & 0.397 & 0.350 & 0.010 & -11.9 & 40.0 \\
\hline 1,1-Dichloroethane & 0.597 & 0.583 & 0.200 & -2.2 & 30.0 \\
\hline cis-1,2-Dichloroethene & 0.316 & 0.299 & 0.010 & -5.4 & 40.0 \\
\hline 2-Butanone & 0.028 & 0.024 & 0.010 & -14.0 & 40.0 \\
\hline Bromochloromethane & 0.090 & 0.084 & 0.050 & -5.8 & 30.0 \\
\hline Chloroform & 0.500 & 0.476 & 0.200 & -4.8 & 30.0 \\
\hline $1,1,1$-Trichloroethane & 0.625 & 0.632 & 0.100 & 1.1 & 30.0 \\
\hline Cyclohexane & 0.890 & 0.886 & 0.010 & -0.4 & 40.0 \\
\hline Carbon tetrachloride & 0.522 & 0.532 & 0.100 & 1.9 & 30.0 \\
\hline Benzene & 1.820 & 1.771 & 0.400 & -2.7 & 30.0 \\
\hline 1,2-Dichloroethane & 0.206 & 0.191 & 0.100 & -7.2 & 30.0 \\
\hline Trichloroethene & 0.427 & 0.432 & 0.300 & 1.3 & 30.0 \\
\hline Methylcyclohexane & 0.679 & 0.694 & 0.010 & 2.1 & 40.0 \\
\hline
\end{tabular}

Report 1,4-Dioxane for Low-Medium VOA analysis only

SOMO1.2 
7B - FORM VII VOA-2

VOLATILE CONTINUING CALIBRATION DATA

Lab Name: TESTAMERICA BURLINGTON

Contract: $8 \mathrm{E}-00302$

Lab Code: STLV Case No.: CNTRALIA Mod. Ref No.:

SDG No.: 136697

Instrument ID: J.i

Calibration Date: 04/08/2010 Time: 0852

Lab File ID: JAQ005EV

Init. Calib. Date(s): 04/05/2010 04/05/2010

EPA Sample No. (VSTD\#\#\#\#\#): VSTD005JM Init. Calib. Time(s): $1318 \quad 1505$

Heated Purge: ( $\mathrm{Y} / \mathrm{N}) \mathrm{N}$ GC Column: $\mathrm{DB}-624$ ID: $0.53 \quad$ (mm) Length: $75 \quad$ (m)

Purge Volume: 25.0

$(\mathrm{mL})$

\begin{tabular}{|c|c|c|c|c|c|}
\hline COMPOUND & $\overline{\mathrm{RRF}}$ & RRF5. 0 & $\begin{array}{l}\text { MIN } \\
\text { RRF }\end{array}$ & $\div \mathrm{D}$ & MAX $\because D$ \\
\hline \multicolumn{6}{|c|}{ 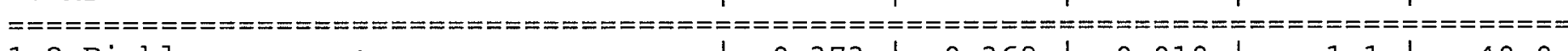 } \\
\hline 1,2-Dichloropropane & 0.373 & 0.369 & 0.010 & -1.1 & 40.0 \\
\hline Bromodichloromethane & 0.386 & 0.374 & 0.200 & -3.1 & 30.0 \\
\hline cis-1,3-Dichloropropene & 0.500 & 0.483 & 0.200 & -3.3 & 30.0 \\
\hline 4-Methyl-2-pentanone & 0.096 & 0.089 & 0.010 & -8.1 & 40.0 \\
\hline Toluene & 1.916 & 1.878 & 0.400 & -2.0 & 30.0 \\
\hline trans-1,3-Dichloropropene & 0.343 & 0.341 & 0.100 & -0.6 & 30.0 \\
\hline 1,1,2-Trichloroethane & 0.156 & 0.148 & 0.100 & -5.1 & 30.0 \\
\hline Tetrachloroethene & 0.340 & 0.342 & 0.100 & 0.6 & 30.0 \\
\hline 2-Hexanone & 0.061 & 0.057 & 0.010 & -7.0 & 40.0 \\
\hline Dibromochloromethane & 0.182 & 0.173 & 0.100 & -4.6 & 30.0 \\
\hline 1,2-Dibromoethane & 0.137 & 0.130 & 0.010 & -5.1 & 30.0 \\
\hline Chlorobenzene & 1.014 & 1.002 & 0.500 & -1.2 & 30.0 \\
\hline Ethylbenzene & 2.128 & 2.127 & 0.100 & -0.0 & 30.0 \\
\hline o-Xylene & 0.730 & 0.713 & 0.300 & -2.3 & 30.0 \\
\hline $\mathrm{m}, \mathrm{p}$-Xylene & 0.793 & 0.786 & 0.300 & -0.8 & 30.0 \\
\hline styrene & 1.070 & 1.046 & 0.300 & -2.2 & 30.0 \\
\hline Bromoform & 0.191 & 0.185 & 0.050 & -3.1 & 30.0 \\
\hline Isopropylbenzene & 2.040 & 2.054 & 0.010 & 0.7 & 40.0 \\
\hline $1,1,2,2$-Tetrachloroethane & 0.147 & 0.138 & 0.100 & -6.2 & 30.0 \\
\hline 1,3-Dichlorobenzene & 1.634 & 1.554 & 0.400 & -4.9 & 30.0 \\
\hline 1,4-Dichlorobenzene & 1.560 & 1.503 & 0.400 & -3.7 & 30.0 \\
\hline 1,2-Dichlorobenzene & 1.209 & 1.117 & 0.400 & -7.6 & 30.0 \\
\hline 1,2-Dibromo-3-chloropropane & 0.050 & 0.039 & 0.010 & -21.1 & 40.0 \\
\hline $1,2,4$-Trichlorobenzene & 0.553 & 0.516 & 0.200 & -6.6 & 30.0 \\
\hline 1,2,3-Trichlorobenzene & 0.369 & 0.351 & 0.200 & -4.9 & 30.0 \\
\hline
\end{tabular}

SOM01.2 
$7 C$ - FORM VII VOA-3

VOLATILE CONTINUING CALIBRATION DATA

Lab Name: TESTAMERICA BURLINGTON

Contract: $8 \mathrm{E}-00302$

Lab Code: STLV Case No.: CNTRALIA Mod. Ref No.:

SDG No.: 136697

Instrument ID: J.i

Calibration Date: 04/08/2010 Time: 0852

Lab File ID: JAQ005EV

Init. Calib. Date(s): 04/05/2010 04/05/2010

EPA Sample No. (VSTD\#\#\#\#\#): VSTD005JM

Init. Calib. Time(s): 1318

1505

Heated Purge: (Y/N)N GC Column: DB-62

ID : 0.53

(mm) Length: 75

(m)

Purge Volume: 25.0

$(\mathrm{mL})$

\begin{tabular}{|c|c|c|c|c|c|}
\hline COMPOUND & $\overline{\mathrm{RRF}}$ & RRF5. 0 & $\begin{array}{l}\text { MIN } \\
\text { RRF }\end{array}$ & $\div \mathrm{D}$ & $\mathrm{MAX} \div \mathrm{D}$ \\
\hline \multirow{2}{*}{\multicolumn{6}{|c|}{ 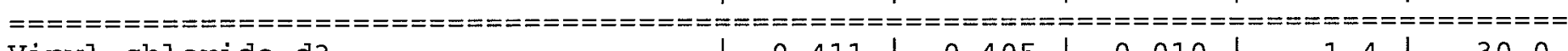 }} \\
\hline Vinyl chloride-d3 & & & & & \\
\hline Chloroethane-d5 & 0.310 & 0.311 & 0.010 & 0.2 & 40.0 \\
\hline 1,1-Dichloroethene-d2 & 0.683 & 0.674 & 0.010 & -1.3 & 30.0 \\
\hline 2-Butanone-d5 & 0.027 & 0.023 & 0.010 & -12.7 & 40.0 \\
\hline Chloroform-d & 0.541 & 0.505 & 0.010 & -6.6 & 30.0 \\
\hline 1,2-Dichloroethane-d4 & 0.169 & 0.160 & 0.010 & -5.4 & 30.0 \\
\hline Benzene-d6 & 1.760 & 1.732 & 0.400 & -1.6 & 30.0 \\
\hline 1,2-Dichloropropane-d6 & 0.499 & 0.432 & 0.010 & -13.5 & 40.0 \\
\hline Toluene-d8 & 1.583 & 1.576 & 0.010 & -0.5 & 30.0 \\
\hline trans-1,3-Dichloropropene-d4 & 0.312 & 0.306 & 0.010 & -1.8 & 30.0 \\
\hline 2-Hexanone-d5 & 0.033 & 0.028 & 0.010 & -14.8 & 40.0 \\
\hline $1,1,2,2$-Tetrachloroethane-d2 & 0.151 & 0.140 & 0.010 & -7.7 & 30.0 \\
\hline 1,2-Dichlorobenzene-d4 & 0.764 & 0.723 & 0.010 & -5.4 & 30.0 \\
\hline
\end{tabular}

Report 1,4-Dioxane-d8 for Low-Medium VOA analysis only 
$7 A$ - FORM VII VOA-1

VOLATILE CONTINUING CALIBRATION DATA

Lab Name: TESTAMERICA BURLINGTON

Contract: $8 \mathrm{E}-00302$

Lab Code: STLV Case No.: CNTRALIA Mod. Ref No.:

SDG No.: 136697

Instrument ID: J.i

Calibration Date: 04/08/2010 Time: 1757

Lab File ID: JAQ05EC1

Init. Calib. Date(s): 04/05/2010 04/05/2010

EPA Sample No.(VSTD\#\#\#\#): VSTD005MJ Init. Calib. Time(s): 131811505

Heated Purge: ( $\mathrm{Y} / \mathrm{N}) \mathrm{N}$ GC Column: DB-624 ID: $0.53 \quad$ (mm) Length: $75 \quad$ (m)

Purge Volume: 25.0

$(\mathrm{mL})$

\begin{tabular}{|c|c|c|c|c|c|}
\hline COMPOUND & $\overline{\mathrm{RRF}}$ & RRF5. 0 & $\begin{array}{l}\text { MIN } \\
\text { RRF }\end{array}$ & $\div D$ & MAX $\div D$ \\
\hline \multirow{2}{*}{\multicolumn{6}{|c|}{ 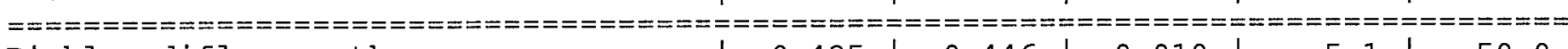 }} \\
\hline Dichlorodifluoromethane & 0.425 & 0.446 & 0.010 & & 50.0 \\
\hline Chloromethane & 0.451 & 0.503 & 0.010 & 11.7 & 50.0 \\
\hline Vinyl chloride & 0.454 & 0.492 & 0.010 & 8.3 & 50.0 \\
\hline Bromomethane & 0.171 & 0.173 & 0.010 & 1.2 & 50.0 \\
\hline Chloroethane & 0.263 & 0.283 & 0.010 & 7.8 & 50.0 \\
\hline Trichlorofluoromethane & 0.511 & 0.545 & 0.010 & 6.5 & 50.0 \\
\hline 1,1-Dichloroethene & 0.306 & 0.317 & 0.010 & 3.5 & 50.0 \\
\hline 1,1,2-Trichloro-1,2,2-trifluoroethane & 0.323 & 0.344 & 0.010 & 6.4 & 50.0 \\
\hline Acetone & 0.014 & 0.011 & 0.010 & -19.4 & 50.0 \\
\hline Carbon disulfide & 0.986 & 1.012 & 0.010 & 2.6 & 50.0 \\
\hline Methyl acetate & 0.053 & 0.047 & 0.010 & -10.0 & 50.0 \\
\hline Methylene chloride & 0.250 & 0.254 & 0.010 & 1.6 & 50.0 \\
\hline trans-1,2-Dichloroethene & 0.326 & 0.342 & 0.010 & 5.2 & 50.0 \\
\hline Methyl tert-butyl ether & 0.397 & 0.367 & 0.010 & -7.6 & 50.0 \\
\hline 1,1-Dichloroethane & 0.597 & 0.626 & 0.010 & 5.0 & 50.0 \\
\hline cis-1,2-Dichloroethene & 0.316 & 0.324 & 0.010 & 2.7 & 50.0 \\
\hline 2-Butanone & 0.028 & 0.025 & 0.010 & -9.6 & 50.0 \\
\hline Bromochloromethane & 0.090 & 0.091 & 0.010 & 1.4 & 50.0 \\
\hline Chloroform & 0.500 & 0.524 & 0.010 & 4.8 & 50.0 \\
\hline 1,1,1-Trichloroethane & 0.625 & 0.666 & 0.010 & 6.7 & 50.0 \\
\hline Cyclohexane & 0.890 & 0.940 & 0.010 & 5.7 & 50.0 \\
\hline Carbon tetrachloride & 0.522 & 0.553 & 0.010 & 6.0 & 50.0 \\
\hline Benzene & 1.820 & 1.879 & 0.010 & 3.2 & 50.0 \\
\hline 1,2-Dichloroethane & 0.206 & 0.210 & 0.010 & 2.1 & 50.0 \\
\hline Trichloroethene & 0.427 & 0.456 & 0.010 & 6.8 & 50.0 \\
\hline Methylcyclohexane & 0.679 & 0.724 & 0.010 & 6.5 & 50.0 \\
\hline
\end{tabular}

Report 1,4-Dioxane for Low-Medium VOA analysis only

SOM01.2 
7B - FORM VII VOA-2

VOLATILE CONTINUING CALIBRATION DATA

Lab Name: TESTAMERICA BURLINGTON

Contract: $8 \mathrm{E}-00302$

Lab Code: STLV Case No.: CNT'RALIA Mod. Ref No.:

SDG NO.: 136697

Instrument ID: J.i

Calibration Date: 04/08/2010 Time: 1757

Lab File ID: JAQ05EC1

Init. Calib. Date(s): 04/05/2010 04/05/2010

EPA Sample No. (VSTD\#\#\#\#\#): VSTD005MJ Init. Calib. Time(s): $1318 \quad 1505$

Heated Purge: (Y/N)N GC Column: DB-624 ID: $0.53 \quad$ (mm) Length: $75 \quad$ (m)

Purge Volume: 25.0

$(\mathrm{mL})$

\begin{tabular}{|c|c|c|c|c|c|}
\hline COMPOUND & $\overline{\mathrm{RRF}}$ & RRF5. 0 & $\begin{array}{l}\text { MIN } \\
\text { RRF }\end{array}$ & $\div \mathrm{D}$ & MAX $\div D$ \\
\hline \multicolumn{6}{|c|}{ 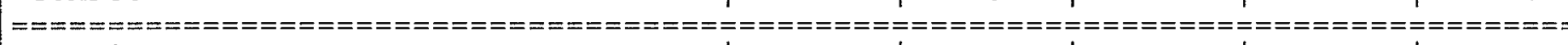 } \\
\hline 1,2-Dichloropropane & 0.373 & 0.386 & 0.010 & 3.3 & 50.0 \\
\hline Bromodichloromethane & 0.386 & 0.392 & 0.010 & 1.6 & 50.0 \\
\hline cis-1,3-Dichloropropene & 0.500 & 0.502 & 0.010 & 0.5 & 50.0 \\
\hline 4-Methyl-2-pentanone & 0.096 & 0.092 & 0.010 & -4.4 & 50.0 \\
\hline Toluene & 1.916 & 1.972 & 0.010 & 2.9 & 50.0 \\
\hline trans-1,3-Dichloropropene & 0.343 & 0.345 & 0.010 & 0.7 & 50.0 \\
\hline 1,1,2-Trichloroethane & 0.156 & 0.157 & 0.010 & 0.2 & 50.0 \\
\hline Tetrachloroethene & 0.340 & 0.357 & 0.010 & 5.2 & 50.0 \\
\hline 2-Hexanone & 0.061 & 0.059 & 0.010 & -3.6 & 50.0 \\
\hline Dibromochloromethane & 0.182 & 0.183 & 0.010 & 0.8 & 50.0 \\
\hline 1,2-Dibromoethane & 0.137 & 0.136 & 0.010 & -0.9 & 50.0 \\
\hline Chlorobenzene & 1.014 & 1.036 & 0.010 & 2.2 & 50.0 \\
\hline Ethylbenzene & 2.128 & 2.234 & 0.010 & 5.0 & 50.0 \\
\hline o-Xylene & 0.730 & 0.754 & 0.010 & 3.3 & 50.0 \\
\hline $\mathrm{m}, \mathrm{p}$-XYlene & 0.793 & 0.826 & 0.010 & 4.2 & 50.0 \\
\hline styrene & 1.070 & 1.088 & 0.010 & 1.8 & 50.0 \\
\hline Bromoform & 0.191 & 0.191 & 0.010 & 0.3 & 50.0 \\
\hline Isopropylbenzene & 2.040 & 2.155 & 0.010 & 5.7 & 50.0 \\
\hline $1,1,2,2$-Tetrachloroethane & 0.147 & 0.139 & 0.010 & -4.9 & 50.0 \\
\hline 1,3-Dichlorobenzene & 1.634 & 1.655 & 0.010 & 1.3 & 50.0 \\
\hline 1,4-Dichlorobenzene & 1.560 & 1.563 & 0.010 & 0.2 & 50.0 \\
\hline 1,2-Dichlorobenzene & 1.209 & 1.214 & 0.010 & 0.5 & 50.0 \\
\hline 1,2-Dibromo-3-chloropropane & 0.050 & 0.041 & 0.010 & -17.9 & 50.0 \\
\hline $1,2,4$-Trichlorobenzene & 0.553 & 0.540 & 0.010 & -2.4 & 50.0 \\
\hline 1,2,3-Trichlorobenzene & 0.369 & 0.360 & 0.010 & -2.4 & 50.0 \\
\hline
\end{tabular}

SOM01.2 
$7 C$ - FORM VII VOA-3

VOLATILE CONTINUING CALIBRATION DATA

Lab Name: TESTAMERICA BURLINGTON

Contract: $8 \mathrm{E}-00302$

Lab Code: STLV Case No.: CNTRALIA Mod. Ref No.:

SDG NO.: 136697

Instrument ID: J.i

Calibration Date: 04/08/2010 Time: 1757

Lab File ID: JAQ05EC1

Init. Calib. Date(s): 04/05/2010 04/05/2010

EPA Sample No. (VSTD\#\#\#\#\#): VSTD005MJ

Init. Calib. Time(s): $1318 \quad 1505$

Heated Purge: (Y/N)N GC Column: DB-624 ID: $0.53 \quad$ (mm) Length: 75

(m)

Purge Volume: 25.0

(mL)

\begin{tabular}{|c|c|c|c|c|c|}
\hline COMPOUND & $\overline{\mathrm{RRF}}$ & RRF5. 0 & $\begin{array}{l}\text { MIN } \\
\text { RRF }\end{array}$ & $\div \mathrm{D}$ & MAX $\div D$ \\
\hline \multirow{2}{*}{\multicolumn{6}{|c|}{ 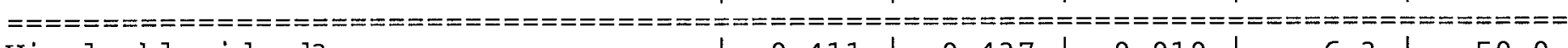 }} \\
\hline & & & & & 50.0 \\
\hline Chloroethane-d5 & 0.310 & 0.332 & 0.010 & 7.0 & 50.0 \\
\hline 1,1-Dichloroethene- $d 2$ & 0.683 & 0.726 & 0.010 & 6.3 & 50.0 \\
\hline 2-Butanone-d5 & 0.027 & 0.025 & 0.010 & -7.4 & 50.0 \\
\hline Chloroform-d & 0.541 & 0.549 & 0.010 & 1.6 & 50.0 \\
\hline 1,2-Dichloroethane-d4 & 0.169 & 0.172 & 0.010 & 1.8 & 50.0 \\
\hline Benzene-d6 & 1.760 & 1.812 & 0.010 & 3.0 & 50.0 \\
\hline 1,2-Dichloropropane-d6 & 0.499 & 0.503 & 0.010 & 0.9 & 50.0 \\
\hline Toluene-d8 & 1.583 & 1.674 & 0.010 & 5.7 & 50.0 \\
\hline trans-1,3-Dichloropropene-d4 & 0.312 & 0.313 & 0.010 & 0.6 & 50.0 \\
\hline 2-Hexanone-d5 & 0.033 & 0.029 & 0.010 & -13.1 & 50.0 \\
\hline $1,1,2,2$-Tetrachloroethane-d2 & 0.151 & 0.144 & 0.010 & -4.8 & 50.0 \\
\hline 1,2-Dichlorobenzene-d4 & 0.764 & 0.773 & 0.010 & 1.2 & 50.0 \\
\hline
\end{tabular}

Report 1,4-Dioxane-d8 for Low-Medium VOA analysis only 
$7 A$ - FORM VII VOA-1

VOLATILE CONTINUING CALIBRATION DATA

Lab Name: TESTAMERICA BURLINGTON

Contract: $8 E-00302$

Lab Code: STLV Case No.: CNTRALIA Mod. Ref No.:

SDG No.: 136697

Instrument ID: J.i

Calibration Date: 04/09/2010 Time: 0806

Lab File ID: JAQ002FV

Init. Calib. Date(s) : 04/05/2010 04/05/2010

EPA Sample No. (VSTD\#\#\#\#\#): VSTD005JN Init. Calib. Time(s): $1318 \quad 1505$

Heated Purge: (Y/N)N GC Column: DB-624 ID: $0.53 \quad$ (mm) Length: 75 (m)

Purge Volume: 25.0

$(\mathrm{mL})$

\begin{tabular}{|c|c|c|c|c|c|}
\hline COMPOUND & $\overline{\mathrm{RRF}}$ & RRF5. 0 & $\begin{array}{l}\text { MIN } \\
\text { RRF }\end{array}$ & $\because \mathrm{D}$ & $\mathrm{MAX} \div \mathrm{D}$ \\
\hline \multicolumn{6}{|c|}{ 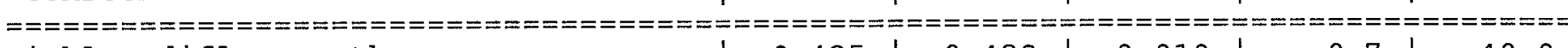 } \\
\hline Dichlorodifluoromethane & 0.425 & 0.428 & 0.010 & 0.7 & 40.0 \\
\hline Chloromethane & 0.451 & 0.475 & 0.010 & 5.5 & 40.0 \\
\hline Vinyl chloride & 0.454 & 0.480 & 0.100 & 5.6 & 30.0 \\
\hline Bromomethane & 0.171 & 0.182 & 0.100 & 6.1 & 30.0 \\
\hline Chloroethane & 0.263 & 0.275 & 0.010 & 4.6 & 40.0 \\
\hline Trichlorofluoromethane & 0.511 & 0.527 & 0.010 & 3.0 & 40.0 \\
\hline 1,1-Dichloroethene & 0.306 & 0.299 & 0.100 & -2.2 & 30.0 \\
\hline $1,1,2$-Trichloro-1,2,2-trifluoroethane & 0.323 & 0.326 & 0.010 & 0.8 & 40.0 \\
\hline Acetone & 0.014 & 0.012 & 0.010 & -15.9 & 40.0 \\
\hline Carbon disulfide & 0.986 & 0.964 & 0.010 & -2.3 & 40.0 \\
\hline Methyl acetate & 0.053 & 0.048 & 0.010 & -9.2 & 40.0 \\
\hline Methylene chloride & 0.250 & 0.243 & 0.010 & -2.8 & 40.0 \\
\hline trans-1,2-Dichloroethene & 0.326 & 0.319 & 0.010 & -2.0 & 40.0 \\
\hline Methyl tert-butyl ether & 0.397 & 0.356 & 0.010 & -10.2 & 40.0 \\
\hline 1,1-Dichloroethane & 0.597 & 0.597 & 0.200 & 0.0 & 30.0 \\
\hline cis-1,2-Dichloroethene & 0.316 & 0.312 & 0.010 & -1.1 & 40.0 \\
\hline 2-Butanone & 0.028 & 0.025 & 0.010 & -10.8 & 40.0 \\
\hline Bromochloromethane & 0.090 & 0.087 & 0.050 & -3.0 & 30.0 \\
\hline Chloroform & 0.500 & 0.499 & 0.200 & -0.2 & 30.0 \\
\hline 1,1,1-Trichloroethane & 0.625 & 0.639 & 0.100 & 2.2 & 30.0 \\
\hline Cyclohexane & 0.890 & 0.922 & 0.010 & 3.6 & 40.0 \\
\hline Carbon tetrachloride & 0.522 & 0.536 & 0.100 & 2.7 & 30.0 \\
\hline Benzene & 1.820 & 1.834 & 0.400 & 0.8 & 30.0 \\
\hline 1,2-Dichloroethane & 0.206 & 0.201 & 0.100 & -2.3 & 30.0 \\
\hline Trichloroethene & 0.427 & 0.432 & 0.300 & 1.3 & 30.0 \\
\hline Methylcyclohexane & 0.679 & 0.706 & 0.010 & 3.9 & 40.0 \\
\hline
\end{tabular}

Report 1,4-Dioxane for Low-Medium VOA analysis only 
7B - FORM VII VOA-2

VOLATILE CONTINUING CALIBRATION DATA

Lab Name: TESTAMERICA BURLINGTON

Iuab Code: STIV Case No.: CNTRALIA Mod. Ref No.:
Contract: 8E-00302

Calibration Date: 04/09/2010 Time: 0806

Init. Calib. Date(s): 04/05/2010 04/05/2010

EPA Sample No. (VSTD\#\#\#\#\#): VSTD005JN Init. Calib. Time(s): 13181505

Heated Purge: (Y/N)N GC Column: DB-624 ID: 0.53 (mm) Iength: 75 (m)

Purge Volume: 25.0

(mL)

\begin{tabular}{|c|c|c|c|c|c|}
\hline COMPOUND & $\overline{\mathrm{RRF}}$ & RRF5. 0 & $\begin{array}{l}\text { MIN } \\
\text { RRF }\end{array}$ & $\div D$ & MAX $\div D$ \\
\hline \multicolumn{6}{|c|}{ 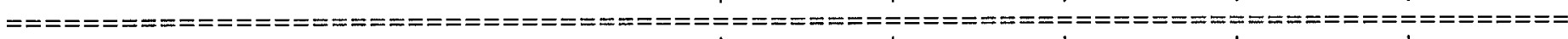 } \\
\hline 1,2-Dichloropropane & 0.373 & 0.372 & 0.010 & -0.3 & 40.0 \\
\hline Bromodichloromethane & 0.386 & 0.380 & 0.200 & -1.6 & 30.0 \\
\hline cis-1,3-Dichloropropene & 0.500 & 0.495 & 0.200 & -0.9 & 30.0 \\
\hline 4-Methyl-2-pentanone & 0.096 & 0.089 & 0.010 & -7.8 & 40.0 \\
\hline Toluene & 1.916 & 1.929 & 0.400 & 0.7 & 30.0 \\
\hline trans-1,3-Dichloropropene & 0.343 & 0.338 & 0.100 & -1.4 & 30.0 \\
\hline $1,1,2$-Trichloroethane & 0.156 & 0.149 & 0.100 & -4.3 & 30.0 \\
\hline Tetrachloroethene & 0.340 & 0.345 & 0.100 & 1.7 & 30.0 \\
\hline 2-Hexanone & 0.061 & 0.056 & 0.010 & -8.6 & 40.0 \\
\hline Dibromochloromethane & 0.182 & 0.175 & 0.100 & -3.7 & 30.0 \\
\hline 1,2-Dibromoethane & 0.137 & 0.131 & 0.010 & -4.0 & 30.0 \\
\hline Chlorobenzene & 1.014 & 0.992 & 0.500 & -2.2 & 30.0 \\
\hline Ethylbenzene & 2.128 & 2.180 & 0.100 & 2.5 & 30.0 \\
\hline o-Xylene & 0.730 & 0.721 & 0.300 & $-1 \cdot 2$ & 30.0 \\
\hline $\mathrm{m}, \mathrm{p}$-xylene & 0.793 & 0.821 & 0.300 & 3.5 & 30.0 \\
\hline Styrene & 1.070 & 1.071 & 0.300 & 0.2 & 30.0 \\
\hline Bromoform & 0.191 & 0.192 & 0.050 & 0.4 & 30.0 \\
\hline Isopropylbenzene & 2.040 & 2.103 & 0.010 & 3.1 & 40.0 \\
\hline $1,1,2,2$-Tetrachloroethane & 0.147 & 0.137 & 0.100 & -6.7 & 30.0 \\
\hline 1,3-Dichlorobenzene & 1.634 & 1.607 & 0.400 & -1.6 & 30.0 \\
\hline 1,4-Dichlorobenzene & 1.560 & 1.544 & 0.400 & $-1 \cdot 1$ & 30.0 \\
\hline 1,2-Dichlorobenzene & 1.209 & 1.182 & 0.400 & -2.2 & 30.0 \\
\hline 1,2-Dibromo-3-chloropropane & 0.050 & 0.042 & 0.010 & $-15 \cdot 9$ & 40.0 \\
\hline $1,2,4$-Trichlorobenzene & 0.553 & 0.535 & 0.200 & -3.3 & 30.0 \\
\hline 1,2,3-Trichlorobenzene & 0.369 & 0.357 & 0.200 & $-3 \cdot 3$ & 30.0 \\
\hline
\end{tabular}


$7 C$ - FORM VII VOA-3

VOLATILE CONTINUING CALIBRATION DATA

Lab Name: TESTAMERICA BURLINGTON

Contract: $8 \mathrm{E}-00302$

Lab Code: STLV Case No.: CNTRALIA Mod. Ref No.:

SDG No.: 136697

Instrument ID: J.i

Calibration Date: 04/09/2010 Time: 0806

Lab File ID: JAQ002FV

Init. Calib. Date(s) : 04/05/2010 04/05/2010

EPA Sample No. (VSTD\#\#\#\#): VSTD005JN

Init. Calib. Time(s): $1318 \quad 1505$

Heated Purge: $(\mathrm{Y} / \mathrm{N}) \mathrm{N}$

GC Column: DB-624 ID: 0.53

(mm) Length: 75

(m)

Purge Volume: 25.0

(mL)

\begin{tabular}{|c|c|c|c|c|c|}
\hline COMPOUND & $\overline{\mathrm{RRF}}$ & RRF5. 0 & $\begin{array}{l}\text { MIN } \\
\text { RRF }\end{array}$ & $\div \mathrm{D}$ & $\mathrm{MAX} \div \mathrm{D}$ \\
\hline \multirow{2}{*}{\multicolumn{6}{|c|}{ 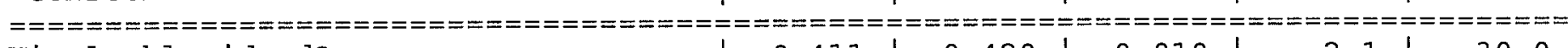 }} \\
\hline Vinyl chloride-d3 & 0.411 & 0.420 & 0.010 & & 30.0 \\
\hline Chloroethane-d5 & 0.310 & 0.322 & 0.010 & 3.8 & 40.0 \\
\hline 1,1-Dichloroethene-d2 & 0.683 & 0.700 & 0.010 & 2.5 & 30.0 \\
\hline 2-Butanone-d5 & 0.027 & 0.023 & 0.010 & -13.0 & 40.0 \\
\hline Chloroform-d & 0.541 & 0.530 & 0.010 & -1.9 & 30.0 \\
\hline 1,2-Dichloroethane-d4 & 0.169 & 0.166 & 0.010 & -2.0 & 30.0 \\
\hline Benzene-d6 & 1.760 & 1.759 & 0.400 & -0.0 & 30.0 \\
\hline 1,2-Dichloropropane-d6 & 0.499 & 0.441 & 0.010 & -11.6 & 40.0 \\
\hline Toluene-d8 & 1.583 & 1.619 & 0.010 & 2.2 & 30.0 \\
\hline trans-1,3-Dichloropropene-d4 & 0.312 & 0.302 & 0.010 & -3.1 & 30.0 \\
\hline 2 -Hexanone-d5 & 0.033 & 0.028 & 0.010 & -15.5 & 40.0 \\
\hline $1,1,2,2$-Tetrachloroethane-d2 & 0.151 & 0.137 & 0.010 & -9.2 & 30.0 \\
\hline 1,2-Dichlorobenzene-d4 & 0.764 & 0.760 & 0.010 & -0.6 & 30.0 \\
\hline
\end{tabular}

Report 1,4-Dioxane-d8 for Low-Medium VOA analysis only 
$7 A$ - FORM VII VOA-1

VOLATILE CONTINUING CALIBRATION DATA

Lab Name: TESTAMERICA BURLINGTON

Contract: $8 \mathrm{E}-00302$

Lab Code: STLV Case No.: CNTRALIA Mod. Ref No.:

SDG No.: 136697

Instrument ID: J.i

Calibration Date: 04/09/2010 Time: 1740

Lab File ID: JAQ05FC1

Init. Calib. Date(s): 04/05/2010 04/05/2010

EPA Sample No. (VSTD\#\#\#\#): VSTD005NJ Init. Calib. Time(s): $1318 \quad 1505$

Heated Purge: ( $\mathrm{Y} / \mathrm{N}) \mathrm{N}$ GC Column: DB-624 ID: 0.53 (mm) Length: 75

$(\mathrm{m})$

Purge Volume: 25.0

$(\mathrm{mL})$

\begin{tabular}{|c|c|c|c|c|c|}
\hline COMPOUND & $\overline{\mathrm{RRF}}$ & RRF5. 0 & $\begin{array}{l}\text { MIN } \\
\text { RRF }\end{array}$ & $\because \mathrm{D}$ & MAX $\div D$ \\
\hline \multicolumn{6}{|c|}{ 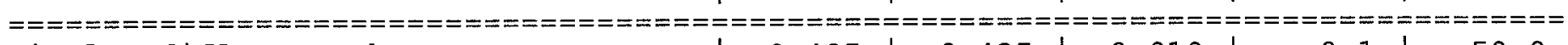 } \\
\hline Dichlorodifluoromethane & 0.425 & 0.425 & 0.010 & 0.1 & 50.0 \\
\hline Chloromethane & 0.451 & 0.482 & 0.010 & 7.0 & 50.0 \\
\hline Vinyl chloride & 0.454 & 0.468 & 0.010 & 3.0 & 50.0 \\
\hline Bromomethane & 0.171 & 0.166 & 0.010 & -3.3 & 50.0 \\
\hline Chloroethane & 0.263 & 0.268 & 0.010 & 1.9 & 50.0 \\
\hline Trichlorofluoromethane & 0.511 & 0.510 & 0.010 & -0.3 & 50.0 \\
\hline 1,1-Dichloroethene & 0.306 & 0.299 & 0.010 & -2.2 & 50.0 \\
\hline $1,1,2$-Trichloro-1,2,2-trifluoroethane & 0.323 & 0.329 & 0.010 & 1.7 & 50.0 \\
\hline Acetone & 0.014 & 0.012 & 0.010 & -15.4 & 50.0 \\
\hline Carbon disulfide & 0.986 & 0.956 & 0.010 & -3.1 & 50.0 \\
\hline Methyl acetate & 0.053 & 0.048 & 0.010 & -9.4 & 50.0 \\
\hline Methylene chloride & 0.250 & 0.248 & 0.010 & -1.1 & 50.0 \\
\hline trans-1,2-Dichloroethene & 0.326 & 0.322 & 0.010 & -1.0 & 50.0 \\
\hline Methyl tert-butyl ether & 0.397 & 0.368 & 0.010 & -7.2 & 50.0 \\
\hline 1,1 -Dichloroethane & 0.597 & 0.593 & 0.010 & -0.6 & 50.0 \\
\hline cis-1,2-Dichloroethene & 0.316 & 0.308 & 0.010 & -2.5 & 50.0 \\
\hline 2-Butanone & 0.028 & 0.026 & 0.010 & -6.4 & 50.0 \\
\hline Bromochloromethane & 0.090 & 0.086 & 0.010 & -4.4 & 50.0 \\
\hline Chloroform & 0.500 & 0.498 & 0.010 & -0.6 & 50.0 \\
\hline $1,1,1$-Trichloroethane & 0.625 & 0.631 & 0.010 & 1.0 & 50.0 \\
\hline Cyclohexane & 0.890 & 0.894 & 0.010 & 0.5 & 50.0 \\
\hline Carbon tetrachloride & 0.522 & 0.528 & 0.010 & 1.1 & 50.0 \\
\hline Benzene & 1.820 & 1.817 & 0.010 & -0.2 & 50.0 \\
\hline 1,2-Dichloroethane & 0.206 & 0.203 & 0.010 & -1.3 & 50.0 \\
\hline Trichloroethene & 0.427 & 0.430 & 0.010 & 0.7 & 50.0 \\
\hline Methylcyclohexane & 0.679 & 0.700 & 0.010 & 3.1 & 50.0 \\
\hline
\end{tabular}

Report 1,4-Dioxane for Low-Medium VOA analysis only

SOMO1.2 
7B - FORM VII VOA-2

VOLATILE CONTINUING CALIBRATION DATA

Lab Name: TESTAMERICA BURIINGTON

Contract: $8 \mathrm{E}-00302$

Lab Code: STLV Case No.: CNTRALIA Mod. Ref No.:

SDG NO.: 136697

Instrument ID: J.i

Calibration Date: 04/09/2010 Time: 1740

Lab File ID: JAQ05FCI

Init. Calib. Date(s): 04/05/2010 04/05/2010

EPA Sample No. (VSTD\#\#\#\#\#): VSTD005NJ Init. Calib. Time(s): $1318 \quad 1505$

Heated Purge: (Y/N)N GC Column: DB-624 ID: $0.53 \quad$ (mm) Length: $75 \quad$ (m)

Purge Volume: 25.0

$(\mathrm{mL})$

\begin{tabular}{|c|c|c|c|c|c|}
\hline COMPOUND & $\overline{\mathrm{RRF}}$ & RRF5.0 & $\begin{array}{l}\text { MIN } \\
\text { RRF }\end{array}$ & $\div \mathrm{D}$ & MAX $\div D$ \\
\hline \multicolumn{6}{|c|}{ 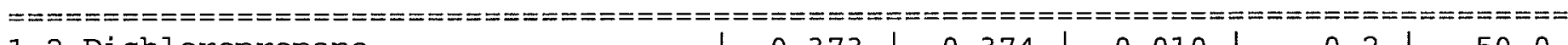 } \\
\hline 1,2-Dichloropropane & 0.373 & 0.374 & 0.010 & 0.2 & 50.0 \\
\hline Bromodichloromethane & 0.386 & 0.380 & 0.010 & -1.7 & 50.0 \\
\hline cis-1,3-Dichloropropene & 0.500 & 0.493 & 0.010 & -1.3 & 50.0 \\
\hline 4-Methyl-2-pentanone & 0.096 & 0.092 & 0.010 & -4.3 & 50.0 \\
\hline Toluene & 1.916 & 1.902 & 0.010 & -0.7 & 50.0 \\
\hline trans-1,3-Dichloropropene & 0.343 & 0.342 & 0.010 & -0.3 & 50.0 \\
\hline 1,1,2-Trichloroethane & 0.156 & 0.151 & 0.010 & -3.1 & 50.0 \\
\hline Tetrachloroethene & 0.340 & 0.347 & 0.010 & 2.1 & 50.0 \\
\hline 2-Hexanone & 0.061 & 0.059 & 0.010 & -3.1 & 50.0 \\
\hline Dibromochloromethane & 0.182 & 0.181 & 0.010 & -0.4 & 50.0 \\
\hline 1,2-Dibromoethane & 0.137 & 0.132 & 0.010 & -3.5 & 50.0 \\
\hline Chlorobenzene & 1.014 & 1.019 & 0.010 & 0.6 & 50.0 \\
\hline Ethylbenzene & 2.128 & 2.131 & 0.010 & 0.2 & 50.0 \\
\hline o-XYlene & 0.730 & 0.724 & 0.010 & -0.8 & 50.0 \\
\hline $\mathrm{m}, \mathrm{p}-\mathrm{xy}$ lene & 0.793 & 0.800 & 0.010 & 0.9 & 50.0 \\
\hline styrene & 1.070 & 1.064 & 0.010 & -0.5 & 50.0 \\
\hline Bromoform & 0.191 & 0.180 & 0.010 & -5.6 & 50.0 \\
\hline Isopropylbenzene & 2.040 & 2.085 & 0.010 & 2.2 & 50.0 \\
\hline $1,1,2,2$-Tetrachloroethane & 0.147 & 0.144 & 0.010 & -1.7 & 50.0 \\
\hline 1,3-Dichlorobenzene & 1.634 & 1.571 & 0.010 & -3.8 & 50.0 \\
\hline 1,4-Dichlorobenzene & 1.560 & 1.508 & 0.010 & -3.4 & 50.0 \\
\hline 1,2-Dichlorobenzene & 1.209 & 1.154 & 0.010 & -4.5 & 50.0 \\
\hline 1,2-Dibromo-3-chloropropane & 0.050 & 0.040 & 0.010 & -19.3 & 50.0 \\
\hline $1,2,4$-Trichlorobenzene & 0.553 & 0.538 & 0.010 & -2.6 & 50.0 \\
\hline 1,2,3-Trichlorobenzene & 0.369 & 0.383 & 0.010 & 3.7 & 50.0 \\
\hline
\end{tabular}

SOM01.2 
$7 C$ - FORM VII VOA-3

VOLATILE CONTINUING CALIBRATION DATA

Lab Name: TESTAMERICA BURLINGTON

Contract: $8 \mathrm{E}-00302$

Lab Code: STLV Case No.: CNTRALIA Mod. Ref No.:

SDG NO.: 136697

Instrument ID: J.i

Calibration Date: 04/09/2010 Time: 1740

Lab File ID: JAQ05FC1

Init. Calib. Date(s): 04/05/2010 04/05/2010

EPA Sample No. (VSTD\#\#\#\#): VSTD005NJ

Init. Calib. Time(s): $1318 \quad 1505$

Heated Purge: (Y/N)N GC Column: DB-624 ID: $0.53 \quad$ (mm) Length: 75 (m)

Purge Volume: 25.0

(mL)

\begin{tabular}{|c|c|c|c|c|c|}
\hline COMPOUND & $\overline{\mathrm{RRF}}$ & RRF5. 0 & $\begin{array}{l}\text { MIN } \\
\text { RRF }\end{array}$ & $\% \mathrm{D}$ & MAX $\div D$ \\
\hline \multirow{2}{*}{\multicolumn{6}{|c|}{ 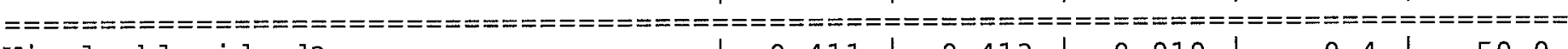 }} \\
\hline Vinyl chloride-d3 & 0.411 & 0.413 & 0.010 & & 50.0 \\
\hline Chloroethane-d5 & 0.310 & 0.322 & 0.010 & 3.8 & 50.0 \\
\hline 1,1-Dichloroethene- $\mathrm{d} 2$ & 0.683 & 0.686 & 0.010 & 0.5 & 50.0 \\
\hline 2-Butanone-d5 & 0.027 & 0.026 & 0.010 & -4.8 & 50.0 \\
\hline Chloroform-d & 0.541 & 0.533 & 0.010 & -1.3 & 50.0 \\
\hline $1,2-$ Dichloroet hane- $\mathrm{d} 4$ & 0.169 & 0.167 & 0.010 & -1.4 & 50.0 \\
\hline Benzene-d6 & 1.760 & 1.744 & 0.010 & -0.9 & 50.0 \\
\hline 1,2-Dichloropropane-d6 & 0.499 & 0.494 & 0.010 & -1.1 & 50.0 \\
\hline Toluene-d8 & 1.583 & 1.598 & 0.010 & 0.9 & 50.0 \\
\hline trans-1,3-Dichloropropene-d4 & 0.312 & 0.308 & 0.010 & -1.3 & 50.0 \\
\hline 2 -Hexanone-d5 & 0.033 & 0.030 & 0.010 & -11.3 & 50.0 \\
\hline $1,1,2,2$-Tetrachloroethane-d2 & 0.151 & 0.140 & 0.010 & -7.9 & 50.0 \\
\hline 1,2-Dichlorobenzene-d4 & 0.764 & 0.748 & 0.010 & -2.1 & 50.0 \\
\hline
\end{tabular}

Report 1,4-Dioxane-d8 for Low-Medium VOA analysis only 
7A - FORM VII VOA-1

VOLATILE CONTINUING CALIBRATION DATA

Lab Name: TESTAMERICA BURLINGTON

Contract: $8 \mathrm{E}-00302$

Lab Code: STLV Case No.: CNTRALIA Mod. Ref No.:

SDG NO.: 136697

Instrument ID: J.i

Calibration Date: 04/10/2010 Time: 1015

Lab File ID: JAQ005GV

Init. Calib. Date(s): 04/05/2010 04/05/2010

EPA Sample No. (VSTD\#\#\#\#): VSTD005Jo Init. Calib. Time(s): 13181505

Heated Purge: $(\mathrm{Y} / \mathrm{N}) \mathrm{N}$ GC Column: DB-624 ID: $0.53 \quad$ (mm) Length: $75 \quad$ (m)

Purge Volume: 25.0

$(\mathrm{mL})$

\begin{tabular}{|c|c|c|c|c|c|}
\hline COMPOUND & $\overline{\mathrm{RRF}}$ & RRF5. 0 & $\begin{array}{l}\text { MIN } \\
\text { RRF }\end{array}$ & $\div \mathrm{D}$ & MAX $\div \mathrm{D}$ \\
\hline \multicolumn{6}{|c|}{ 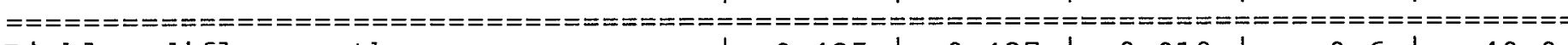 } \\
\hline Dichlorodifluoromethane & 0.425 & 0.427 & 0.010 & 0.6 & 40.0 \\
\hline Chloromethane & 0.451 & 0.479 & 0.010 & 6.4 & 40.0 \\
\hline Vinyl chloride & 0.454 & 0.477 & 0.100 & 4.9 & 30.0 \\
\hline Bromomethane & 0.171 & 0.185 & 0.100 & 7.8 & 30.0 \\
\hline Chloroethane & 0.263 & 0.268 & 0.010 & 2.0 & 40.0 \\
\hline Trichlorofluoromethane & 0.511 & 0.523 & 0.010 & 2.2 & 40.0 \\
\hline 1,1-Dichloroethene & 0.306 & 0.305 & 0.100 & -0.2 & 30.0 \\
\hline 1,1,2-Trichloro-1,2,2-trifluoroethane & 0.323 & 0.333 & 0.010 & 2.9 & 40.0 \\
\hline Acetone & 0.014 & 0.012 & 0.010 & -15.6 & 40.0 \\
\hline Carbon disulfide & 0.986 & 0.977 & 0.010 & -1.0 & 40.0 \\
\hline Methyl acetate & 0.053 & 0.046 & 0.010 & -12.3 & 40.0 \\
\hline Methylene chloride & 0.250 & 0.240 & 0.010 & -4.2 & 40.0 \\
\hline trans-1,2-Dichloroethene & 0.326 & 0.333 & 0.010 & 2.4 & 40.0 \\
\hline Methyl tert-butyl ether & 0.397 & 0.356 & 0.010 & -10.2 & 40.0 \\
\hline 1,1-Dichloroethane & 0.597 & 0.611 & 0.200 & 2.4 & 30.0 \\
\hline cis-1,2-Dichloroethene & 0.316 & 0.313 & 0.010 & -0.8 & 40.0 \\
\hline 2-Butanone & 0.028 & 0.024 & 0.010 & -13.6 & 40.0 \\
\hline Bromochloromethane & 0.090 & 0.087 & 0.050 & -2.4 & 30.0 \\
\hline Chloroform & 0.500 & 0.509 & 0.200 & 1.7 & 30.0 \\
\hline 1,1,1-Trichloroethane & 0.625 & 0.644 & 0.100 & 3.1 & 30.0 \\
\hline Cyclohexane & 0.890 & 0.933 & 0.010 & 4.8 & 40.0 \\
\hline Carbon tetrachloride & 0.522 & 0.543 & 0.100 & 4.1 & 30.0 \\
\hline Benzene & 1.820 & 1.838 & 0.400 & 1.0 & 30.0 \\
\hline 1,2-Dichloroethane & 0.206 & 0.203 & 0.100 & -1.3 & 30.0 \\
\hline Trichloroethene & 0.427 & 0.442 & 0.300 & 3.6 & 30.0 \\
\hline Methylcyclohexane & 0.679 & 0.716 & 0.010 & 5.4 & 40.0 \\
\hline
\end{tabular}

Report 1,4-Dioxane for Low-Medium VOA analysis only

SOMO1.2 
$7 B$ - FORM VII VOA-2

VOLATILE CONTINUING CALIBRATION DATA

Lab Name: TESTAMERICA BURLINGTON

Lab Code: STLV Case No.: CNTRALIA Mod. Ref No.:
Contract : $8 \mathrm{E}-00302$
SDG No.: 136697
Instrument ID: J.i

Lab File ID: JAQ005GV

EPA Sample No. (VSTD\#\#\#\#\#): VSTD005JO
Calibration Date: 04/10/2010 Time: 1015

Init. Calib. Date(s): 04/05/2010 04/05/2010

Init. Calib. Time(s): $1318 \quad 1505$

Heated Purge: (Y/N)N GC Column: DB-624 ID: $0.53 \quad$ (mm) Length: $75 \quad$ (m)

Purge Volume: 25.0

$(\mathrm{mL})$

\begin{tabular}{|c|c|c|c|c|c|}
\hline COMPOUND & $\overline{\mathrm{RRF}}$ & RRF5. 0 & $\begin{array}{l}\text { MIN } \\
\text { RRF }\end{array}$ & $\div \mathrm{D}$ & MAX $\div D$ \\
\hline \multicolumn{6}{|c|}{ 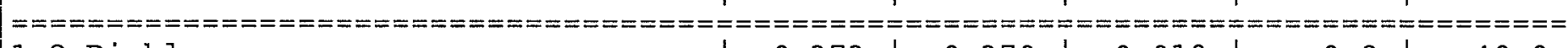 } \\
\hline 1,2-Dichloropropane & 0.373 & 0.370 & 0.010 & -0.8 & 40.0 \\
\hline Bromodichloromethane & 0.386 & 0.380 & 0.200 & -1.6 & 30.0 \\
\hline cis-1,3-Dichloropropene & 0.500 & 0.497 & 0.200 & -0.6 & 30.0 \\
\hline 4-Methyl-2-pentanone & 0.096 & 0.089 & 0.010 & -7.2 & 40.0 \\
\hline Toluene & 1.916 & 1.932 & 0.400 & 0.9 & 30.0 \\
\hline trans-1,3-Dichloropropene & 0.343 & 0.336 & 0.100 & -1.9 & 30.0 \\
\hline 1,1,2-Trichloroethane & 0.156 & 0.152 & 0.100 & -2.7 & 30.0 \\
\hline Tetrachloroethene & 0.340 & 0.361 & 0.100 & 6.3 & 30.0 \\
\hline 2-Hexanone & 0.061 & 0.057 & 0.010 & -6.7 & 40.0 \\
\hline Dibromochloromethane & 0.182 & 0.180 & 0.100 & -1.0 & 30.0 \\
\hline 1,2-Dibromoethane & 0.137 & 0.132 & 0.010 & -3.7 & 30.0 \\
\hline Chlorobenzene & 1.014 & 1.013 & 0.500 & -0.0 & 30.0 \\
\hline Ethylbenzene & 2.128 & 2.168 & 0.100 & 1.9 & 30.0 \\
\hline o-xylene & 0.730 & 0.731 & 0.300 & 0.2 & 30.0 \\
\hline $\mathrm{m}, \mathrm{p}$-xylene & 0.793 & 0.817 & 0.300 & 3.0 & 30.0 \\
\hline styrene & 1.070 & 1.077 & 0.300 & 0.7 & 30.0 \\
\hline Bromoform & 0.191 & 0.186 & 0.050 & -2.6 & 30.0 \\
\hline Isopropylbenzene & 2.040 & 2.122 & 0.010 & 4.0 & 40.0 \\
\hline $1,1,2,2$-Tetrachloroethane & 0.147 & 0.132 & 0.100 & -9.9 & 30.0 \\
\hline 1,3-Dichlorobenzene & 1.634 & 1.618 & 0.400 & -0.9 & 30.0 \\
\hline 1,4-Dichlorobenzene & 1.560 & 1.531 & 0.400 & -1.9 & 30.0 \\
\hline 1,2-Dichlorobenzene & 1.209 & 1.162 & 0.400 & -3.9 & 30.0 \\
\hline 1,2-Dibromo-3-chloropropane & 0.050 & 0.040 & 0.010 & -19.5 & 40.0 \\
\hline $1,2,4$-Trichlorobenzene & 0.553 & 0.525 & 0.200 & -5.0 & 30.0 \\
\hline 1,2,3-Trichlorobenzene & 0.369 & 0.346 & 0.200 & -6.4 & 30.0 \\
\hline
\end{tabular}


7C - FORM VII VOA-3

VOLATILE CONTINUING CALIBRATION DATA

Lab Name: TESTAMERICA BURLINGTON

Contract: $8 \mathrm{E}-00302$

Lab Code: STLV Case No.: CNTRALIA Mod. Ref No.:

SDG No.: 136697

Instrument ID: J.i

Calibration Date: 04/10/2010 Time: 1015

Lab File ID: JAQ005GV

Init. Calib. Date(s): 04/05/2010 04/05/2010

EPA Sample No. (VSTD\#\#\#\#): VSTD005JO

Init. Calib. Time(s): $1318 \quad 1505$

Heated Purge: (Y/N)N GC Column: DB-624

ID $: 0.53$

(mm) Length: 75

(m)

Purge Volume: 25.0

$(\mathrm{mL})$

\begin{tabular}{|c|c|c|c|c|c|}
\hline COMPOUND & $\overline{\mathrm{RRF}}$ & RRF5.0 & $\begin{array}{l}\text { MIN } \\
\text { RRF }\end{array}$ & $\because \mathrm{D}$ & $\operatorname{MAX} \div \mathrm{D}$ \\
\hline \multirow{2}{*}{\multicolumn{6}{|c|}{ 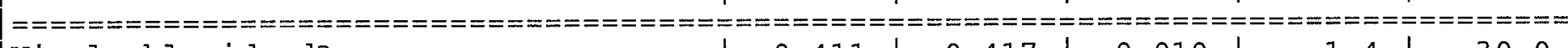 }} \\
\hline Vinyl chloride-d3 & 0.411 & 0.417 & 0.010 & & \\
\hline Chloroethane-d5 & 0.310 & 0.325 & 0.010 & 4.9 & 40.0 \\
\hline 1,1 -Dichloroethene-d2 & 0.683 & 0.704 & 0.010 & 3.1 & 30.0 \\
\hline 2-Butanone-d5 & 0.027 & 0.023 & 0.010 & -14.0 & 40.0 \\
\hline Chloroform-d & 0.541 & 0.533 & 0.010 & -1.5 & 30.0 \\
\hline 1,2-Dichloroethane-d4 & 0.169 & 0.160 & 0.010 & -5.1 & 30.0 \\
\hline Benzene-d 6 & 1.760 & 1.784 & 0.400 & 1.4 & 30.0 \\
\hline 1,2-Dichloropropane-d6 & 0.499 & 0.440 & 0.010 & -11.8 & 40.0 \\
\hline Toluene-d8 & 1.583 & 1.623 & 0.010 & 2.5 & 30.0 \\
\hline trans-1,3-Dichloropropene-d4 & 0.312 & 0.304 & 0.010 & -2.4 & 30.0 \\
\hline 2 -Hexanone-d5 & 0.033 & 0.028 & 0.010 & -16.9 & 40.0 \\
\hline $1,1,2,2$-Tetrachloroethane-d2 & 0.151 & 0.137 & 0.010 & -9.5 & 30.0 \\
\hline 1,2-Dichlorobenzene-d4 & 0.764 & 0.755 & 0.010 & -1.1 & 30.0 \\
\hline
\end{tabular}

Report 1,4-Dioxane-d8 for Low-Medium VOA analysis only 
$7 A$ - FORM VII VOA-1

VOLATILE CONTINUING CALIBRATION DATA

Lab Name: TESTAMERICA BURLINGTON

Contract: $8 \mathrm{E}-00302$

Lab Code: STLV Case No.: CNTRALIA Mod. Ref No.:

SDG No.: 136697

Instrument ID: J.i

Calibration Date: 04/10/2010 Time: 1925

Lab File ID: JAQ05GC1

Init. Calib. Date(s): 04/05/2010 04/05/2010

EPA Sample No. (VSTD\#\#\#\#) : VSTD0050J Init. Calib. Time(s): 13181505

Heated Purge: ( $\mathrm{Y} / \mathrm{N}) \mathrm{N}$ GC Column: DB-624 ID: $0.53 \quad$ (mm) Length: 75 (m)

Purge Volume: 25.0

(mL)

\begin{tabular}{|c|c|c|c|c|c|}
\hline COMPOUND & $\overline{\mathrm{RRF}}$ & RRF5. 0 & $\begin{array}{l}\text { MIN } \\
\text { RRF }\end{array}$ & $\because \mathrm{D}$ & $\mathrm{MAX} \div \mathrm{D}$ \\
\hline \multicolumn{6}{|c|}{ 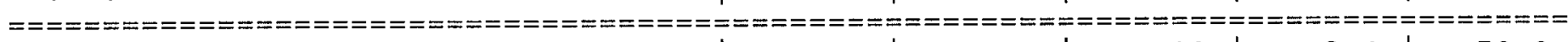 } \\
\hline Dichlorodifluoromethane & 0.425 & 0.414 & 0.010 & -2.4 & 50.0 \\
\hline Chloromethane & 0.451 & 0.491 & 0.010 & 9.0 & 50.0 \\
\hline vinyl chloride & 0.454 & 0.479 & 0.010 & 5.4 & 50.0 \\
\hline Bromomethane & 0.171 & 0.177 & 0.010 & 3.2 & 50.0 \\
\hline Chloroethane & 0.263 & 0.271 & 0.010 & 3.1 & 50.0 \\
\hline Trichlorofluoromethane & 0.511 & 0.516 & 0.010 & 1.0 & 50.0 \\
\hline 1,1-Dichloroethene & 0.306 & 0.312 & 0.010 & 2.0 & 50.0 \\
\hline 1,1,2-Trichloro-1,2,2-trifluoroethane & 0.323 & 0.328 & 0.010 & 1.5 & 50.0 \\
\hline Acetone & 0.014 & 0.013 & 0.010 & -11.0 & 50.0 \\
\hline Carbon disulfide & 0.986 & 0.952 & 0.010 & -3.4 & 50.0 \\
\hline Methyl acetate & 0.053 & 0.045 & 0.010 & -14.5 & 50.0 \\
\hline Methylene chloride & 0.250 & 0.247 & 0.010 & -1.2 & 50.0 \\
\hline trans-1,2-Dichloroethene & 0.326 & 0.327 & 0.010 & 0.4 & 50.0 \\
\hline Methyl tert-butyl ether & 0.397 & 0.353 & 0.010 & -11.0 & 50.0 \\
\hline 1,1-Dichloroethane & 0.597 & 0.612 & 0.010 & 2.6 & 50.0 \\
\hline cis-1,2-Dichloroethene & 0.316 & 0.310 & 0.010 & -1.7 & 50.0 \\
\hline 2-Butanone & 0.028 & 0.025 & 0.010 & $-10 \cdot 1$ & 50.0 \\
\hline Bromochloromethane & 0.090 & 0.086 & 0.010 & $-3 \cdot 4$ & 50.0 \\
\hline Chloroform & 0.500 & 0.509 & 0.010 & 1.8 & 50.0 \\
\hline 1,1,1-Trichloroethane & 0.625 & 0.654 & 0.010 & 4.7 & 50.0 \\
\hline Cyclohexane & 0.890 & 0.936 & 0.010 & 5.1 & 50.0 \\
\hline Carbon tetrachloride & 0.522 & 0.549 & 0.010 & 5.2 & 50.0 \\
\hline Benzene & 1.820 & 1.855 & 0.010 & 1.9 & 50.0 \\
\hline 1,2-Dichloroethane & 0.206 & 0.201 & 0.010 & -2.1 & 50.0 \\
\hline Trichloroethene & 0.427 & 0.447 & 0.010 & 4.9 & 50.0 \\
\hline Methyl cyclohexane & 0.679 & 0.725 & 0.010 & 6.8 & 50.0 \\
\hline
\end{tabular}

Report 1,4-Dioxane for Low-Medium VOA analysis only 
7B - FORM VII VOA-2

VOLATILE CONTINUING CALIBRATION DATA

Lab Name: TESTAMERICA BURLINGTON

Contract: $8 \mathrm{E}-00302$

Lab Code: STLV Case No.: CNTRALIA Mod. Ref No.:

SDG No.: 136697

Instrument ID: J.i

Calibration Date: 04/10/2010 Time: 1925

Lab File ID: JAQ05GC1

Init. Calib. Date(s) : 04/05/2010 04/05/2010

EPA Sample No. (VSTD\#\#\#\#\#): VSTD0050J

Init. Calib. Time(s) : $1318 \quad 1505$

Heated Purge: ( $\mathrm{Y} / \mathrm{N}) \mathrm{N}$ GC Column: DB-624 ID: $0.53 \quad$ (mm) Length: 75

(m)

Purge Volume: 25.0

(mL)

\begin{tabular}{|c|c|c|c|c|c|}
\hline COMPOUND & $\overline{\mathrm{RRF}}$ & RRF5. 0 & $\begin{array}{l}\text { MIN } \\
\text { RRF }\end{array}$ & $\because \mathrm{D}$ & MAX $\div \mathrm{D}$ \\
\hline \multicolumn{6}{|c|}{ =" } \\
\hline 1,2-Dichloropropane & 0.373 & 0.375 & 0.010 & 0.4 & 50.0 \\
\hline Bromodichloromethane & 0.386 & 0.380 & 0.010 & -1.5 & 50.0 \\
\hline cis-1,3-Dichloropropene & 0.500 & 0.501 & 0.010 & 0.3 & 50.0 \\
\hline 4-Methyl-2-pentanone & 0.096 & 0.092 & 0.010 & -4.5 & 50.0 \\
\hline Toluene & 1.916 & 1.951 & 0.010 & 1.9 & 50.0 \\
\hline trans-1,3-Dichloropropene & 0.343 & 0.336 & 0.010 & -2.0 & 50.0 \\
\hline 1,1,2-Trichloroethane & 0.156 & 0.154 & 0.010 & -1.6 & 50.0 \\
\hline Tetrachloroethene & 0.340 & 0.352 & 0.010 & 3.6 & 50.0 \\
\hline 2-Hexanone & 0.061 & 0.058 & 0.010 & -4.7 & 50.0 \\
\hline Dibromochloromethane & 0.182 & 0.178 & 0.010 & -2.1 & 50.0 \\
\hline 1,2-Dibromoethane & 0.137 & 0.130 & 0.010 & -4.8 & 50.0 \\
\hline Chlorobenzene & 1.014 & 1.028 & 0.010 & 1.4 & 50.0 \\
\hline Ethylbenzene & 2.128 & 2.182 & 0.010 & 2.6 & 50.0 \\
\hline o-xylene & 0.730 & 0.729 & 0.010 & -0.1 & 50.0 \\
\hline $\mathrm{m}, \mathrm{p}$-xylene & 0.793 & 0.814 & 0.010 & 2.7 & 50.0 \\
\hline Styrene & 1.070 & 1.089 & 0.010 & 1.8 & 50.0 \\
\hline Bromoform & 0.191 & 0.184 & 0.010 & -3.6 & 50.0 \\
\hline Isopropylbenzene & 2.040 & 2.118 & 0.010 & 3.8 & 50.0 \\
\hline $1,1,2,2$-Tetrachloroethane & 0.147 & 0.135 & 0.010 & -8.0 & 50.0 \\
\hline 1,3-Dichlorobenzene & 1.634 & 1.612 & 0.010 & -1.3 & 50.0 \\
\hline 1,4-Dichlorobenzene & 1.560 & 1.531 & 0.010 & -1.9 & 50.0 \\
\hline 1,2-Dichlorobenzene & 1.209 & 1.157 & 0.010 & -4.3 & 50.0 \\
\hline 1,2-Dibromo-3-chloropropane & 0.050 & 0.044 & 0.010 & -11.6 & 50.0 \\
\hline 1,2,4-Trichlorobenzene & 0.553 & 0.533 & 0.010 & -3.6 & 50.0 \\
\hline 1,2,3-Trichlorobenzene & 0.369 & 0.359 & 0.010 & -2.6 & 50.0 \\
\hline
\end{tabular}

SOMO 1.2 


$$
7 C \text { - FORM VII VOA-3 }
$$

VOLATILE CONTINUING CALIBRATION DATA

Lab Name: TESTAMERICA BURLINGTON

Contract: $8 \mathrm{E}-00302$

Lab Code: STLV Case No.: CNTRALIA Mod. Ref No.:

SDG No.: 136697

Instrument ID: J.i

Calibration Date: 04/10/2010 Time: 1925

Lab File ID: JAQ05GC1

Init. Calib. Date(s): 04/05/2010 04/05/2010

EPA Sample No. (VSTD\#\#\#\#): VSTD0050J

Init. Calib. Time(s): $1318 \quad 1505$

Heated Purge: (Y/N)N GC Column: DB-624 ID: 0.53 (mm) Length: 75

(m)

Purge Volume: 25.0

(mL)

\begin{tabular}{|c|c|c|c|c|c|}
\hline COMPOUND & $\overline{\mathrm{RRF}}$ & RRF5. 0 & $\begin{array}{l}\text { MIN } \\
\text { RRF }\end{array}$ & $\because D$ & MAX $\% D$ \\
\hline $\begin{array}{l}================ \\
\text { Vinyl chloride-d3 }\end{array}$ & 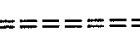 & $======$ & $===== \pm$ & & $=======$ \\
\hline Vinyl chloride-d3 & 0.411 & 0.424 & 0.010 & 3.2 & 50.0 \\
\hline Chloroethane-d5 & 0.310 & 0.324 & 0.010 & 4.4 & 50.0 \\
\hline 1,1-Dichloroethene-d2 & 0.683 & 0.696 & 0.010 & 1.9 & 50.0 \\
\hline 2-Butanone-d5 & 0.027 & 0.025 & 0.010 & -8.5 & 50.0 \\
\hline Chloroform-d & 0.541 & 0.543 & 0.010 & 0.3 & 50.0 \\
\hline 1,2-Dichloroethane-d4 & 0.169 & 0.163 & 0.010 & -3.7 & 50.0 \\
\hline Benzene-d6 & 1.760 & 1.790 & 0.010 & 1.7 & 50.0 \\
\hline 1,2-Dichloropropane-d6 & 0.499 & 0.498 & 0.010 & -0.1 & 50.0 \\
\hline Toluene-d8 & 1.583 & 1.628 & 0.010 & 2.8 & 50.0 \\
\hline trans-1,3-Dichloropropene-d4 & 0.312 & 0.300 & 0.010 & -3.8 & 50.0 \\
\hline $2-$ Hexanone-d5 & 0.033 & 0.030 & 0.010 & $-10 \cdot 5$ & 50.0 \\
\hline $1,1,2,2$-Tetrachloroethane-d2 & 0.151 & 0.144 & 0.010 & -4.7 & 50.0 \\
\hline 1,2-Dichlorobenzene-d4 & 0.764 & 0.759 & 0.010 & -0.6 & 50.0 \\
\hline
\end{tabular}

Report 1,4-Dioxane-d8 for Low-Medium VOA analysis only 
$8 A$ - FORM VIII VOA

VOLATILE INTERNAL STANDARD AREA AND RETENTION TIME SUMMARY

Lab Name: TESTAMERICA BURLINGTON

Contract: $8 \mathrm{E}-00302$

Lab Code: STLV Case No.: CNTRALIA Mod. Ref No.:

SDG No.: 136697

GC Column: DB-624 ID: 0.53 (mm) Init. Calib. Date(s) : 04/05/2010 04/05/2010

EPA Sample No. (VSTD\#\#\#\#) : VSTD005JM Date Analyzed: 04/08/2010

Lab File ID (Standard): JAQ005EV

Time Analyzed: 0852

Instrument ID: J.i

Heated Purge: $(\mathrm{Y} / \mathrm{N}) \mathrm{N}$

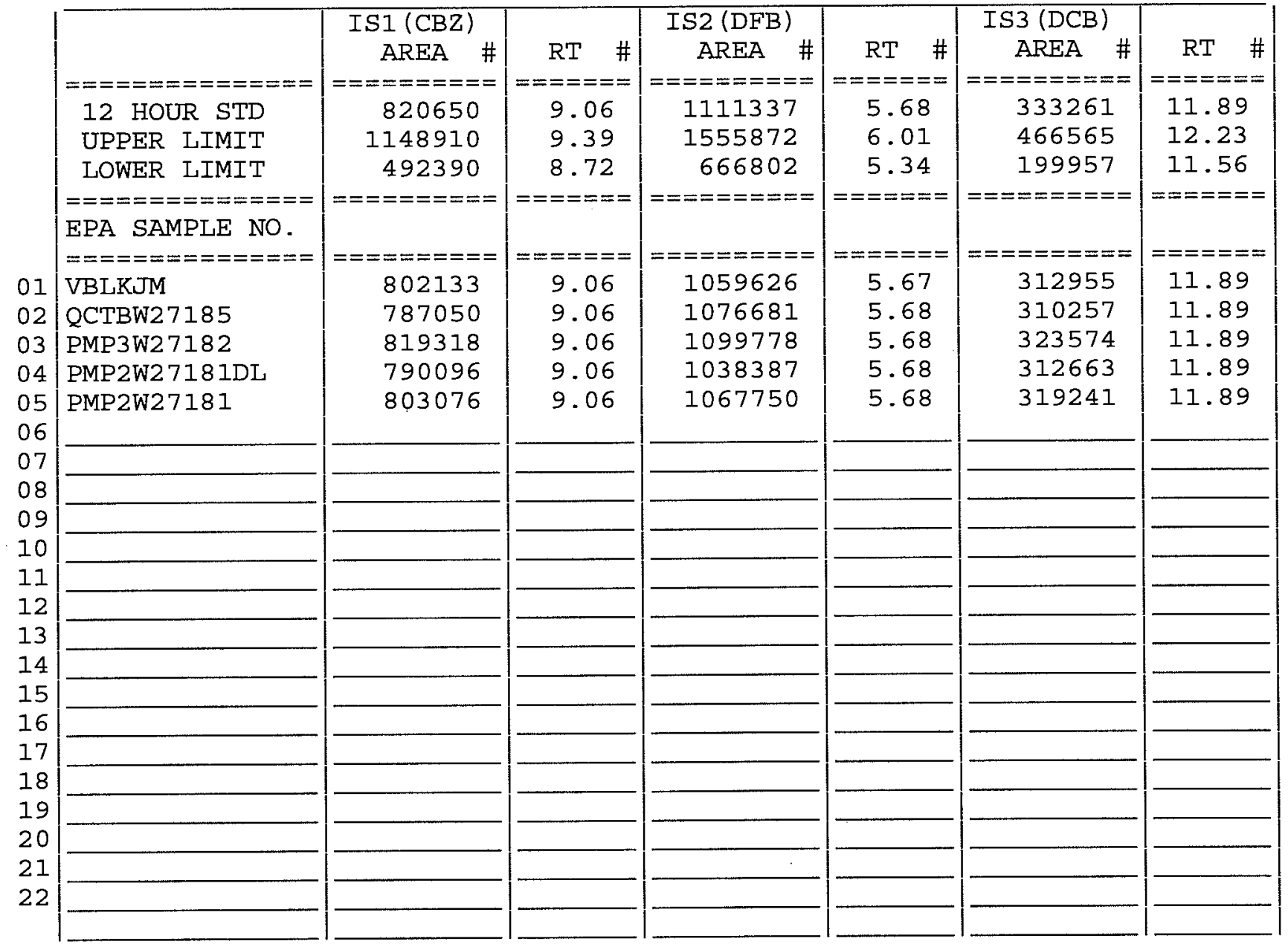

IS1 (CBZ) = Chlorobenzene $-\mathrm{d} 5$

IS2 $(\mathrm{DFB})=1,4$-Difluorobenzene

IS3 $(\mathrm{DCB})=1,4$-Dichlorobenzene-d4

AREA UPPER LIMIT $=200 \%$ (Low-Medium Volatiles) and $140 \%$ (Trace Volatiles) of internal standard area

AREA LOWER LIMIT $=50 \%$ (Low-Medium Volatiles) and $60 \%$ (Trace Volatiles) of internal standard area

RT UPPER LIMIT $=+0.50$ (Low-Medium Volatiles) and +0.33 (Trace Volatiles) minutes of internal standard RT

RT LOWER LIMIT $=-0.50$ (Low-Medium Volatiles) and -0.33 (Trace Volatiles) minutes of internal standard RT

\# Column used to flag values outside QC limits with an asterisk

Page 1 of 1 
$8 A$ - FORM VIII VOA

VOLATILE INTERNAL STANDARD AREA AND RETENTION TIME SUMMARY

Lab Name: TESTAMERICA BURLINGTON

Lab Code: STLV Case No.: CNTRALIA Mod. Ref No.:
SDG NO.: 136697

Init. Calib. Date(s): 04/05/2010 04/05/2010
EPA Sample No. (VSTD\#\#\#\#\#) : VSTD005JN

Lab File ID (Standard): JAQ002FV

Instrument ID: J.i
Contract: $8 \mathrm{E}-00302$

Time Analyzed: 0806

Heated Purge: (Y/N) N

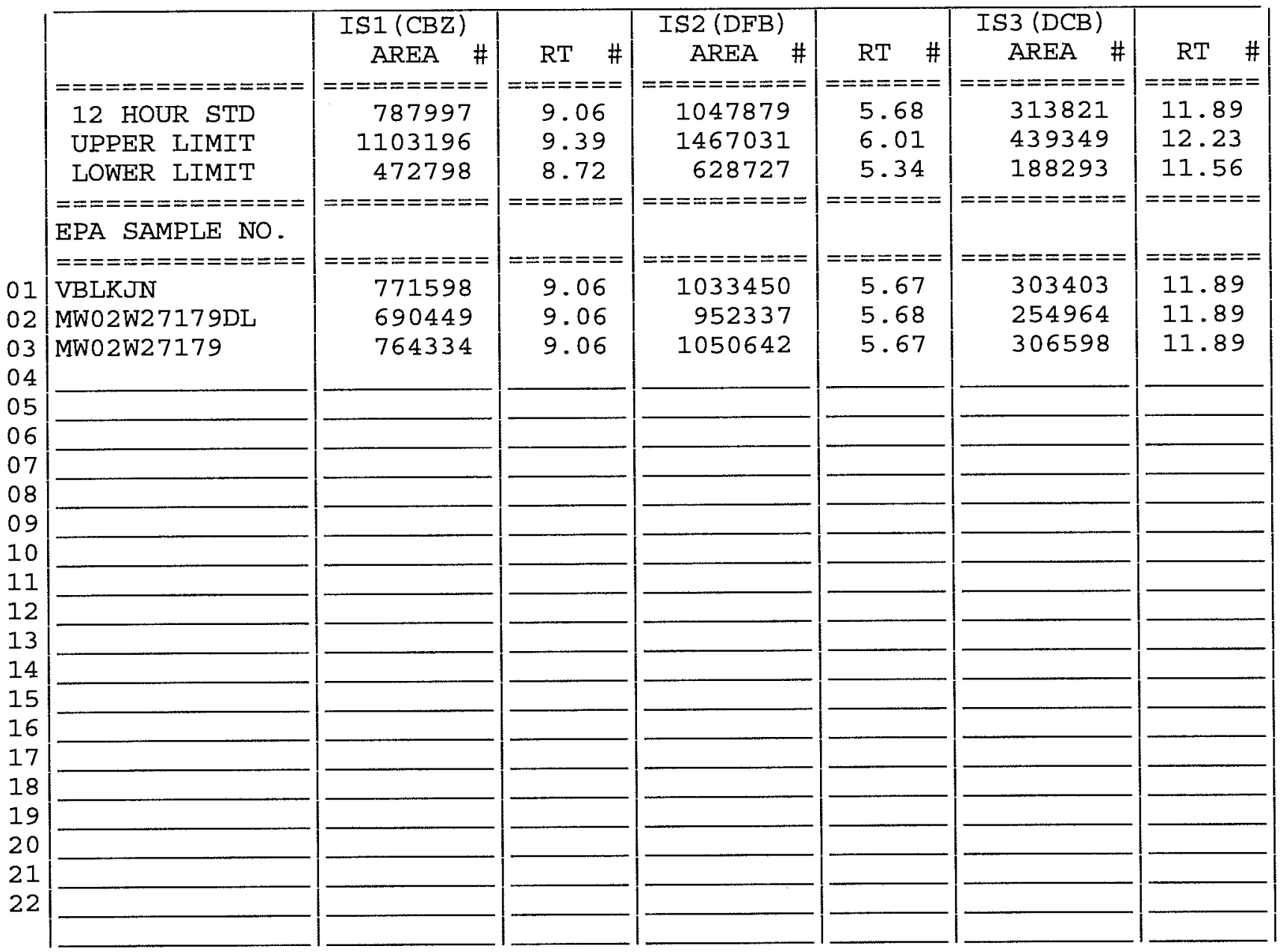

IS1 $(\mathrm{CBZ})=$ Chlorobenzene $-\mathrm{d} 5$

IS2 $(\mathrm{DFB})=1,4$-Difluorobenzene

IS3 $(\mathrm{DCB})=1,4$-Dichlorobenzene-d4

AREA UPPER LIMIT $=200 \%$ (Low-Medium Volatiles) and $140 \%$ (Trace Volatiles) of internal standard area

AREA LOWER LIMIT $=50 \%$ (LOW-Medium Volatiles) and 60\% (Trace Volatiles) of internal standard area

RT UPPER LIMIT $=+0.50$ (Low-Medium Volatiles) and +0.33 (Trace Volatiles) minutes of internal standard RT

RT LOWER LIMIT $=-0.50$ (Low-Medium Volatiles) and -0.33 (Trace Volatiles) minutes of internal standard RT

\# Column used to flag values outside QC limits with an asterisk

Page 1 of 1 
$8 A$ - FORM VIII VOA

VOLATILE INTERNAL STANDARD AREA AND RETENTION TIME SUMMARY

Lab Name: TESTAMERICA BURLINGTON

Contract : $8 E-00302$

Lab Code: STLV Case No.: CNTRALIA Mod. Ref No.:

SDG No.: 136697

GC Column: DB-624 ID: 0.53 (mm) Init. Calib. Date (s): 04/05/2010 04/05/2010

EPA Sample No. (VSTD\#\#\#\#\#) : VSTD005JO

Date Analyzed: 04/10/2010

Lab File ID (Standard) : JAQ005GV

Time Analyzed: 1015

Instrument ID: J.i

Heated Purge: ( $\mathrm{Y} / \mathrm{N}) \mathrm{N}$

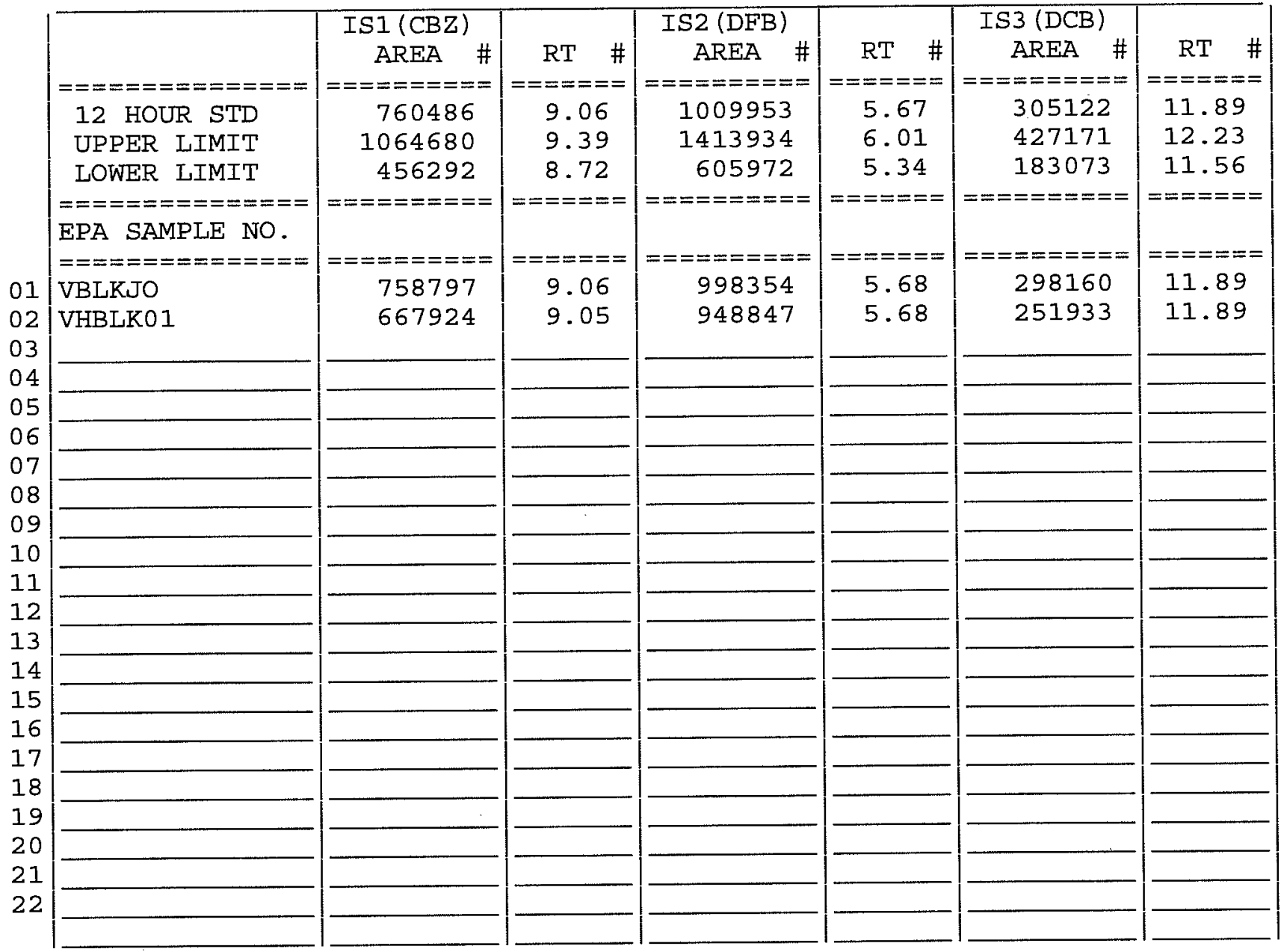

IS1 (CBZ) = Chlorobenzene-d5

IS2 (DFB) = 1,4-Difluorobenzene

IS3 $(D C B)=1,4-$ Dichlorobenzene-d4

AREA UPPER LIMIT $=200 \%$ (Low-Medium Volatiles) and $140 \%$ (Trace Volatiles) of internal standard area

AREA LOWER LIMIT $=50 \%$ (Low-Medium Volatiles) and $60 \%$ (Trace Volatiles) of internal standard area

RT UPPER LIMIT $=+0.50$ (Low-Medium Volatiles) and +0.33 (Trace Volatiles) minutes of internal standard RT

RT LOWER LIMIT $=-0.50$ (Low-Medium Volatiles) and -0.33 (Trace Volatiles) minutes of internal standard RT

\# Column used to flag values outside QC limits with an asterisk Page 1 of 1 


\title{
TestAmerica
}

THE LEADER IN ENYIRONMENTAL TESTING

TestAmerica Laboratories, Inc.

\author{
CASE NARRATIVE \\ Client: Argonne National Laboratory \\ Project: CENTRALIA (200-1629)
}

Report Number: 200-1629-1

With the exceptions noted as flags or footnotes, standard analytical protocols were followed in the analysis of the samples and no problems were encountered or anomalies observed.

Calculations are performed before rounding to avoid round-off errors in calculated results.

All holding times were met and proper preservation noted for the methods performed on these samples, unless otherwise detailed in the individual sections below.

\section{Receipt}

The samples were received on 9/21/2010. Documentation of the condition of the samples at the time of their receipt and any exception to the laboratory's Sample Acceptance Policy is documented in the Sample Handling section of this submittal. The samples, as received, were not acid preserved. On that basis, the laboratory did provide for the analytical work to be performed within seven days of sample collection.

\section{SOM01.2 Volatile Organics (Trace Level Water)}

A storage blank was prepared for volatile organics analysis, and stored in association with the storage of the samples. That storage blank, identified as VHBLK01, was carried through the holding period with the samples, and analyzed.

Each sample was analyzed without a dilution. Each of the analyses associated with the sample set exhibited an acceptable internal standard performance. There was an acceptable recovery of each deuterated monitoring compound (DMC) in the analysis of the method blanks associated with the analytical work, and in the analysis of the storage blank associated with the sample set. The analysis of the samples in this sample set did meet the technical acceptance criteria specific to DMC recoveries, although not all DMC recoveries were within the control range in each analysis. The technical acceptance criteria does provide for the recovery of up to three DMCs to fall outside of the control range in the analysis of field samples. Matrix spike and matrix spike duplicate analyses were not performed on samples in this sample set. Trace concentrations of acetone and 1,2,3-trichlorobenzene were identified in the analysis of each method blank associated with the analytical work. The concentration of each compound in each analysis was below the established reporting limit, and each analysis did meet the technical acceptance criteria for a compliant method blank analysis. A trace concentration of 


\section{TestAmerica}

THE LEADER IN ENVIRONMENTAL TESTING

acetone was identified in the analysis of the storage blank associated with the sample set. The concentration of acetone in that analysis was below the established reporting limit, and the analysis did meet the technical acceptance criteria for a compliant storage blank analysis. Present in the method blank and storage blank analyses was a non-target constituent that represents a compound that is related to the DMC formulation. The fact that the presence of this compound is not within the laboratory's control is at issue. The derived results for that compound have been qualified with an " $\mathrm{X}$ " qualifier to reflect the source of the contamination.

The responses for each of the target analytes met the relative standard deviation criterion in the initial calibration. The response for each target analyte met the percent difference criterion in each continuing calibration check acquisition. The response for each target analyte met the 50.0 percent difference criterion in each closing calibration check acquisition.

The primary quantitation mass for methylcyclohexane that is specified in the Statement of Work is mass 83 . The laboratory did identify a contribution to mass 83 from 1,2-dichloropropane- $d_{6}$, one of the deuterated monitoring compounds (DMCs). The laboratory did change the primary quantitation mass assignment to mass 55 for the quantification of methylcyclohexane.

Manual integration was employed in deriving certain of the analytical results. The values that have been derived from manual integration are qualified on the quantitation reports. Extracted ion current profiles for each manual integration are included in the data package, and further documented in the Sample Preparation section of this submittal.

The analytical results associated with the samples presented in this test report were generated under a quality system that adheres to requirements specified in the NELAC standard. Release of the data in this test report and any associated electronic deliverables is authorized by the Laboratory Director's designee as verified by the following signature.

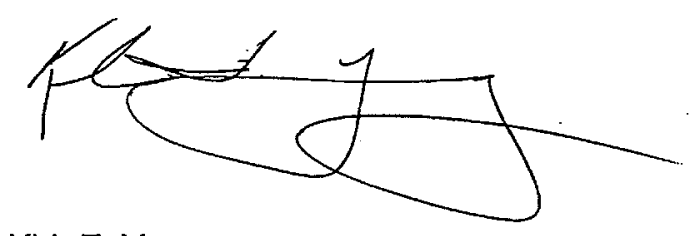

Kirk F. Young

Project Manager 


\section{8}

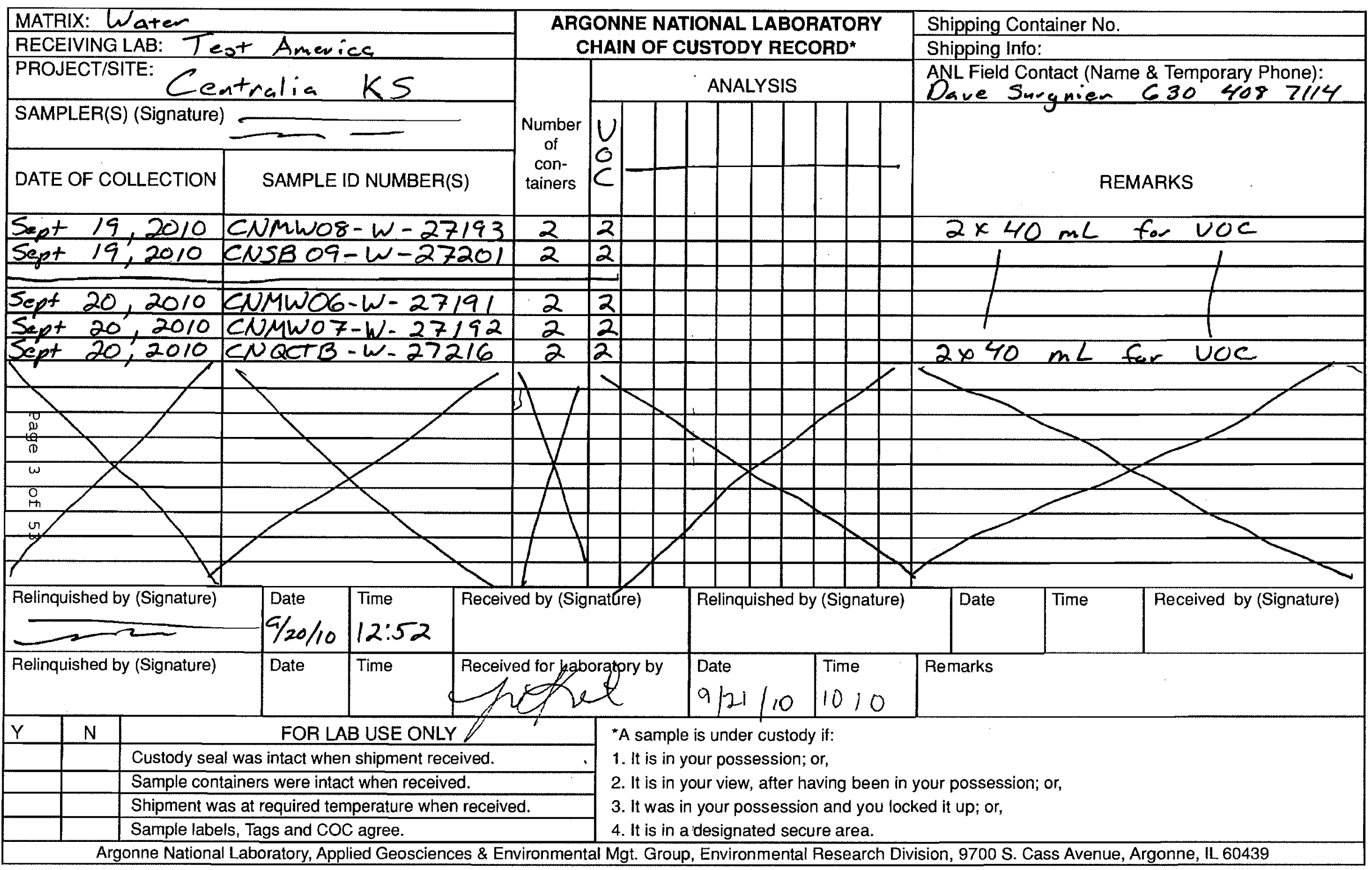


Burlington Facility

Internal Chain of Custody Log (ICOC)

Project Information:

LOG-IN NUMBER: $200-1629$

CLIENT: Argonne

Method: $50 \mathrm{MO1.2}$ - Vol_- Trace

LAB IDs: $200-1629-1$ thru $200-1629-5$

Samples associated with this Log-in were placed into storage on

$\frac{09 / 21 / 10}{\left.\frac{1600}{\left(T_{1}\right)}{ }^{2}\right)}$ by:

Storage Location:

VoA Refrigerator $B$, shelf

Sample Custodian Signature

Specify storage location (refrigerator, freezer 10 or lab location) for oniginal sample containers Internal Transfer:Information

\begin{tabular}{|c|c|c|c|c|c|c|c|c|c|c|}
\hline \multicolumn{2}{|c|}{ Sample Type } & \multirow[t]{2}{*}{ Lab ID(s) } & \multirow{2}{*}{$\begin{array}{c}\text { Transfer } \\
\text { Date }\end{array}$} & \multirow{2}{*}{$\begin{array}{c}\text { Transfer } \\
\text { Time }^{2}\end{array}$} & \multicolumn{3}{|c|}{ Purpose of Transfer } & \multirow{2}{*}{$\begin{array}{l}\text { Relinquished } \\
\text { By: }\end{array}$} & \multirow{2}{*}{$\begin{array}{c}\text { Received } \\
\text { By: }\end{array}$} & \multirow{2}{*}{$\begin{array}{l}\text { Storage Location } \\
\text { Prepared Sample }^{1}\end{array}$} \\
\hline Original & Prepared & & & & Prep & Analysis & Storage & & & \\
\hline$\sim$ & & 1.5 & $9 \cdot 21 \cdot 10$ & 1635 & $r$ & & & Romar logksar & Domer betses & vaA Prep \\
\hline$\sim$ & & $1-5$ & $9-21 \cdot 10$ & 1653 & & & $\alpha$ & Ihomar lecksis & Themes fection & VoA Fridge \\
\hline$\frac{d}{4}$ & & $i$ & $7 / 23110$ & 1015 & & $\sim$ & & JW & Sh 5 & Analysis \\
\hline $\begin{array}{l}0 \\
0 \\
0 \\
0\end{array}$ & & 1 & $9|22|_{10}$ & 1630 & & & L & Ja 5 & $\sqrt{4} 5$ & storese \\
\hline \multicolumn{11}{|l|}{$\mapsto$} \\
\hline \multicolumn{11}{|l|}{$\begin{array}{l}O \\
\text { in } \\
\end{array}$} \\
\hline \multicolumn{11}{|l|}{$\bar{w}$} \\
\hline & & & & & & & & & & \\
\hline & & & & & & & & & & \\
\hline & . & & & & & & & & & \\
\hline & & & & & & & & & & 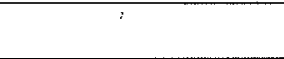 \\
\hline & & & & & & & & & & $\cdot$ \\
\hline & & & & & & & & & & \\
\hline & & & & & & & & & & \\
\hline & & & & & & & & & & \\
\hline & & & & & & & & & & \\
\hline & & & & & & & & & & \\
\hline
\end{tabular}

'Extract, cligestate, or any other prepared sample that is no longer in original sample container

${ }^{2}$ Military Time 


\section{Burlington Facility}

Internal Chain of Custody Log (ICOC)

Project Information:

LOG-IN NUMBER: $200-1629$

CLIENT: Argonne

Method: $H B-50 M 01.2$ - Vol-Trace

LAB IDS: $200-1629-6$

Samples associated with this Log-in were placed into storage on $\frac{09 / 21 / 10}{(0 a t e)} \frac{1600}{\left(\text { Time }^{2}\right)}$ by:

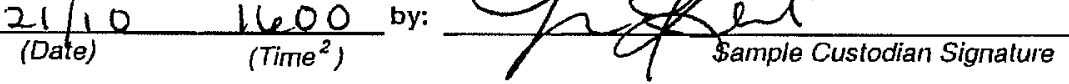

Storage Location: VOA Refrige rator $B$, Shel $F 8$ specify slorage location (refrigerator, freezer 10 or lab location) for original sample containers Storage Condition: /Refrigeration Frozen Ambient

Internal Transfer:Information

\begin{tabular}{|c|c|c|c|c|c|c|c|c|c|c|}
\hline \multicolumn{2}{|c|}{ Sample Type } & \multirow[t]{2}{*}{ Lab ID(s) } & \multirow{2}{*}{$\begin{array}{c}\text { Transfer } \\
\text { Date }\end{array}$} & \multirow{2}{*}{$\begin{array}{c}\text { Transfer } \\
\text { Time }^{2} \\
\end{array}$} & \multicolumn{3}{|c|}{ Purpose of Transfer } & \multirow{2}{*}{$\begin{array}{c}\text { Relinquished } \\
\text { By: }\end{array}$} & \multirow{2}{*}{$\begin{array}{l}\text { Received } \\
\text { By: }\end{array}$} & \multirow{2}{*}{$\begin{array}{l}\text { Storage Location } \\
\text { Prepared Sample }\end{array}$} \\
\hline Original & Prepared ${ }^{1}$ & & & & Prep & Analysis & Storage & & & \\
\hline$\checkmark$ & & 6 & $9-21-10$ & 1635 & $\sim$ & & & Deomas Reto & Themertates & VoA Prep \\
\hline 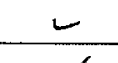 & & 6 & $9 \cdot 21-10$ & 1653 & & & $\sim$ & Theourkeksu & cs lectes & vaA fridge \\
\hline$\frac{\sigma}{0}$ & & 11 & $9|24| c 0$ & 0710 & & 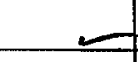 & & $=7$ & TI & Analysis \\
\hline$\stackrel{D}{0}_{0}^{0}$ & & $M$ & $9|2 y| 10$ & 0715 & & & $\longleftarrow$ & 74 & 5 & Stonche \\
\hline G & & & & & & & & & & \\
\hline $\begin{array}{l}O \\
P\end{array}$ & & & & & & & & & & \\
\hline 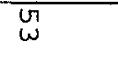 & & & & & & & & & & \\
\hline & & & & & & & & & & \\
\hline & & & & & & & & & & \\
\hline & & & & & & & & & & \\
\hline & & & & & & & & & & \\
\hline & & & & & & & & & & \\
\hline & & & & & & & & & & \\
\hline & & & & & & & & & & \\
\hline & & & & & & & & & & \\
\hline & & & & & & & & & & \\
\hline & & & & & & & & & & \\
\hline
\end{tabular}

'Extract, digestate, or any other prepared sample that is no longer in original sample contalner

2 Military Time 
$2 A-F O R M I I V O A-1$

WATER VOLATILE DEUTERATED MONITORING COMPOUND RECOVERY

Lab Name: TESTAMERICA BURLINGTON

Contract: $8 \mathrm{E}-00302$

Lab Code: STLV_Case No.: CENTRA Mod. Ref No.:

SDG NO.: 200-1629

Level: (TRACE or LOW) TRACE

\begin{tabular}{|c|c|c|c|c|c|c|c|}
\hline $\begin{array}{c}\text { EPA } \\
\text { SAMPLE NO. }\end{array}$ & $\begin{array}{l}\text { VDMCI } \\
\text { (VCL) \# }\end{array}$ & $\begin{array}{l}\text { VDMC2 } \\
\text { (CLA) \# }\end{array}$ & $\begin{array}{l}\text { VDMC3 } \\
\text { (DCE) \# }\end{array}$ & $\begin{array}{l}\text { VDMC4 } \\
\text { (BUT) \# }\end{array}$ & $\begin{array}{l}\text { VDMC5 } \\
\text { (CLF) \# }\end{array}$ & $\begin{array}{l}\text { VDMC6 } \\
\text { (DCA) } \#\end{array}$ & $\begin{array}{c}\text { VDMC7 } \\
(\mathrm{BEN}) \quad \#\end{array}$ \\
\hline VBLKJE & 101 & 101 & 77 & 103 & 102 & 108 & 105 \\
\hline CNMW08-S-27193 & 102 & 103 & 80 & 132 & 105 & 107 & 108 \\
\hline CNSB09-W-27201 & 98 & 100 & 78 & 123 & 103 & 110 & 104 \\
\hline CNMW06-W-27191 & 100 & 101 & 77 & 121 & 102 & 109 & 106 \\
\hline CNMW07-W-27192 & 102 & 102 & 79 & 152 & 105 & 108 & 109 \\
\hline CNQCTB-W-27216 & 98 & 100 & 78 & $212 *$ & 100 & 106 & 106 \\
\hline VBLKJG & 97 & 98 & 76 & 98 & 100 & 103 & 104 \\
\hline VHBLKO1 & 104 & 105 & 81 & 104 & 103 & 107 & 109 \\
\hline
\end{tabular}

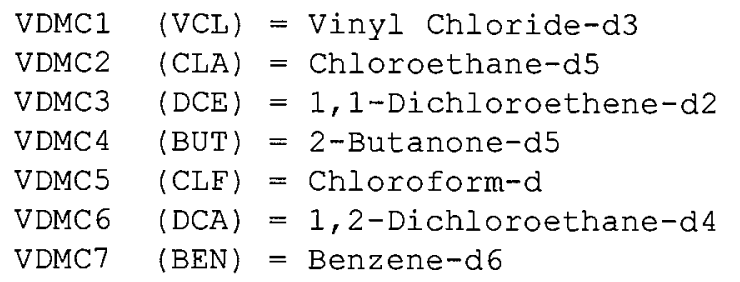

\# Column to be used to flag recovery values

* Values outside of contract required QC limits
QC LIMITS

$$
\begin{aligned}
& (65-131) \\
& (71-131) \\
& (55-104) \\
& (49-155) \\
& (78-121) \\
& (78-129) \\
& (77-124)
\end{aligned}
$$

Page 1 of 1 
$2 B$ - FORM II VOA-2

WATER VOLATILE DEUTERATED MONITORING COMPOUND RECOVERY

Lab Name: TESTAMERICA BURLINGTON

Contract: $8 \mathrm{E}-00302$

Lab Code: STLV Case No.: CENTRA Mod. Ref No.:

SDG No.: 200-1629

Level: (TRACE or LOW) TRACE

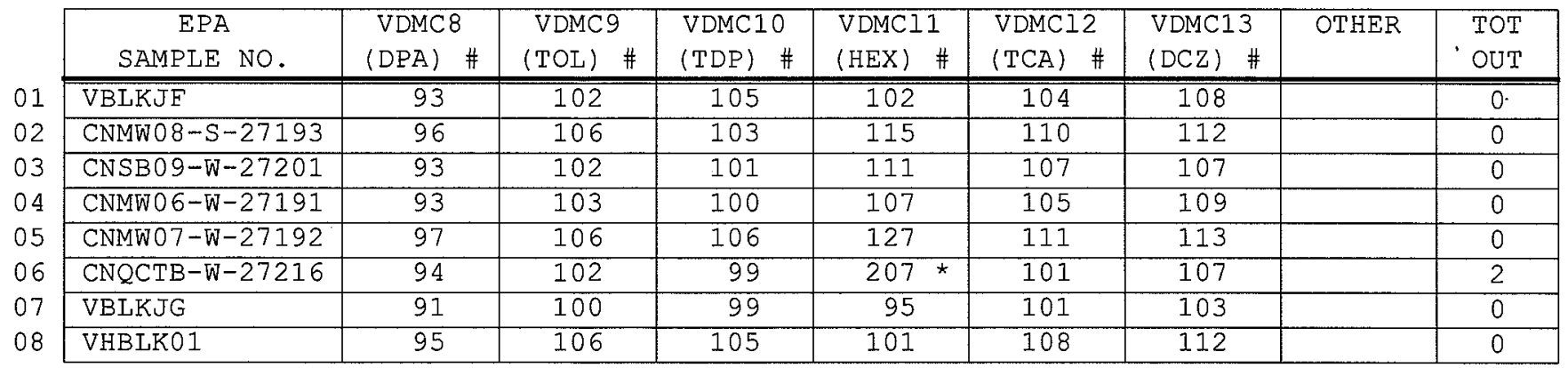

VDMC8 $\quad(D P A)=1,2-$ Dichloropropane-d 6

QC LIMITS

VDMC 9 (TOL) = Toluene-d8

$(79-124)$

VDMC10 $(T D P)=$ trans $-1,3-D i c h l o r o p r o p e n e-d 4$

$(77-121)$

$(73-121)$

VDMC11 $(\mathrm{HEX})=2-$ Hexanone-d5

VDMC12 $(\mathrm{TCA})=1,1,2,2-\mathrm{Tet}$ rachloroethane-d2

$(28-135)$

$(73-125)$

VDMC13 $(\mathrm{DCZ})=1,2-\mathrm{Dichlorobenzene-d4}$

$(80-131)$

\# Column to be used to flag recovery values

* Values outside of contract required QC limits

Report 1,4-Dioxane-d8 for Low-Medium VOA analysis only

Page 1 of 1

SOM01.2 (4/2007) 
$4 A$ - FORM IV VOA

VOLATILE METHOD BLANK SUMMARY
EPA SAMPLE NO.

VBLKJF

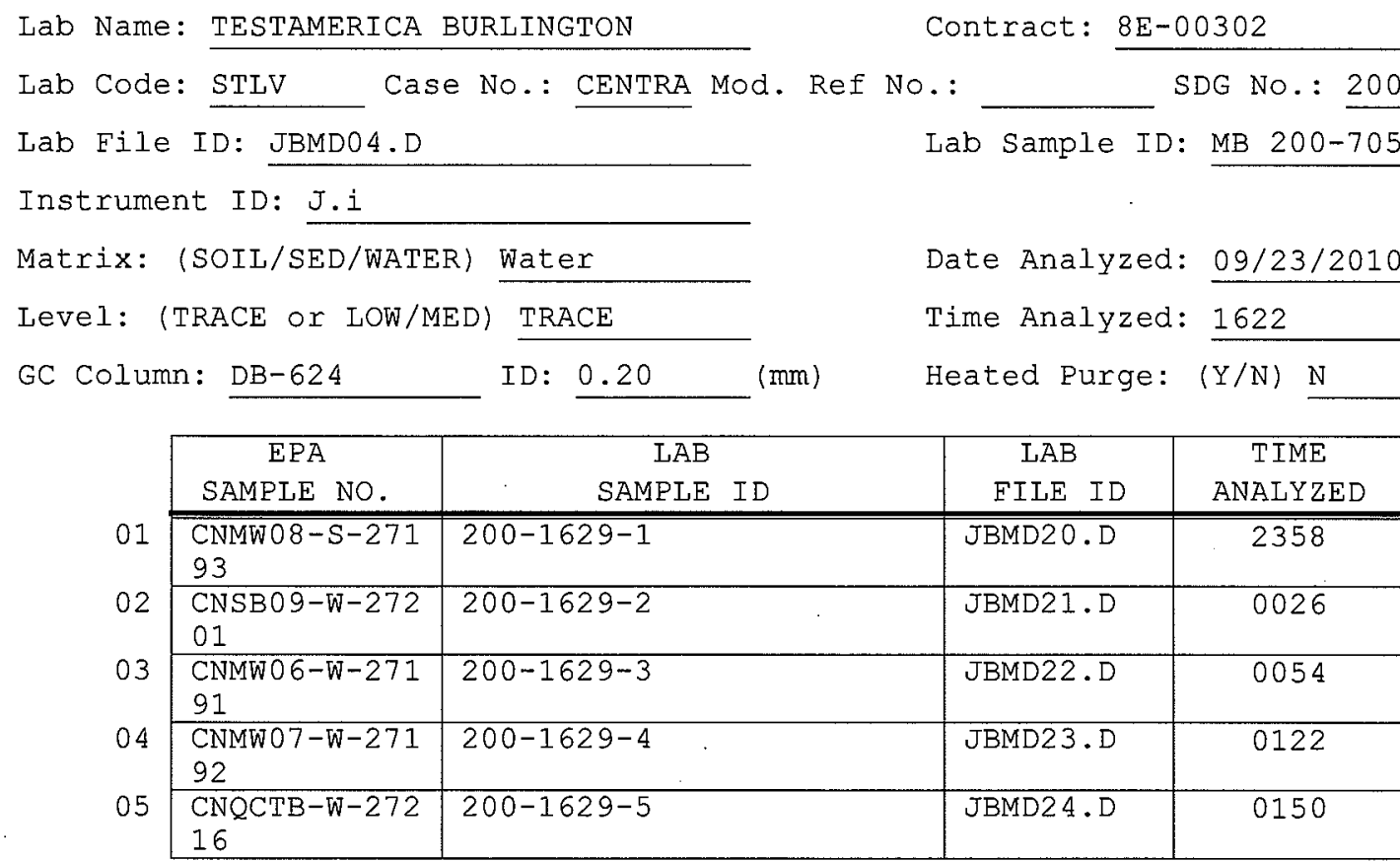

COMMENTS:

Page 1 of 1 
$\triangle A$ - FORM IV VOA

VOLATILE METHOD BLANK SUMMARY
EPA SAMPLE NO.

VBLKJG
Làb Name: TESTAMERICA BURLINGTON

Lab Code: STLV

Lab File ID: JBME04.D Case No.: CENTRA Mod. Ref No.:

Contract: $8 \mathrm{E}-00302$

Instrument ID: J.i

Matrix: (SOIL/SED/WATER) Water

Level: (TRACE or LOW/MED) TRACE

GC Column: DB-624

ID : 0.20

(nm)
SDG No.: 200-1629

Lab Sample ID: MB 200-7048/4

Date Analyzed: 09/24/2010

Time Analyzed: 0737

Heated Purge: ( $Y / N$ ) N
01

\begin{tabular}{|c|c|}
\hline EPA & LAB \\
SAMPLE NO. & SAMPLE ID \\
\hline VHBLKO1 & $200-1629-6$
\end{tabular}

TIME ANALYZED

0842

COMMENTS :

Page 1 of 1 
$5 A$ - FORM V VOA

VOLATILE ORGANICS INSTRUMENT

PERFORMANCE CHECK

BROMOFLUOROBENZENE (BFB)
EPA SAMPLE NO.

BFBJB
Lab Name: TESTAMERICA BURLINGTON

Lab Code: STLV

Lab File Id: JBM01.D

Instrument Id: J.i

GC Column: DB-624

ID: 0.20
Contract: $8 \mathrm{E}-00302$

SDG No.: 200-1629

BFB Injection Date: 09/21/2010

BFB Injection Time: 1417 (mm)

\begin{tabular}{|r|l|r|}
\hline m/e & ION ABUNDANCE CRITERIA & $\begin{array}{c}\text { RELATIVE } \\
\text { ABUNDANCE }\end{array}$ \\
\hline \hline 50 & $15.0-40.0 \%$ of mass 95 & 16.2 \\
\hline 75 & $30.0-80.0 \%$ of mass 95 & 51.0 \\
\hline 95 & Base peak, 100\% relative abundance & 100 \\
\hline 96 & $5.0-9.0 \%$ of mass 95 & 6.6 \\
\hline 173 & Less than 2.0\% of mass 174 & $0.6(1)$ \\
\hline 174 & $50.0-120 \%$ of mass 95 & 68.5 \\
\hline 175 & $5.0-9.0 \%$ of mass 174 & $7.1) 1$ \\
\hline 176 & $95.0-101 \%$ of mass 174 & $88.5(100) 1$ \\
\hline 177 & $5.0-9.0 \%$ of mass 176 & $6.6) 2$ \\
\hline
\end{tabular}

1 - Value is omass $174 \quad 2$ - Value is omass 176

\begin{tabular}{|c|c|c|l|c|c|}
\cline { 2 - 7 } & \multicolumn{1}{|c|}{$\begin{array}{c}\text { EPA } \\
\text { SAMPLE NO. }\end{array}$} & \multicolumn{1}{c|}{$\begin{array}{c}\text { LAB } \\
\text { SAMPIE ID }\end{array}$} & $\begin{array}{c}\text { DATE } \\
\text { FILE ID }\end{array}$ & TIME \\
\cline { 2 - 7 } 01 & VSTD0.5JB & IC 200-6837/2 & JBM02.D & 09/21/2010 & 1435 \\
\cline { 2 - 7 } 02 & VSTD001JB & IC 200-6837/3 & JBM03.D & $09 / 21 / 2010$ & 1503 \\
\cline { 2 - 7 } 03 & VSTD005JB & ICIS 200-6837/4 & JBM04.D & $09 / 21 / 2010$ & 1531 \\
\cline { 2 - 7 } 04 & VSTD010JB & IC 200-6837/5 & JBM05.D & $09 / 21 / 2010$ & 1559 \\
\cline { 2 - 7 } & VSTD020JB & IC 200-6837/8 & JBM08.D & $09 / 21 / 2010$ & 1724 \\
\hline
\end{tabular}

Page 1 of 1 
$5 A-F O R M V V O A$

VOLATILE ORGANICS INSTRUMENT

PEREORMANCE CHECK

BROMOELUOROBENZENE (BEB)
EPA SAMPLE NO.

BEBJF
Lab Name: TESTAMERICA BURLINGTON Case No.: CENTRA Mod. Ref No.:
Contract: $8 \mathrm{E}-00302$

SDG No.: $200-1629$

BEB Injection Date: 09/23/2010

BFB Injection Time: 1514

GC Column: $\mathrm{DB}-624$

ID: 0.20 (mm)

\begin{tabular}{|c|c|c|}
\hline $\mathrm{m} / \mathrm{e}$ & ION ABUNDANCE CRITERIA & $\begin{array}{l}\text { RELATIVE } \\
\text { ABUNDANCE }\end{array}$ \\
\hline 50 & $15.0-40.0 \%$ of mass 95 & 17.0 \\
\hline 75 & $30.0-80.0 \%$ of mass 95 & 49.8 \\
\hline 95 & Base peak, $100 \%$ relative abundance & 100 \\
\hline 96 & $5.0-9.0 \%$ of mass 95 & 6.6 \\
\hline 173 & Less than $2.0 \%$ of mass 174 & $0.5) 1$ \\
\hline 174 & $50.0-120 \%$ of mass 95 & 89.8 \\
\hline 175 & $5.0-9.0 \%$ of mass 174 & $6.2(6.9) 1$ \\
\hline 176 & $95.0-101 \%$ of mass 174 & $89.4(99.5) 1$ \\
\hline 177 & $5.0-9.0 \%$ of mass 176 & $5.8(6.5) 2$ \\
\hline
\end{tabular}

1 - Value is omass $174 \quad 2$ - Value is omass 176

\begin{tabular}{|c|c|c|c|c|}
\hline $\begin{array}{c}\text { EPA } \\
\text { SAMPLE NO. }\end{array}$ & $\begin{array}{c}\text { LAB } \\
\text { SAMPLE ID }\end{array}$ & $\begin{array}{c}\text { LAB } \\
\text { FILE ID }\end{array}$ & $\begin{array}{c}\text { DATE } \\
\text { ANALYZED }\end{array}$ & $\begin{array}{c}\text { TIME } \\
\text { ANALYZED }\end{array}$ \\
\hline VSTD005JE & CCVIS $200-7052 / 2$ & JBMD02.D & $09 / 23 / 2010$ & 1526 \\
\hline VBLKJF & MB $200-7052 / 4$ & JBMD0 4.D & $09 / 23 / 2010$ & 1622 \\
\hline $\begin{array}{l}\text { CNMW08-S-2 } \\
7193\end{array}$ & $200-1629-1$ & JBMD20.D & $09 / 23 / 2010$ & 2358 \\
\hline $\begin{array}{l}\text { CNSBO9-W-2 } \\
7201\end{array}$ & $200-1629-2$ & JBMD21.D & $09 / 24 / 2010$ & 0026 \\
\hline $\begin{array}{l}\text { CNMW0 6-W-2 } \\
7191\end{array}$ & $200-1629-3$ & JBMD22 . D & $09 / 24 / 2010$ & 0054 \\
\hline $\begin{array}{l}\text { CNMW 0 7-W-2 } \\
7192\end{array}$ & $200-1629-4$ & JBMD23.D & $09 / 24 / 2010$ & 0122 \\
\hline $\begin{array}{l}\text { CNQCTB-W-2 } \\
7216 \\
\end{array}$ & $200-1629-5$ & JBMD24.D & $09 / 24 / 2010$ & 0150 \\
\hline VSTD005FJ & CCVC $200-7052 / 25$ & JBMD25.D & $09 / 24 / 2010$ & 0218 \\
\hline
\end{tabular}


$5 A$ - FORM V VOA

VOLATILE ORGANICS INSTRUMENT

PEREORMANCE CHECK BROMOFLUOROBENZENE
EPA SAMPLE NO.

BFBJG
Lab Name: TESTAMERICA BURLINGTON

Lab Code: STLV

Iab File Id: JBME01.D

Instrument Id: J.i

GC Column: DB-624

ID: 0.20
Contract: $8 \mathrm{E}-00302$

SDG No.: 200-1629

BFB Injection Date: 09/24/2010

BFB Injection Time: 0622

\begin{tabular}{|r|l|c|}
\hline $\mathrm{m} / \mathrm{e}$ & ION ABUNDANCE CRITERIA & $\begin{array}{c}\text { \% } \\
\text { RELATIVE } \\
\text { ABUNDANCE }\end{array}$ \\
\hline \hline 50 & $15.0-40.0 \%$ of mass 95 & 18.1 \\
\hline 75 & $30.0-80.0 \%$ of mass 95 & 51.7 \\
\hline 95 & Base peak, 100\% relative abundance & 100 \\
\hline 96 & $5.0-9.0 \%$ of mass 95 & 6.3 \\
\hline 173 & Less than 2.0\% of mass 174 & 0.5( \\
\hline 174 & $50.0-120 \%$ of mass 95 & 85.4 \\
\hline 175 & $5.0-9.0 \%$ of mass 174 1 \\
\hline 176 & $95.0-101 \%$ of mass 174 & 6.6( \\
\hline 177 & $5.0-9.0 \%$ of mass 176 & $84.3(98.8) 1$ \\
\hline
\end{tabular}

1 - Value is omass 1742 - Value is omass 176

\begin{tabular}{|l|l|l|l|c|c|}
\hline \multicolumn{1}{|c|}{$\begin{array}{c}\text { EPA } \\
\text { SAMPIE NO. }\end{array}$} & \multicolumn{1}{|c|}{ LAB } & LAB & DATE & TIME \\
01 & \multicolumn{1}{|c|}{ SAMPE ID } & FILE ID & ANALYZED & ANALYZED \\
\cline { 2 - 7 } 02 & VSTD005JG & CCVIS 200-7048/2 & JBME02.D & $09 / 24 / 2010$ & 0641 \\
\cline { 2 - 7 } 03 & VBLKJG & MB 200-7048/4 & JBME04.D & $09 / 24 / 2010$ & 0737 \\
\cline { 2 - 6 } 04 & VHBLK01 & $200-1629-6$ & JBME06.D & $09 / 24 / 2010$ & 0842 \\
\cline { 2 - 7 } & VSTD005GJ & CCVC 200-7048/17 & JBME17.D & $09 / 24 / 2010$ & 1354 \\
\hline
\end{tabular}

Page 1 of 1 
$8 A$ - FORM VIII VOA

VOLATILE INTERNAL STANDARD AREA AND RETENTION TIME SUMMARY

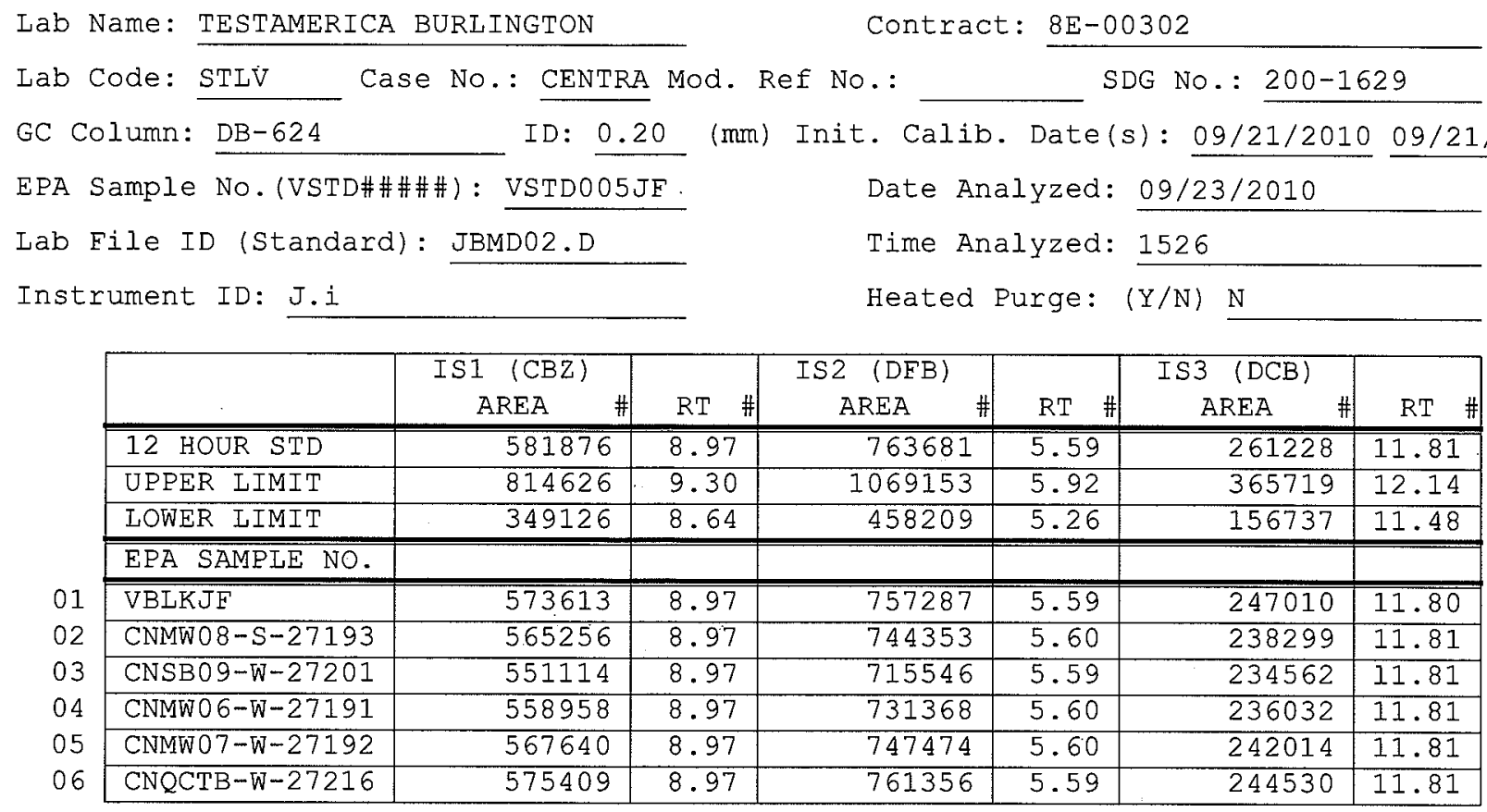

\footnotetext{
IS1 (CBZ) = Chlorobenzene-d5

IS2 $(\mathrm{DFB})=1,4$-Difluorobenzene

IS3 $(\mathrm{DCB})=1,4-\mathrm{Dich}$ lorobenzene-d4
}

AREA UPPER LIMIT $=140 \%$ (Trace Volatiles) of internal standard area AREA LOWER LIMIT $=60 \%$ (Trace Volatiles) of internal standard area RT UPPER LIMIT $=+0.33$ (Trace Volatiles) minutes of internal standard RT RT LOWER LIMIT $=-0.33$ (Trace Volatiles) minutes of internal standard RT

\# Column used to flag values outside contract required QC limits with an asterisk. Page 1 of 1 
$8 \mathrm{~A}-$ FORM VIII VOA

VOLATILE INTERNAL STANDARD AREA AND RETENTION TIME SUMMARY

Lab Name: TESTAMERICA BURLINGTON

Contract: $8 \mathrm{E}-00302$

Lab Code: STLV Case No.: CENTRA Mod. Ref No.: SDG No.: 200-1629

GC Column: $\mathrm{DB}-624$ ID: 0.20 (mm) Init. Calib. Date te (s): $09 / 21 / 2010 \quad 09 / 21 / 2010$

EPA Sample No. (VSTD\#\#\#\#\#): VSTD005JG

Lab File ID (Standard): JBME02.D Date Analyzed: 09/24/2010

Instrument ID: J.i

Time Analyzed: 0641

Heated Purge: ( $\mathrm{Y} / \mathrm{N}) \mathrm{N}$

\begin{tabular}{|c|c|c|c|c|c|c|}
\hline & $\begin{array}{c}\text { IS1 (CBZ) } \\
\text { AREA }\end{array}$ & $\mathrm{RT} \#$ & $\begin{array}{c}\text { IS2 (DFB) } \\
\text { AREA }\end{array}$ & RT \# & $\begin{array}{c}\text { IS3 (DCB) } \\
\text { AREA }\end{array}$ & RT \\
\hline 12 HOUR STD & 588298 & 8.97 & 772826 & 5.59 & 266878 & 11.80 \\
\hline UPPER LIMIT & 823617 & 9.30 & 1081956 & 5.92 & 373629 & 12.13 \\
\hline LOWER LIMIT & 352979 & 8.64 & 463696 & 5.26 & 160127 & 11.47 \\
\hline EPA SAMPLE NO. & & & & & & \\
\hline VBLKJG & 578406 & 8.97 & 761685 & 5.59 & 248197 & 11.81 \\
\hline VHBLKO1 & 543933 & 8.97 & 724734 & 5.59 & 226345 & 11.80 \\
\hline
\end{tabular}

\footnotetext{
IS1 $(\mathrm{CBZ})=$ Chlorobenzene-d5

IS2 $(\mathrm{DFB})=1,4$-Difluorobenzene

IS3 $(\mathrm{DCB})=1,4-\mathrm{Dich}$ lorobenzene-d4
}

AREA UPPER LIMIT $=140 \%$ (Trace Volatiles) of internal standard area AREA LOWER LIMIT $=60 \%$ (Trace Volatiles) of internal standard area RT UPPER LIMIT $=+0.33$ (Trace Volatiles) minutes of internal standard RT RT LOWER LIMIT $=-0.33$ (Trace Volatiles) minutes of internal standard RT

\# Column used to flag values outside contract required QC limits with an asterisk. 
Lab Name: TESTAMERICA BURLINGTON

Iab Code: STLV

Matrix: (SOIL/SED/WATER)

Sample wt/vol: 25.0

Case No.: CENTRA Mod. Ref No.:
Water

$(\mathrm{g} / \mathrm{mL}) \mathrm{mL}$
Contract: $8 \mathrm{E}-00302$

SDG No.: 200-1629
Level: (TRACE/LOW/MED) TRACE

\% Moisture: not dec.

GC Column: DB-624 ID $: 0.20 \quad(\mathrm{~mm})$

Soil Extract Volume:

Lab Sample ID: 200-1629-3

Lab File ID: JBMD22.D

Date Received: 09/21/2010

Date Analyzed: 09/24/2010

Dilution Factor: 1.0

Soil Aliquot Volume:

(uL)
(uL)

(mL)

\begin{tabular}{|c|c|c|c|}
\hline CAS NO. & COMPOUND & $\begin{array}{l}\text { CONCENTRATION UNITS: } \\
(u g / L \text { or } u g / k g) u g / L\end{array}$ & $Q$ \\
\hline $75-71-8$ & Dichlorodifluoromethane & 0.50 & $\bar{U}$ \\
\hline $74-87-3$ & Chloromethane & 0.50 & $\bar{U}$ \\
\hline $75-01-4$ & Vinyl chloride & 0.50 & $\bar{U}$ \\
\hline $74-83-9$ & Bromomethane & 0.50 & $\mathrm{U}$ \\
\hline $75-00-3$ & Chloroethane & 0.50 & $\mathrm{U}$ \\
\hline $75-69-4$ & Trichlorofluoromethane & 0.50 & $\mathrm{U}$ \\
\hline $75-35-4$ & 1,1-Dichloroethene & 0.50 & $\mathrm{U}$ \\
\hline $76-13-1$ & 1, 1,2-Trichloro-1,2,2-trifluoroethane & 0.50 & $\bar{U}$ \\
\hline $67-64-1$ & Acetone & 1.9 & $\mathrm{~J} \mathrm{~B}$ \\
\hline $75-15-0$ & Carbon disulfide & 0.50 & $\mathrm{U}$ \\
\hline $79-20-9$ & Methyl acetate & 0.50 & $\mathrm{U}$ \\
\hline $75-09-2$ & Methylene Chloride & 0.50 & $\mathrm{U}$ \\
\hline $156-60-5$ & trans-1,2-Dichloroethene & 0.50 & $\mathrm{U}$ \\
\hline $1634-04-4$ & Methyl tert-butyl ether & 0.50 & $\mathrm{U}$ \\
\hline $75-34-3$ & 1,1-Dichloroethane & 0.50 & $\mathrm{U}$ \\
\hline $156-59-2$ & cis-1,2-Dichloroethene & 0.50 & $\bar{U}$ \\
\hline $78-93-3$ & 2-Butanone & 5.0 & $\bar{U}$ \\
\hline $74-97-5$ & Bromochloromethane & 0.50 & $\mathrm{U}$ \\
\hline $67-66-3$ & Chloroform & 0.50 & $\mathrm{U}$ \\
\hline $71-55-6$ & 1,1,1-Trichloroethane & 0.50 & $\bar{U}$ \\
\hline $110-82-7$ & Cyclohexane & 0.50 & $\bar{U}$ \\
\hline $56-23-5$ & Carbon tetrachloride & 0.15 & $\mathrm{~J}$ \\
\hline $71-43-2$ & Benzene & 0.50 & $\mathrm{U}$ \\
\hline $107-06-2$ & 1,2-Dichloroethane & 0.50 & $\bar{U}$ \\
\hline
\end{tabular}

Report 1,4-Dioxane for Low-Medium VOA analysis only 
Lab Name: TESTAMERICA BURLINGTON

Contract: 8E-00302

Lab Code: STLV Case No.: CENTRA Mod. Ref No.: SDG NO.: 200-1629

Matrix: (SOIL/SED/WATER) Water

Sample wt/vol: 25.0 $(\mathrm{g} / \mathrm{mL}) \mathrm{mL}$

Level: (TRACE/LOW/MED) TRACE

\% Moisture: not dec.

GC Column: DB-624

ID: 0.20 (mm)

Soil Extract Volume: (UI)

Purge Volume: 25.0 (mL)
Lab Sample ID: 200-1629-3

Lab File ID: JBMD22.D

Date Received: 09/21/2010

Date Analyzed: 09/24/2010

Dilution Factor: 1.0

Soil Aliquot volume: (uL)

\begin{tabular}{|c|c|c|c|}
\hline CAS NO. & COMPOUND & $\begin{array}{l}\text { CONCENTRATION UNITS: } \\
(\mathrm{ug} / \mathrm{L} \text { or } \mathrm{ug} / \mathrm{kg}) \mathrm{ug} / \mathrm{L}\end{array}$ & $Q$ \\
\hline $79-01-6$ & Trichloroethene & 0.50 & $\overline{\mathrm{U}}$ \\
\hline $108-87-2$ & Methylcyclohexane & 0.50 & $\mathrm{U}$ \\
\hline $78-87-5$ & 1,2-Dichloropropane & 0.50 & $\bar{U}$ \\
\hline $75-27-4$ & Bromodichloromethane & 0.50 & $\bar{U}$ \\
\hline $10061-01-5$ & cis-1,3-Dichloropropene & 0.50 & $\mathrm{U}$ \\
\hline $108-10-1$ & 4-Methyl-2-pentanone & 5.0 & $\overline{\mathrm{U}}$ \\
\hline $108-88-3$ & Toluene & 0.035 & $\mathrm{~J}$ \\
\hline $10061-02-6$ & trans-1,3-Dichloropropene & 0.50 & $\mathrm{U}$ \\
\hline $79-00-5$ & 1,1,2-Trichloroethane & 0.50 & $\mathrm{U}$ \\
\hline $127-18-4$ & Tetrachloroethene & 0.50 & $\bar{U}$ \\
\hline $591-78-6$ & 2-Hexanone & 5.0 & $\bar{U}$ \\
\hline $124-48-1$ & Dibromochloromethane & 0.50 & $\overline{\mathrm{U}}$ \\
\hline $106-93-4$ & 1,2-Dibromoethane & 0.50 & $\overline{\mathrm{U}}$ \\
\hline $108-90-7$ & Chlorobenzene & 0.50 & $\mathrm{U}$ \\
\hline $100-41-4$ & Ethylbenzene & 0.50 & $\bar{U}$ \\
\hline $95-47-6$ & o-xylene & 0.50 & $\overline{\mathrm{U}}$ \\
\hline $179601-23-1$ & $m, p-x y l e n e$ & 0.50 & $\overline{\mathrm{U}}$ \\
\hline $100-42-5$ & Styrene & 0.50 & $\mathrm{U}$ \\
\hline $75-25-2$ & Bromoform & 0.50 & $\mathrm{U}$ \\
\hline $98-82-8$ & Isopropylbenzene & 0.50 & $\mathrm{U}$ \\
\hline $79-34-5$ & $1,1,2,2$-Tetrachloroethane & 0.50 & $\mathrm{U}$ \\
\hline $541-73-1$ & 1,3-Dichlorobenzene & 0.50 & $\mathrm{U}$ \\
\hline $106-46-7$ & 1,4-Dichlorobenzene & 0.50 & $\overline{\mathrm{U}}$ \\
\hline $95-50-1$ & 1,2-Dichlorobenzene & 0.50 & $\mathrm{U}$ \\
\hline $96-12-8$ & 1,2-Dibromo-3-Chloropropane & 0.50 & $\mathrm{U}$ \\
\hline $120-82-1$ & $1,2,4$-Trichlorobenzene & 0.50 & $\bar{U}$ \\
\hline $87-61-6$ & $1,2,3$-Trichlorobenzene & 0.50 & $\overline{\mathrm{U}}$ \\
\hline
\end{tabular}


$1 \mathrm{~J}-$ FORM I VOA-TIC

VOLATILE ORGANICS ANALYSIS DATA SHEET

TENTATIVELY IDENTIEIED COMPOUNDS
EPA SAMPLE NO. CNMW06-W-27191

\begin{abstract}
Lab Name: TESTAMERICA BURIINGTON
Lab Code: STLV Case No.: CENTRA

Matrix: (SOIL/SED/WATER) Water

Sample wt/vol: 25.0 $(\mathrm{g} / \mathrm{mL}) \mathrm{mL}$

Level: (TRACE or LOW/MED) TRACE

: Moisture: not dec.

GC Column: DB-624

Soil Extract Volume: ID: $0.20 \quad(\mathrm{~mm})$ (uL)

CONCENTRATION UNITS: (ug/L or $\mathrm{ug} / \mathrm{kg}$ ) $\mathrm{ug} / \mathrm{L}$
\end{abstract}

Contract: $8 \mathrm{E}-00302$

SDG No.: 200-1629

Lab Sample ID: 200-1629-3

Lab File ID: JBMD22.D

Date Received: 09/21/2010

Date Analyzed: 09/24/2010

Dilution Factor: 1.0

Soil Aliquot Volume: (uL)

Purge Volume: 25.0 $(\mathrm{mL})$

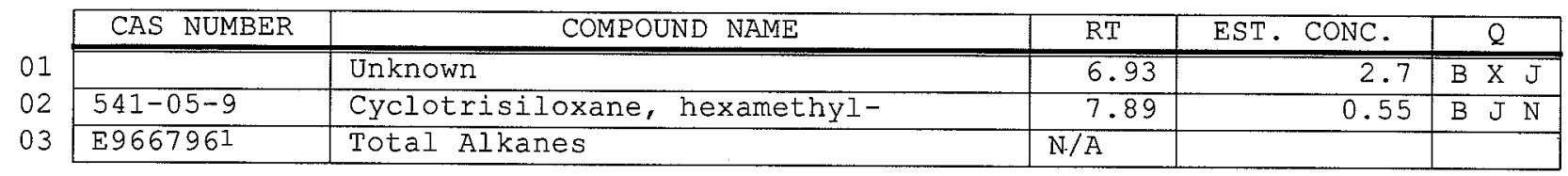

IEPA-designated Registry Number. 
Lab Name: TESTAMERICA BURLINGTON

Lab Code: STLV Case No.: CENTRA Mod. Ref No.:

Matrix: (SOIL/SED/WATER) water

Sample wt/vol: 25.0 $(\mathrm{g} / \mathrm{mL}) \mathrm{mL}$

Level: (TRACE/LOW/MED) TRACE

\% Moisture: not dec.

GC Column: DB-624 ID $: 0.20$ (mm)

Soil Extract Volume: (uL)

Purge Volume: 25.0 $(\mathrm{mL})$
Contract: $8 \mathrm{E}-00302$

SDG No.: 200-1629

\begin{tabular}{|c|c|c|c|}
\hline CAS NO. & COMPOUND & $\begin{array}{l}\text { CONCENTRATION UNITS: } \\
(\mathrm{ug} / \mathrm{I} \text { or } \mathrm{ug} / \mathrm{kg}) \mathrm{ug} / \mathrm{L}\end{array}$ & $Q$ \\
\hline $75-71-8$ & Dichlorodifluoromethane & 0.50 & $\overline{\mathrm{U}}$ \\
\hline $74-87-3$ & Chloromethane & 0.50 & $\mathrm{U}$ \\
\hline $75-01-4$ & Vinyl chloride & 0.50 & $\mathrm{U}$ \\
\hline $74-83-9$ & Bromomethane & 0.50 & $\mathrm{U}$ \\
\hline $75-00-3$ & Chloroethane & 0.50 & $\mathrm{U}$ \\
\hline $75-69-4$ & Trichlorofluoromethane & 0.50 & $\mathrm{U}$ \\
\hline $75-35-4$ & 1,1-Dichloroethene & 0.50 & $\mathrm{U}$ \\
\hline $76-13-1$ & 1,1,2-Trichloro-1,2,2-trifluoroethane & 0.50 & $\mathrm{U}$ \\
\hline $67-64-1$ & Acetone & 2.3 & $\mathrm{JB}$ \\
\hline $75-15-0$ & Carbon disulfide & 0.50 & $\mathrm{U}$ \\
\hline $79-20-9$ & Methyl acetate & 0.50 & $\mathrm{U}$ \\
\hline $75-09-2$ & Methylene Chloride & 0.50 & $\mathrm{U}$ \\
\hline $156-60-5$ & trans-1,2-Dichloroethene & 0.50 & $\overline{\mathrm{U}}$ \\
\hline $1634-04-4$ & Methyl tert-butyl ether & 0.50 & $\bar{U}$ \\
\hline $75-34-3$ & 1,1-Dichloroethane & 0.50 & $\mathrm{U}$ \\
\hline $156-59-2$ & cis-1,2-Dichloroethene & 0.50 & $\mathrm{U}$ \\
\hline $78-93-3$ & 2-Butanone & 5.0 & $\mathrm{U}$ \\
\hline $74-97-5$ & Bromochloromethane & 0.50 & $\mathrm{U}$ \\
\hline $67-66-3$ & Chloroform & 0.50 & $\mathrm{U}$ \\
\hline $71-55-6$ & 1,1,1-Trichloroethane & 0.50 & $\overline{\mathrm{U}}$ \\
\hline $110-82-7$ & Cyclohexane & 0.50 & $\mathrm{U}$ \\
\hline $56-23-5$ & Carbon tetrachloride & 5.2 & \\
\hline $71-43-2$ & Benzene & 0.50 & $\mathrm{U}$ \\
\hline $107-06-2$ & 1,2-Dichloroethane & 0.50 & $\mathrm{U}$ \\
\hline
\end{tabular}

Report 1,4-Dioxane for Low-Medium VOA analysis only

Lab Sample ID: 200-1629-4

Lab File ID: JBMD23.D

Date Received: 09/21/2010

Date Analyzed: 09/24/2010

Dilution Factor: 1.0

Soil Aliquot Volume: (uL) 
Lab Name: TESTAMERICA BURLINGTON

Lab Code: STLV Case No.: CENTRA Mod. Ref No.:

Matrix: (SOIL/SED/WATER) water

Sample wt/vol: 25.0 $(\mathrm{g} / \mathrm{mL}) \mathrm{mL}$

Level: (TRACE/LOW/MED) TRACE

\% Moisture: not dec.

GC Column: DB-624 ID: 0.20 (mm)

Soil Extract Volume: (uL) (mL)

Purge Volume: 25.0

Contract: $8 \mathrm{E}-00302$

SDG No.: 200-1629

Lab Sample ID: 200-1629-4

Lab File ID: JBMD23.D

Date Received: 09/21/2010

Date Analyzed: 09/24/2010

Dilution Factor: 1.0

Soil Aliquot Volume:

(uL)

\begin{tabular}{|c|c|c|c|}
\hline CAS NO. & COMPOUND & $\begin{array}{l}\text { CONCENTRATION UNITS: } \\
(\mathrm{ug} / \mathrm{L} \text { or } \mathrm{ug} / \mathrm{kg}) \mathrm{ug} / \mathrm{L}\end{array}$ & $Q$ \\
\hline $79-01-6$ & Trichloroethene & 0.50 & $\overline{U U}$ \\
\hline $108-87-2$ & Methylcyclohexane & 0.50 & $\bar{U}$ \\
\hline $78-87-5$ & 1,2-Dichloropropane & 0.50 & $\bar{U}$ \\
\hline $75-27-4$ & Bromodichloromethane & 0.50 & $\bar{U}$ \\
\hline $10061-01-5$ & cis-1,3-Dichloropropene & 0.50 & $\mathrm{U}$ \\
\hline $108-10-1$ & 4-Methyl-2-pentanone & 5.0 & $\mathrm{U}$ \\
\hline $108-88-3$ & Toluene & 0.50 & $\mathrm{U}$ \\
\hline $10061-02-6$ & trans-1,3-Dichloropropene & 0.50 & $\mathrm{U}$ \\
\hline $79-00-5$ & $1,1,2$-Trichloroethane & 0.50 & $\mathrm{U}$ \\
\hline $127-18-4$ & Tetrachloroethene & 0.50 & $\mathrm{U}$ \\
\hline $591-78-6$ & 2-Hexanone & 5.0 & $\mathrm{U}$ \\
\hline $124-48-1$ & Dibromochloromethane & 0.50 & $\mathrm{U}$ \\
\hline $106-93-4$ & 1,2-Dibromoethane & 0.50 & $\mathrm{U}$ \\
\hline $108-90-7$ & Chlorobenzene & 0.50 & $\mathrm{U}$ \\
\hline $100-41-4$ & Ethylbenzene & 0.50 & $\mathrm{U}$ \\
\hline $95-47-6$ & o-xylene & 0.50 & $\mathrm{U}$ \\
\hline $179601-23-1$ & m,p-xylene & 0.50 & $\mathrm{U}$ \\
\hline $100-42-5$ & styrene & 0.50 & $\mathrm{U}$ \\
\hline $75-25-2$ & Bromoform & 0.50 & $\mathrm{U}$ \\
\hline $98-82-8$ & Isopropylbenzene & 0.50 & $\mathrm{U}$ \\
\hline $79-34-5$ & $1,1,2,2$-Tetrachloroethane & 0.50 & $\mathrm{U}$ \\
\hline $541-73-1$ & 1,3-Dichlorobenzene & 0.50 & $\mathrm{U}$ \\
\hline $106-46-7$ & 1,4-Dichlorobenzene & 0.50 & $\mathrm{U}$ \\
\hline $95-50-1$ & 1,2-Dichlorobenzene & 0.50 & $\mathrm{U}$ \\
\hline $96-12-8$ & 1,2-Dibromo-3-Chloropropane & 0.50 & $\mathrm{U}$ \\
\hline $120-82-1$ & 1,2,4-Trichlorobenzene & 0.50 & $\mathrm{U}$ \\
\hline $87-61-6$ & 1,2,3-Trichlorobenzene & 0.50 & $\bar{U}$ \\
\hline
\end{tabular}




\section{$1 \mathrm{~J}$ - FORM I VOA-TIC \\ VOLATILE ORGANICS ANALYSIS DATA SHEET \\ TENTATIVELY IDENTIEIED COMPOUNDS}

EPA SAMPLE NO.

CNMW07-W-27192

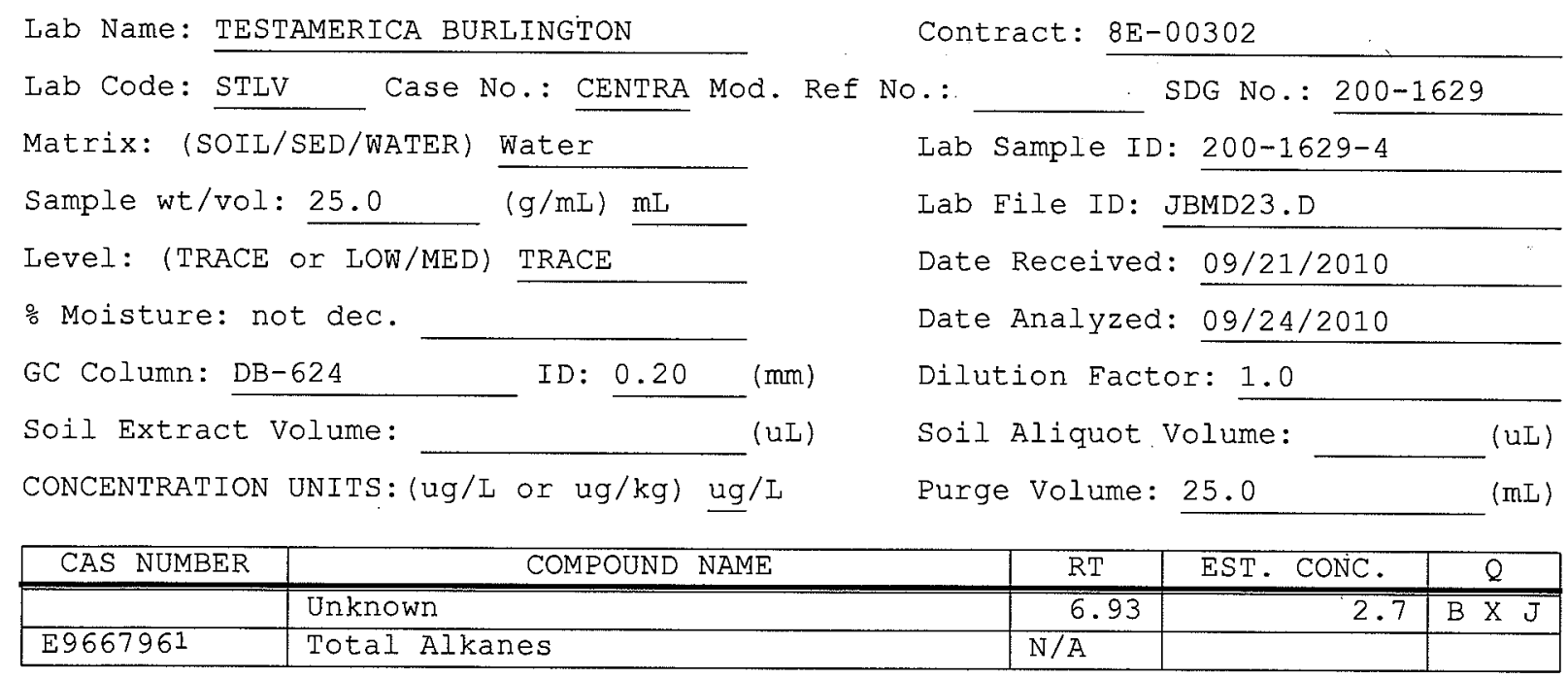

1 EPA-designated Registry Number. 
Lab Name: TESTAMERICA BURLINGTON

Lab Code: STLV Case No.: CENTRA Mod. Ref No.:

Matrix: (SOIL/SED/WATER) Water

Sample wt/vol: 25.0 $(\mathrm{g} / \mathrm{mL}) \mathrm{mL}$

Level: (TRACE/LOW/MED) TRACE

\% Moisture: not dec.

GC Column: DB-624 ID: 0.20

Soil Extract Volume:

Purge Volume: 25.0
Contract: $8 \mathrm{E}-00302$

SDG NO.: 200-1629

\begin{tabular}{|l|l|c|c|}
\hline CAS NO. & COMPOUND & $\begin{array}{c}\text { CONCENTRATION UNITS: } \\
\text { (ug/I or ug/kg) ug/L }\end{array}$ & Q \\
\hline \hline $75-71-8$ & Dichlorodifluoromethane & 0.50 & $\mathrm{U}$ \\
\hline $74-87-3$ & Chloromethane & 0.50 & $\mathrm{U}$ \\
\hline $75-01-4$ & Vinyl chloride & 0.50 & $\mathrm{U}$ \\
\hline $74-83-9$ & Bromomethane & 0.50 & $\mathrm{U}$ \\
\hline $75-00-3$ & Chloroethane & 0.50 & $\mathrm{U}$ \\
\hline $75-69-4$ & Trichlorofluoromethane & 0.50 & $\mathrm{U}$ \\
\hline $75-35-4$ & $1,1-$ Dichloroethene & 0.50 & $\mathrm{U}$ \\
\hline $76-13-1$ & $1,1,2-$ Trichloro-1,2,2-trifluoroethane & 0.50 & $\mathrm{U}$ \\
\hline $67-64-1$ & Acetone & 2.8 & $\mathrm{~J}$ \\
\hline $75-15-0$ & Carbon disulfide & 0.50 \\
\hline $79-20-9$ & Methyl acetate & $\mathrm{U}$ \\
\hline $75-09-2$ & Methylene Chloride & 0.50 & $\mathrm{U}$ \\
\hline $156-60-5$ & trans-1,2-Dichloroethene & 0.50 & $\mathrm{U}$ \\
\hline $1634-04-4$ & Methyl tert-butyl ether & 0.50 & $\mathrm{U}$ \\
\hline $75-34-3$ & $1,1-$ Dichloroethane & 0.50 & $\mathrm{U}$ \\
\hline $156-59-2$ & Cis-1,2-Dichloroethene & 0.50 & $\mathrm{U}$ \\
\hline $78-93-3$ & 2-Butanone & 0.50 & $\mathrm{U}$ \\
\hline $74-97-5$ & Bromochloromethane & 5.0 & $\mathrm{U}$ \\
\hline $67-66-3$ & Chloroform & 0.50 & $\mathrm{U}$ \\
\hline $71-55-6$ & 1,1,1-Trichloroethane & 0.50 & $\mathrm{U}$ \\
\hline $110-82-7$ & Cyclohexane & 0.50 & $\mathrm{U}$ \\
\hline $56-23-5$ & Carbon tetrachloride & 0.50 & $\mathrm{U}$ \\
\hline $71-43-2$ & Benzene & 0.50 & $\mathrm{U}$ \\
\hline $107-06-2$ & $1,2-$ Dichloroethane & 0.50 & $\mathrm{U}$ \\
\hline
\end{tabular}

Report 1,4-Dioxane for Low-Medium VOA analysis only

Lab Sample ID: 200-1629-1

Lab File ID: JBMD20.D

Date Received: 09/21/2010

Date Analyzed: 09/23/2010

Dilution Factor: 1.0

Soil Aliquot Volume: (uL) (uL) (mL) 
Lab Name: TESTAMERICA BURLINGTON

Lab Code: STLV

Matrix: (SOIL/SED/WATER)

Sample wt/vol: 25.0

Water

$(\mathrm{g} / \mathrm{mL}) \mathrm{mI}$

Level: (TRACE/LOW/MED) TRACE

\% Moisture: not dec.

GC Column: $\mathrm{DB}-624$

Soil Extract volume:

ID: $0.20 \quad(\mathrm{~mm})$

(UL)

(mL)

Purge Volume: 25.0

Contract: $8 \mathrm{E}-00302$

SDG No.: 200-1629

Lab Sample ID: 200-1629-1

Lab File ID: JBMD20.D

Date Received: 09/21/2010

Date Analyzed: 09/23/2010

Dilution Factor: 1.0

Soil Aliquot Volume:

(UI)

\begin{tabular}{|c|c|c|c|}
\hline CAS NO. & COMPOUND & $\begin{array}{l}\text { CONCENTRATION UNITS: } \\
(\mathrm{ug} / \mathrm{L} \text { or } \mathrm{ug} / \mathrm{kg}) \mathrm{ug} / \mathrm{L} \\
\end{array}$ & $\dot{Q}$ \\
\hline $79-01-6$ & Trichloroethene & 0.50 & $\overline{\mathrm{U}}$ \\
\hline $108-87-2$ & Methylcyclohexane & 0.50 & $\overline{\mathrm{U}}$ \\
\hline $78-87-5$ & 1,2-Dichloropropane & 0.50 & $\overline{\mathrm{U}}$ \\
\hline $75-27-4$ & Bromodichloromethane & 0.50 & $\overline{\mathrm{U}}$ \\
\hline $10061-01-5$ & cis-1,3-Dichloropropene & 0.50 & $\mathrm{U}$ \\
\hline $108-10-1$ & 4-Methyl-2-pentanone & 5.0 & $\mathrm{U}$ \\
\hline $108-88-3$ & Toluene & 0.50 & $\bar{U}$ \\
\hline $10061-02-6$ & trans-1,3-Dichloropropene & 0.50 & $\overline{\mathrm{U}}$ \\
\hline $79-00-5$ & 1,1,2-Trichloroethane & 0.50 & $\mathrm{U}$ \\
\hline $127-18-4$ & Tetrachloroethene & 0.50 & $\bar{U}$ \\
\hline $591-78-6$ & 2-Hexanone & 5.0 & $\mathrm{U}$ \\
\hline $124-48-1$ & Dibromochloromethane & 0.50 & $\mathrm{U}$ \\
\hline $106-93-4$ & 1,2-Dibromoethane & 0.50 & $\mathrm{U}$ \\
\hline $108-90-7$ & Chlorobenzene & 0.50 & $\mathrm{U}$ \\
\hline $100-41-4$ & Ethylbenzene & 0.50 & $\mathrm{U}$ \\
\hline $95-47-6$ & o-Xylene & 0.50 & $\mathrm{U}$ \\
\hline $179601-23-1$ & m,p-xylene & 0.50 & $\mathrm{U}$ \\
\hline $100-42-5$ & Styrene & 0.50 & $\mathrm{U}$ \\
\hline $75-25-2$ & Bromoform & 0.50 & $\mathrm{U}$ \\
\hline $98-82-8$ & Isopropylbenzene & 0.50 & $\mathrm{U}$ \\
\hline $79-34-5$ & $1,1,2,2$-Tetrachloroethane & 0.50 & $\mathrm{U}$ \\
\hline $541-73-1$ & 1,3-Dichlorobenzene & 0.50 & $\mathrm{U}$ \\
\hline $106-46-7$ & 1,4-Dichlorobenzene & 0.50 & $\mathrm{U}$ \\
\hline $95-50-1$ & 1,2-Dichlorobenzene & 0.50 & $\mathrm{U}$ \\
\hline $96-12-8$ & 1,2-Dibromo-3-Chloropropane & 0.50 & $\mathrm{U}$ \\
\hline $120-82-1$ & $1,2,4$-Trichlorobenzene & 0.50 & $\mathrm{U}$ \\
\hline $87-61-6$ & 1,2,3-Trichlorobenzene & 0.50 & $\mathrm{U}$ \\
\hline
\end{tabular}


$1 \mathrm{~J}$ - FORM I VOA-TIC

VOLATILE ORGANICS ANALYSIS DATA SHEET

TENTATIVELY IDENTIFIED COMPOUNDS
EPA' SAMPLE NO.

CNMW08-S-27193
Lab Name: TESTAMERICA BURLINGTON Case No.: CENTRA Mod. Ref No.:

Contract: $8 E-00302$

Lab Code: STLV

Matrix: (SOIL/SED/WATER) Water

Sample wt/vol: 25.0 $(\mathrm{g} / \mathrm{mL}) \mathrm{mL}$

Level: (TRACE or LOW/MED) TRACE

\% Moisture: not dec.

GC Column: DB-624 ID: 0.20 (mm)

Soil Extract Volume: (uI)

CONCENTRATION UNITS: (ug/L Or $\mathrm{ug} / \mathrm{kg}$ ) $\mathrm{ug} / \mathrm{L}$
SDG No.: 200-1629

Lab Sample ID: 200-1629-1

Lab File ID: JBMD20.D

Date Received: 09/21/2010

Date Analyzed: 09/23/2010

Dilution Factor: 1.0

Soil Aliquot Volume: (uI)

Purge Volume: 25.0 (mI)

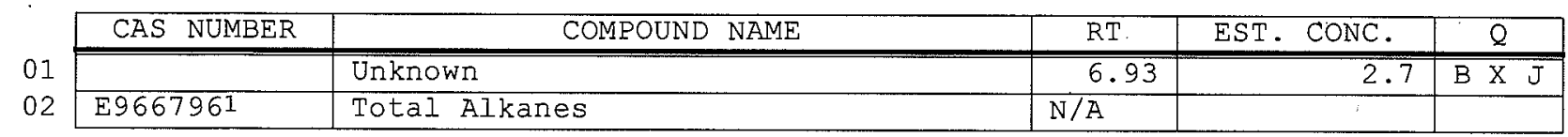

1EPA-designated Registry Number. 
Lab Name: TESTAMERICA BURLINGTON

Lab Code: STIV

Matrix: (SOII/SED/WATER)

Sample wt/vol: 25.0

Case No.: CENTRA Mod. Ref No.:
Contract: $8 \mathrm{E}-00302$

\section{Lab Sample ID: 200-1629-5}

Lab File ID: JBMD24.D

Date Received: 09/21/2010

Date Analyzed: 09/24/2010

Dilution Factor: 1.0

Soil Aliquot volume: $\left(U I_{1}\right)$ (mL)

\begin{tabular}{|c|c|c|c|}
\hline CAS NO. & COMPOUND & $\begin{array}{l}\text { CONCENTRATION UNITS: } \\
(\mathrm{ug} / \mathrm{I} \text { or } u g / \mathrm{kg}) \mathrm{ug} / \mathrm{L}\end{array}$ & $Q$ \\
\hline $75-71-8$ & Dichlorodifluoromethane & 0.50 & $\overline{\mathrm{U}}$ \\
\hline $74-87-3$ & Chloromethane & 0.50 & $\mathrm{U}$ \\
\hline $75-01-4$ & Vinyl chloride & 0.50 & $\mathrm{U}$ \\
\hline $74-83-9$ & Bromomethane & 0.50 & $\mathrm{U}$ \\
\hline $75-00-3$ & Chloroethane & 0.50 & U \\
\hline $75-69-4$ & Trichlorofluoromethane & 0.50 & $\mathrm{U}$ \\
\hline $75-35-4$ & 1,1-Dichloroethene & 0.50 & $\mathrm{U}$ \\
\hline $76-13-1$ & $1,1,2-\operatorname{Trichloro-1,2,2-trifluoroethane}$ & 0.50 & $\mathrm{U}$ \\
\hline $67-64-1$ & Acetone & 6.4 & $\bar{B}$ \\
\hline $75-15-0$ & Carbon disulfide & 0.50 & $\mathrm{U}$ \\
\hline $79-20-9$ & Methyl acetate & 0.50 & $\mathrm{U}$ \\
\hline $75-09-2$ & Methylene Chloride & 0.50 & $\mathrm{U}$ \\
\hline $156-60-5$ & trans-1,2-Dichloroethene & 0.50 & $\mathrm{U}$ \\
\hline $1634-04-4$ & Methyl tert-butyl ether & 0.50 & $\mathrm{U}$ \\
\hline $75-34-3$ & 1,1-Dichloroethane & 0.50 & $\mathrm{U}$ \\
\hline $156-59-2$ & cis-1,2-Dichloroethene & 0.50 & U \\
\hline $78-93-3$ & 2-Butanone & 5.0 & U \\
\hline $74-97-5$ & Bromochloromethane & 0.50 & $\mathrm{U}$ \\
\hline $67-66-3$ & Chloroform & 0.20 & $\mathrm{~J}$ \\
\hline $71-55-6$ & $1,1,1$-Trichloroethane & 0.50 & $\mathrm{U}$ \\
\hline $110-82-7$ & Cyclohexane & 0.50 & U \\
\hline $56-23-5$ & Carbon tetrachloride & 0.50 & $\overline{\mathrm{U}}$ \\
\hline $71-43-2$ & Benzene & 0.50 & $\bar{U}$ \\
\hline $107-06-2$ & 1,2-Dichloroethane & 0.50 & $\mathrm{U}$ \\
\hline
\end{tabular}

Report 1,4-Dioxane for Low-Medium VOA analysis only 
Lab Name: TESTAMERICA BURLINGTON

Lab Code: STLV Case No.: CENTRA Mod. Ref No.:
Contract: $8 \mathrm{E}-00302$ SDG No.: 200-1629
Matrix: (SOIL/SED/WATER) Water

Sample wt/vol: 25.0 $(\mathrm{g} / \mathrm{mL}) \mathrm{mL}$

Level: (TRACE/LOW/MED) TRACE

\% Moisture: not dec.

GC Column: DB-624 ID: $0.20 \quad(\mathrm{~mm})$

Soil Extract volume: (uL) (mL)

Purge Volume: 25.0
Lab Sample ID: 200-1629-5

Lab File ID: JBMD24.D

Date Received: 09/21/2010

Date Analyzed: 09/24/2010

Dilution Factor: 1.0

Soil Aliquot Volume: (uL)

\begin{tabular}{|c|c|c|c|}
\hline CAS NO. & COMPOUND & $\begin{array}{l}\text { CONCENTRATION UNITS: } \\
(\mathrm{ug} / \mathrm{L} \text { or } \mathrm{ug} / \mathrm{kg}) \mathrm{ug} / \mathrm{L} \\
\end{array}$ & $Q$ \\
\hline $79-01-6$ & Trichloroethene & 0.50 & $\overline{\mathrm{U}}$ \\
\hline $108-87-2$ & Methylcyclohexane & 0.50 & $\mathrm{U}$ \\
\hline $78-87-5$ & 1,2-Dichloropropane & 0.50 & $\mathrm{U}$ \\
\hline $75-27-4$ & Bromodichloromethane & 0.15 & $\mathrm{~J}$ \\
\hline $10061-01-5$ & cis-1,3-Dichloropropene & 0.50 & $\mathrm{U}$ \\
\hline $108-10-1$ & 4-Methyl-2-pentanone & 5.0 & $\mathrm{U}$ \\
\hline $108-88-3$ & Toluene & 0.099 & $\mathrm{~J}$ \\
\hline $10061-02-6$ & trans-1,3-Dichloropropene & 0.50 & $\mathrm{U}$ \\
\hline $79-00-5$ & $1,1,2-$ Trichloroethane & 0.50 & $\mathrm{U}$ \\
\hline $127-18-4$ & Tetrachloroethene & 0.50 & $\mathrm{U}$ \\
\hline $591-78-6$ & 2-Hexanone & 5.0 & $\mathrm{U}$ \\
\hline $124-48-1$ & Dibromochloromethane & 0.081 & $\mathrm{~J}$ \\
\hline $106-93-4$ & 1,2-Dibromoethane & 0.50 & $\mathrm{U}$ \\
\hline $108-90-7$ & Chlorobenzene & 0.50 & $\mathrm{U}$ \\
\hline $100-41-4$ & Ethylbenzene & 0.50 & $\mathrm{U}$ \\
\hline $95-47-6$ & o-Xylene & 0.044 & $\mathrm{~J}$ \\
\hline $179601-23-1$ & $\mathrm{~m}, \mathrm{p}$-Xylene & 0.50 & $\mathrm{U}$ \\
\hline $100-42-5$ & Styrene & 0.50 & $\mathrm{U}$ \\
\hline $75-25-2$ & Bromoform & 0.50 & $\mathrm{U}$ \\
\hline $98-82-8$ & Isopropylbenzene & 0.50 & $\mathrm{U}$ \\
\hline $79-34-5$ & $1,1,2,2$-Tetrachloroethane & 0.50 & $\mathrm{U}$ \\
\hline $541-73-1$ & 1,3-Dichlorobenzene & 0.50 & $\mathrm{U}$ \\
\hline $106-46-7$ & 1,4-Dichlorobenzene & 0.039 & $\mathrm{~J}$ \\
\hline $95-50-1$ & 1,2-Dichlorobenzene & 0.50 & $\mathrm{U}$ \\
\hline $96-12-8$ & 1,2-Dibromo-3-Chloropropane & 0.50 & $\mathrm{U}$ \\
\hline $120-82-1$ & $1,2,4$-Trichlorobenzene & 0.50 & $\mathrm{U}$ \\
\hline $87-61-6$ & 1,2,3-Trichlorobenzene & 0.50 & $\mathrm{U}$ \\
\hline
\end{tabular}


IJ - FORM I VOA-TIC

VOLATILE ORGANICS ANALYSIS DATA SHEET

TENTATIVELY IDENTIFIED COMPOUNDS
EPA SAMPLE NO. CNQCTB-W-27216

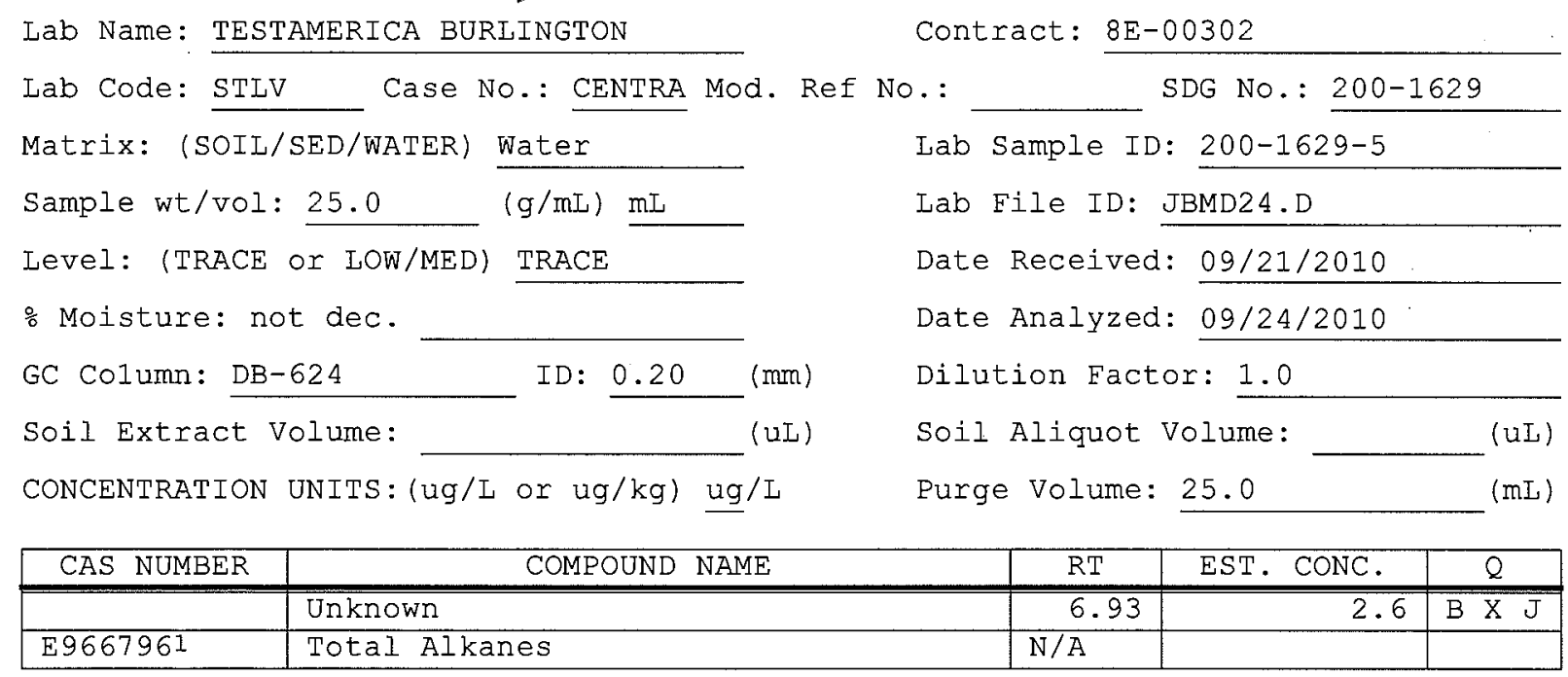

1EPA-designated Registry Number. 
Lab Name: TESTAMERICA BURLINGTON

Lab Code: STLV

Matrix: (SOIL/SED/WATER)

Sample wt/vol: 25.0 Case No.: CENTRA Mod. Ref No.:

Contract: $8 \mathrm{E}-00302$

Level: (TRACE/LOW/MED) TRACE

\% Moisture: not dec.

GC Column: DB-624

ID $: 0.20$
Water

$(\mathrm{g} / \mathrm{mL}) \mathrm{mL}$
Lab Sample ID: 200-1629-2

Lab File ID: JBMD21.D

Date Received: 09/21/2010

Date Analyzed: 09/24/2010

Dilution Factor: 1.0

Soil Aliquot Volume: (uL) (mm)

UL) (mL)

\begin{tabular}{|c|c|c|c|}
\hline CAS NO. & COMPOUND & $\begin{array}{l}\text { CONCENTRATION UNITS: } \\
(\mathrm{ug} / \mathrm{L} \text { or } \mathrm{ug} / \mathrm{kg}) \mathrm{ug} / \mathrm{I} \\
\end{array}$ & $Q$ \\
\hline $75-71-8$ & Dichlorodifluoromethane & 0.50 & $\mathrm{U}$ \\
\hline $74-87-3$ & Chloromethane & 0.50 & $\mathrm{U}$ \\
\hline $75-01-4$ & Vinyl chloride & 0.50 & $\mathrm{U}$ \\
\hline $74-83-9$ & Bromomethane & 0.50 & $\mathrm{U}$ \\
\hline $75-00-3$ & Chloroethane & 0.50 & $\mathrm{U}$ \\
\hline $75-69-4$ & Trichlorofluoromethane & 0.50 & $\mathrm{U}$ \\
\hline $75-35-4$ & 1,1-Dichloroethene & 0.50 & $\mathrm{U}$ \\
\hline $76-13-1$ & 1,1,2-Trichloro-1,2,2-trifluoroethane & 0.50 & $\mathrm{U}$ \\
\hline $67-64-1$ & Acetone & 2.1 & $\mathrm{~J} B$ \\
\hline $75-15-0$ & Carbon disulfide & 0.50 & $\mathrm{U}$ \\
\hline $79-20-9$ & Methyl acetate & 0.50 & $\mathrm{U}$ \\
\hline $75-09-2$ & Methylene Chloride & 0.50 & $\mathrm{U}$ \\
\hline $156-60-5$ & trans-1,2-Dichloroethene & 0.50 & $\mathrm{U}$ \\
\hline $1634-04-4$ & Methyl tert-butyl ether & 0.50 & $\mathrm{U}$ \\
\hline $75-34-3$ & 1,1-Dichloroethane & 0.50 & $\mathrm{U}$ \\
\hline $156-59-2$ & cis-1,2-Dichloroethene & 0.50 & $\mathrm{U}$ \\
\hline $78-93-3$ & 2-Butanone & 5.0 & $\mathrm{U}$ \\
\hline $74-97-5$ & Bromochloromethane & 0.50 & $\mathrm{U}$ \\
\hline $67-66-3$ & Chloroform & 0.50 & $\mathrm{U}$ \\
\hline $71-55-6$ & 1,1,1-Trichloroethane & 0.50 & $\mathrm{U}$ \\
\hline $110-82-7$ & Cyclohexane & 0.50 & $\mathrm{U}$ \\
\hline $56-23-5$ & Carbon tetrachloride & 0.50 & $\mathrm{U}$ \\
\hline $71-43-2$ & Benzene & 0.50 & $\mathrm{U}$ \\
\hline $107-06-2$ & 1,2-Dichloroethane & 0.50 & $\mathrm{U}$ \\
\hline
\end{tabular}

Report 1,4-Dioxane for Low-Medium VOA analysis only 
Lab Name: TESTAMERICA BURLINGTON

Lab Code: STLV

Matrix: (SOIL/SED/WATER)

Sample wt/vol: 25.0 Case No.: CENTRA Mod. Ref No.:

Contract: $8 \mathrm{E}-00302$

Level: (TRACE/LOW/MED) TRACE

\% Moisture: not dec.

GC Column: $\mathrm{DB}-624$

ID: $0.20 \quad(\mathrm{~mm})$

Soil Extract volume: (uL)

Purge Volume: 25.0 (mL)
SDG No.: 200-1629

Lab Sample ID: 200-1629-2

Lab File ID: JBMD21.D

Date Received: 09/21/2010

Date Analyzed: 09/24/2010

Dilution Factor: 1.0

Soil Aliquot volume: (uL)

\begin{tabular}{|c|c|c|c|}
\hline CAS NO. & COMPOUND & $\begin{array}{l}\text { CONCENTRATION UNITS: } \\
(u g / L \text { or } u g / \mathrm{kg}) \mathrm{ug} / \mathrm{L}\end{array}$ & $Q$ \\
\hline $79-01-6$ & Trichloroethene & 0.50 & $\overline{\mathrm{U}}$ \\
\hline $108-87-2$ & Methylcyclohexane & 0.50 & $\mathrm{U}$ \\
\hline $78-87-5$ & 1,2-Dichloropropane & 0.50 & $\mathrm{U}$ \\
\hline $75-27-4$ & Bromodichloromethane & 0.50 & $\mathrm{U}$ \\
\hline $10061-01-5$ & cis-1,3-Dichloropropene & 0.50 & $\mathrm{U}$ \\
\hline $108-10-1$ & 4-Methyl-2-pentanone & 5.0 & $\mathrm{U}$ \\
\hline $108-88-3$ & Toluene & 0.50 & $\mathrm{U}$ \\
\hline $10061-02-6$ & trans-1,3-Dichloropropene & 0.50 & $\bar{U}$ \\
\hline $79-00-5$ & $1,1,2-$ Trichloroethane & 0.50 & $\mathrm{U}$ \\
\hline $127-18-4$ & Tetrachloroethene & 0.50 & $\mathrm{U}$ \\
\hline $591-78-6$ & 2-Hexanone & 5.0 & $\mathrm{U}$ \\
\hline $124-48-1$ & Dibromochloromethane & 0.50 & $\mathrm{U}$ \\
\hline $106-93-4$ & 1,2-Dibromoethane & 0.50 & $\bar{U}$ \\
\hline $108-90-7$ & Chlorobenzene & 0.50 & $\mathrm{U}$ \\
\hline $100-41-4$ & Ethylbenzene & 0.50 & $\mathrm{U}$ \\
\hline $95-47-6$ & o-Xylene & 0.50 & $\mathrm{U}$ \\
\hline $179601-23-1$ & m,p-xylene & 0.50 & $\mathrm{U}$ \\
\hline $100-42-5$ & styrene & 0.50 & $\mathrm{U}$ \\
\hline $75-25-2$ & Bromoform & 0.50 & $\mathrm{U}$ \\
\hline $98-82-8$ & Isopropylbenzene & 0.50 & $\mathrm{U}$ \\
\hline $79-34-5$ & $1,1,2,2$-Tetrachloroethane & 0.50 & $\mathrm{U}$ \\
\hline $541-73-1$ & 1,3-Dichlorobenzene & 0.50 & $\mathrm{U}$ \\
\hline $106-46-7$ & 1,4-Dichlorobenzene & 0.50 & $\mathrm{U}$ \\
\hline $95-50-1$ & 1,2-Dichlorobenzene & 0.50 & $\mathrm{U}$ \\
\hline $96-12-8$ & 1,2-Dibromo-3-Chloropropane & 0.50 & $\mathrm{U}$ \\
\hline $120-82-1$ & $1,2,4$-Trichlorobenzene & 0.50 & $\mathrm{U}$ \\
\hline $87-61-6$ & 1,2,3-Trichlorobenzene & 0.50 & $\mathrm{U}$ \\
\hline
\end{tabular}




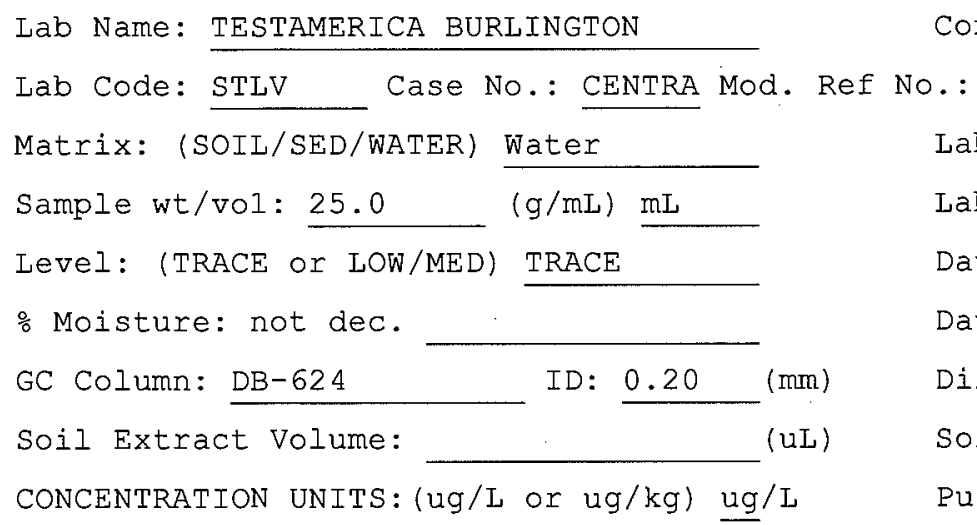

Matrix: (SOIL/SED/WATER) Water

Sample wt/vol: 25.0 $(\mathrm{g} / \mathrm{mL}) \mathrm{mL}$

Level: (TRACE or LOW/MED) TRACE

\% Moisture: not dec.

GC Column: DB-624 ID: 0.20 $(\mathrm{mm})$

Soil Extract Volume: (uL) CONCENTRATION UNITS: (ug/L or $\mathrm{ug} / \mathrm{kg}$ ) $\mathrm{ug} / \mathrm{L}$

Lab Sample ID: 200-1629-2

Lab File ID: JBMD21.D

Date Received: 09/21/2010

Date Analyzed: 09/24/2010

Dilution Factor: 1.0

Soil Aliquot Volume: (uL)

Purge Volume: 25.0 (mL)

\begin{tabular}{|c|c|c|c|c|c|}
\hline & CAS NUMBER & COMPOUND NAME & RT & EST. CONC. & $Q$ \\
\hline 01 & & Unknown & 6.93 & 2.7 & $\mathrm{~B} \times \mathrm{J}$ \\
\hline 02 & $541-05-9$ & Cyclotrisiloxane, hexamethyl- & 7.89 & 0.60 & $\mathrm{~B} J \mathrm{~N}$ \\
\hline 03 & E9667961 & Total Alkanes & $\mathrm{N} / \mathrm{A}$ & & \\
\hline
\end{tabular}


$6 \mathrm{~A}-$ FORM VI $\mathrm{VOA}-1$

VOLATILE ORGANICS INITIAL CALIBRATION DATA

Lab Name: TESTAMERICA BURLINGTON

Contract: $8 \mathrm{E}-00302$

Lab Code: STIV Case No.: CENTRA Mod. Ref No.: SDG No.: 200-1629

Instrument ID: J.i Calibration Date(s): 09/21/2010 09/21/2010

Heated Purge: $(\mathrm{Y} / \mathrm{N}) \mathrm{N}$ Calibration Time(s): 1435 1724

Purge Volume: 25.0

GC Column: DB-624

ID: 0.20 (mL)

(mm) Length: 25

(m)

\begin{tabular}{|c|c|c|c|c|c|c|c|}
\hline LAB FILE ID: & RREO. $=$ & $\mathrm{BMO} 2 . \mathrm{D}$ & & RRE1: & $=J B M$ & $3 . \mathrm{D}$ & \\
\hline $\mathrm{RRF} 5.0=\mathrm{JBM} 04 . \mathrm{D}$ & RRF10 & BM05.D & & RRF20 & $=\mathrm{JBMC}$ & B. D & \\
\hline COMPOUND & RRF 0.5 & RRF1.0 & RRF5. 0 & RRE10 & RRF20 & $\overline{\mathrm{RRF}}$ & $\because \mathrm{RSD}$ \\
\hline Dichlorodifluoromethane & 0.475 & 0.484 & 0.451 & 0.459 & 0.442 & 0.462 & 3.7 \\
\hline Chloromethane & 0.426 & 0.401 & 0.361 & 0.348 & 0.328 & 0.373 & 10.8 \\
\hline Vinyl chloride & 0.389 & 0.404 & 0.370 & 0.371 & 0.352 & 0.377 & 5.3 \\
\hline Bromomethane & 0.228 & 0.221 & 0.198 & 0.200 & 0.203 & 0.210 & 6.5 \\
\hline Chloroethane & 0.166 & 0.150 & 0.225 & 0.233 & 0.213 & 0.198 & 18.7 \\
\hline Trichlorofluoromethane & 0.581 & 0.610 & 0.583 & 0.592 & 0.563 & 0.586 & 2.9 \\
\hline 1,1-Dichloroethene & 0.289 & 0.290 & 0.279 & 0.287 & 0.280 & 0.285 & 1.8 \\
\hline $\begin{array}{l}\text { 1,1,2-Trichloro- } \\
1,2,2 \text {-trifluoroethane }\end{array}$ & 0.327 & 0.337 & 0.315 & 0.325 & 0.326 & 0.326 & 2.3 \\
\hline Acetone & 0.016 & 0.016 & 0.011 & 0.011 & 0.011 & 0.013 & 21.7 \\
\hline Carbon disulfide & 0.861 & 0.829 & 0.753 & 0.773 & 0.750 & 0.793 & 6.2 \\
\hline Methyl acetate & 0.033 & 0.044 & 0.035 & 0.035 & 0.035 & 0.037 & 12.0 \\
\hline Methylene Chloride & 0.211 & 0.211 & 0.215 & 0.217 & 0.209 & 0.213 & 1.6 \\
\hline trans-1,2-Dichloroethene & 0.305 & 0.311 & 0.297 & 0.305 & 0.297 & 0.303 & 2.0 \\
\hline Methyl tert-butyl ether & 0.311 & 0.336 & 0.321 & 0.333 & 0.323 & 0.325 & 3.1 \\
\hline 1,1-Dichloroethane & 0.480 & 0.528 & 0.487 & 0.502 & 0.481 & 0.496 & 4.0 \\
\hline Cis-1,2-Dichloroethene & 0.286 & 0.295 & 0.286 & 0.291 & 0.283 & 0.288 & 1.7 \\
\hline 2-Butanone & 0.018 & 0.020 & 0.019 & 0.019 & 0.019 & 0.019 & 4.1 \\
\hline Bromochloromethane & 0.084 & 0.091 & 0.086 & 0.085 & 0.084 & 0.086 & 3.4 \\
\hline Chloroform & 0.474 & 0.506 & 0.467 & 0.481 & 0.468 & 0.479 & 3.4 \\
\hline 1,1,1-Trichloroethane & 0.662 & 0.692 & 0.668 & 0.689 & 0.643 & 0.671 & 3.0 \\
\hline Cyclohexane & 0.654 & 0.674 & 0.663 & 0.679 & 0.636 & 0.661 & 2.6 \\
\hline Carbon tetrachloride & 0.589 & 0.622 & 0.610 & 0.629 & 0.599 & 0.610 & 2.7 \\
\hline Benzene & 1.492 & 1.607 & 1.562 & 1.599 & 1.514 & 1.555 & 3.3 \\
\hline 1,2-Dichloroethane & 0.192 & 0.196 & 0.190 & 0.201 & 0.192 & 0.194 & 2.2 \\
\hline Trichloroethene & 0.423 & 0.428 & 0.417 & 0.428 & 0.399 & 0.419 & 2.9 \\
\hline Methylcyclohexane & 0.517 & 0.551 & 0.536 & 0.539 & 0.515 & 0.532 & 2.9 \\
\hline
\end{tabular}

Report 1,4-Dioxane for Low-Medium VOA analysis only 
$6 \mathrm{~B}$ - FORM VI VOA-2

VOLATILE ORGANICS INITIAL CALIBRATION DATA

Lab Name: TESTAMERICA BURLINGTON

Lab Code: STLV

Instrument ID: J.i

Heated Purge: ( $\mathrm{Y} / \mathrm{N}) \mathrm{N}$

Purge Volume: 25.0

GC Column: DB-624
Case No.: CENTRA Mod. Ref No.:

Contract: $8 \mathrm{E}-00302$ Calibration Date(s): 09/21/2010 09/21/2010 Calibration Time(s): 1435 1724 (mL)

\begin{tabular}{|c|c|c|c|c|c|c|c|}
\hline LAB FILE ID: & \multirow{2}{*}{\multicolumn{3}{|c|}{$\begin{array}{l}\text { RRF0.5 }=\text { JBM02.D } \\
\text { RRF10 }=\text { JBM05.D }\end{array}$}} & \multicolumn{4}{|c|}{ RRF1.0 = JBM03.D } \\
\hline RRF5.0 = JBM0 4.D & & & & RRF2 & $=\mathrm{JBMC}$ & 3. D & \\
\hline COMPOUND & RRE0. 5 & RRF1. 0 & RRF5. 0 & RRE 10 & RRE20 & $\overline{\mathrm{RRF}}$ & $\because \mathrm{RSD}$ \\
\hline 1,2-Dichloropropane & 0.262 & 0.296 & 0.287 & 0.301 & 0.282 & 0.286 & 5.2 \\
\hline Bromodichloromethane & 0.314 & 0.351 & 0.353 & 0.353 & 0.344 & 0.343 & 4.8 \\
\hline cis-1,3-Dichloropropene & 0.394 & 0.406 & 0.418 & 0.433 & 0.420 & 0.414 & 3.5 \\
\hline 4-Methyl-2-pentanone & 0.056 & 0.064 & 0.063 & 0.066 & 0.062 & 0.062 & 6.4 \\
\hline Toluene & 1.605 & 1.697 & 1.663 & 1.702 & 1.646 & 1.663 & 2.4 \\
\hline trans-1,3-Dichloropropene & 0.256 & 0.287 & 0.295 & 0.306 & 0.297 & 0.288 & 6.7 \\
\hline 1,1,2-Trichloroethane & 0.117 & 0.148 & 0.145 & 0.145 & 0.133 & 0.138 & 9.3 \\
\hline Tetrachloroethene & 0.368 & 0.381 & 0.378 & 0.384 & 0.365 & 0.375 & 2.2 \\
\hline 2-Hexanone & 0.051 & 0.048 & 0.043 & 0.044 & 0.041 & 0.045 & 9.7 \\
\hline Dibromochloromethane & 0.153 & 0.178 & 0.183 & 0.183 & 0.186 & 0.177 & 7.7 \\
\hline 1,2-Dibromoethane & 0.120 & 0.128 & 0.132 & 0.135 & 0.124 & 0.128 & 4.6 \\
\hline Chlorobenzene & 0.963 & 1.022 & 0.976 & 1.005 & 0.964 & 0.986 & 2.7 \\
\hline Ethylbenzene & 1.835 & 1.939 & 1.953 & 2.017 & 1.966 & 1.942 & 3.4 \\
\hline o-xylene & 0.623 & 0.659 & 0.679 & 0.700 & 0.676 & 0.667 & 4.4 \\
\hline $\mathrm{m}, \mathrm{p}$-Xylene & 0.704 & 0.742 & 0.750 & 0.782 & 0.752 & 0.746 & 3.8 \\
\hline Styrene & 0.843 & 0.908 & 0.965 & 1.030 & 1.002 & 0.950 & 7.9 \\
\hline Bromoform & 0.174 & 0.161 & 0.181 & 0.165 & 0.181 & 0.172 & 5.3 \\
\hline Isopropylbenzene & 1.847 & 1.942 & 1.997 & 2.070 & 2.014 & 1.974 & 4.3 \\
\hline $1,1,2,2$-Tetrachloroethane & 0.114 & 0.124 & 0.126 & 0.126 & 0.120 & 0.122 & 4.3 \\
\hline 1,3-Dichlorobenzene & 1.498 & 1.516 & 1.496 & 1.507 & 1.482 & 1.500 & 0.9 \\
\hline 1,4-Dichlorobenzene & 1.507 & 1.528 & 1.457 & 1.468 & 1.422 & 1.476 & 2.8 \\
\hline 1,2-Dichlorobenzene & 1.040 & 1.100 & 1.099 & 1.114 & 1.078 & 1.086 & 2.7 \\
\hline 1,2-Dibromo-3-Chloropropane & 0.035 & 0.036 & 0.036 & 0.036 & 0.034 & 0.035 & 3.0 \\
\hline $1,2,4$-Trichlorobenzene & 0.674 & 0.720 & 0.684 & 0.712 & 0.684 & 0.695 & 2.8 \\
\hline 1,2,3-Trichlorobenzene & 0.429 & 0.455 & 0.432 & 0.456 & 0.426 & 0.440 & 3.4 \\
\hline
\end{tabular}


$6 \mathrm{C}-$ FORM VI VOA-3

VOLATILE ORGANICS INITIAL CALIBRATION DATA

Lab Name: TESTAMERICA BURLINGTON

Contract: $8 \mathrm{E}-00302$

Lab Code: STLV Case No.: CENTRA Mod. Ref No.: SDG No.: 200-1629

Instrument ID: J.i Calibration Date(s): 09/21/2010 09/21/2010

Heated Purge: ( $\mathrm{Y} / \mathrm{N}) \mathrm{N}$ Calibration Time(s) : 1435 1724

Purge Volume: 25.0 (mL)

GC Column: DB-624

ID: 0.20

(mm) Length: 25

(m)

\begin{tabular}{|c|c|c|c|c|c|c|c|}
\hline LAB EILE ID: & \multirow{2}{*}{\multicolumn{3}{|c|}{$\begin{array}{l}\text { RRF0.5 }=\frac{\text { JBM02.D }}{\text { RRE10 }}=\text { JBM05.D }\end{array}$}} & \multicolumn{4}{|c|}{ RRE1.0 = JBM03.D } \\
\hline RRE5.0 = JBMO 4.D & & & & RRE20 & $=\mathrm{JBMO}$ & 3. D & \\
\hline COMPOUND & RRF0. 5 & RRF 1.0 & RRF 5. 0 & RRF10 & RRF20 & $\overline{\mathrm{RRF}}$ & $\%$ RSD \\
\hline Vinyl Chloride-d3 & 0.329 & 0.374 & 0.324 & 0.332 & 0.313 & 0.335 & 6.9 \\
\hline Chloroethane-d5 & 0.269 & 0.297 & 0.274 & 0.273 & 0.259 & 0.274 & 5.2 \\
\hline 1,1-Dichloroethene-d2 & 0.534 & 0.546 & 0.524 & 0.531 & 0.512 & 0.530 & 2.3 \\
\hline 2-Butanone-d5 & 0.017 & 0.022 & 0.020 & 0.020 & 0.020 & 0.020 & 7.8 \\
\hline Chloroform-d & 0.474 & 0.497 & 0.471 & 0.485 & 0.471 & 0.480 & 2.4 \\
\hline 1,2-Dichloroethane-d4 & 0.158 & 0.155 & 0.152 & 0.153 & 0.148 & 0.153 & 2.5 \\
\hline Benzene-d6 & 1.364 & 1.461 & 1.408 & 1.443 & 1.373 & 1.410 & 3.0 \\
\hline 1,2-Dichloropropane-d6 & 0.373 & 0.397 & 0.385 & 0.337 & 0.366 & 0.372 & 6.1 \\
\hline Toluene-d8 & 1.321 & 1.416 & 1.382 & 1.436 & 1.374 & 1.386 & 3.2 \\
\hline trans-1,3-Dichloropropene-d4 & 0.241 & 0.251 & 0.259 & 0.273 & 0.260 & 0.257 & 4.7 \\
\hline 2-Hexanone-d5 & 0.022 & 0.025 & 0.024 & 0.026 & 0.024 & 0.024 & 7.1 \\
\hline $1,1,2,2-$ Tetrachloroethane-d2 & 0.100 & 0.119 & 0.121 & 0.123 & 0.117 & 0.116 & 8.0 \\
\hline 1,2 -Dichlorobenzene-d 4 & 0.706 & 0.698 & 0.678 & 0.691 & 0.676 & 0.690 & 1.8 \\
\hline
\end{tabular}

Report 1,4-Dioxane-d8 for Low-Medium VOA analysis only 
$7 A$ - FORM VII VOA-1

VOLATILE CONTINUING CALIBRATION DATA

Lab Name: TESTAMERICA BURLINGTON

Lab Code: STLV Cas

Instrument ID: J.i

Lab File Id: JBMD02.D

EPA Sample No. (VSTD\#\#\#\#): VSTD005JF

Heated Purge: ( $Y / N) N$ GC Column: DB-624

Purge volume: 25.0 (mL)
Contract: $8 \mathrm{E}-00302$

SDG No.: 200-1629

Calibration Date: 09/23/2010 Time: 1526 Init. Calib. Date(s):09/21/2010 09/21/2010

Init. Calib. Time(s) : $1435 \quad 1724$ ID: $0.20(\mathrm{~mm})$ Length: 25 (m)

\begin{tabular}{|c|c|c|c|c|c|}
\hline COMPOUND & $\overline{\mathrm{RRF}}$ & RRE5 . 0 & $\begin{array}{l}\text { MIN } \\
\text { RRF }\end{array}$ & $\because \mathrm{D}$ & MAX $\% D$ \\
\hline Dichlorodifluoromethane & 0.462 & 0.479 & 0.010 & 3.7 & 40.0 \\
\hline Chloromethane & 0.373 & 0.374 & 0.010 & 0.3 & 40.0 \\
\hline Vinyl chloride & 0.377 & 0.390 & 0.010 & 3.5 & 30.0 \\
\hline Bromomethane & 0.210 & 0.224 & 0.100 & 6.8 & 30.0 \\
\hline Chloroethane & 0.198 & 0.218 & 0.010 & 10.3 & 40.0 \\
\hline Trichlorofluoromethane & 0.586 & 0.603 & 0.010 & 3.0 & 40.0 \\
\hline 1,1-Dichloroethene & 0.285 & 0.290 & 0.100 & 1.8 & 30.0 \\
\hline 1,1,2-Trichloro-1,2,2-trifluoroethane & 0.326 & 0.329 & 0.010 & 1.0 & 40.0 \\
\hline Acetone & 0.013 & 0.012 & 0.010 & -8.1 & 40.0 \\
\hline Carbon disulfide & 0.793 & 0.791 & 0.010 & -0.3 & 40.0 \\
\hline Methyl acetate & 0.037 & 0.037 & 0.010 & 1.0 & 40.0 \\
\hline Methylene Chloride & 0.213 & 0.220 & 0.010 & 3.3 & 40.0 \\
\hline trans-1,2-Dichloroethene & 0.303 & 0.301 & 0.010 & -0.5 & 40.0 \\
\hline Methyl tert-butyl ether & 0.325 & 0.317 & 0.010 & -2.3 & 40.0 \\
\hline 1,1-Dichloroethane & 0.496 & 0.512 & 0.200 & 3.2 & 30.0 \\
\hline cis-1,2-Dichloroethene & 0.288 & 0.296 & 0.010 & 2.5 & 40.0 \\
\hline 2-Butanone & 0.019 & 0.019 & 0.010 & -1.0 & 40.0 \\
\hline Bromochloromethane & 0.086 & 0.086 & 0.050 & 0.1 & 30.0 \\
\hline Chloroform & 0.479 & 0.481 & 0.200 & 0.4 & 30.0 \\
\hline 1,1,1-Trichloroethane & 0.671 & 0.686 & 0.100 & 2.3 & 30.0 \\
\hline Cyclohexane & 0.661 & 0.679 & 0.010 & 2.7 & 40.0 \\
\hline Carbon tetrachloride & 0.610 & 0.621 & 0.100 & 1.9 & 30.0 \\
\hline Benzene & 1.555 & 1.585 & 0.400 & 2.0 & 30.0 \\
\hline 1,2-Dichloroethane & 0.194 & 0.195 & 0.100 & 0.4 & 30.0 \\
\hline Trichloroethene & 0.419 & 0.427 & 0.300 & 2.0 & 30.0 \\
\hline Methylcyclohexane & 0.532 & 0.545 & 0.010 & 2.6 & 40.0 \\
\hline
\end{tabular}

Report 1,4-Dioxane for Low/Medium VOA analysis only 
Lab Name: TESTAMERICA BURLINGTON

Lab Code: STLV Cas

Instrument ID: J.i Case No.: CENTRA Mod. Ref No.:

Contract: $8 \mathrm{E}-00302$

Lab File Id: JBMD02.D

EPA Sample No. (VSTD\#\#\#): VSTD005JF

Heated Purge: ( $Y / N)$ N GC Column: $D B-624$

$(\mathrm{mL})$
Calibration Date: 09/23/2010 Time: 1526

SDG NO.: 200-1629 Init. Calib. Date(s):09/21/2010 09/21/2010

Init. Calib. Time(s): $1435 \quad 1724$ ID: $0.20(\mathrm{~mm})$ Length: 25 (m)
Purge Volume: 25.0

\begin{tabular}{|c|c|c|c|c|c|}
\hline COMPOUND & $\overline{\mathrm{RRF}}$ & RRE5.0 & $\begin{array}{l}\text { MIN } \\
\text { RRF }\end{array}$ & $\frac{\circ}{0} \mathrm{D}$ & $\mathrm{MAX} \div \mathrm{D}$ \\
\hline 1,2-Dichloropropane & 0.286 & 0.301 & 0.010 & 5.3 & 40.0 \\
\hline Bromodichloromethane & 0.343 & 0.353 & 0.200 & 2.9 & 30.0 \\
\hline cis-1,3-Dichloropropene & 0.414 & 0.414 & 0.200 & -0.1 & 30.0 \\
\hline 4-Methyl-2-pentanone & 0.062 & 0.062 & 0.010 & -1.1 & 40.0 \\
\hline Toluene & 1.663 & 1.693 & 0.400 & 1.8 & 30.0 \\
\hline trans-1,3-Dichloropropene & 0.288 & 0.293 & 0.100 & 1.4 & 30.0 \\
\hline 1,1,2-Trichloroethane & 0.138 & 0.140 & 0.100 & 1.5 & 30.0 \\
\hline Tetrachloroethene & 0.375 & 0.379 & 0.100 & 1.0 & 30.0 \\
\hline 2-Hexanone & 0.045 & 0.040 & 0.010 & -11.2 & 40.0 \\
\hline Dibromochloromethane & 0.177 & 0.185 & 0.100 & 4.6 & 30.0 \\
\hline 1,2-Dibromoethane & 0.128 & 0.127 & 0.010 & -0.4 & 40.0 \\
\hline Chlorobenzene & 0.986 & 1.001 & 0.500 & 1.6 & 30.0 \\
\hline Ethylbenzene & 1.942 & 1.974 & 0.100 & 1.7 & 30.0 \\
\hline o-xylene & 0.667 & 0.686 & 0.300 & 2.8 & 30.0 \\
\hline $\mathrm{m}, \mathrm{p}$-xylene & 0.746 & 0.767 & 0.300 & 2.8 & 30.0 \\
\hline Styrene & 0.950 & 1.000 & 0.300 & 5.3 & 30.0 \\
\hline Bromoform & 0.172 & 0.174 & 0.050 & 1.3 & 30.0 \\
\hline Isopropylbenzene & 1.974 & 2.020 & 0.010 & 2.3 & 40.0 \\
\hline $1,1,2,2$-Tetrachloroethane & 0.122 & 0.121 & 0.100 & -1.3 & 30.0 \\
\hline 1,3 -Dichlorobenzene & 1.500 & 1.547 & 0.400 & 3.1 & 30.0 \\
\hline 1,4-Dichlorobenzene & 1.476 & 1.475 & 0.400 & -0.1 & 30.0 \\
\hline 1,2-Dichlorobenzene & 1.086 & 1.141 & 0.400 & 5.0 & 30.0 \\
\hline 1,2-Dibromo-3-Chloropropane & 0.035 & 0.035 & 0.010 & -2.0 & 40.0 \\
\hline $1,2,4$-Trichlorobenzene & 0.695 & 0.701 & 0.200 & 1.0 & 30.0 \\
\hline $1,2,3$-Trichlorobenzene & 0.440 & 0.452 & 0.200 & 2.8 & 30.0 \\
\hline
\end{tabular}


$7 C$ - FORM VII VOA-3

VOLATILE CONTINUING CALIBRATION DATA

Lab Name: TESTAMERICA BURLINGTON

Contract: $8 \mathrm{E}-00302$

Lab Code: STLV Case No.: CENTRA Mod. Ref No.: SDG No.: 200-1629

Instrument ID: J.i

Calibration Date: 09/23/2010 Time: 1526

Lab File Id: JBMD02.D

Init. Calib. Date(s):09/21/2010 09/21/2010

EPA Sample No. (VSTD\#\#\#\#) : VSTD005JF

Init. Calib. Time(s): $1435 \quad 1724$

Heated Purge: $(Y / N) N$ GC Column: $\mathrm{DB}-624$

ID: $0.20(\mathrm{~mm})$ Length: 25

(m)

Purge Volume: 25.0

(mL)

\begin{tabular}{|c|c|c|c|c|c|}
\hline COMPOUND & $\overline{\mathrm{RRF}}$ & RRF5. 0 & $\begin{array}{l}\text { MIN } \\
\text { RRE }\end{array}$ & $\div \mathrm{D}$ & $\operatorname{MAX} \div \mathrm{D}$ \\
\hline Vinyl Chloride-d3 & 0.335 & $0 . \overline{345}$ & 0.010 & 3.1 & 30.0 \\
\hline Chloroethane-d5 & 0.274 & 0.283 & 0.010 & 3.3 & 40.0 \\
\hline 1,1-Dichloroethene-d2 & 0.530 & 0.524 & 0.010 & -1.1 & 30.0 \\
\hline 2-Butanone-d5 & 0.020 & 0.019 & 0.010 & -2.4 & 40.0 \\
\hline Chloroform-d & 0.480 & 0.494 & 0.010 & 3.0 & 30.0 \\
\hline 1,2-Dichloroethane-d4 & 0.153. & 0.154 & 0.010 & 0.5 & 30.0 \\
\hline Benzene-d 6 & 1.410 & 1.446 & 0.010 & 2.6 & 30.0 \\
\hline 1,2-Dichloropropane-d6 & 0.372 & 0.390 & 0.010 & 4.9 & 40.0 \\
\hline Toluene-d8 & 1.386 & 1.401 & 0.010 & 1.1 & 30.0 \\
\hline trans-1,3-Dichloropropene-d4 & 0.257 & 0.255 & 0.010 & -0.6 & 30.0 \\
\hline $2-$ Hexanone-d5 & 0.024 & 0.024 & 0.010 & $-1 \cdot 3$ & 40.0 \\
\hline $1,1,2,2$-Tetrachloroethane-d2 & 0.116 & 0.121 & 0.010 & 4.4 & 30.0 \\
\hline 1,2 -Dichlorobenzene-d4 & 0.690 & 0.703 & 0.010 & 1.9 & 30.0 \\
\hline
\end{tabular}

Report 1,4-Dioxane-d8 for Low/Medium VOA analysis only 
$7 A$ - FORM VII VOA-1

VOLATILE CONTINUING CALIBRATION DATA

Lab Name: TESTAMERICA BURLINGTON

Lab Code: STLV

Instrument ID: J.i

Lab File Id: JBMD25.D

EPA Sample No. (VSTD\#\#\#): VSTD005FJ

Heated Purge: ( $Y / N) N$

Purge Volume: 25.0 GC Column: DB-624

(mI)
Contract: $8 \mathrm{E}-00302$

SDG NO.: 200-1629

Calibration Date: 09/24/2010 Time: 0218 Init. Calib. Date(s):09/21/2010 09/21/2010

Init. Calib. Time(s): $1435 \quad 1724$ ID: $0.20(\mathrm{~mm})$ Length: 25 (m)

\begin{tabular}{|c|c|c|c|c|c|}
\hline COMPOUND & $\overline{\mathrm{RRF}}$ & RRF5. 0 & $\begin{array}{l}\text { MIN } \\
\text { RRF }\end{array}$ & $\frac{\circ}{\partial} \mathrm{D}$ & $\operatorname{MAX} \div \mathrm{D}$ \\
\hline Dichlorodifluoromethane & 0.462 & 0.455 & 0.010 & -1.6 & 50.0 \\
\hline Chloromethane & 0.373 & 0.370 & 0.010 & -0.7 & 50.0 \\
\hline Vinyl chloride & 0.377 & 0.379 & 0.010 & 0.5 & 50.0 \\
\hline Bromomethane & 0.210 & 0.206 & 0.010 & -1.8 & 50.0 \\
\hline Chloroethane & 0.198 & 0.217 & 0.010 & 10.1 & 50.0 \\
\hline Trichlorofluoromethane & 0.586 & 0.601 & 0.010 & 2.5 & 50.0 \\
\hline 1,1-Dichloroethene & 0.285 & 0.293 & 0.010 & 2.9 & 50.0 \\
\hline 1,1,2-Trichloro-1,2,2-trifluoroethane & 0.326 & 0.317 & 0.010 & -2.7 & 50.0 \\
\hline Acetone & 0.013 & 0.012 & 0.010 & -7.0 & 50.0 \\
\hline Carbon disulfide & 0.793 & 0.783 & 0.010 & -1.3 & 50.0 \\
\hline Methyl acetate & 0.037 & 0.031 & 0.010 & -15.5 & 50.0 \\
\hline Methylene Chloride & 0.213 & 0.217 & 0.010 & 2.1 & 50.0 \\
\hline trans-1,2-Dichloroethene & 0.303 & 0.310 & 0.010 & 2.4 & 50.0 \\
\hline Methyl tert-butyl ether & 0.325 & 0.336 & 0.010 & 3.3 & 50.0 \\
\hline 1,1-Dichloroethane & 0.496 & 0.517 & 0.010 & 4.3 & 50.0 \\
\hline cis-1,2-Dichloroethene & 0.288 & 0.293 & 0.010 & 1.6 & 50.0 \\
\hline 2-Butanone & 0.019 & 0.020 & 0.010 & 4.8 & 50.0 \\
\hline Bromochloromethane & 0.086 & 0.087 & 0.010 & 1.2 & 50.0 \\
\hline Chloroform & 0.479 & 0.489 & 0.010 & 2.0 & 50.0 \\
\hline 1,1,1-Trichloroethane & 0.671 & 0.671 & 0.010 & 0.0 & 50.0 \\
\hline Cyclohexane & 0.661 & 0.671 & 0.010 & 1.5 & 50.0 \\
\hline Carbon tetrachloride & 0.610 & 0.618 & 0.010 & 1.3 & 50.0 \\
\hline Benzene & 1.555 & 1.600 & 0.010 & 2.9 & 50.0 \\
\hline 1,2-Dichloroethane & 0.194 & 0.199 & 0.010 & 2.5 & 50.0 \\
\hline Trichloroethene & 0.419 & 0.425 & 0.010 & 1.4 & 50.0 \\
\hline Methylcyclohexane & 0.532 & 0.529 & 0.010 & -0.5 & 50.0 \\
\hline
\end{tabular}

Report 1,4-Dioxane for Low/Medium VOA analysis only 
Lab Name: TESTAMERICA BURLINGTON

Lab Code: STLV Case No.: CENTRA Mod. Ref No.:

Contract: $8 \mathrm{E}-00302$

Instrument ID: J.i

Lab File Id: JBMD25.D

Calibration Date: 09/24/2010 Time: 0218

EPA Sample No.(VSTD\#\#\#\#): VSTD005FJ Init. Calib. Date(s):09/21/2010 09/21/2010

Heated Purge: ( $Y / N)$

Purge Volume: 25.0 GC Column: $\mathrm{DB}-624$

Init. Calib. Time(s): 1435 1724 (mL) (m)

\begin{tabular}{|c|c|c|c|c|c|}
\hline COMPOUND & $\overline{\mathrm{RRF}}$ & $\operatorname{RRF} 5.0$ & $\begin{array}{l}\text { MIN } \\
\text { RRF }\end{array}$ & $\% \mathrm{D}$ & $\operatorname{MAX} \div D$ \\
\hline 1,2-Dichloropropane & 0.286 & 0.300 & 0.010 & $\overline{4.9}$ & 50.0 \\
\hline Bromodichloromethane & 0.343 & 0.362 & 0.010 & 5.6 & 50.0 \\
\hline cis-1,3-Dichloropropene & 0.414 & 0.417 & 0.010 & 0.6 & 50.0 \\
\hline 4-Methyl-2-pentanone & 0.062 & 0.065 & 0.010 & 3.9 & 50.0 \\
\hline Toluene & 1.663 & 1.683 & 0.010 & 1.2 & 50.0 \\
\hline trans-1,3-Dichloropropene & 0.288 & 0.300 & 0.010 & 4.1 & 50.0 \\
\hline $1,1,2$-Trichloroethane & 0.138 & 0.143 & 0.010 & 3.9 & 50.0 \\
\hline Tetrachloroethene & 0.375 & 0.374 & 0.010 & -0.4 & 50.0 \\
\hline 2-Hexanone & 0.045 & 0.041 & 0.010 & -9.1 & 50.0 \\
\hline Dibromochloromethane & 0.177 & 0.196 & 0.010 & 11.2 & 50.0 \\
\hline 1,2-Dibromoethane & 0.128 & 0.131 & 0.010 & 2.6 & 50.0 \\
\hline Chlorobenzene & 0.986 & 0.987 & 0.010 & 0.1 & 50.0 \\
\hline Ethylbenzene & 1.942 & 1.959 & 0.010 & 0.9 & 50.0 \\
\hline o-xylene & 0.667 & 0.677 & 0.010 & 1.5 & 50.0 \\
\hline$m, p-x y$ lene & 0.746 & 0.766 & 0.010 & 2.7 & 50.0 \\
\hline Styrene & 0.950 & 0.978 & 0.010 & 3.0 & 50.0 \\
\hline Bromoform & 0.172 & 0.191 & 0.010 & 10.8 & 50.0 \\
\hline Isopropylbenzene & 1.974 & 1.960 & 0.010 & -0.7 & 50.0 \\
\hline $1,1,2,2$-Tetrachloroethane & 0.122 & 0.129 & 0.010 & 5.3 & 50.0 \\
\hline 1,3-Dichlorobenzene & 1.500 & 1.510 & 0.010 & 0.7 & 50.0 \\
\hline 1,4-Dichlorobenzene & 1.476 & 1.464 & 0.010 & -0.8 & 50.0 \\
\hline 1,2-Dichlorobenzene & 1.086 & 1.127 & 0.010 & 3.7 & 50.0 \\
\hline 1,2-Dibromo-3-Chloropropane & 0.035 & 0.037 & 0.010 & 3.9 & 50.0 \\
\hline 1,2,4-Trichlorobenzene & 0.695 & 0.646 & 0.010 & -7.0 & 50.0 \\
\hline 1,2,3-Trichlorobenzene & 0.440 & 0.408 & 0.010 & -7.1 & 50.0 \\
\hline
\end{tabular}


Lab Name: TESTAMERICA BURLINGTON

Lab Code: STLV Case No.: CENTRA Mod. Ref No.:

Contract: $8 \mathrm{E}-00302$

Instrument ID: J.i

Calibration Date: 09/24/2010 Time: 0218

Lab File Id: JBMD25.D Init. Calib. Date(s):09/21/2010 09/21/2010

EPA Sample No. (VSTD\#\#\#\#): VSTD005FJ

Init. Calib. Time(s): $1435 \quad 1724$

Heated Purge: ( $\mathrm{Y} / \mathrm{N}) \mathrm{N}$ GC Column: DB-624

$(\mathrm{mL})$

Purge volume: 25.0 ID: $\underline{0.20}(\mathrm{~mm})$ Length: 25

(m)

\begin{tabular}{|c|c|c|c|c|c|}
\hline COMPOUND & $\overline{\mathrm{RRF}}$ & RRF 5. 0 & $\begin{array}{l}\text { MIN } \\
\text { RRF }\end{array}$ & $\because \mathrm{D}$ & $\operatorname{MAX} \div \mathrm{D}$ \\
\hline Vinyl Chloride-d3 & 0.335 & 0.340 & 0.010 & 1.7 & 50.0 \\
\hline Chloroethane-d5 & 0.274 & 0.276 & 0.010 & 0.7 & 50.0 \\
\hline 1,1-Dichloroethene-d2 & 0.530 & 0.532 & 0.010 & 0.4 & 50.0 \\
\hline 2-Butanone-d5 & 0.020 & 0.021 & 0.010 & 8.2 & 50.0 \\
\hline Chloroform-d & 0.480 & 0.508 & 0.010 & 5.9 & 50.0 \\
\hline 1,2-Dichloroethane-d4 & 0.153 & 0.158 & 0.010 & 3.3 & 50.0 \\
\hline Benzene-d6 & 1.410 & 1.453 & 0.010 & 3.1 & 50.0 \\
\hline 1,2-Dichloropropane-d6 & 0.372 & 0.395 & 0.010 & 6.1 & 50.0 \\
\hline Toluene-d8 & 1.386 & 1.406 & 0.010 & 1.4 & 50.0 \\
\hline trans-1,3-Dichloropropene-d4 & 0.257 & 0.260 & 0.010 & 1.2 & 50.0 \\
\hline 2-Hexanone-d5 & 0.024 & 0.025 & 0.010 & 4.5 & 50.0 \\
\hline $1,1,2,2-$ Tetrachloroethane-d2 & 0.116 & 0.124 & 0.010 & 7.2 & 50.0 \\
\hline 1,2-Dichlorobenzene-d4 & 0.690 & 0.716 & 0.010 & 3.8 & 50.0 \\
\hline
\end{tabular}

Report 1,4-Dioxane-d8 for Low/Medium VOA analysis only . 
Lab Name: TESTAMERICA BURLINGTON

Lab code: STLV

Instrument ID: J.i

Lab File Id: JBME02.D

EPA Sample No. (VSTD\#\#\#\#): VSTD005JG

Heated Purge: ( $\mathrm{Y} / \mathrm{N}) \mathrm{N}$

Purge volume: 25.0
Contract: $8 E-00302$

SDG No.: 200-1629

Calibration Date: 09/24/2010 Time: 0641 Init. Calib. Date(s):09/21/2010 09/21/2010

Init. Calib. Time(s): $1435 \quad 1724$ GC Column: DB-624 ID: $0.20(\mathrm{~mm})$ Length: 25 (m) $(m L)$

\begin{tabular}{|c|c|c|c|c|c|}
\hline COMPOUND & $\overline{\mathrm{RRE}}$ & RRE5. 0 & $\begin{array}{l}\text { MIN } \\
\text { RRE }\end{array}$ & $\because \mathrm{D}$ & MAX $\because D$ \\
\hline Dichlorodifluoromethane & 0.462 & 0.513 & 0.010 & 11.0 & 40.0 \\
\hline Chloromethane & 0.373 & 0.382 & 0.010 & 2.4 & 40.0 \\
\hline Vinyl chloride & 0.377 & 0.420 & 0.010 & 11.4 & 30.0 \\
\hline Bromomethane & 0.210 & 0.245 & 0.100 & 16.9 & 30.0 \\
\hline Chloroethane & 0.198 & 0.240 & 0.010 & 21.5 & 40.0 \\
\hline Trichlorofluoromethane & 0.586 & 0.636 & 0.010 & 8.5 & 40.0 \\
\hline 1,1-Dichloroethene & 0.285 & 0.304 & 0.100 & 6.8 & 30.0 \\
\hline 1,1,2-Trichloro-1,2,2-trifluoroethane & 0.326 & 0.351 & 0.010 & 7.7 & 40.0 \\
\hline Acetone & 0.013 & 0.012 & 0.010 & -6.8 & 40.0 \\
\hline Carbon disulfide & 0.793 & 0.859 & 0.010 & 8.2 & 40.0 \\
\hline Methyl acetate & 0.037 & 0.037 & 0.010 & 1.1 & 40.0 \\
\hline Methylene Chloride & 0.213 & 0.232 & 0.010 & 8.9 & 40.0 \\
\hline trans-1,2-Dichloroethene & 0.303 & 0.329 & 0.010 & 8.7 & 40.0 \\
\hline Methyl tert-butyl ether & 0.325 & 0.316 & 0.010 & -2.9 & 40.0 \\
\hline 1,1-Dichloroethane & 0.496 & 0.535 & 0.200 & 8.0 & 30.0 \\
\hline cis-1,2-Dichloroethene & 0.288 & 0.311 & 0.010 & 7.8 & 40.0 \\
\hline 2-Butanone & 0.019 & 0.019 & 0.010 & -2.6 & 40.0 \\
\hline Bromochloromethane & 0.086 & 0.092 & 0.050 & 6.4 & 30.0 \\
\hline Chloroform & 0.479 & 0.504 & 0.200 & 5.3 & 30.0 \\
\hline $1,1,1$-Trichloroethane & 0.671 & 0.711 & 0.100 & 6.1 & 30.0 \\
\hline Cyclohexane & 0.661 & 0.716 & 0.010 & 8.3 & 40.0 \\
\hline Carbon tetrachloride & 0.610 & 0.655 & 0.100 & 7.3 & 30.0 \\
\hline Benzene & 1.555 & 1.658 & 0.400 & 6.6 & 30.0 \\
\hline 1,2-Dichloroethane & 0.194 & 0.201 & 0.100 & 3.3 & 30.0 \\
\hline Trichloroethene & 0.419 & 0.437 & 0.300 & 4.4 & 30.0 \\
\hline Methylcyclohexane & 0.532 & 0.577 & 0.010 & 8.5 & 40.0 \\
\hline
\end{tabular}

Report 1,4-Dioxane for Low/Medium VOA analysis only 
Lab Name: TESTAMERICA BURIINGTON

Lab Code: STLV

Instrument ID: J.i

Lab File Id: JBME02.D

EPA Sample No. (VSTD\#\#\#\#) : VSTD005JG

Heated Purge: ( $\mathrm{Y} / \mathrm{N}$ ) $\mathrm{N}$

Purge Volume: 25.0
Contract: $8 \mathrm{E}-00302$

Case No.: CENTRA Mod. Ref No.:
SDG No.: 200-1629 Init. Calib. Date(s):09/21/2010 09/21/2010

Init. Calib. Time(s): $1435 \quad 1724$

(m)

\begin{tabular}{|c|c|c|c|c|c|}
\hline COMPOUND & $\overline{\mathrm{RRF}}$ & RRE5.0 & $\begin{array}{l}\text { MIN } \\
\text { RRE }\end{array}$ & $\because \mathrm{D}$ & $\operatorname{MAX} \div \mathrm{D}$ \\
\hline 1,2-Dichloropropane & 0.286 & 0.315 & 0.010 & 10.2 & 40.0 \\
\hline Bromodichloromethane & 0.343 & 0.374 & 0.200 & 9.0 & 30.0 \\
\hline cis-1,3-Dichloropropene & 0.414 & 0.438 & 0.200 & 5.8 & 30.0 \\
\hline 4-Methyl-2-pentanone & 0.062 & 0.061 & 0.010 & -1.5 & 40.0 \\
\hline Toluene & 1.663 & 1.785 & 0.400 & 7.3 & 30.0 \\
\hline trans-1,3-Dichloropropene & 0.288 & 0.304 & 0.100 & 5.4 & 30.0 \\
\hline $1,1,2$-Trichloroethane & 0.138 & 0.142 & 0.100 & 3.1 & 30.0 \\
\hline Tetrachloroethene & 0.375 & 0.403 & 0.100 & 7.5 & 30.0 \\
\hline 2-Hexanone & 0.045 & 0.039 & 0.010 & -13.6 & 40.0 \\
\hline Dibromochloromethane & 0.177 & 0.189 & 0.100 & 6.9 & 30.0 \\
\hline 1,2-Dibromoethane & 0.128 & 0.127 & 0.010 & -0.4 & 40.0 \\
\hline Chlorobenzene & 0.986 & 1.026 & 0.500 & 4.1 & 30.0 \\
\hline Ethylbenzene & 1.942 & 2.100 & 0.100 & 8.1 & 30.0 \\
\hline o-xylene & 0.667 & 0.718 & 0.300 & 7.6 & 30.0 \\
\hline m,p-xylene & 0.746 & 0.805 & 0.300 & 8.0 & 30.0 \\
\hline Styrene & 0.950 & 1.020 & 0.300 & 7.4 & 30.0 \\
\hline Bromoform & 0.172 & 0.186 & 0.050 & 8.1 & 30.0 \\
\hline Isopropylbenzene & 1.974 & 2.129 & 0.010 & 7.8 & 40.0 \\
\hline $1,1,2,2$-Tetrachloroethane & 0.122 & 0.123 & 0.100 & 0.5 & 30.0 \\
\hline 1,3-Dichlorobenzene & 1.500 & 1.592 & 0.400 & 6.1 & 30.0 \\
\hline 1,4-Dichlorobenzene & 1.476 & 1.533 & 0.400 & 3.9 & 30.0 \\
\hline 1,2-Dichlorobenzene & 1.086 & 1.170 & 0.400 & 7.7 & 30.0 \\
\hline 1,2-Dibromo-3-Chloropropane & 0.035 & 0.034 & 0.010 & -2.8 & 40.0 \\
\hline $1,2,4-T r i c h l o r o b e n z e n e$ & 0.695 & 0.711 & 0.200 & 2.3 & 30.0 \\
\hline 1,2,3-Trichlorobenzene & 0.440 & 0.454 & 0.200 & 3.3 & 30.0 \\
\hline
\end{tabular}

(mi)

ID: $0.20(\mathrm{~mm})$ Iength: 25 
$7 C$ - FORM VII VOA-3

VOLATILE CONTINUING CALIBRATION DATA

Lab Name: TESTAMERICA BURLINGTON

Contract: $8 \mathrm{E}-00302$

Lab Code: STLV Case No.: CENTRA Mod. Ref No.:

SDG No.: 200-1629

Instrument ID: J.i

Calibration Date: 09/24/2010 Time: 0641

Lab File Id: JBME02.D Init. Calib. Date(s):09/21/2010 09/21/2010

EPA Sample No. (VSTD\#\#\#): VSTD005JG

Init. Calib. Time(s): $1435 \quad 1724$

Heated Purge: ( $Y / N) \mathrm{N}$ GC Column: DB-624 ID: $0.20(\mathrm{~mm})$ Length: 25 (m)

Purge Volume: 25.0

$(\mathrm{mL})$

\begin{tabular}{|c|c|c|c|c|c|}
\hline COMPOUND & $\overline{\mathrm{RRF}}$ & RRE5.0 & $\begin{array}{l}\text { MIN } \\
\text { RRF }\end{array}$ & $\because \mathrm{D}$ & $\operatorname{MAX} \div \mathrm{D}$ \\
\hline Vinyl Chloride-d3 & 0.335 & 0.372 & 0.010 & 11.1 & 30.0 \\
\hline Chloroethane-d5 & 0.274 & 0.296 & 0.010 & 8.1 & 40.0 \\
\hline 1,1-Dichloroethene-d2 & 0.530 & 0.564 & 0.010 & 6.5 & 30.0 \\
\hline 2-Butanone-d5 & 0.020 & 0.019 & 0.010 & -1.8 & 40.0 \\
\hline Chloroform-d & 0.480 & 0.524 & 0.010 & 9.1 & 30.0 \\
\hline 1,2-Dichloroethane-d4 & 0.153 & 0.161 & 0.010 & 4.7 & 30.0 \\
\hline Benzene-d6 & 1.410 & 1.512 & 0.010 & 7.2 & 30.0 \\
\hline 1,2-Dichloropropane-d6 & 0.372 & 0.353 & 0.010 & -5.0 & 40.0 \\
\hline Toluene-d8 & 1.386 & 1.473 & 0.010 & 6.3 & 30.0 \\
\hline trans-1,3-Dichloropropene-d4 & 0.257 & 0.271 & 0.010 & 5.5 & 30.0 \\
\hline 2-Hexanone-d5 & 0.024 & 0.023 & 0.010 & -4.1 & 40.0 \\
\hline $1,1,2,2-$ Tetrachloroethane-d2 & 0.116 & 0.119 & 0.010 & 2.5 & 30.0 \\
\hline 1,2-Dichlorobenzene-d4 & 0.690 & 0.728 & 0.010 & 5.5 & 30.0 \\
\hline
\end{tabular}

Report 1,4-Dioxane-d8 for Low/Medium VOA analysis only 
Lab Name: TESTAMERICA BURLINGTON

Lab Code: STLV

Instrument ID: J.i

Lab File Id: JBME17. D

EPA Sample No.(VSTD\#\#\#\#) : VSTD005GJ

Heated Purge: ( $Y / N) N$

Purge Volume: 25.0
Contract: $8 \mathrm{E}-00302$

SDG No.: 200-1629

Calibration Date: 09/24/2010 Time: 1354 Init. Calib. Date(s):09/21/2010 09/21/2010

Init. Calib. Time(s): $1435 \quad 1724$ GC Column: DB-624 ID: $0.20(\mathrm{~mm})$ Length: 25 (m) (mI)

\begin{tabular}{|c|c|c|c|c|c|}
\hline COMPOUND & $\overline{\mathrm{RRF}}$ & RRF5.0 & $\begin{array}{l}\text { MIN } \\
\text { RRF }\end{array}$ & $\because D$ & $\operatorname{MAX} \div \mathrm{D}$ \\
\hline Dichlorodifluoromethane & 0.462 & 0.456 & 0.010 & -1.3 & 50.0 \\
\hline Chloromethane & 0.373 & 0.376 & 0.010 & 0.9 & 50.0 \\
\hline Vinyl chloride & 0.377 & 0.374 & 0.010 & -0.9 & 50.0 \\
\hline Bromomethane & 0.210 & 0.209 & 0.010 & -0.6 & 50.0 \\
\hline Chloroethane & 0.198 & 0.215 & 0.010 & 9.0 & 50.0 \\
\hline Trichlorofluoromethane & 0.586 & 0.607 & 0.010 & 3.7 & 50.0 \\
\hline 1,1-Dichloroethene & 0.285 & 0.286 & 0.010 & 0.4 & 50.0 \\
\hline 1,1,2-Trichloro-1,2,2-trifluoroethane & 0.326 & 0.328 & 0.010 & 0.6 & 50.0 \\
\hline Acetone & 0.013 & 0.012 & 0.010 & -6.6 & 50.0 \\
\hline Carbon disulfide & 0.793 & 0.777 & 0.010 & -2.1 & 50.0 \\
\hline Methyl acetate & 0.037 & 0.036 & 0.010 & -2.2 & 50.0 \\
\hline Methylene Chloride & 0.213 & 0.220 & 0.010 & 3.5 & 50.0 \\
\hline trans-1,2-Dichloroethene & 0.303 & 0.314 & 0.010 & 3.8 & 50.0 \\
\hline Methyl tert-butyl ether & 0.325 & 0.339 & 0.010 & 4.2 & 50.0 \\
\hline 1,1-Dichloroethane & 0.496 & 0.520 & 0.010 & 4.8 & 50.0 \\
\hline cis-1,2-Dichloroethene & 0.288 & 0.300 & 0.010 & 4.0 & 50.0 \\
\hline 2-Butanone & 0.019 & 0.019 & 0.010 & 0.1 & 50.0 \\
\hline Bromochloromethane & 0.086 & 0.090 & 0.010 & 4.5 & 50.0 \\
\hline Chloroform & 0.479 & 0.498 & 0.010 & 3.8 & 50.0 \\
\hline 1,1,1-Trichloroethane & 0.671 & 0.668 & 0.010 & -0.4 & 50.0 \\
\hline Cyclohexane & 0.661 & 0.664 & 0.010 & 0.5 & 50.0 \\
\hline Carbon tetrachloride & 0.610 & 0.611 & 0.010 & 0.1 & 50.0 \\
\hline Benzene & 1.555 & 1.575 & 0.010 & 1.3 & 50.0 \\
\hline 1,2-Dichloroethane & 0.194 & 0.197 & 0.010 & 1.4 & 50.0 \\
\hline Trichloroethene & 0.419 & 0.427 & 0.010 & 1.8 & 50.0 \\
\hline Methylcyclohexane & 0.532 & 0.533 & 0.010 & 0.3 & 50.0 \\
\hline
\end{tabular}

Report 1,4-Dioxane for Low/Medium VOA analysis only 
$7 \mathrm{~B}$ - FORM VII VOA-2

VOLATILE CONTINUING CALIBRATION DATA

Lab Name: TESTAMERICA BURLINGTON Lab Code: STLV Case No.: CENTRA Mod. Ref No.:

Contract: $8 \mathrm{E}-00302$

Instrument ID: J.i

Lab File Id: JBME17.D

EPA Sample No.(VSTD\#\#\#\#) : VSTD005GJ

Heated Purge: ( $Y / N)$ N GC Column: DB-624

Purge Volume: 25.0 (mL)
Calibration Date: 09/24/2010 Time: 1354 Init. Calib. Date(s):09/21/2010 09/21/2010

Init. Calib. Time(s): $1435 \quad 1724$ ID: $0.20(\mathrm{~mm})$ Length: 25 (m)

\begin{tabular}{|c|c|c|c|c|c|}
\hline COMPOUND & $\overline{\mathrm{RRF}}$ & RRF5.0 & $\begin{array}{l}\text { MIN } \\
\text { RRF }\end{array}$ & $\because \mathrm{D}$ & $\operatorname{MAX} \div \mathrm{D}$ \\
\hline 1,2-Dichloropropane & 0.286 & 0.301 & 0.010 & 5.2 & 50.0 \\
\hline Bromodichloromethane & 0.343 & 0.357 & 0.010 & 4.0 & 50.0 \\
\hline cis-1,3-Dichloropropene & 0.414 & 0.432 & 0.010 & 4.2 & 50.0 \\
\hline 4-Methyl-2-pentanone & 0.062 & 0.064 & 0.010 & 2.9 & 50.0 \\
\hline Toluene & 1.663 & 1.670 & 0.010 & 0.4 & 50.0 \\
\hline trans-1,3-Dichloropropene & 0.288 & 0.306 & 0.010 & 6.2 & 50.0 \\
\hline 1,1,2-Trichloroethane & 0.138 & 0.140 & 0.010 & 2.1 & 50.0 \\
\hline Tetrachloroethene & 0.375 & 0.368 & 0.010 & -1.9 & 50.0 \\
\hline 2-Hexanone & 0.045 & 0.042 & 0.010 & -8.2 & 50.0 \\
\hline Dibromochloromethane & 0.177 & 0.185 & 0.010 & 4.8 & 50.0 \\
\hline 1,2-Dibromoethane & 0.128 & 0.132 & 0.010 & 3.4 & 50.0 \\
\hline Chlorobenzene & 0.986 & 0.993 & 0.010 & 0.7 & 50.0 \\
\hline Ethylbenzene & 1.942 & 1.951 & 0.010 & 0.5 & 50.0 \\
\hline o-Xylene & 0.667 & 0.685 & 0.010 & 2.7 & 50.0 \\
\hline$m, p$-xylene & 0.746 & 0.755 & 0.010 & 1.2 & 50.0 \\
\hline Styrene & 0.950 & 0.967 & 0.010 & 1.8 & 50.0 \\
\hline Bromoform & 0.172 & 0.187 & 0.010 & 8.6 & 50.0 \\
\hline Isopropylbenzene & 1.974 & 1.966 & 0.010 & -0.4 & 50.0 \\
\hline $1,1,2,2$-Tetrachloroethane & 0.122 & 0.127 & 0.010 & 4.1 & 50.0 \\
\hline 1,3-Dichlorobenzene & 1.500 & 1.515 & 0.010 & 1.0 & 50.0 \\
\hline 1,4-Dichlorobenzene & 1.476 & 1.491 & 0.010 & 1.0 & 50.0 \\
\hline 1,2-Dichlorobenzene & 1.086 & 1.137 & 0.010 & 4.6 & 50.0 \\
\hline 1,2-Dibromo-3-Chloropropane & 0.035 & 0.034 & 0.010 & -3.6 & 50.0 \\
\hline 1,2,4-Trichlorobenzene & 0.695 & 0.687 & 0.010 & -1.1 & 50.0 \\
\hline 1,2,3-Trichlorobenzene & 0.440 & 0.448 & 0.010 & 1.9 & 50.0 \\
\hline
\end{tabular}


$7 \mathrm{C}-$ FORM VII VOA-3

VOLATILE CONTINUING CALIBRATION DATA

Lab Name: TESTAMERICA BURLINGTON

Contract: $8 \mathrm{E}-00302$

Lab Code: STLV Case No.: CENTRA Mod. Ref No.: SDG No.: 200-1629

Instrument ID: J.i Calibration Date: 09/24/2010 Time: 1354

Lab File Id: JBME17. D Init. Calib. Date(s):09/21/2010 09/21/2010

EPA Sample No. (VSTD\#\#\#\#) : VSTD005GJ

Init. Calib. Time(s): $1435 \quad 1724$

Heated Purge: $(\mathrm{Y} / \mathrm{N}) \mathrm{N}$ GC Column: DB-624 ID: $0.20(\mathrm{~mm})$ Length: 25 (m)

Purge Volume: 25.0 (mI)

\begin{tabular}{|c|c|c|c|c|c|}
\hline COMPOUND & $\overline{\mathrm{RRF}}$ & $\operatorname{RRF} 5.0$ & $\begin{array}{l}\text { MIN } \\
\text { RRF } \\
\end{array}$ & $\div \mathrm{D}$ & MAX $\cong \mathrm{D}$ \\
\hline Vinyl Chloride-d3 & 0.335 & 0.339 & 0.010 & 1.4 & 50.0 \\
\hline Chloroethane-d5 & 0.274 & 0.280 & 0.010 & 2.1 & 50.0 \\
\hline 1,1-Dichloroethene-d2 & 0.530 & 0.534 & 0.010 & 0.8 & 50.0 \\
\hline 2-Butanone-d5 & 0.020 & 0.021 & 0.010 & 7.4 & 50.0 \\
\hline Chloroform-d & 0.480 & 0.504 & 0.010 & 5.0 & 50.0 \\
\hline 1,2-Dichloroethane-d4 & 0.153 & 0.160 & 0.010 & 4.3 & 50.0 \\
\hline Benzene-d6 & 1.410 & 1.437 & 0.010 & 1.9 & 50.0 \\
\hline 1,2-Dichloropropane-d6 & 0.372 & 0.389 & 0.010 & 4.6 & 50.0 \\
\hline Toluene-d8 & 1.386 & 1.384 & 0.010 & -0.1 & 50.0 \\
\hline trans-1,3-Dichloropropene-d4 & 0.257 & 0.261 & 0.010 & 1.5 & 50.0 \\
\hline 2-Hexanone-d5 & 0.024 & 0.025 & 0.010 & 4.5 & 50.0 \\
\hline $1,1,2,2$-Tetrachloroethane-d2 & 0.116 & 0.123 & 0.010 & 6.2 & 50.0 \\
\hline 1,2-Dichlorobenzene-d4 & 0.690 & 0.715 & 0.010 & 3.7 & 50.0 \\
\hline
\end{tabular}

Report 1,4-Dioxane-d8 for Low/Medium VOA analysis only 
Lab Name: TESTAMERICA BURIINGTON

Lab Code: STLV

Matrix: (SOIL/SED/WATER)

Sample wt/vol: 25.0

Case No.: CENTRA Mod. Ref No.:

Contract: $8 \mathrm{E}-00302$

Level: (TRACE/IOW/MED) TRACE

\% Moisture: not dec.

GC Column: DB-624

ID: 0.20

Water

$(\mathrm{g} / \mathrm{mL}) \mathrm{mL}$

Soil Extract Volume:

Purge Volume: 25.0 mm)

(uL)

(mL)
Lab File ID: JBMD04.D

Date Received:

Dilution Factor: 1.0

Soil Aliquot volume:
SDG NO.: 200-1629

Lab Sample ID: MB 200-7052/4

Date Analyzed: 09/23/2010 (uL)

\begin{tabular}{|c|c|c|c|}
\hline CAS NO. & COMPOUND & $\begin{array}{l}\text { CONCENTRATION UNITS: } \\
(\mathrm{ug} / \mathrm{L} \text { or } \mathrm{ug} / \mathrm{kg}) \mathrm{ug} / \mathrm{L}\end{array}$ & $Q$ \\
\hline $75-71-8$ & Dichlorodifluoromethane & 0.50 & $\overline{\mathrm{U}}$ \\
\hline $74-87-3$ & Chloromethane & 0.50 & $\mathrm{U}$ \\
\hline $75-01-4$ & Vinyl chloride & 0.50 & $\mathrm{U}$ \\
\hline $74-83-9$ & Bromomethane & 0.50 & $\overline{\mathrm{U}}$ \\
\hline $75-00-3$ & Chloroethane & 0.50 & $\mathrm{U}$ \\
\hline $75-69-4$ & Trichlorofluoromethane & 0.50 & $\mathrm{U}$ \\
\hline $75-35-4$ & 1,1-Dichloroethene & 0.50 & $\overline{\mathrm{U}}$ \\
\hline $.76-13-1$ & 1,1,2-Trichloro-1,2,2-trifluoroethane & 0.50 & $\bar{U}$ \\
\hline $67-64-1$ & Acetone & 3.0 & $\mathrm{~J}$ \\
\hline $75-15-0$ & Carbon disulfide & 0.50 & $\mathrm{U}$ \\
\hline $79-20-9$ & Methyl acetate & 0.50 & $\mathrm{U}$ \\
\hline $75-09-2$ & Methylene Chloride & 0.50 & $\mathrm{U}$ \\
\hline $156-60-5$ & trans-1,2-Dichloroethene & 0.50 & $\mathrm{U}$ \\
\hline $1634-04-4$ & Methyl tert-butyl ether & 0.50 & $\mathrm{U}$ \\
\hline $75-34-3$ & 1,1-Dichloroethane & 0.50 & $\mathrm{U}$ \\
\hline $156-59-2$ & cis-1,2-Dichloroethene & 0.50 & $\mathrm{U}$ \\
\hline $78-93-3$ & 2-Butanone & 5.0 & $\mathrm{U}$ \\
\hline $74-97-5$ & Bromochloromethane & 0.50 & $\mathrm{U}$ \\
\hline $67-66-3$ & Chloroform & 0.50 & $\mathrm{U}$ \\
\hline $71-55-6$ & 1,1,1-Trichloroethane & 0.50 & $\mathrm{U}$ \\
\hline $110-82-7$ & Cyclohexane & 0.50 & $\mathrm{U}$ \\
\hline $56-23-5$ & Carbon tetrachloride & 0.50 & $\mathrm{U}$ \\
\hline $71-43-2$ & Benzene & 0.50 & $\mathrm{U}$ \\
\hline $107-06-2$ & 1,2-Dichloroethane & 0.50 & $\mathrm{U}$ \\
\hline
\end{tabular}

Report 1,4-Dioxane for Low-Medium VOA analysis only 
$1 B$ - FORM I VOA-2

VOLATILE ORGANICS ANALYSIS DATA SHEET
EPA SAMPLE NO.

VBLKJF
Lab Name: TESTAMERICA BURLINGTON

Lab Code: STLV

Matrix: (SOIL/SED/WATER)
Sample wt/vol: 25.0

\section{Water}

$(\mathrm{g} / \mathrm{mL}) \mathrm{mI}$

Level: (TRACE/LOW/MED) TRACE

\% Moisture: not dec.

GC Column: DB-624

Soil Extract Volume: ID: $0.20 \quad(\mathrm{~mm})$

Purge Volume: 25.0

(mL)
Contract: $8 \mathrm{E}-00302$

SDG No.: 200-1629

\begin{tabular}{|c|c|c|c|}
\hline CAS NO. & COMPOUND & $\begin{array}{l}\text { CONCENTRATION UNITS: } \\
(\mathrm{ug} / \mathrm{L} \text { or } \mathrm{ug} / \mathrm{kg}) \mathrm{ug} / \mathrm{L}\end{array}$ & $Q$ \\
\hline $79-01-6$ & Trichloroethene & 0.50 & $\overline{\mathrm{U}}$ \\
\hline $108-87-2$ & Methylcyclohexane & 0.50 & $\mathrm{U}$ \\
\hline $78-87-5$ & 1,2-Dichloropropane & 0.50 & $\mathrm{U}$ \\
\hline $75-27-4$ & Bromodichloromethane & 0.50 & $\mathrm{U}$ \\
\hline $10061-01-5$ & cis-1,3-Dichloropropene & 0.50 & $\mathrm{U}$ \\
\hline $108-10-1$ & 4-Methyl-2-pentanone & 5.0 & $\mathrm{U}$ \\
\hline $108-88-3$ & Toluene & 0.50 & $\mathrm{U}$ \\
\hline $10061-02-6$ & trans-1,3-Dichloropropene & 0.50 & $\mathrm{U}$ \\
\hline $79-00-5$ & $1,1,2$-Trichloroethane & 0.50 & $\mathrm{U}$ \\
\hline $127-18-4$ & Tetrachloroethene & 0.50 & $\mathrm{U}$ \\
\hline $591-78-6$ & 2-Hexanone & 5.0 & $\bar{U}$ \\
\hline $124-48-1$ & Dibromochloromethane & 0.50 & $\mathrm{U}$ \\
\hline $106-93-4$ & 1,2-Dibromoethane & 0.50 & $\mathrm{U}$ \\
\hline $108-90-7$ & Chlorobenzene & 0.50 & $\mathrm{U}$ \\
\hline $100-41-4$ & Ethylbenzene & 0.50 & $\bar{U}$ \\
\hline $95-47-6$ & o-Xylene & 0.50 & $\mathrm{U}$ \\
\hline $179601-23-1$ & $\mathrm{~m}, \mathrm{p}$-Xylene & 0.50 & $\mathrm{U}$ \\
\hline $100-42-5$ & styrene & 0.50 & $\mathrm{U}$ \\
\hline $75-25-2$ & Bromoform & 0.50 & $\mathrm{U}$ \\
\hline $98-82-8$ & Isopropylbenzene & 0.50 & $\mathrm{U}$ \\
\hline $79-34-5$ & $1,1,2,2$-Tetrachloroethane & 0.50 & $\mathrm{U}$ \\
\hline $541-73-1$ & 1,3-Dichlorobenzene & 0.50 & $\mathrm{U}$ \\
\hline $106-46-7$ & 1,4-Dichlorobenzene & 0.50 & $\mathrm{U}$ \\
\hline $95-50-1$ & 1,2-Dichlorobenzene & 0.50 & $\mathrm{U}$ \\
\hline $96-12-8$ & 1,2-Dibromo-3-Chloropropane & 0.50 & $\mathrm{U}$ \\
\hline $120-82-1$ & $1,2,4$-Trichlorobenzene & 0.50 & $\mathrm{U}$ \\
\hline $87-61-6$ & $1,2,3$-Trichlorobenzene & 0.066 & $\mathrm{~J}$ \\
\hline
\end{tabular}

Lab Sample ID: MB 200-7052/4

Lab File ID: JBMD04.D

Date Received:

Date Analyzed: 09/23/2010

Dilution Factor: 1.0

Soil Aliquot Volume: 
Lab Name: TESTAMERICA BURLINGTON

Contract: $8 \mathrm{E}-00302$

Lab Code: STLV Case No.: CENTRA Mod. Ref No.: SDG No.: 200-1629

Matrix: (SOIL/SED/WATER)

Water

Sample wt/vol: 25.0 $(\mathrm{g} / \mathrm{mL}) \mathrm{mL}$

Level: (TRACE or LOW/MED) TRACE

\% Moisture: not dec.

GC Column: $\mathrm{DB}-624$ ID: $0.20 \quad(\mathrm{~mm})$

Soil Extract Volume: (uL) CONCENTRATION UNITS: (ug/L or $u g / \mathrm{kg}) \mathrm{ug} / \mathrm{L}$
Lab Sample ID: MB 200-7052/4

Lab File ID: JBMD04.D

Date Received:

Date Analyzed: 09/23/2010

Dilution Factor: 1.0 Soil Aliquot Volume: (uL)

Purge Volume: 25.0 $(\mathrm{mL})$

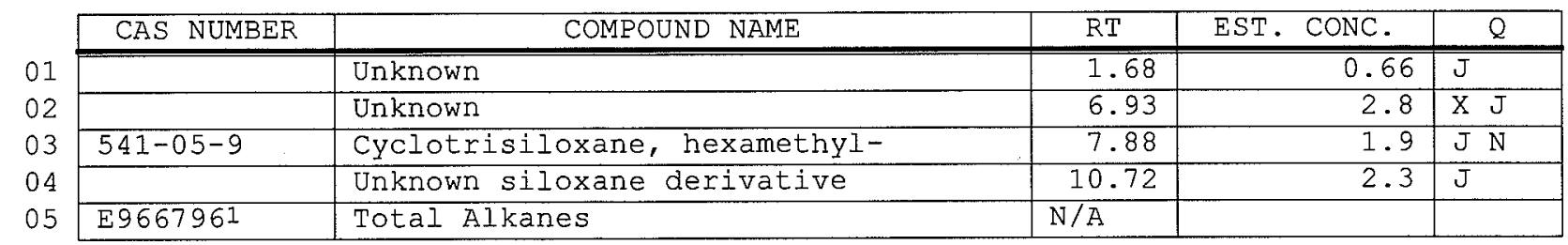

1 EPA-designated Registry Number. 
Lab Name: TESTAMERICA BURLINGTON

Contract: $8 \mathrm{E}-00302$

Lab Code: STLV Case No.: CENTRA Mod. Ref No.: SDG No.: 200-1629

Matrix: (SOIL/SED/WATER) Water

Sample wt/vol: $25.0 \quad(\mathrm{~g} / \mathrm{mL}) \mathrm{mL}$

Level: (TRACE/LOW/MED) TRACE

\% Moisture: not dec.

GC Column: DB-624 ID: $0.20 \quad(\mathrm{~mm})$

Soil Extract Volume: (uL) (uL) Purge Volume: 25.0 (mL)
Lab Sample ID: MB 200-7.048/4

Lab File ID: JBME04.D

Date Received:

Date Analyzed: 09/24/2010

Dilution Factor: 1.0

Soil Aliquot Volume: (uL)

\begin{tabular}{|c|c|c|c|}
\hline CAS NO. & COMPOUND & $\begin{array}{l}\text { CONCENTRATION UNITS: } \\
(\mathrm{ug} / \mathrm{L} \text { or } \mathrm{ug} / \mathrm{kg}) \mathrm{ug} / \mathrm{L}\end{array}$ & Q \\
\hline $75-71-8$ & Dichlorodifluoromethane & 0.50 & $\overline{\mathrm{U}}$ \\
\hline $74-87-3$ & Chloromethane & 0.50 & $\mathrm{U}$ \\
\hline $75-01-4$ & Vinyl chloride & 0.50 & $\mathrm{U}$ \\
\hline $74-83-9$ & Bromomethane & 0.50 & $\mathrm{U}$ \\
\hline $75-00-3$ & Chloroethane & 0.50 & $\mathrm{U}$ \\
\hline $75-69-4$ & Trichlorofluoromethane & 0.50 & $\mathrm{U}$ \\
\hline $75-35-4$ & 1,1-Dichloroethene & 0.50 & $\mathrm{U}$ \\
\hline $76-13-1$ & 1,1,2-Trichloro-1,2,2-trifluoroethane & 0.50 & $\mathrm{U}$ \\
\hline $67-64-1$ & Acetone & 3.7 & $\mathrm{~J}$ \\
\hline $75-15-0$ & Carbon disulfide & 0.50 & $\mathrm{U}$ \\
\hline $79-20-9$ & Methyl acetate & 0.50 & $\mathrm{U}$ \\
\hline $75-09-2$ & Methylene Chloride & 0.50 & $\mathrm{U}$ \\
\hline $156-60-5$ & trans-1,2-Dichloroethene & 0.50 & $\mathrm{U}$ \\
\hline $1634-04-4$ & Methyl tert-butyl ether & 0.50 & $\mathrm{U}$ \\
\hline $75-34-3$ & 1,1-Dichloroethane & 0.50 & $\mathrm{U}$ \\
\hline $156-59-2$ & cis-1,2-Dichloroethene & 0.50 & $\mathrm{U}$ \\
\hline $78-93-3$ & 2-Butanone & 5.0 & $\mathrm{U}$ \\
\hline $74-97-5$ & Bromochloromethane & 0.50 & $\mathrm{U}$ \\
\hline $67-66-3$ & Chloroform & 0.50 & $\mathrm{U}$ \\
\hline $71-55-6$ & 1,1,1-Trichloroethane & 0.50 & $\mathrm{U}$ \\
\hline $110-82-7$ & Cyclohexane & 0.50 & $\mathrm{U}$ \\
\hline $56-23-5$ & Carbon tetrachloride & 0.50 & $\mathrm{U}$ \\
\hline $71-43-2$ & Benzene & 0.50 & $\mathrm{U}$ \\
\hline $107-06-2$ & 1,2-Dichloroethane & 0.50 & $\mathrm{U}$ \\
\hline
\end{tabular}

Report 1,4-Dioxane for Low-Medium VOA analysis only 
Lab Name: TESTAMERICA BURLINGTON

Contract: $8 \mathrm{E}-00302$

Lab Code: STLV

Case No.: CENTRA Mod. Ref No.:

SDG NO.: 200-1629

Matrix: (SOIL/SED/WATER) Water

Sample wt/vol: 25.0 (g/mL) $\mathrm{mL}$

Level: (TRACE/LOW/MED) TRACE

\% Moisture: not dec.

GC Column: DB-624

ID: $0.20 \quad(\mathrm{~mm})$

Soil Extract Volume:

(uI)

(mL)
Lab Sample ID: MB 200-7048/4

Lab File ID: JBME04.D

Date Received:

Date Analyzed: 09/24/2010

Dilution Factor: 1.0

Soil Aliquot volume:

(uL)

\begin{tabular}{|c|c|c|c|}
\hline CAS NO. & COMPOUND & $\begin{array}{l}\text { CONCENTRATION UNITS: } \\
(u g / L \text { or } u g / k g) u g / L\end{array}$ & $Q$ \\
\hline $79-01-6$ & Trichloroethene & 0.50 & $\overline{\mathrm{U}}$ \\
\hline $108-87-2$ & Methylcyclohexane & 0.50 & $\mathrm{U}$ \\
\hline $78-87-5$ & 1,2-Dichloropropane & 0.50 & $\mathrm{U}$ \\
\hline $75-27-4$ & Bromodichloromethane & 0.50 & $\mathrm{U}$ \\
\hline $10061-01-5$ & cis-1,3-Dich loropropene & 0.50 & $\mathrm{U}$ \\
\hline $108-10-1$ & 4-Methyl-2-pentanone & 5.0 & $\mathrm{U}$ \\
\hline $108-88-3$ & Toluene & 0.50 & $\mathrm{U}$ \\
\hline $10061-02-6$ & trans-1,3-Dichloropropene & 0.50 & $\bar{U}$ \\
\hline $79-00-5$ & $1,1,2-\operatorname{Trichloroethane}$ & 0.50 & $\mathrm{U}$ \\
\hline $127-18-4$ & Tetrachloroethene & 0.50 & $\mathrm{U}$ \\
\hline $591-78-6$ & 2-Hexanone & 5.0 & $\mathrm{U}$ \\
\hline $124-48-1$ & Dibromochloromethane & 0.50 & $\overline{\mathrm{U}}$ \\
\hline $106-93-4$ & 1,2-Dibromoethane & 0.50 & $\mathrm{U}$ \\
\hline $108-90-7$ & Chlorobenzene & 0.50 & $\bar{U}$ \\
\hline $100-41-4$ & Ethylbenzene & 0.50 & $\mathrm{U}$ \\
\hline $95-47-6$ & o-xylene & 0.50 & $\mathrm{U}$ \\
\hline $179601-23-1$ & m, p-xylene & 0.50 & $\bar{U}$ \\
\hline $100-42-5$ & Styrene & 0.50 & $\bar{U}$ \\
\hline $75-25-2$ & Bromoform & 0.50 & $\mathrm{U}$ \\
\hline $98-82-8$ & Isopropylbenzene & 0.50 & $\mathrm{U}$ \\
\hline $79-34-5$ & $1,1,2,2$-Tetrachloroethane & 0.50 & $\mathrm{U}$ \\
\hline $541-73-1$ & 1,3-Dichlorobenzene & 0.50 & $\mathrm{U}$ \\
\hline $106-46-7$ & 1,4-Dichlorobenzene & 0.50 & $\mathrm{U}$ \\
\hline $95-50-1$ & 1,2-Dichlorobenzene & 0.50 & $\mathrm{U}$ \\
\hline $96-12-8$ & 1,2-Dibromo-3-Chloropropane & 0.50 & $\overline{\mathrm{U}}$ \\
\hline $120-82-1$ & $1,2,4$-Trichlorobenzene & 0.50 & $\mathrm{U}$ \\
\hline $87-61-6$ & $1,2,3$-Trichlorobenzene & 0.084 & $\mathrm{~J}$ \\
\hline
\end{tabular}


IJ - FORM I VOA-TIC

VOLATILE ORGANICS ANALYSIS DATA SHEET

TENTATIVELY IDENTIFIED COMPOUNDS
EPA SAMPLE NO.

VBLKJG

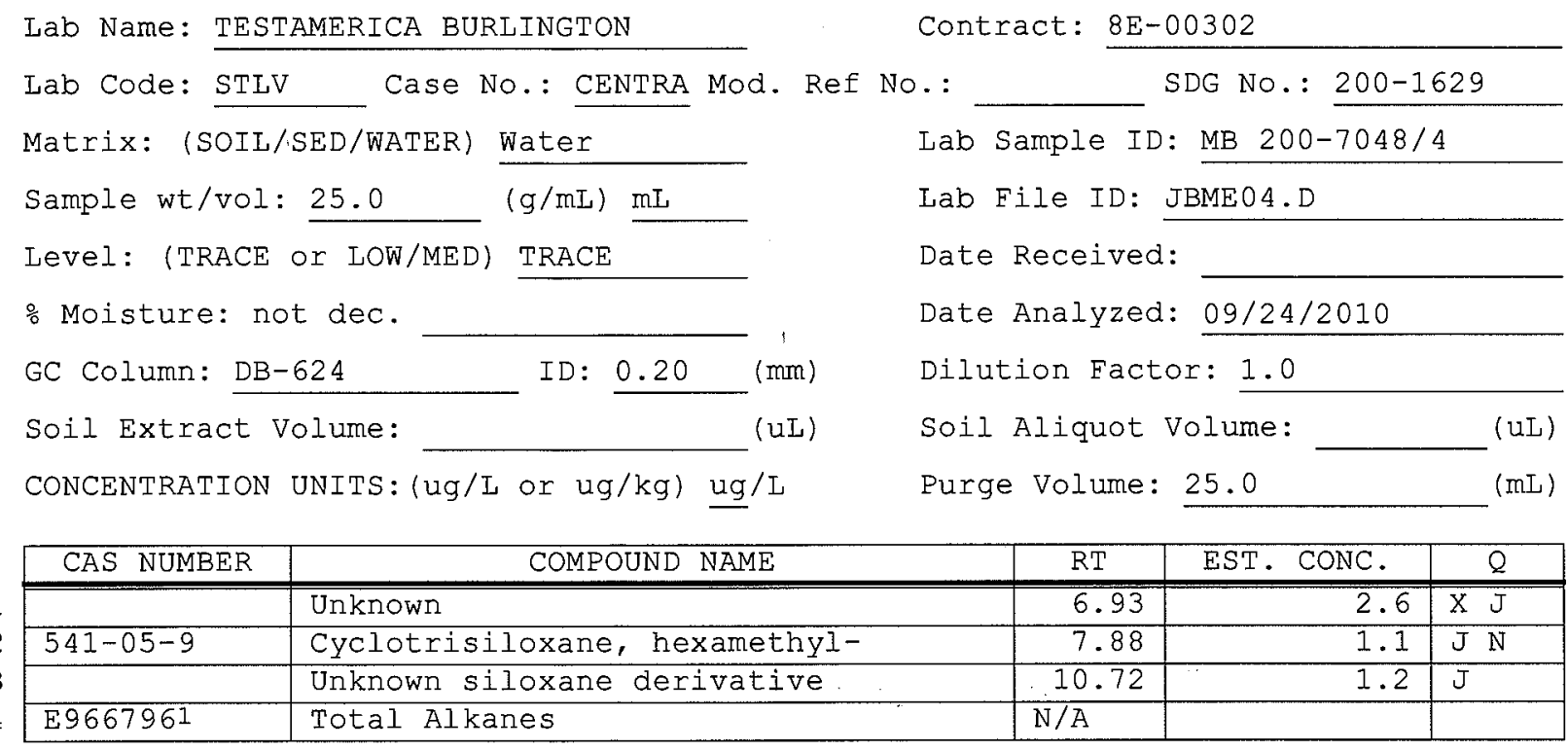

lEPA-designated Registry Number. 
Lab Name: TESTAMERICA BURLINGTON

Lab Code: STLV

Matrix: (SOIL/SED/WATER)

Sample wt/vol: 25.0

Case No.: CENTRA Mod. Ref No.:

Contract: $8 \mathrm{E}-00302$

Level: (TRACE/LOW/MED) TRACE

\% Moisture: not dec.

GC Column: DB-624

ID $: 0.20$ $(\mathrm{mm})$

Soil Extract Volume:
Water

$(\mathrm{g} / \mathrm{mL}) \mathrm{mL}$
Lab Sample ID: 200-1629-6

Lab File ID: JBME06.D

Date Received:

Date Analyzed: 09/24/2010

Dilution Factor: 1.0

Soil Aliquot Volume:

(uL)
(mL)

\begin{tabular}{|l|l|c|c|}
\hline CAS NO. & Compound & $\begin{array}{c}\text { CONCENTRATION UNITS: } \\
\text { (ug/L or ug/kg) ug/L }\end{array}$ & Q \\
\hline \hline $75-71-8$ & Dichlorodifluoromethane & 0.50 & $\mathrm{U}$ \\
\hline $74-87-3$ & Chloromethane & 0.50 & $\mathrm{U}$ \\
\hline $75-01-4$ & Vinyl chloride & 0.50 & $\mathrm{U}$ \\
\hline $74-83-9$ & Bromomethane & 0.50 & $\mathrm{U}$ \\
\hline $75-00-3$ & Chloroethane & 0.50 & $\mathrm{U}$ \\
\hline $75-69-4$ & Trichlorofluoromethane & 0.50 & $\mathrm{U}$ \\
\hline $75-35-4$ & $1,1-$ Dichloroethene & 0.50 & $\mathrm{U}$ \\
\hline $76-13-1$ & 1,1,2-Trichloro-1,2,2-trifluoroethane & 0.50 & $\mathrm{U}$ \\
\hline $67-64-1$ & Acetone & 1.8 & $\mathrm{~J}$ \\
\hline $75-15-0$ & Carbon disulfide & 0.50 & $\mathrm{U}$ \\
\hline $79-20-9$ & Methyl acetate & 0.50 & $\mathrm{U}$ \\
\hline $75-09-2$ & Methylene Chloride & 0.50 & $\mathrm{U}$ \\
\hline $156-60-5$ & trans-1,2-Dichloroethene & 0.50 & $\mathrm{U}$ \\
\hline $1634-04-4$ & Methyl tert-butyl ether & 0.50 & $\mathrm{U}$ \\
\hline $75-34-3$ & $1,1-$ Dichloroethane & 0.50 & $\mathrm{U}$ \\
\hline $156-59-2$ & Cis-1,2-Dichloroethene & 0.50 & $\mathrm{U}$ \\
\hline $78-93-3$ & 2-Butanone & 5.0 & $\mathrm{U}$ \\
\hline $74-97-5$ & Bromochloromethane & 0.50 & $\mathrm{U}$ \\
\hline $67-66-3$ & Chloroform & 0.50 & $\mathrm{U}$ \\
\hline $71-55-6$ & $1,1,1-T r i c h l o r o e t h a n e$ & 0.50 & $\mathrm{U}$ \\
\hline $110-82-7$ & Cyclohexane & 0.50 & $\mathrm{U}$ \\
\hline $56-23-5$ & Carbon tetrachloride & 0.50 & $\mathrm{U}$ \\
\hline $71-43-2$ & Benzene & 0.50 & $\mathrm{U}$ \\
\hline $107-06-2$ & $1,2-$ Dichloroethane & 0.50 & $\mathrm{U}$ \\
\hline
\end{tabular}

Report 1,4-Dioxane for Low-Medium VOA analysis only 
Lab Name: TESTAMERICA BURLINGTON

Contract: $8 \mathrm{E}-00302$

Lab Code: STLV

Case No.: CENTRA Mod. Ref No.:

SDG No.: 200-1629

Matrix: (SOIL/SED/WATER)

Sample wt/vol: 25.0

Water

$(\mathrm{g} / \mathrm{mL}) \mathrm{mL}$

Level: (TRACE/LOW/MED) TRACE

음 Moisture: not dec.

GC Column: DB-624

ID: 0.20 (mm)

Soil Extract Volume: (uL)

Purge Volume: 25.0
Lab Sample ID: 200-1629-6

Lab File ID: JBME06.D

Date Received:

Date Analyzed: 09/24/2010

Dilution Factor: 1.0

Soil Aliquot Volume:

(uL)

\begin{tabular}{|c|c|c|c|}
\hline CAS NO. & COMPOUND & $\begin{array}{l}\text { CONCENTRATION UNITS: } \\
(\mathrm{ug} / \mathrm{L} \text { or } \mathrm{ug} / \mathrm{kg}) \mathrm{ug} / \mathrm{L}\end{array}$ & $Q$ \\
\hline $79-01-6$ & Trichloroethene & 0.50 & $\overline{\mathrm{U}}$ \\
\hline $108-87-2$ & Methylcyclohexane & 0.50 & $\overline{\mathrm{U}}$ \\
\hline $78-87-5$ & 1,2-Dichloropropane & 0.50 & $\overline{\mathrm{U}}$ \\
\hline $75-27-4$ & Bromodichloromethane & 0.50 & $\overline{\mathrm{U}}$ \\
\hline $10061-01-5$ & cis-1,3-Dichloropropene & 0.50 & $\mathrm{U}$ \\
\hline $108-10-1$ & 4-Methyl-2-pentanone & 5.0 & $\bar{U}$ \\
\hline $108-88-3$ & Toluene & 0.50 & $\overline{\mathrm{U}}$ \\
\hline $10061-02-6$ & trans-1,3-Dichloropropene & 0.50 & $\overline{\mathrm{U}}$ \\
\hline $79-00-5$ & $1,1,2$-Trichloroethane & 0.50 & $\overline{\mathrm{U}}$ \\
\hline $127-18-4$ & Tetrachloroethene & 0.50 & $\mathrm{U}$ \\
\hline $591-78-6$ & 2-Hexanone & 5.0 & $\mathrm{U}$ \\
\hline $124-48-1$ & Dibromochloromethane & 0.50 & $\mathrm{U}$ \\
\hline $106-93-4$ & 1,2-Dibromoethane & 0.50 & $\bar{U}$ \\
\hline $108-90-7$ & Chlorobenzene & 0.50 & $\overline{\mathrm{U}}$ \\
\hline $100-41-4$ & Ethylbenzene & 0.50 & $\mathrm{U}$ \\
\hline $95-47-6$ & o-xylene & 0.50 & $\mathrm{U}$ \\
\hline $179601-23-1$ & $\mathrm{~m}, \mathrm{p}-\mathrm{xy}$ lene & 0.50 & $\mathrm{U}$ \\
\hline $100-42-5$ & styrene & 0.50 & $\mathrm{U}$ \\
\hline $75-25-2$ & Bromoform & 0.50 & $\mathrm{U}$ \\
\hline $98-82-8$ & Isopropylbenzene & 0.50 & $\mathrm{U}$ \\
\hline $79-34-5$ & $1,1,2,2-$ Tetrachloroethane & 0.50 & $\mathrm{U}$ \\
\hline $541-73-1$ & 1,3-Dichlorobenzene & 0.50 & $\mathrm{U}$ \\
\hline $106-46-7$ & 1,4-Dichlorobenzene & 0.50 & $\mathrm{U}$ \\
\hline $95-50-1$ & 1,2-Dichlorobenzene & 0.50 & $\mathrm{U}$ \\
\hline $96-12-8$ & 1,2-Dibromo-3-Chloropropane & 0.50 & $\mathrm{U}$ \\
\hline $120-82-1$ & $1,2,4$-Trichlorobenzene & 0.50 & $\overline{\mathrm{U}}$ \\
\hline $87-61-6$ & 1,2,3-Trichlorobenzene & 0.50 & $\mathrm{U}$ \\
\hline
\end{tabular}


$1 \mathrm{~J}-$ FORM I VOA-TIC

VOLATILE ORGANICS ANALYSIS DATA SHEET

TENTATIVELY IDENTIFIED COMPOUNDS
EPA SAMPLE NO.

VHBLKO1
Lab Name: TESTAMERICA BURLINGTON

Lab Code: STLV

Matrix: (SOIL/SED/WATER)

Sample wt/vol: 25.0

water

$(\mathrm{g} / \mathrm{mL}) \mathrm{mL}$

Level: (TRACE or LOW/MED) TRACE

응 Moisture: not dec.

GC Column: DB-624

ID: 0.20 (mm)

Soil Extract Volume:

CONCENTRATION UNITS: (ug/L or ug/kg) $\mathrm{ug} / \mathrm{L}$
Contract: $8 \mathrm{E}-00302$

SDG No.: 200-1629

Lab Sample ID: 200-1629-6

Lab File ID: JBME06.D

Date Received:

Date Analyzed: 09/24/2010

Dilution Factor: 1.0

Soil Aliquot volume:

Purge Volume: 25.0 (mL)

\begin{tabular}{|c|c|c|c|c|c|}
\hline & CAS NUMBER & COMPOUND NAME & RT & EST. CONC. & $Q$ \\
\hline 01 & & Unknown & 6.93 & 2.7 & $\overline{B \times \mathrm{J}}$ \\
\hline 02 & $541-05-9$ & Cyclotrisiloxane, hexamethyl- & 7.88 & 1.4 & $\mathrm{~B} \mathrm{~J} \mathrm{~N}$ \\
\hline 03 & & Unknown siloxane derivative & 10.72 & 1.2 & $\mathrm{~B} \mathrm{~J}$ \\
\hline 04 & E9667961 & Total Alkanes & $\mathrm{N} / \mathrm{A}$ & & \\
\hline
\end{tabular}

1EPA-designated Registry Number. 
Argonne

\section{Environmental Science Division}

Argonne National Laboratory

9700 South Cass Avenue, Bldg. 203

Argonne, IL 60439-4843

www.anl.gov 